\title{
Asymmetric Intramolecular Rauhut-Currier Reaction and Its \\ Desymmetric Version via Double Thiol/Phase-Transfer Catalysis
}

\author{
Ying Jiang,$^{\dagger}$ Yang Yang, ${ }^{\dagger}$ Qing He,${ }^{\dagger}$ Wei Du, ${ }^{* \dagger}$ and Ying-Chun Chen $*^{\dagger}, \dagger$
}

${ }^{\dagger}$ Key Laboratory of Drug-Targeting and Drug Delivery System of the Ministry of Education and Sichuan

Research Center for Drug Precision Industrial Technology, West China School of Pharmacy, Sichuan University, Chengdu 610041, China

${ }^{\ddagger}$ College of Pharmacy, Third Military Medical University, Shapingba, Chongqing 400038, China

Email: duweiyb@scu.edu.cn; ycchen@scu.edu.cn

\section{Supporting Information}

\section{Table of Contents}

1. More screening conditions for intramolecular RC reaction of bis(enones) 1a ..................................... S2

2. More screening conditions for intramolecular RC reaction of bis(enones) 3a....................................S3

3. General procedure for intramolecular RC reaction of bis(enones) 1 ................................................... S6

4. General procedure for intramolecular RC reaction of bis(enones) 3 ............................................... S9

5. Screening conditions for desymmetric intramolecular RC reaction of bis(enones) 5a ...................... S11

6. General procedure for desymmetric intramolecular $\mathrm{RC}$ reaction of bis(enones) 5 ........................... S12

7. More exploration on intramolecular RC reaction of diversely structured substrates ........................ S16

8. Transformations of products $2 \mathrm{c}$ and $6 \mathrm{~m}$......................................................................................................... S19

9. Crystal data and structural refinement for enantiopure 8 and 15 (derivative of $6 \mathrm{~m}$ ) ....................... S19

10. Proposed transition state for the desymmetric RC reaction..................................................................... S23

11. NMR, HRMS spectra and HPLC chromatograms.................................................................................. S24 


\section{More screening conditions for intramolecular $\mathrm{RC}$ reaction of bis(enones) 1}

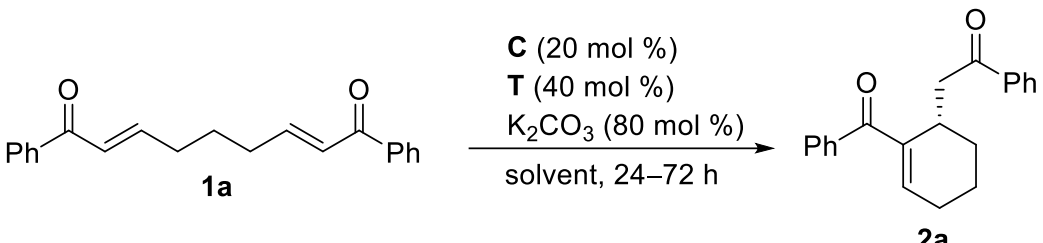

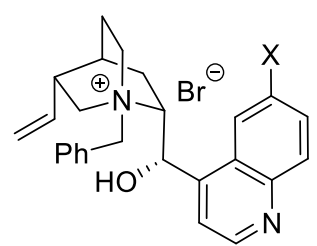

C1 $\mathrm{X}=\mathrm{H}$

C2 $\mathrm{X}=\mathrm{OCH}_{3}$<smiles>O=C(O)c1ccccc1S</smiles>

T1

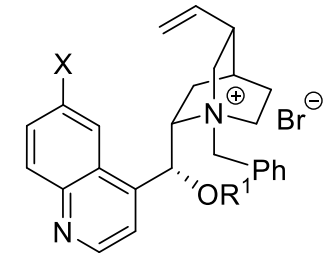

C3 $\mathrm{X}=\mathrm{OCH}_{3}, \mathrm{R}^{1}=\mathrm{H}$

$$
\text { C10 X }=\mathrm{H}, \mathrm{R}^{1}=\mathrm{H}
$$$$
\text { C11 } X=H, R^{1}=B n
$$<smiles>Oc1ccccc1S</smiles>

T2

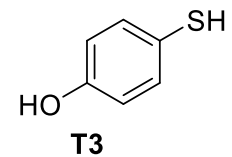

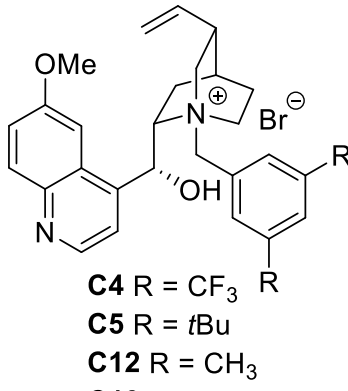

$\mathrm{C} 13 \mathrm{R}=\mathrm{Ph}$
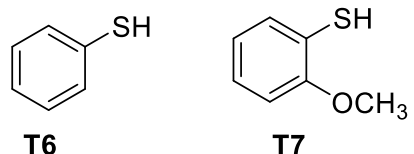

\begin{tabular}{|c|c|c|c|c|c|c|c|}
\hline entry $^{a}$ & $\mathbf{C}$ & $\mathbf{T}$ & solvent & temperature $\left({ }^{\circ} \mathrm{C}\right)$ & $t(\mathrm{~h})$ & yield $(\%)^{b}$ & ee $(\%)^{c}$ \\
\hline 1 & C1 & $\mathbf{T 1}$ & toluene & 50 & 72 & 57 & -9 \\
\hline 2 & C1 & $\mathbf{T} 2$ & toluene & 50 & 24 & 79 & -32 \\
\hline 3 & C1 & T3 & toluene & 50 & 72 & NR & - \\
\hline 4 & C1 & T6 & toluene & 50 & 72 & NR & - \\
\hline 5 & C1 & T7 & toluene & 50 & 72 & 63 & -21 \\
\hline 6 & C1 & - & toluene & 50 & 72 & 39 & -6 \\
\hline 7 & $\mathrm{C} 2$ & $\mathbf{T} 2$ & toluene & 50 & 24 & 83 & -59 \\
\hline 8 & C3 & $\mathbf{T} 2$ & toluene & 50 & 24 & 83 & 77 \\
\hline 9 & C10 & $\mathbf{T} 2$ & toluene & 50 & 24 & 87 & 48 \\
\hline 10 & C11 & $\mathbf{T} 2$ & toluene & 50 & 24 & NR & - \\
\hline 11 & C4 & $\mathbf{T} 2$ & toluene & 50 & 24 & 78 & 90 \\
\hline 12 & C5 & $\mathbf{T} 2$ & toluene & 50 & 24 & 86 & 89 \\
\hline 13 & $\mathrm{C} 12$ & $\mathbf{T} 2$ & toluene & 50 & 24 & 81 & 74 \\
\hline 14 & C13 & $\mathbf{T} 2$ & toluene & 50 & 24 & 85 & 85 \\
\hline 15 & C4 & $\mathbf{T} 2$ & toluene & $\mathrm{rt}$ & 48 & 86 & 92 \\
\hline 16 & C4 & $\mathbf{T} 2$ & toluene & 4 & 72 & 66 & 96 \\
\hline
\end{tabular}




$\begin{array}{llllllll}17 & \mathbf{C 5} & \mathbf{T 2} & \text { toluene } & \mathrm{rt} & 48 & 91 & 91 \\ 18 & \mathbf{C 5} & \mathbf{T 2} & \text { toluene } & 4 & 72 & 53 & 97 \\ 19 & \mathbf{C 5} & \mathbf{T 2} & \mathrm{THF} & \mathrm{rt} & 48 & 73 & 61 \\ 20 & \mathbf{C 5} & \mathbf{T 2} & \text { EtOAc } & \mathrm{rt} & 48 & 95 & 83 \\ 21 & \mathbf{C 5} & \mathbf{T 2} & \text { acetone } & \mathrm{rt} & 48 & 86 & 54 \\ 22 & \mathbf{C 5} & \mathbf{T 2} & \mathrm{CHCl}_{3} & \mathrm{rt} & 48 & 89 & 84 \\ 23^{d} & \mathbf{C 5} & \mathbf{T 2} & \text { toluene } & \mathrm{rt} & 72 & 60 & 93 \\ 24^{e} & \mathbf{C 5} & \mathbf{T 2} & \text { toluene } & \mathrm{rt} & 48 & 91 & 87 \\ \mathbf{2 5} & \mathbf{C 5} & \mathbf{T 2} & \text { toluene } & \mathbf{r t} & \mathbf{4 8} & \mathbf{9 2} & \mathbf{9 1} \\ 26^{g} & \mathbf{C 5} & \mathbf{T 2} & \text { toluene } & \mathrm{rt} & 48 & 80 & 89\end{array}$

${ }^{a}$ Unless noted otherwise, reactions were performed with $\mathbf{1 a}(0.05 \mathrm{mmol})$, phase transfer catalyst $\mathbf{C}(20 \mathrm{~mol} \%)$, thiol T $(40 \mathrm{~mol} \%)$ and $\mathrm{K}_{2} \mathrm{CO}_{3}(80 \mathrm{~mol} \%)$ in solvent $(1 \mathrm{~mL}) .{ }^{b}$ Yield of the isolated product. ${ }^{c}$ Determined by HPLC analysis on a chiral stationary phase. ${ }^{d}$ With $\mathbf{C 5}(10 \mathrm{~mol} \%)$. ${ }^{e}$ With $\mathbf{T 2}(20 \mathrm{~mol} \%) .{ }^{f}$ With $\mathrm{K}_{2} \mathrm{CO}_{3}(60 \mathrm{~mol} \%) .{ }^{g}$ With $\mathrm{K}_{2} \mathrm{CO}_{3}(40 \mathrm{~mol} \%)$. NR = No reaction.

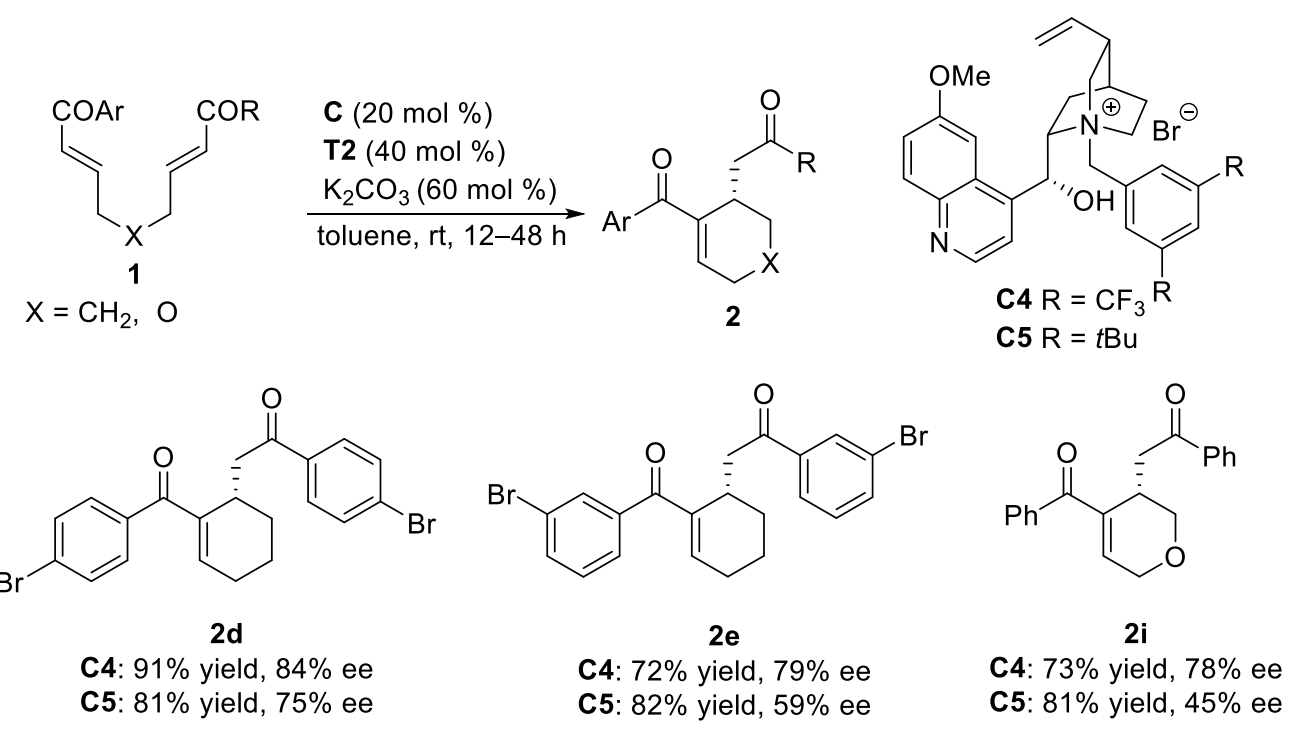

\section{More screening conditions for intramolecular $\mathrm{RC}$ reaction of bis(enones) $3 \mathrm{a}$}

PTC C5 with 3,5-di-tert-butyl substitutions was shown to be a highly effective catalyst for the asymmetric intramolecular RC reaction affording six-membered ring products, but it afforded inferior results for the more challenging bis(enones) 3a (83\% yield, 36\% ee) with T2. So different modified PTCs were tested, but the results were not satisfying. 


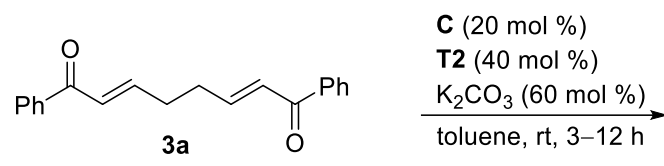<smiles>O=C(C[C]1CCC=C1C(=O)c1ccccc1)c1ccccc1</smiles>

4a

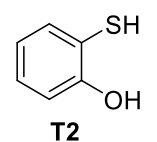

T2

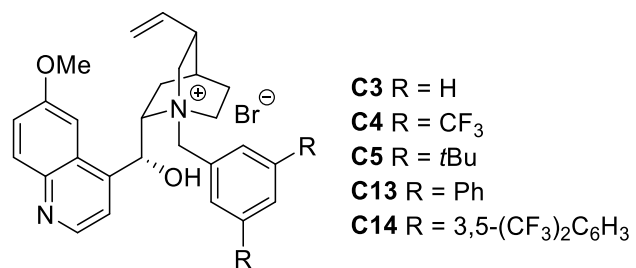

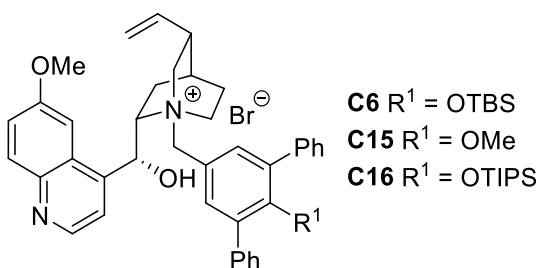

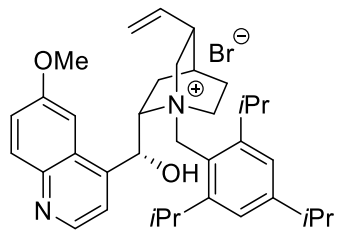

C17
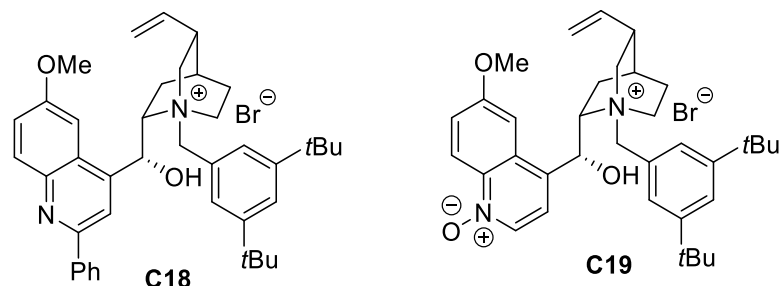

\begin{tabular}{|c|c|c|c|c|}
\hline entry $^{a}$ & $\mathbf{C}$ & $t(\mathrm{~h})$ & yield $(\%)^{b}$ & ee $(\%)^{c}$ \\
\hline 1 & C3 & 3 & 80 & 16 \\
\hline 2 & C4 & 3 & 72 & 32 \\
\hline 3 & C5 & 3 & 83 & 36 \\
\hline 4 & C13 & 3 & 80 & 41 \\
\hline 5 & C14 & 3 & 78 & 32 \\
\hline 6 & C6 & 3 & 85 & 56 \\
\hline 7 & C15 & 3 & 81 & 48 \\
\hline $8^{d}$ & C16 & 3 & 78 & 45 \\
\hline 9 & C17 & 12 & 69 & 9 \\
\hline 10 & C18 & 6 & 75 & 11 \\
\hline 11 & C19 & 6 & 70 & 26 \\
\hline
\end{tabular}

${ }^{a}$ Unless noted otherwise, reactions were performed with 3a $(0.05 \mathrm{mmol})$, phase transfer catalyst $\mathbf{C}(20 \mathrm{~mol} \%)$, thiol $\mathbf{T}(40 \mathrm{~mol} \%)$ and $\mathrm{K}_{2} \mathrm{CO}_{3}(60 \mathrm{~mol} \%)$ in toluene $(1 \mathrm{~mL})$ at rt. ${ }^{b}$ Yield of the isolated product. ${ }^{c}$ Determined by HPLC analysis on a chiral stationary phase.

In addition, a few thiols were tested with the combination of $\mathbf{C 6}$, but the results were not satisfying too. 
<smiles>O=C(/C=C/CC/C=C/C(=O)c1ccccc1)c1ccccc1</smiles>

$3 a$<smiles>O=C(O)c1ccccc1S</smiles>

$15 \%$ yield, $5 \%$ ee<smiles>FC(F)(F)Nc1ccccc1S</smiles>

T10
C6 $(20 \mathrm{~mol} \%)$

T $(40 \mathrm{~mol} \%)$

$\underset{\text { toluene, } 50^{\circ} \mathrm{C}, 24 \mathrm{~h}}{\stackrel{\mathrm{K}_{2} \mathrm{CO}_{3}(60 \mathrm{~mol} \%)}{\longrightarrow}}$

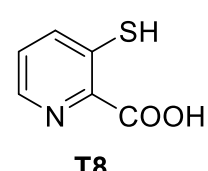

$13 \%$ yield, $-14 \%$ ee<smiles>O=[N+]([O-])c1ccccc1S</smiles>

T11<smiles>O=C(C[C@H]1CCC=C1C(=O)c1ccccc1)c1ccccc1</smiles>

4a

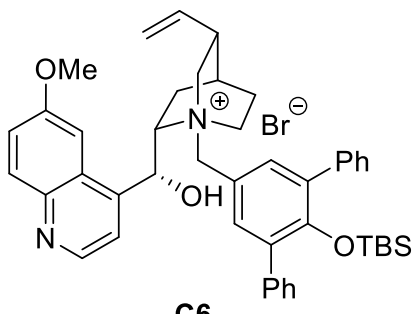

C6

$60 \%$ yield, $-4 \%$ ee

$65 \%$ yield, $20 \%$ ee

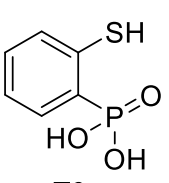

T9

$16 \%$ yield, $2 \%$ ee<smiles>Sc1ccccc1S</smiles>

T12

Some chiral binol-derived thiols were tried as well, and it was pleasing that these chiral thiols could afford the RC product smoothly in combination with a matched chiral phase transfer catalyst. Product 4a could be obtained in good data when T4 and $\mathbf{C 6}$ were employed.

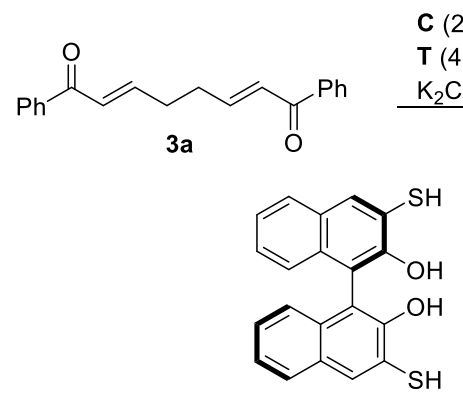

T4
C $(20 \mathrm{~mol} \%)$ T $(40 \mathrm{~mol} \%)$ $\mathrm{K}_{2} \mathrm{CO}_{3}(60 \mathrm{~mol} \%)$<smiles>Oc1cc2ccccc2cc1S</smiles><smiles>Cc1c(O)c(S)cc2ccccc12</smiles>

T13<smiles>O=C(CC1CCC=C1C(=O)c1ccccc1)c1ccccc1</smiles>

4a
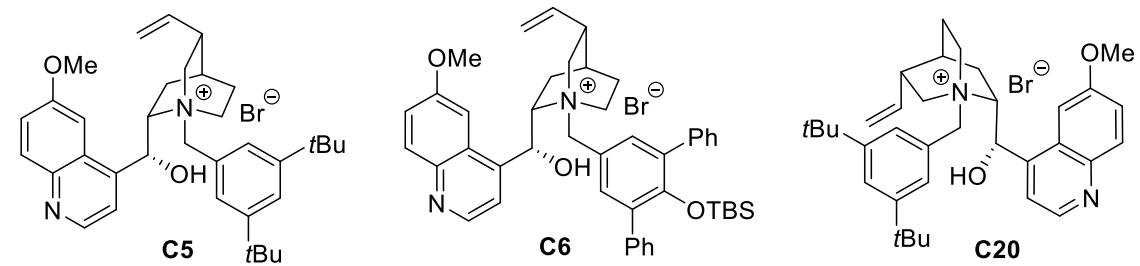

\begin{tabular}{|c|c|c|c|c|c|c|}
\hline entry $^{a}$ & $\mathbf{C}$ & $\mathbf{T}$ & temperature $\left({ }^{\circ} \mathrm{C}\right)$ & $t(\mathrm{~h})$ & yield $(\%)^{b}$ & ee $(\%)^{c}$ \\
\hline 1 & C5 & T4 & $\mathrm{rt}$ & 20 & 68 & -81 \\
\hline 2 & $\mathrm{C20}$ & $\mathbf{T 4}$ & $\mathrm{rt}$ & 20 & 60 & -10 \\
\hline 3 & TBAB & T4 & 50 & 36 & 28 & -8 \\
\hline 4 & C5 & T13 & $\mathrm{rt}$ & 48 & 56 & 47 \\
\hline 5 & $\mathrm{C20}$ & T13 & $\mathrm{rt}$ & 20 & 59 & 64 \\
\hline 6 & TBAB & T13 & 50 & 36 & 35 & 25 \\
\hline 7 & C6 & T4 & $\mathrm{rt}$ & 12 & 92 & -91 \\
\hline
\end{tabular}




$$
\begin{array}{lllllll}
8^{d} & \text { C6 } & \text { T4 } & \text { rt } & 24 & 90 & -93
\end{array}
$$

${ }^{a}$ Unless noted otherwise, reactions were performed with 3a $(0.05 \mathrm{mmol})$, phase transfer catalysts $\mathbf{C}(20 \mathrm{~mol} \%)$, thiol $\mathbf{T}(40 \mathrm{~mol} \%)$ and $\mathrm{K}_{2} \mathrm{CO}_{3}(60 \mathrm{~mol} \%)$ in toluene $(1 \mathrm{~mL}) .{ }^{b}$ Yield of the isolated product. ${ }^{c}$ Determined by HPLC analysis on a chiral stationary phase. ${ }^{d}$ With $\mathbf{C 6}(10 \mathrm{~mol} \%)$ and thiol T4 (10 mol \%).

\section{General procedure for intramolecular $\mathrm{RC}$ reaction of bis(enones) 1}

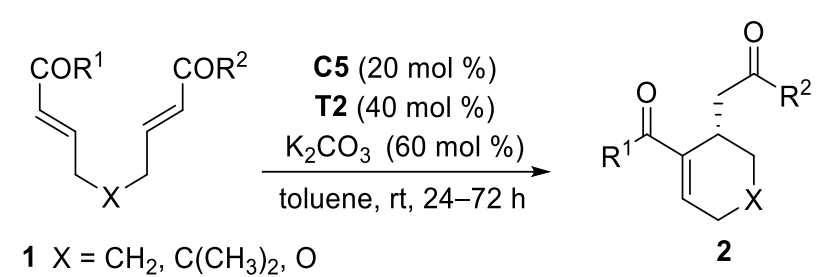

General procedure: Bis(enones) 1 (0.1 mmol), 2-mercaptophenol T2 $(0.04 \mathrm{mmol})$, catalyst $\mathbf{C 5}$ or C4 (0.02 mmol) and $\mathrm{K}_{2} \mathrm{CO}_{3}(0.06 \mathrm{mmol})$ were added into a vial equipped with a magnetic stir bar. Toluene $(1.0 \mathrm{~mL})$ was added. The mixture was stirred at room temperature for 24 to $72 \mathrm{~h}$. Purification by flash chromatography on silica gel (EtOAc/petroleum ether $=1: 40)$ gave the product 2 .

General procedure for racemic intramolecular $\mathbf{R C}$ reaction: Bis(enones) 1 (0.05 mmol), 2mercaptophenol T2 $(0.02 \mathrm{mmol}), \mathrm{TBAB}(0.01 \mathrm{mmol})$ and $\mathrm{K}_{2} \mathrm{CO}_{3}(0.03 \mathrm{mmol})$ were added into a vial equipped with a magnetic stir bar. Toluene $(1.0 \mathrm{~mL})$ was added. The mixture was stirred at $50{ }^{\circ} \mathrm{C}$ for 8 h. Purification by flash chromatography on silica gel (EtOAc/petroleum ether $=1: 40$ ) gave the racemic product 2 .<smiles>O=C(CC1CCCC=C1C(=O)c1ccccc1)c1ccccc1</smiles>

(S)-2-(2-Benzoylcyclohex-2-en-1-yl)-1-phenylethanone $\quad \mathbf{2 a} \quad \quad(2 E, 7 E)-1,9-$ Diphenylnona-2,7-diene-1,9-dione (30.4 mg, $0.1 \mathrm{mmol}$ ), 2-mercaptophenol T2 (5.2 mg, $0.04 \mathrm{mmol})$, catalyst $\mathbf{C 5}(12.6 \mathrm{mg}, 0.02 \mathrm{mmol})$ and $\mathrm{K}_{2} \mathrm{CO}_{3}(8.4 \mathrm{mg}, 0.06$ mmol) were stirred in toluene $(1.0 \mathrm{~mL})$ at $\mathrm{rt}$ for $48 \mathrm{~h}$. After completion, purification by flash chromatography on silica gel $($ EtOAc/petroleum ether $=1 / 40)$ gave $\mathbf{2 a}$.<smiles>Cc1ccc(C(=O)CC2CCCC=C2C(=O)c2ccc(C)cc2)cc1</smiles>

(S)-2-(2-(4-Methylbenzoyl)cyclohex-2-en-1-yl)-1-(p-tolyl) ethanone 2b: (2E,7E)-1,9-Di-p-tolylnona-2,7-diene-1,9-dione (33.2 mg, 0.1 mmol), 2-mercaptophenol T2 (5.2 mg, $0.04 \mathrm{mmol})$, catalyst C5 (12.6 $\mathrm{mg}, 0.02 \mathrm{mmol})$ and $\mathrm{K}_{2} \mathrm{CO}_{3}(8.4 \mathrm{mg}, 0.06 \mathrm{mmol})$ were stirred in toluene $(1 \mathrm{~mL})$ at $\mathrm{rt}$ for $48 \mathrm{~h}$. After 
completion, purification by flash chromatography on silica gel $($ EtOAc/petroleum ether $=1 / 40)$ gave 2 b.<smiles>COc1cccc(C(=O)CC2CCCC=C2C(=O)c2cccc(OC)c2)c1</smiles>

(S)-2-(2-(3-Methoxybenzoyl)cyclohex-2-en-1-yl)-1-(3methoxyphenyl)ethanone $\quad \mathbf{2 c}: \quad(2 E, 7 E)-1,9-\operatorname{Bis}(3-m e t h o x y$ phenyl)nona-2,7-diene-1,9-dione (36.4 mg, $0.1 \mathrm{mmol}), \quad 2-$ mercaptophenol T2 (5.2 mg, $0.04 \mathrm{mmol})$, catalyst C5 (12.6 mg, $0.02 \mathrm{mmol})$ and $\mathrm{K}_{2} \mathrm{CO}_{3}(8.4 \mathrm{mg}, 0.06 \mathrm{mmol})$ were stirred in toluene $(1 \mathrm{~mL})$ at $\mathrm{rt}$ for $48 \mathrm{~h}$. After completion, purification by flash chromatography on silica gel $($ EtOAc/petroleum ether $=1 / 20)$ gave 2c.<smiles>O=C(CC1CCCC=C1C(=O)c1ccc(Br)cc1)c1ccc(Br)cc1</smiles>

(S)-2-(2-(4-Bromobenzoyl)cyclohex-2-en-1-yl)-1-(4-bromophenyl) ethanone 2d: (2E,7E)-1,9-Bis(4-bromophenyl)nona-2,7-diene-1,9dione (46.0 mg, $0.1 \mathrm{mmol}$ ), 2-mercaptophenol T2 (5.2 mg, $0.04 \mathrm{mmol}$ ), catalyst $\mathbf{C 4}(12.6 \mathrm{mg}, 0.02 \mathrm{mmol})$ and $\mathrm{K}_{2} \mathrm{CO}_{3}(8.4 \mathrm{mg}, 0.06 \mathrm{mmol})$ were stirred in toluene $(1 \mathrm{~mL})$ at $\mathrm{rt}$ for $24 \mathrm{~h}$. After completion, purification by flash chromatography on silica gel $($ EtOAc/petroleum ether $=1 / 60)$ gave $\mathbf{2 d}$.<smiles>O=C(CC1CCCC=C1C(=O)c1cccc(Br)c1)c1cccc(Br)c1</smiles>

\section{(S)-2-(2-(3-Bromobenzoyl)cyclohex-2-en-1-yl)-1-(3-bromophenyl)} ethanone 2e: (2E,7E)-1,9-Bis(3-bromophenyl)nona-2,7-diene-1,9dione (46.0 mg, $0.1 \mathrm{mmol})$, 2-mercaptophenol T2 (5.2 mg, $0.04 \mathrm{mmol})$, catalyst $\mathbf{C 4}(12.6 \mathrm{mg}, 0.02 \mathrm{mmol})$ and $\mathrm{K}_{2} \mathrm{CO}_{3}(8.4 \mathrm{mg}, 0.06 \mathrm{mmol})$ were stirred in toluene $(1 \mathrm{~mL})$ at rt for $24 \mathrm{~h}$. After completion, purification by flash chromatography on silica gel (EtOAc/petroleum ether $=1 / 60)$ gave $\mathbf{2 e}$.

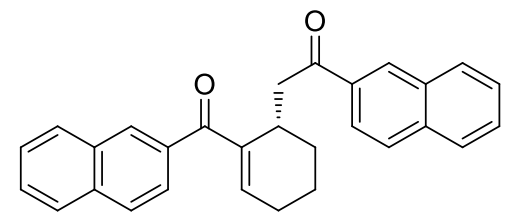

(S)-2-(2-(2-Naphthoyl)cyclohex-2-en-1-yl)-1-(naphthalen-2-yl) ethanone 2f: $(2 E, 7 E)-1,9-D i(n a p h t h a l e n-2-y l) n o n a-2,7-d i e n e-1,9-$ dione (40.4 mg, $0.1 \mathrm{mmol})$, 2-mercaptophenol T2 $(5.2 \mathrm{mg}, 0.04$ mmol), catalyst $\mathbf{C 5}(12.6 \mathrm{mg}, 0.02 \mathrm{mmol})$ and $\mathrm{K}_{2} \mathrm{CO}_{3}(8.4 \mathrm{mg}, 0.06$ mmol) were stirred in toluene $(1 \mathrm{~mL})$ at $\mathrm{rt}$ for $48 \mathrm{~h}$. After completion, purification by flash chromatography on silica gel $($ EtOAc/petroleum ether $=1 / 40)$ gave $\mathbf{2 f}$. 
<smiles>O=C(C[C@@H]1CCCC=C1C(=O)c1cccs1)c1cccs1</smiles>

(S)-1-(Thiophen-2-yl)-2-(2-(thiophene-2-carbonyl)cyclohex-2-en-1-yl)

ethanone 2g: (2E,7E)-1,9-Di(thiophen-2-yl)nona-2,7-diene-1,9-dione (31.6 mg, $0.1 \mathrm{mmol}), 2$-mercaptophenol T2 (5.2 mg, $0.04 \mathrm{mmol})$, catalyst C5 (12.6 $\mathrm{mg}, 0.02 \mathrm{mmol})$ and $\mathrm{K}_{2} \mathrm{CO}_{3}(8.4 \mathrm{mg}, 0.06 \mathrm{mmol})$ were stirred in toluene $(1 \mathrm{~mL})$ at $\mathrm{rt}$ for $48 \mathrm{~h}$. After completion, purification by flash chromatography on silica gel $($ EtOAc/petroleum ether $=1 / 40)$ gave 2g.<smiles>CC1(C)CC=C(C(=O)c2ccccc2)C(CC(=O)c2ccccc2)C1</smiles>

(S)-2-(2-Benzoyl-5,5-dimethylcyclohex-2-en-1-yl)-1-phenylethanone $2 \mathrm{~h}$ : $(2 E, 7 E)$ -5,5-Dimethyl-1,9-diphenylnona-2,7-diene-1,9-dione (33.2 mg, $0.1 \mathrm{mmol}$ ), 2mercaptophenol T2 (5.2 mg, $0.04 \mathrm{mmol})$, catalyst C5 (12.6 mg, $0.02 \mathrm{mmol})$ and $\mathrm{K}_{2} \mathrm{CO}_{3}(8.4 \mathrm{mg}, 0.06 \mathrm{mmol})$ were stirred in toluene $(1 \mathrm{~mL})$ at $\mathrm{rt}$ for $72 \mathrm{~h}$. After completion, purification by flash chromatography on silica gel $(\mathrm{EtOAc} / \mathrm{petroleum}$ ether $=1 / 40)$ gave 2h.<smiles>O=C(CC1COCC=C1C(=O)c1ccccc1)c1ccccc1</smiles>

(S)-2-(4-Benzoyl-3,6-dihydro-2H-pyran-3-yl)-1-phenylethanone $\quad \mathbf{2 i}: \quad\left(2 E, 2^{\prime} E\right)-$ 4,4'-Oxybis(1-phenylbut-2-en-1-one) (30.6 mg, $0.1 \mathrm{mmol}), 2$-mercaptophenol T2 (5.2 mg, $0.04 \mathrm{mmol})$, catalyst $\mathbf{C 4}(12.6 \mathrm{mg}, 0.02 \mathrm{mmol})$ and $\mathrm{K}_{2} \mathrm{CO}_{3}(8.4 \mathrm{mg}, 0.06$ mmol) were stirred in toluene $(1 \mathrm{~mL})$ at $\mathrm{rt}$ for $48 \mathrm{~h}$. After completion, purification by flash chromatography on silica gel $($ EtOAc/petroleum ether $=1 / 10)$ gave $\mathbf{2 i}$.<smiles>Cc1ccc(C(=O)C[C@H]2CCCC=C2C(=O)c2ccc([N+](=O)[O-])cc2)cc1</smiles>

(S)-2-(2-(4-Nitrobenzoyl)cyclohex-2-en-1-yl)-1-(p-tolyl)ethanone 2j: (2E,7E)-1-(4-Nitrophenyl)-9-(p-tolyl)nona-2,7-diene-1,9-dione (36.3 mg, $0.1 \mathrm{mmol})$, 2-mercaptophenol T2 (5.2 $\mathrm{mg}, 0.04 \mathrm{mmol})$, catalyst $\mathbf{C 5}$ (12.6 mg, $0.02 \mathrm{mmol})$ and $\mathrm{K}_{2} \mathrm{CO}_{3}(8.4 \mathrm{mg}, 0.06 \mathrm{mmol})$ were stirred in toluene $(1 \mathrm{~mL})$ at $\mathrm{rt}$ for $48 \mathrm{~h}$. After completion, purification by flash chromatography on silica gel $($ EtOAc/petroleum ether $=1 / 40)$ gave $\mathbf{2} \mathbf{j}$.

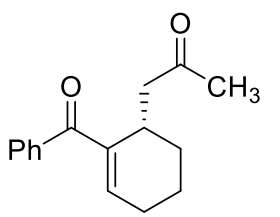

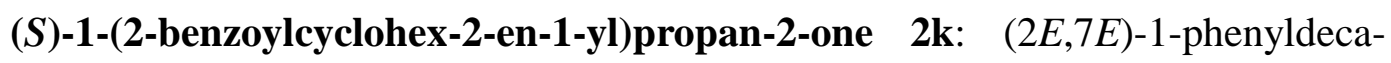
2,7-diene-1,9-dione (24.2 mg, $0.1 \mathrm{mmol}$ ), 2-mercaptophenol T2 (5.2 mg, 0.04 mmol), catalyst $\mathbf{C 5}$ (12.6 mg, $0.02 \mathrm{mmol})$ and $\mathrm{K}_{2} \mathrm{CO}_{3}(8.4 \mathrm{mg}, 0.06 \mathrm{mmol})$ were stirred in toluene $(1 \mathrm{~mL})$ at $\mathrm{rt}$ for $48 \mathrm{~h}$. After completion, purification by flash chromatography on 
silica gel $($ EtOAc/petroleum ether $=1 / 30)$ gave $\mathbf{2 k}$.

\section{General procedure for intramolecular $\mathrm{RC}$ reaction of bis(enones) 3}

General procedure: Bis(enones) $3(0.1 \mathrm{mmol})$, chiral thiol T4 $(0.01 \mathrm{mmol})$, catalyst $\mathbf{C 6}(0.01 \mathrm{mmol})$ and $\mathrm{K}_{2} \mathrm{CO}_{3}(0.06 \mathrm{mmol})$ were added into a vial equipped with a magnetic stir bar. Toluene $(1.0 \mathrm{~mL})$ was added. The mixture was stirred at room temperature for $24 \mathrm{~h}$. Purification by flash chromatography on silica gel (EtOAc/petroleum ether =1:40) gave the product 4 .

General procedure for racemic intramolecular $\mathbf{R C}$ reaction: Bis(enones) 3 (0.05 mmol), 2mercaptophenol T2 $(0.02 \mathrm{mmol}), \mathrm{TBAB}(0.01 \mathrm{mmol})$ and $\mathrm{K}_{2} \mathrm{CO}_{3}(0.03 \mathrm{mmol})$ were added into a vial equipped with a magnetic stir bar. Toluene $(1.0 \mathrm{~mL})$ was added. The mixture was stirred at $50{ }^{\circ} \mathrm{C}$ for $8 \mathrm{~h}$. Purification by flash chromatography on silica gel (EtOAc/petroleum ether $=1: 40$ ) gave the racemic product 4 .

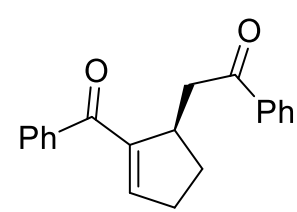

(R)-2-(2-Benzoylcyclopent-2-en-1-yl)-1-phenylethanone $\quad 4 a: \quad(2 E, 6 E)-1,8-$ Diphenylocta-2,6-diene-1,8-dione (29.0 mg, 0.1 mmol), $(R)$-bis-ortho-mercapto phenol T4 (3.5 mg, $0.01 \mathrm{mmol})$, catalyst $\mathbf{C 6}(7.8 \mathrm{mg}, 0.01 \mathrm{mmol})$ and $\mathrm{K}_{2} \mathrm{CO}_{3}(8.4$ $\mathrm{mg}, 0.06 \mathrm{mmol})$ were stirred in toluene $(1 \mathrm{~mL})$ at $\mathrm{rt}$ for $24 \mathrm{~h}$. After completion, purification by flash chromatography on silica gel $($ EtOAc/petroleum ether $=1 / 40)$ gave $\mathbf{4 a}$.

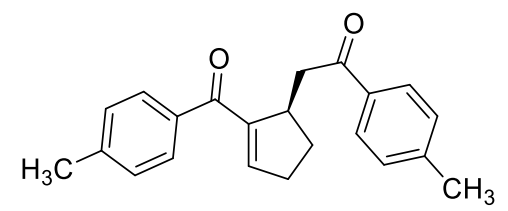

(R)-2-(2-(4-Methylbenzoyl)cyclopent-2-en-1-yl)-1-(p-tolyl)

ethanone 4b: $(2 E, 6 E)-1,8$-Di( $p$-tolyl)octa-2,6-diene-1,8-dione $(31.8$ mmol), catalyst $\mathbf{C 6}(7.8 \mathrm{mg}, 0.01 \mathrm{mmol})$ and $\mathrm{K}_{2} \mathrm{CO}_{3}(8.4 \mathrm{mg}, 0.06 \mathrm{mmol})$ were stirred in toluene (1 $\mathrm{mL}$ ) at $\mathrm{rt}$ for $24 \mathrm{~h}$. After completion, purification by flash chromatography on silica gel $($ EtOAc/petroleum ether $=1 / 40)$ gave $\mathbf{4 b}$.

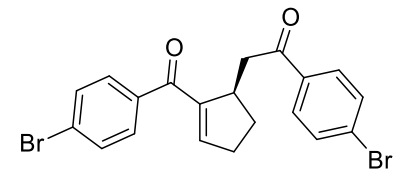

(R)-2-(2-(4-Bromobenzoyl)cyclopent-2-en-1-yl)-1-(4-bromophenyl) ethanone 4c: (2E,6E)-1,8-Di(4-bromophenyl)octa-2,6-diene-1,8-dione (44.6 mg, $0.1 \mathrm{mmol}),(R)$-bis-ortho-mercaptophenol T4 (3.5 mg, $0.01 \mathrm{~m} \mathrm{~mol})$, catalyst C6 (7.8 mg, 
$0.01 \mathrm{mmol})$ and $\mathrm{K}_{2} \mathrm{CO}_{3}(8.4 \mathrm{mg}, 0.06 \mathrm{mmol})$ were stirred in toluene $(1 \mathrm{~mL})$ at $\mathrm{rt}$ for $12 \mathrm{~h}$. After completion, purification by flash chromatography on silica gel $($ EtOAc/petroleum ether $=1 / 40)$ gave $4 c$.

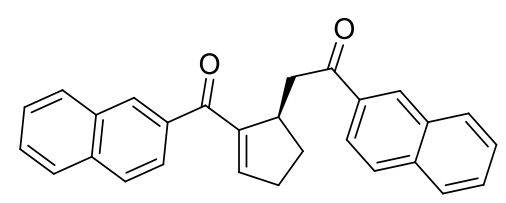

(R)-2-(2-(2-Naphthoyl)cyclopent-2-en-1-yl)-1-(naphthalen-2-yl)

ethanone 4d: (2E,6E)-1,8-Di(naphthalen-2-yl)octa-2,6-diene-1,8dione (39.0 mg, $0.1 \mathrm{mmol}),(R)$-bis-ortho-mercaptophenol T4 (2.6 mg, $0.01 \mathrm{mmol})$, catalyst $\mathbf{C 6}(7.8 \mathrm{mg}, 0.01 \mathrm{mmol})$ and $\mathrm{K}_{2} \mathrm{CO}_{3}(8.4 \mathrm{mg}, 0.06 \mathrm{mmol})$ were stirred in toluene $(1 \mathrm{~mL})$ at $\mathrm{rt}$ for $24 \mathrm{~h}$. After completion, purification by flash chromatography on silica gel $($ EtOAc/petroleum ether $=1 / 40)$ gave $\mathbf{4 d}$.

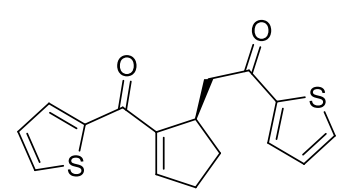

\section{(R)-1-(Thiophen-2-yl)-2-(2-(thiophene-2-carbonyl)cyclopent-2-en-1-yl)}

ethanone 4e: $(2 E, 6 E)-1,8$-Di(thiophen-2-yl)octa-2,6-diene-1,8-dione $(30.2 \mathrm{mg}$, $0.1 \mathrm{mmol}),(R)$-bis-ortho-mercaptophenol T4 (3.5 $\mathrm{mg}, 0.01 \mathrm{mmol})$, catalyst C6 (7.8 mg, $0.01 \mathrm{mmol})$ and $\mathrm{K}_{2} \mathrm{CO}_{3}(8.4 \mathrm{mg}, 0.06 \mathrm{mmol})$ were stirred in toluene $(1 \mathrm{~mL})$ at $\mathrm{rt}$ for $24 \mathrm{~h}$. After completion, purification by flash chromatography on silica gel $($ EtOAc/petroleum ether $=1 / 40)$ gave 4e. 
5. Screening conditions for desymmetric intramolecular RC reaction of bis(enones) 5 a

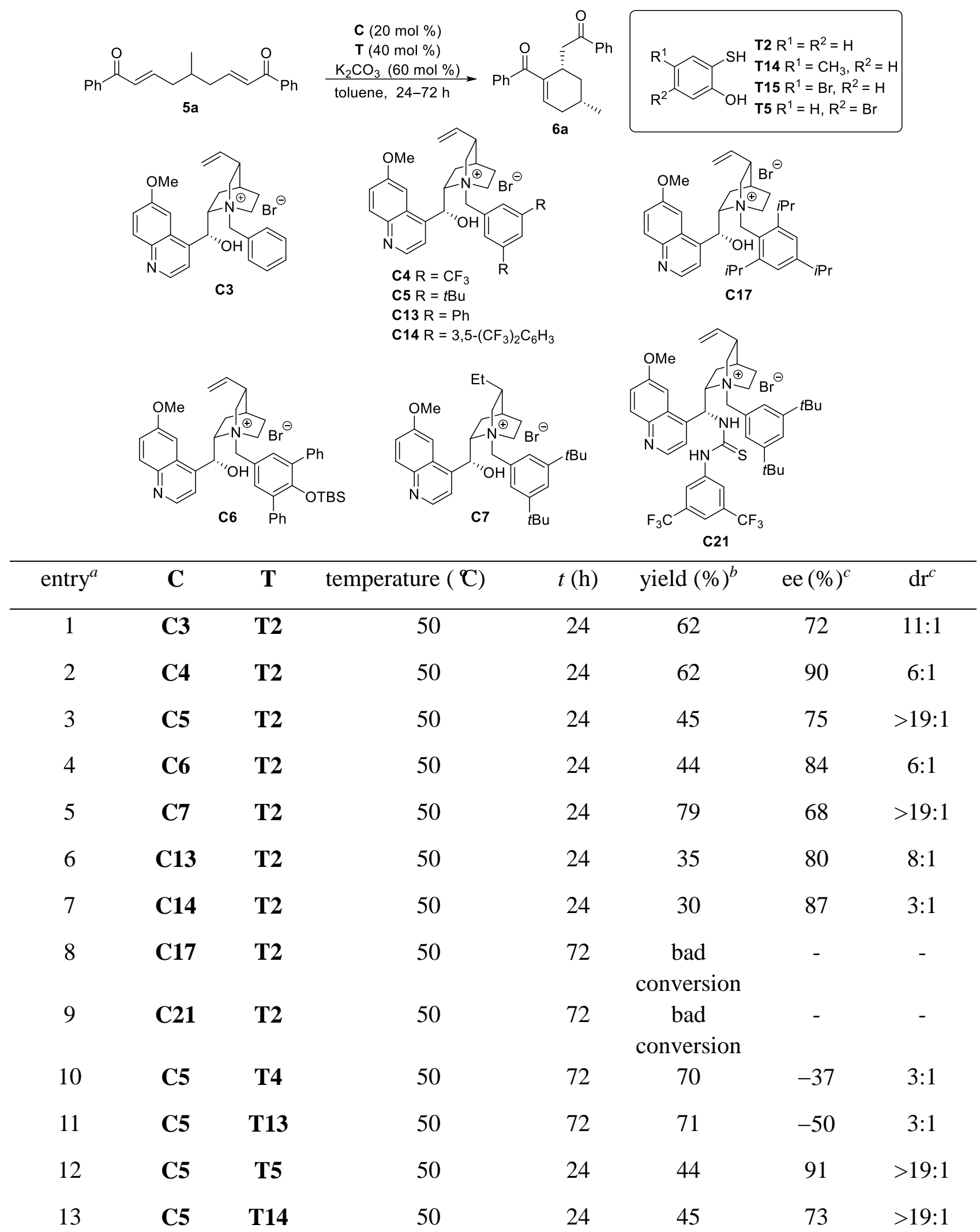


rt

72

76

$92 \quad>19: 1$

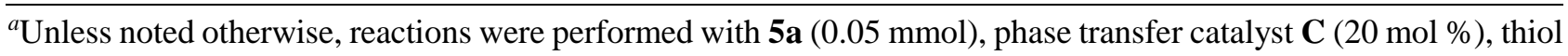
$\mathbf{T}(40 \mathrm{~mol} \%)$ and $\mathrm{K}_{2} \mathrm{CO}_{3}(60 \mathrm{~mol} \%)$ in toluene $(1 \mathrm{~mL}) .{ }^{b}$ Yield of the isolated product. ${ }^{c}$ Determined by HPLC analysis on a chiral stationary phase.

\section{General procedure for desymmetric intramolecular $\mathrm{RC}$ reaction of bis(enones)}

\section{5}
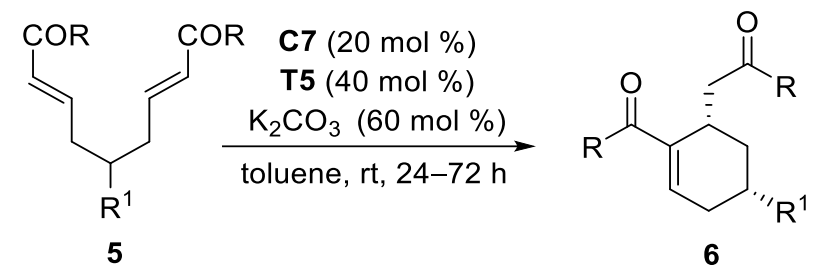

General procedure: Bis(enones) 5 (0.1 mmol), 2-mercaptophenol T5 (0.04 mmol), catalyst C7 (0.02 mmol) and $\mathrm{K}_{2} \mathrm{CO}_{3}(0.06 \mathrm{mmol})$ were added into a vial equipped with a magnetic stir bar. Toluene $(1.0 \mathrm{~mL})$ was added. The mixture was stirred at room temperature for 24 to $72 \mathrm{~h}$. Purification by flash chromatography on silica gel $($ EtOAc/petroleum ether $=1 / 40)$ gave the product 6.

General procedure for racemic desymmetric intramolecular $\mathbf{R C}$ reaction: Bis(enones) $\mathbf{5}(0.05$ mmol), 2-mercaptophenol T2 $(0.02 \mathrm{mmol})$, TBAB $(0.01 \mathrm{mmol})$ and $\mathrm{K}_{2} \mathrm{CO}_{3}(0.03 \mathrm{mmol})$ were added into a vial equipped with a magnetic stir bar. Toluene $(1.0 \mathrm{~mL})$ was added. The mixture was stirred at $50{ }^{\circ} \mathrm{C}$ for $8 \mathrm{~h}$. Purification by flash chromatography on silica gel $($ EtOAc/petroleum ether $=1 / 40)$ gave the racemic product $\mathbf{6}$.<smiles>C[C@@H]1CC=C(C(=O)c2ccccc2)C(CC(=O)c2ccccc2)C1</smiles>

(2E,7E)-5-Methyl-1,9-diphenylnona-2,7-diene-1,9-dione (31.8 mg, 0.1mmol), 5-

Br-2-mercptophenol T5 (8.1 mg, $0.04 \mathrm{mmol})$, catalyst C7 (12.5 mg, $0.02 \mathrm{mmol})$ and $\mathrm{K}_{2} \mathrm{CO}_{3}(8.4 \mathrm{mg}, 0.06 \mathrm{mmol})$ were stirred in toluene $(1 \mathrm{~mL})$ at $\mathrm{rt}$ for $72 \mathrm{~h}$. After completion, purification by flash chromatography on silica gel (EtOAc/petroleum ether $=1 / 40)$ gave $\mathbf{6 a}$. 
<smiles>Cc1ccc(C(=O)C[C@H]2C[C@H](C)CC=C2C(=O)c2ccc(C)cc2)cc1</smiles>

((1S,5R)-5-Methyl-2-(4-methylbenzoyl)cyclohex-2-en-1-yl)-1(p-tolyl)ethanone 6b: (2E,7E)-5-Methyl-1,9-di-p-tolylnona-2,7diene-1,9-dione (34.6 mg, $0.1 \mathrm{mmol}$ ), 5-Br-2-mercptophenol T5 (8.1 mg, $0.04 \mathrm{mmol})$, catalyst $\mathbf{C 7}(12.5 \mathrm{mg}, 0.02 \mathrm{mmol})$ and $\mathrm{K}_{2} \mathrm{CO}_{3}$ $(8.4 \mathrm{mg}, 0.06 \mathrm{mmol})$ were stirred in toluene $(1 \mathrm{~mL})$ at $\mathrm{rt}$ for $72 \mathrm{~h}$. After completion, purification by flash chromatography on silica gel (EtOAc/petroleum ether $=1 / 40)$ gave $\mathbf{6 b}$.<smiles>COc1cccc(C(=O)CC2CC(C)CC=C2C(=O)c2cccc(OC)c2)c1</smiles>

((1S,5R)-2-(3-Methoxybenzoyl)-5-methylcyclohex-2-en-1-yl)1-(3-methoxyphenyl)ethanone 6c: $(2 E, 7 E)-1,9-B i s$ (3-methoxy phenyl)-5-methylnona-2,7-diene-1,9-dione (37.8 mg, $0.1 \mathrm{mmol})$, 5-Br-2-mercptophenol T5 (8.1 mg, $0.04 \mathrm{mmol})$, catalyst C7 (12.5 $\mathrm{mg}, 0.02 \mathrm{mmol})$ and $\mathrm{K}_{2} \mathrm{CO}_{3}(8.4 \mathrm{mg}, 0.06 \mathrm{mmol})$ were stirred in toluene $(1 \mathrm{~mL})$ at $\mathrm{rt}$ for $72 \mathrm{~h}$. After completion, purification by flash chromatography on silica gel $($ EtOAc/petroleum ether $=1 / 40)$ gave 6c.<smiles>C[C@H]1CC=C(C(=O)c2ccc(Br)cc2)C(CC(=O)c2ccc(Br)cc2)C1</smiles>

((1S,5R)-2-(4-Bromobenzoyl)-5-methylcyclohex-2-en-1-yl)-1-(4bromophenyl)ethanone $\quad \mathbf{6 d}: \quad(2 E, 7 E)-1,9-\operatorname{Bis}(4-$ bromophenyl)-5methyl nona-2,7-diene-1,9-dione (47.4 mg, $0.1 \mathrm{mmol}), 5-\mathrm{Br}-2-$ mercptophenol T5 (8.1 mg, $0.04 \mathrm{mmol})$, catalyst C7 (12.5 mg, $0.02 \mathrm{mmol})$ and $\mathrm{K}_{2} \mathrm{CO}_{3}(8.4 \mathrm{mg}, 0.06$ mmol) were stirred in toluene $(1 \mathrm{~mL})$ at $\mathrm{rt}$ for $48 \mathrm{~h}$. After completion, purification by flash chromatography on silica gel $($ EtOAc/petroleum ether $=1 / 60)$ gave $\mathbf{6 d}$.<smiles>C[C@H]1CC=C(C(=O)c2cccc(Cl)c2)C(CC(=O)c2cccc(Cl)c2)C1</smiles>

((1S,5R)-2-(3-Chlorobenzoyl)-5-methylcyclohex-2-en-1-yl)-1-(3chlorophenyl)ethanone $\quad \mathbf{6 e} \quad(2 E, 7 E)-1,9-\operatorname{Bis}(3-c h l o r o p h e n y l)-5-$ methyl nona-2,7-diene-1,9-dione (38.6 mg, $0.1 \mathrm{mmol}$ ), 5-Br-2mercptophenol T5 $(8.1 \mathrm{mg}, 0.04 \mathrm{mmol})$, catalyst $\mathbf{C 7}(12.5 \mathrm{mg}, 0.02 \mathrm{mmol})$ and $\mathrm{K}_{2} \mathrm{CO}_{3}(8.4 \mathrm{mg}, 0.06$ mmol) were stirred in toluene $(1 \mathrm{~mL})$ at $\mathrm{rt}$ for $24 \mathrm{~h}$. After completion, purification by flash chromatography on silica gel (EtOAc/petroleum ether $=1 / 40)$ gave $6 \mathbf{e}$. 
<smiles>C[C@H]1CC=C(C(=O)c2ccc3ccccc3c2)[C@H](CC(=O)c2ccc3ccccc3c2)C1</smiles>

((1S,5R)-2-(2-Naphthoyl)-5-methylcyclohex-2-en-1-yl)-1(naphthalen-2-yl)ethanone 6f: $(2 E, 7 E)-5-M e t h y l-1,9-d i(n a p h t h a l e n$ -2-yl) nona-2,7-diene-1,9-dione (20.9mg, $0.1 \mathrm{mmol})$, 5-Br-2-mercpto phenol T5 (8.1 mg, $0.04 \mathrm{mmol})$, catalyst C7 (12.5 mg, $0.02 \mathrm{mmol})$ and $\mathrm{K}_{2} \mathrm{CO}_{3}(8.4 \mathrm{mg}, 0.06 \mathrm{mmol})$ were stirred in toluene $(1 \mathrm{~mL})$ at $\mathrm{rt}$ for $72 \mathrm{~h}$. After completion, purification by flash chromatography on silica gel $($ EtOAc/petroleum ether $=1 / 40)$ gave $6 \mathbf{f}$.<smiles>C[C@H]1CC=C(C(=O)c2cccs2)C(CC(=O)c2cccs2)C1</smiles>

((1S,5R)-5-Methyl-2-(thiophene-2-carbonyl)cyclohex-2-en-1-yl)-1(thiophen-2-yl)ethan-1-one 6g: (2E,7E)-5-Methyl-1,9-di(thiophen-2-yl)nona2,7- diene-1,9-dione (33.1 mg, $0.1 \mathrm{mmol})$, 5-Br-2-mercptophenol T5 (8.1 mg, $0.04 \mathrm{mmol})$, catalyst $\mathbf{C 7}(12.5 \mathrm{mg}, 0.02 \mathrm{mmol})$ and $\mathrm{K}_{2} \mathrm{CO}_{3}(8.4 \mathrm{mg}, 0.06 \mathrm{mmol})$ were stirred in toluene $(1 \mathrm{~mL})$ at $\mathrm{rt}$ for $72 \mathrm{~h}$. After completion, purification by flash chromatography on silica gel $($ EtOAc/petroleum ether $=1 / 40)$ gave $\mathbf{6 g}$.<smiles>O=C(CC1CC(c2ccccc2)CC=C1C(=O)c1ccccc1)c1ccccc1</smiles>

((1R,3S)-4-Benzoyl-1,2,3,6-tetrahydro-[1,1'-biphenyl]-3-yl)-1-phenylethanone 6h: (2E,7E)-1,5,9-Triphenylnona-2,7-diene-1,9-dione (38.0 mg, $0.1 \mathrm{mmol}), 5$-Br-2mercptophenol T5 $(8.1 \mathrm{mg}, 0.04 \mathrm{mmol})$, catalyst C7 (12.5 mg, $0.02 \mathrm{mmol})$ and $\mathrm{K}_{2} \mathrm{CO}_{3}(8.4 \mathrm{mg}, 0.06 \mathrm{mmol})$ were stirred in toluene $(1 \mathrm{~mL})$ at $\mathrm{rt}$ for $48 \mathrm{~h}$. After completion, purification by flash chromatography on silica gel $($ EtOAc/petroleum ether $=1 / 40)$ gave 6h.<smiles>O=C(CC1CC(c2ccc(Cl)cc2)CC=C1C(=O)c1ccccc1)c1ccccc1</smiles>
((1R,3S)-4-Benzoyl-4'-chloro-1,2,3,6-tetrahydro-[1,1'-biphenyl]-3-yl)-1phenylethanone $\quad \mathbf{6 i}: \quad(2 E, 7 E)-5-(4-C h l o r o p h e n y l)-1,9-d i p h e n y l n o n a-2,7-$ diene-1,9-dione (20.7 mg, $0.1 \mathrm{mmol}$ ), 5-Br-2-mercptophenol T5 (8.1 mg, 0.04 mmol), catalyst $\mathbf{C} 7$ (12.5 mg, $0.02 \mathrm{mmol})$ and $\mathrm{K}_{2} \mathrm{CO}_{3}(8.4 \mathrm{mg}, 0.06 \mathrm{mmol})$ were stirred in toluene $(1 \mathrm{~mL})$ at $\mathrm{rt}$ for $48 \mathrm{~h}$. After completion, purification by flash chromatography on silica gel $($ EtOAc/petroleum ether $=1 / 20)$ gave $\mathbf{6 i}$. 
<smiles>CCOC(=O)c1ccccc1</smiles>

Ethyl-(1R,5S)-4-benzoyl-5-(2-oxo-2-phenylethyl)cyclohex-3-ene-1carboxylate 6j: (E)-Ethyl 6-oxo-2-((E)-4-oxo-4-phenylbut-2-en-1-yl)-6phenylhex-4-enoate (37.6 mg, $0.1 \mathrm{mmol}), 5-\mathrm{Br}-2-$ mercptophenol T5 $(8.1 \mathrm{mg}$, $0.04 \mathrm{mmol})$, catalyst $\mathbf{C 7}(12.5 \mathrm{mg}, 0.02 \mathrm{mmol})$ and $\mathrm{K}_{2} \mathrm{CO}_{3}(8.4 \mathrm{mg}, 0.06 \mathrm{mmol})$ were stirred in toluene $(1 \mathrm{~mL})$ at $\mathrm{rt}$ for $48 \mathrm{~h}$. After completion, purification by flash chromatography on silica gel $($ EtOAc/petroleum ether $=1 / 20)$ gave product $\mathbf{6 j}$.<smiles>O=C(C[C@H]1C[C@H](O[GaH2])CC=C1C(=O)c1ccccc1)c1ccccc1</smiles>

((1S,5R)-2-Benzoyl-5-((tert-butyldimethylsilyl)oxy)cyclohex-2-en-1-yl)-1phenylethanone 6k: (2E,7E)-5-((tert-Butyldimethylsilyl)oxy)-1,9-diphenylnona -2,7-diene-1,9-dione (43.4 mg, $0.1 \mathrm{mmol}), 5$-Br-2-mercptophenol T5 $(8.1 \mathrm{mg}$, $0.04 \mathrm{mmol})$, catalyst $\mathbf{C} 7(12.5 \mathrm{mg}, 0.02 \mathrm{mmol})$ and $\mathrm{K}_{2} \mathrm{CO}_{3}(8.4 \mathrm{mg}, 0.06 \mathrm{mmol})$ were stirred in toluene $(1 \mathrm{~mL})$ at $\mathrm{rt}$ for $36 \mathrm{~h}$. After completion, purification by flash chromatography on silica gel $($ EtOAc/petroleum ether $=1 / 20)$ gave product $\mathbf{6 k}$.<smiles>CC(C)C[C@H]1CC=C(C(=O)c2ccccc2)C(CC(=O)c2ccccc2)C1</smiles>

((1S,5R)-2-Benzoyl-5-isobutylcyclohex-2-en-1-yl)-1-phenylethanone 6l: (2E,7E)-5-Isobutyl-1,9-diphenylnona-2,7-diene-1,9-dione (17.3 mg, $0.1 \mathrm{mmol}), 5$ Br-2-mercptophenol T5 (8.1 mg, $0.04 \mathrm{mmol})$, catalyst C7 (12.5 mg, $0.02 \mathrm{mmol})$ and $\mathrm{K}_{2} \mathrm{CO}_{3}(8.4 \mathrm{mg}, 0.06 \mathrm{mmol})$ were stirred in toluene $(1 \mathrm{~mL})$ at $\mathrm{rt}$ for $36 \mathrm{~h}$. After completion, purification by flash chromatography on silica gel $($ EtOAc/petroleum ether $=1 / 40)$ gave product $6 \mathbf{l}$.<smiles>O=C(CC1CC(c2ccc(Cl)cc2)CC=C1C(=O)c1ccc2ccccc2c1)c1ccc2ccccc2c1</smiles>

$((1 R, 3 S)-4-(2-N a p h t h o y l)-4 '$-chloro-1,2,3,6-tetrahydro-[1,1'biphenyl]-3-yl)-1-(naphthalen-2-yl)ethan-1-one 6m: (2E,7E)-5-(4Chlorophenyl)-1,9-di(naphthalen-2-yl)nona-2,7-diene-1,9-dione (51.4 mg, $0.1 \mathrm{mmol})$, 5-Br-2-mercptophenol T5 (8.1 mg, $0.04 \mathrm{mmol})$, catalyst $\mathbf{C 7}(12.5 \mathrm{mg}, 0.02 \mathrm{mmol})$ and $\mathrm{K}_{2} \mathrm{CO}_{3}(8.4 \mathrm{mg}, 0.06 \mathrm{mmol})$ were stirred in $\mathrm{CHCl}_{3}(1 \mathrm{~mL})$ at rt for $48 \mathrm{~h}$. After completion, purification by flash chromatography on silica gel (EtOAc/petroleum ether $=1 / 30$ ) gave $6 \mathbf{m}$. 


\section{More exploration on intramolecular $\mathrm{RC}$ reaction of diversely structured}

\section{substrates}

5,5-Disubstituted bis(enones) 7 could construct chiral the product with a quaternary stereogenic carbon. The desired RC product $\mathbf{8}$ was obtained with inferior ee and dr values when 2-mercaptophenol T2 and C5 were applied (entry 1), whereas better results were delivered under the catalysis of chiral PTC C6 and chiral mercaptophenol T4 (entry 2).

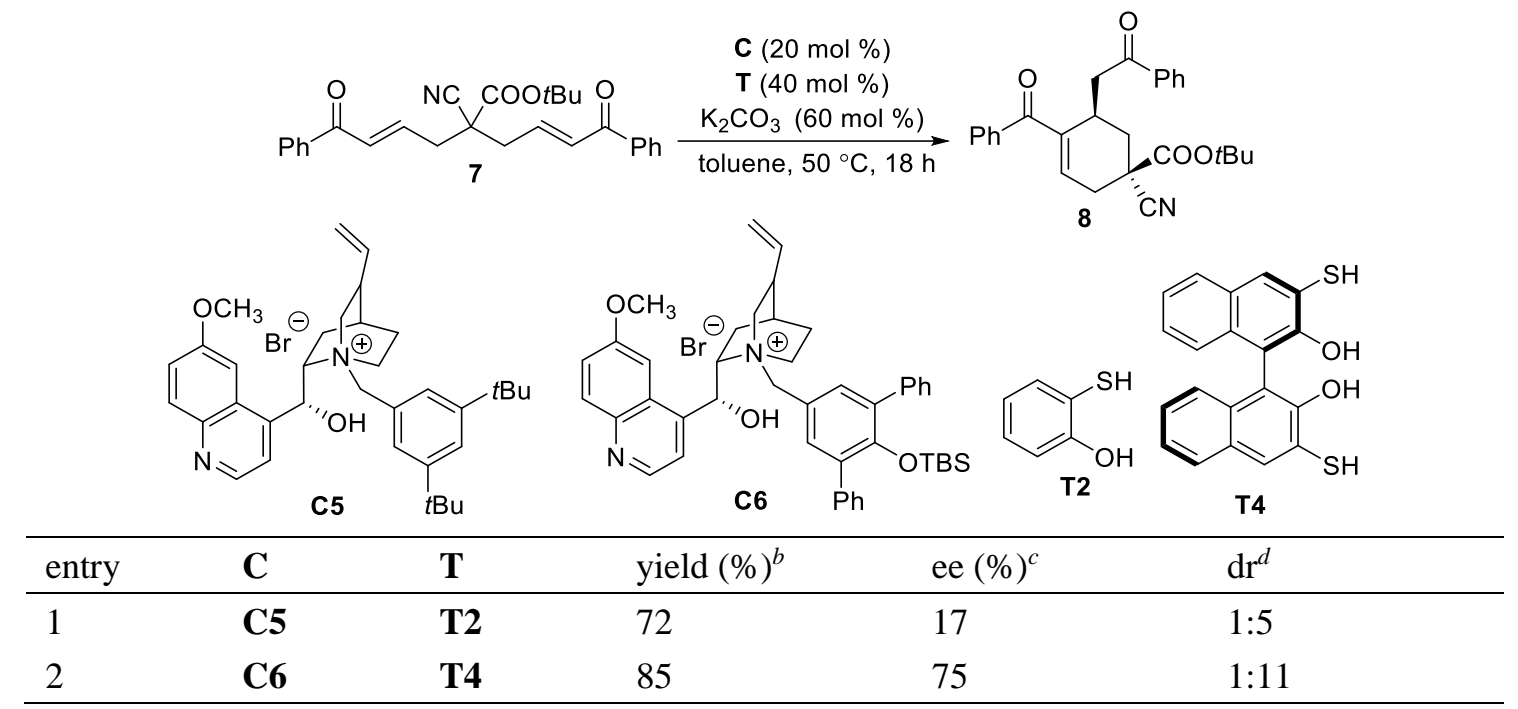

${ }^{a}$ Unless noted otherwise, reactions were performed with $7(0.1 \mathrm{mmol})$, phase transfer catalysts $\mathbf{C}(20 \mathrm{~mol} \%)$, Thiol (40 mol \%) and $\mathrm{K}_{2} \mathrm{CO}_{3}(60 \mathrm{~mol} \%)$ in toluene $(1 \mathrm{~mL}) .{ }^{b}$ Yield of the isolated product. ${ }^{c}$ Determined by HPLC analysis on a chiral stationary phase. ${ }^{d}$ Determined by ${ }^{1} \mathrm{H}$ NMR analysis.<smiles>CCOC(=O)C1(C#N)CC=C(C(=O)c2ccccc2)C(CC(=O)c2ccccc2)C1</smiles>

tert-Butyl (1S,5R)-4-benzoyl-1-cyano-5-(2-oxo-2-phenylethyl)cyclohex- 3ene-1-carboxylate 8: tert-Butyl (E)-2-cyano-6-oxo-2-((E)-4-oxo-4-phenyl but-2-en-1-yl)-6-phenylhex-4-enoate $5(42.9 \mathrm{mg}, 0.1 \mathrm{mmol}),(R)$-bis-orthomercaptophenol T4 (14.1 mg, $0.04 \mathrm{mmol})$, catalyst C6 (15.6 mg, $0.02 \mathrm{mmol})$

and $\mathrm{K}_{2} \mathrm{CO}_{3}(8.4 \mathrm{mg}, 0.06 \mathrm{mmol})$ were stirred in toluene $(1 \mathrm{~mL})$ at $50{ }^{\circ} \mathrm{C}$ for $18 \mathrm{~h}$. After completion, purification by flash chromatography on silica gel (EtOAc/petroleum ether $=1 / 20)$ gave $\mathbf{8}$.<smiles>O=C(C[C@H]1C[C@H]2CC[C@@H]1CC2)c1ccccc1</smiles>

2-((1S,2R,5R)-3-Benzoylbicyclo[3.2.1] oct-3-en-2-yl)-1-phenylethan-1-one 10: 3,3'- (Cyclopentane-1,3-diyl)bis(1-phenylprop-2-en-1-one) 9 (33.0 mg, $0.1 \mathrm{mmol}$, as a cis/trans mixture, but the ratio might be various from different preparation reactions), 2-mercaptophenol T2 (5.2 $\mathrm{mg}, 0.04 \mathrm{mmol})$, catalyst $\mathbf{C 5}$ (12.6 mg, 0.02 
$\mathrm{mmol})$ and $\mathrm{K}_{2} \mathrm{CO}_{3}(13.8 \mathrm{mg}, 0.1 \mathrm{mmol})$ were stirred in toluene $(1 \mathrm{~mL})$ at $50{ }^{\circ} \mathrm{C}$ for $48 \mathrm{~h}$. After completion, purification by flash chromatography on silica gel $(\mathrm{EtOAc} /$ petroleum ether $=1 / 40)$ gave 10.<smiles>CC1=CC(=O)C2=CCCC[C@H]2C1</smiles>

(S)-3-Methyl-4a,5,6,7-tetrahydronaphthalen-1(4H)-one 11: $(3 E, 8 E)$-Undeca3,8-diene-2,10-dione 11 (18.0 mg, $0.1 \mathrm{mmol}$ ), 2-mercaptophenol T2 (5.2 mg, 0.04 mmol), catalyst $\mathbf{C 5}$ (12.6 mg, $0.02 \mathrm{mmol})$ and $\mathrm{K}_{2} \mathrm{CO}_{3}(27.6 \mathrm{mg}, 0.2 \mathrm{mmol})$ were stirred in toluene $(1 \mathrm{~mL})$ at $60{ }^{\circ} \mathrm{C}$ for $48 \mathrm{~h}$. After completion, purification by flash chromatography on silica gel $($ EtOAc/petroleum ether $=1 / 20)$ to give 11 .

A chalcone substrate possessing an enolate moiety was unsuccessful in the intramolecular RC reaction. $o$-Nitrovinyl phenoxyacrylates $12 \mathbf{a}$ was successful to give the RC product in $85 \%$ yield but in a racemic form under the catalysis of PTC C5 and T2.<smiles>CCOC(=O)/C=C/Oc1ccccc1/C=C/C(=O)Oc1ccccc1/C=C/Oc1ccccc1</smiles>

$12 \mathrm{a}$

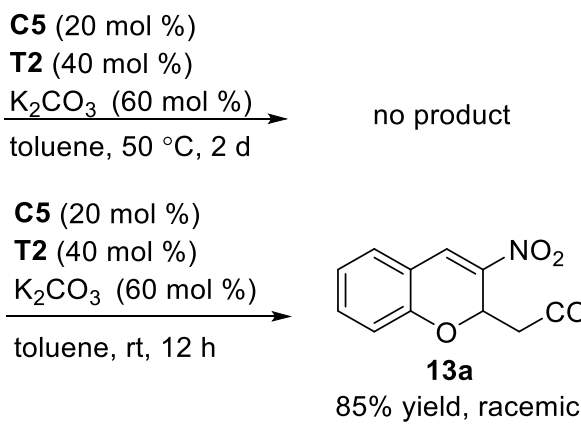

$13 a$

$85 \%$ yield, racemic

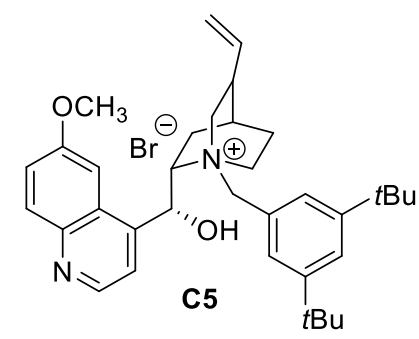

To our delight, the combination of thiol and chiral tertiary amine could give good enantiocontrol in the corresponding $\mathrm{RC}$ reaction, as summarized in the following table.

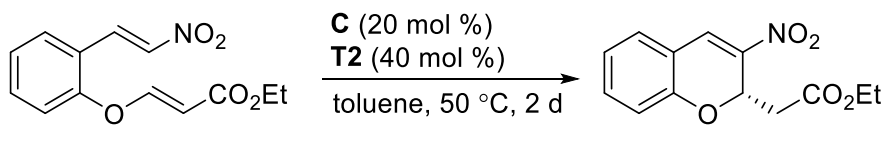

$12 a$

$13 a$<smiles>C=CC1CC2CCN1CC2[C@H](Nc1c(Nc2cc(C(F)(F)F)cc(C(F)(F)F)c2)c(=O)c1=O)c1ccnc2ccc(OC)cc12</smiles><smiles>C=CC1CC2CCN1CC2[C@H](NC(=S)Nc1cc(C(F)(F)F)cc(C(F)(F)F)c1)c1ccnc2ccccc12</smiles>

cinchonine C22 cinchonidine C23 quinine C24 quinidine $\quad$ C25<smiles>Cc1ccc(NC(=S)N[C@H](c2ccccc2)[C@@H](c2ccccc2)N(C)C)cc1</smiles> 


\begin{tabular}{llll}
\hline entry $^{a}$ & C & yield $(\%)^{b}$ & ee $(\%)^{c}$ \\
\hline 1 & $\mathbf{C 2 2}$ & 87 & 49 \\
2 & $\mathbf{C 2 3}$ & 86 & -40 \\
3 & $\mathbf{C 2 4}$ & 79 & -44 \\
4 & $\mathbf{C 2 5}$ & 80 & 45 \\
5 & $\mathbf{C 2 6}$ & 88 & 76 \\
6 & $\mathbf{C 2 7}$ & 90 & 85 \\
$\mathbf{7}$ & $\mathbf{C 9}$ & $\mathbf{8 1}$ & $\mathbf{- 9 1}$ \\
8 & $\mathbf{C 2 8}$ & 89 & 86 \\
\hline
\end{tabular}

${ }^{a}$ Unless noted otherwise, reactions were performed with 12a $(0.1 \mathrm{mmol})$, tertiary amine catalyst $\mathbf{C}(20 \mathrm{~mol} \%)$, thiol $\mathbf{T}(40 \mathrm{~mol} \%)$ and $\mathrm{K}_{2} \mathrm{CO}_{3}(60 \mathrm{~mol} \%)$ in toluene $(1 \mathrm{~mL})$ at $50{ }^{\circ} \mathrm{C} .{ }^{b}$ Yield of the isolated product. ${ }^{c}$ Determined by HPLC analysis on a chiral stationary phase.<smiles>CCOC(=O)C1=Cc2ccccc2OC1CCOC</smiles>

Ethyl (R)-2-(3-nitro-2H-chromen-2-yl)acetate 13a: Ethyl $(E)-3-(2-((E)-2-$ nitrovinyl)phenoxy)acrylate 12a (26.3 mg, $0.1 \mathrm{mmol}), 2$-mercaptophenol T2 (5.2 $\mathrm{mg}, 0.04 \mathrm{mmol})$, catalyst $\mathbf{C 9}(7.8 \mathrm{mg}, 0.02 \mathrm{mmol})$ were dissolved in toluene $(1.0 \mathrm{~mL})$. Then the mixture was stirred at $50{ }^{\circ} \mathrm{C}$ for $48 \mathrm{~h}$. After completion, purification by flash chromatography on silica gel $($ EtOAc/petroleum ether $=1 / 40)$ gave 13a.<smiles>CC(=O)CC1Oc2ccccc2C=C1[N+](=O)[O-]</smiles>

Methyl (R)-2-(3-nitro-2H-chromen-2-yl)acetate 13b: Methyl (E)-3-(2-((E)2-nitrovinyl)phenoxy)acrylate $\mathbf{1 2 b}(24.9 \mathrm{mg}, 0.1 \mathrm{mmol}), 2$-mercaptophenol T2 (5.2 $\mathrm{mg}, 0.04 \mathrm{mmol})$, catalyst $\mathbf{C 9}(7.8 \mathrm{mg}, 0.02 \mathrm{mmol})$ were dissolved in toluene $(1.0 \mathrm{~mL})$. Then the mixture was stirred at $50{ }^{\circ} \mathrm{C}$ for $48 \mathrm{~h}$. After completion, purification by flash chromatography on silica gel $($ EtOAc/petroleum ether $=1 / 40)$ gave $\mathbf{1 3 b}$.

A bis(enones) substrate containing thioenoates was tried under the standard conditions. However, a bad conversion was observed even at $60{ }^{\circ} \mathrm{C}$, probably due to the low electrophilicity of the thioenoates.<smiles>[R11]Nc1ccccc1C(=O)C=CCCCC=CC(=O)CC</smiles>

C5 $(20 \mathrm{~mol} \%)$

T2 (40 $\mathrm{mol} \%)$

$\underset{\text { toluene, } 60{ }^{\circ} \mathrm{C}}{\stackrel{\mathrm{K}_{2} \mathrm{CO}_{3}(60 \mathrm{~mol} \%)}{\longrightarrow}}$

bad conversion<smiles>C=CC1CC2CCC1N(Cc1cc(C(C)(C)C)cc(C(C)(C)C)c1)C2[C@H](O)c1ccnc2ccc(OC)cc12</smiles>

C5 


\section{Transformations of products $2 \mathrm{c}$ and $6 \mathrm{~m}$}

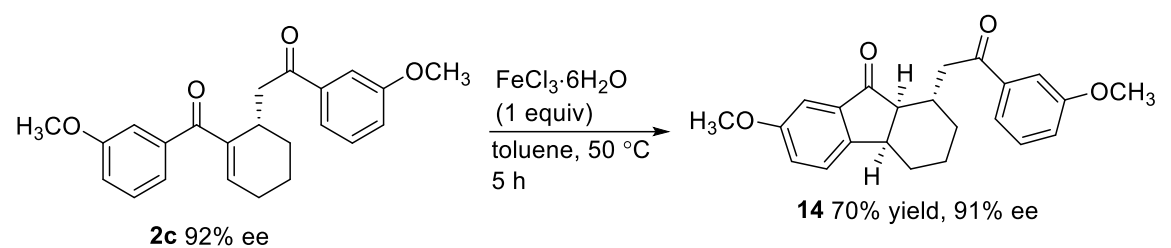

(1S,4aR,9aS)-7-Methoxy-1-(2-(3-methoxyphenyl)-2-oxoethyl)-1,2,3,4,4a,9a-hexahydro-9H-

fluoren-9-one 14: (S)-2-(2-(3-Methoxybenzoyl)cyclohex-2-en-1-yl)-1-(3-methoxyphenyl)ethan-1one $2 c(36.4 \mathrm{mg}, 0.1 \mathrm{mmol}), \mathrm{FeCl}_{3} \cdot 6 \mathrm{H}_{2} \mathrm{O}(27.0 \mathrm{mg}, 0.1 \mathrm{mmol})$ were stirred in toluene $(1 \mathrm{~mL})$ at 50 ${ }^{\circ} \mathrm{C}$ for $5 \mathrm{~h}$. After completion, purification by flash chromatography on silica gel (EtOAc/petroleum ether $=1 / 40)$ gave product 14 .

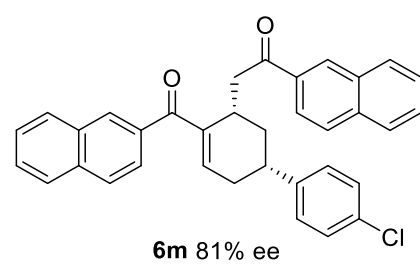

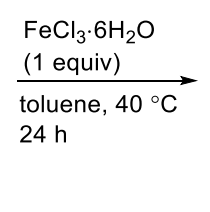

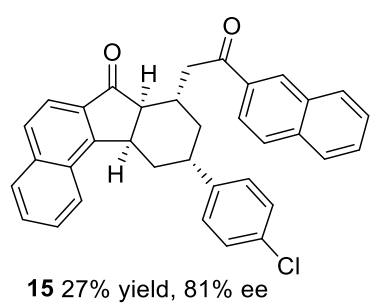

$1527 \%$ yield, $81 \%$ ee

(7aS,8S,10S,11aR)-10-(4-Chlorophenyl)-8-(2-(naphthalen-2-yl)-2-oxoethyl)-7a,8,9,10,11,11ahexahydro-7H-benzo[c]fluoren-7-one $\quad 15$ : $\quad 2-((1 R, 3 S)-4-(2-n N a p h t h o y l)-4$ '-chloro-1,2,3,6tetrahydro-[1,1'-biphenyl]-3-yl)-1-(naphthalen-2-yl)ethan-1-one $\quad \mathbf{6 m} \quad(51.4 \quad \mathrm{mg}, \quad 0.1 \mathrm{mmol})$, $\mathrm{FeCl}_{3} \cdot 6 \mathrm{H}_{2} \mathrm{O}(27.0 \mathrm{mg}, 0.1 \mathrm{mmol})$ were stirred in toluene $(1.0 \mathrm{~mL})$ at $40{ }^{\circ} \mathrm{C}$ for $24 \mathrm{~h}$. After completion, purification by flash chromatography on silica gel (EtOAc/petroleum ether $=1 / 20)$ gave product 15 .

\section{Crystal data and structural refinement for enantiopure 8 and 15 (derivative of}

6m)

Procedure for the recrystallization of $\mathbf{8}$ : To a $10 \mathrm{~mL}$ tube containing $\mathbf{8}(10 \mathrm{mg})$ was added a 4:1 mixture of $n$-hexane and EtOAc (about $2 \mathrm{~mL}$ ). The mixture was heated until a clear solution was formed, which was kept aside for $24 \mathrm{~h}$ at room temperature to produce crystals. These crystals were subjected for single crystal XRD to determine the absolute configuration of $\mathbf{8}$. The data were collected by an Agilent Gemini equipped with a $\mathrm{Cu}$ radiation source $(\mathrm{K} \alpha=1.54184 \AA$ ) at 292(2) K. CCDC 2002926 (8) contains the supplementary crystallographic data for this paper. These data can be 
obtained free of charge via www.ccdc.cam.ac.uk/data_request/cif.

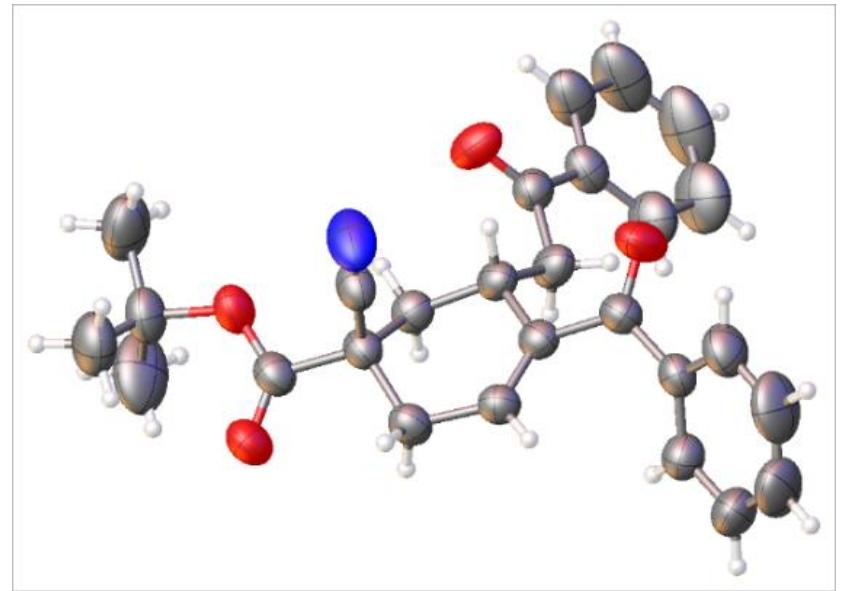<smiles>CCOC(=O)[C@]1(C#N)CC=C(C(=O)c2ccccc2)[C@H](CC(=O)c2ccccc2)C1</smiles>

8 (CCDC 2002926)

(ellipsoid contour probability 50\%)

Identification code

Empirical formula

Formula weight

Temperature/K

Crystal system

Space group

$\mathrm{a} / \AA$

$\mathrm{b} / \AA$

$\mathrm{c} / \AA$

$\alpha /^{\circ}$

$\beta /^{\circ}$

$\gamma /{ }^{\circ}$

Volume $/ \AA^{3}$

Z

$\rho_{\text {calc }} \mathrm{g} / \mathrm{cm}^{3}$

$\mu / \mathrm{mm}^{-1}$

$\mathrm{F}(000)$

Crystal size $/ \mathrm{mm}^{3}$

Radiation
8

$\mathrm{C}_{27} \mathrm{H}_{27} \mathrm{NO}_{4}$

429.49

$295.8(5)$

orthorhombic

$\mathrm{P} 2{ }_{1} 2{ }_{1}{ }_{1}$

$10.3861(2)$

11.8749(3)

19.6542(5)

90

90

90

2424.03(11)

4

1.177

0.633

912.0

$0.7 \times 0.35 \times 0.3$

$\operatorname{CuK} \alpha(\lambda=1.54184)$ 
$2 \Theta$ range for data collection/ ${ }^{\circ}$

Index ranges

Reflections collected

Independent reflections

Data/restraints/parameters

Goodness-of-fit on $\mathrm{F}^{2}$

Final $\mathrm{R}$ indexes $[\mathrm{I}>=2 \sigma(\mathrm{I})]$

Final $\mathrm{R}$ indexes [all data]

Largest diff. peak/hole / e $\AA^{-3}$

Flack parameter
9.632 to 145.208

$-12 \leq \mathrm{h} \leq 9,-14 \leq \mathrm{k} \leq 11,-19 \leq 1 \leq 24$

11446

$4704\left[R_{\text {int }}=0.0286, R_{\text {sigma }}=0.0268\right]$

$4704 / 0 / 292$

1.052

$\mathrm{R}_{1}=0.0646, \mathrm{w}_{2}=0.1640$

$\mathrm{R}_{1}=0.0677, \mathrm{wR}_{2}=0.1702$

$0.22 /-0.36$

$-0.06(14)$

Procedure for the recrystallization of 15: To a $10 \mathrm{~mL}$ tube containing 15 (10 $\mathrm{mg})$ was added a 3:1 mixture of $n$-hexane and 1,2-dimethoxyethane (about $2 \mathrm{~mL}$ ). The mixture was heated until a clear solution was formed, which was kept aside for $24 \mathrm{~h}$ at room temperature to produce crystals. These crystals were subjected for single crystal XRD to determine the absolute configuration of $\mathbf{1 5}$. The data were collected by an Agilent Gemini equipped with a $\mathrm{Cu}$ radiation source $(\mathrm{K} \alpha=1.54184 \AA)$ at 292(2) K. CCDC 2002927 (15) contains the supplementary crystallographic data for this paper. These data can be obtained free of charge via www.ccdc.cam.ac.uk/data_request/cif.

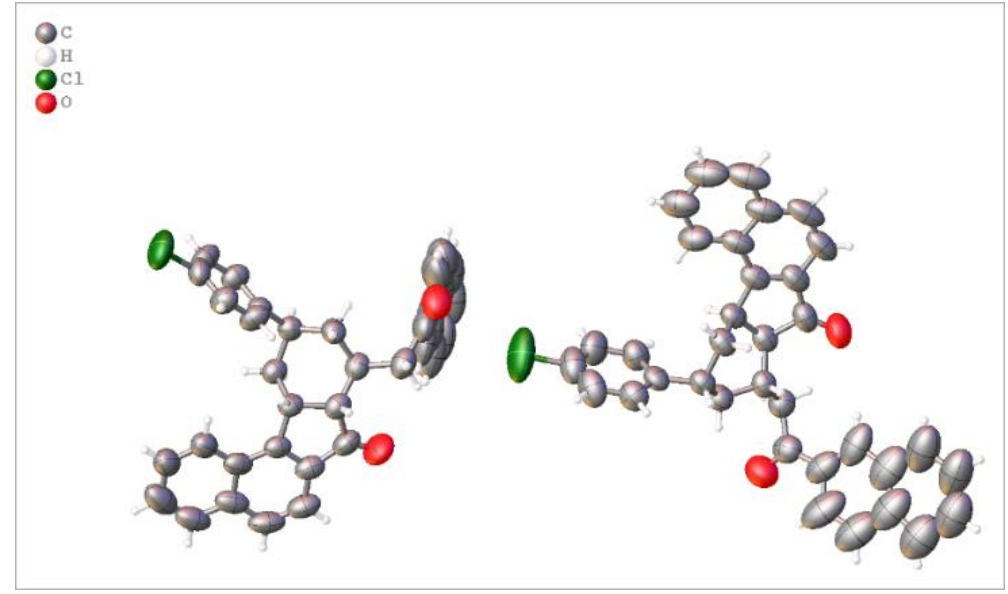

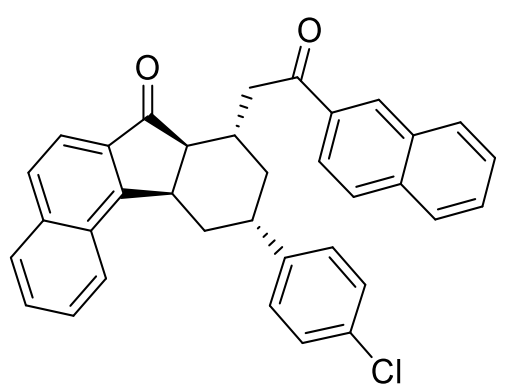

$15(\operatorname{CCDC} 2002927)$

(ellipsoid contour probability 50\%)

Identification code

Empirical formula

Formula weight
15

$\mathrm{C}_{35} \mathrm{H}_{27} \mathrm{ClO}_{2}$

515.01 
Temperature/K

Crystal system

Space group

$\mathrm{a} / \AA$

b/A

c/A

$\alpha /^{\circ}$

$\beta /^{\circ}$

$\gamma /{ }^{\circ}$

Volume $/ \AA^{3}$

Z

$\rho_{\text {calc }} \mathrm{g} / \mathrm{cm}^{3}$

$\mu / \mathrm{mm}^{-1}$

$\mathrm{F}(000)$

Crystal size/ $/ \mathrm{mm}^{3}$

Radiation

$2 \Theta$ range for data collection ${ }^{\circ}$

Index ranges

Reflections collected

Independent reflections

Data/restraints/parameters

Goodness-of-fit on $\mathrm{F}^{2}$

Final $R$ indexes $[\mathrm{I}>=2 \sigma(\mathrm{I})]$

Final $\mathrm{R}$ indexes [all data]

Largest diff. peak/hole / e $\AA^{-3}$

Flack parameter
292.8(9)

monoclinic

$\mathrm{P} 21$

13.6569(6)

$11.1976(7)$

17.9855(9)

90

$97.290(5)$

90

2728.2(2)

4

1.254

1.468

1080.0

$0.7 \times 0.4 \times 0.1$

$\operatorname{CuK} \alpha(\lambda=1.54184)$

7.678 to 143.434

$-16 \leq \mathrm{h} \leq 16,-13 \leq \mathrm{k} \leq 11,-22 \leq 1 \leq 20$

26069

$9184\left[R_{\text {int }}=0.0321, R_{\text {sigma }}=0.0277\right]$

9184/105/685

1.030

$\mathrm{R}_{1}=0.0775, \mathrm{wR}_{2}=0.2273$

$\mathrm{R}_{1}=0.0917, \mathrm{wR}_{2}=0.2512$

$0.39 /-0.32$

$0.012(12)$ 


\section{Proposed transition state for the desymmetric $\mathrm{RC}$ reaction}

According to the absolute configuration of chiral product $6 \mathbf{m}$, the transition state for the desymmetric $\mathrm{RC}$ reaction has been proposed. As shown in the following scheme, the thiol catalyst $\mathbf{T 5}$ attacks the bis(enones) from the opposite side of the aryl substitution at the $\delta$-position, giving enolate ion pair intermediate IV with the chiral PTC catalyst C7. With the assistant of H-bonding interaction between the hydroxyl group of $\mathbf{C 7}$ and the carbonyl group of $\mathbf{I V}$, the enolate attacks the enone from $\mathrm{Si}$-face via a chair-like transition state (TS).

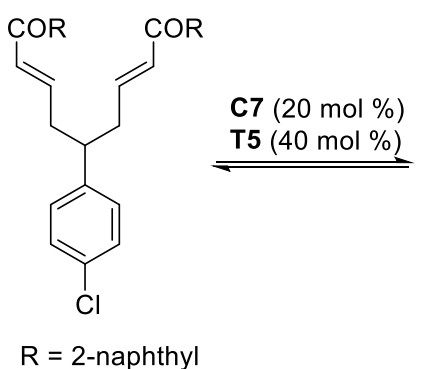

$\mathrm{R}=$ 2-naphthyl

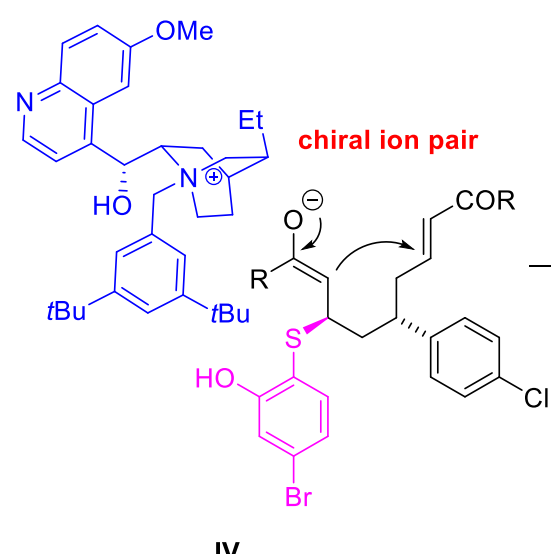

IV

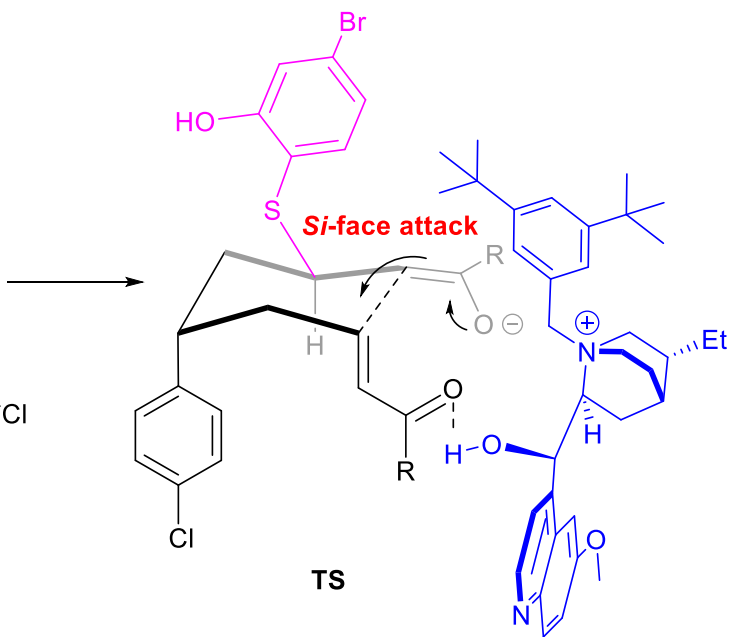

TS

Proposed transition state 


\section{NMR, HRMS spectra and HPLC chromatograms}

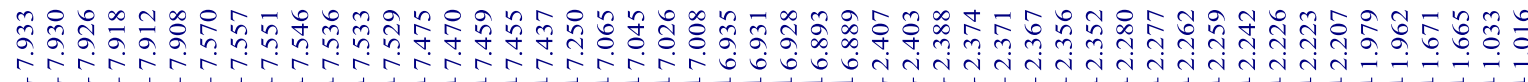<smiles>CC(C/C=C/C(=O)c1ccccc1)C/C=C/C(=O)c1ccccc1</smiles>

$5 \mathbf{a}$

${ }^{1} \mathrm{H}$ NMR $\left(400 \mathrm{MHz}, \mathrm{CDCl}_{3}\right)$

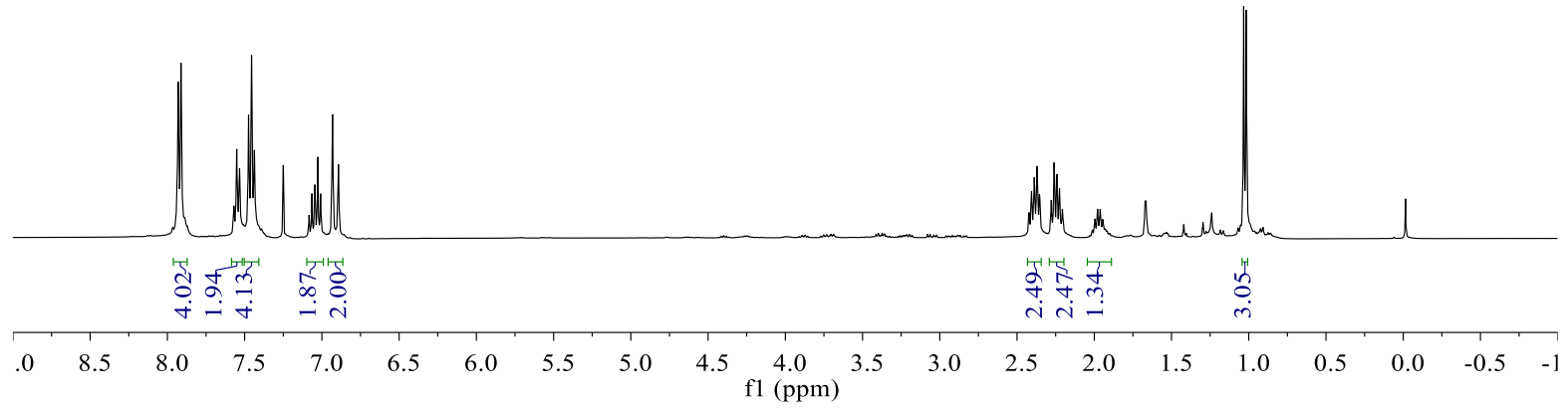

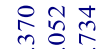

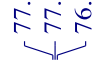<smiles>CC(C/C=C/C(=O)c1ccccc1)C/C=C/C(=O)c1ccccc1</smiles>

$5 a$

${ }^{13} \mathrm{C}$ NMR $\left(100 \mathrm{MHz}, \mathrm{CDCl}_{3}\right)$

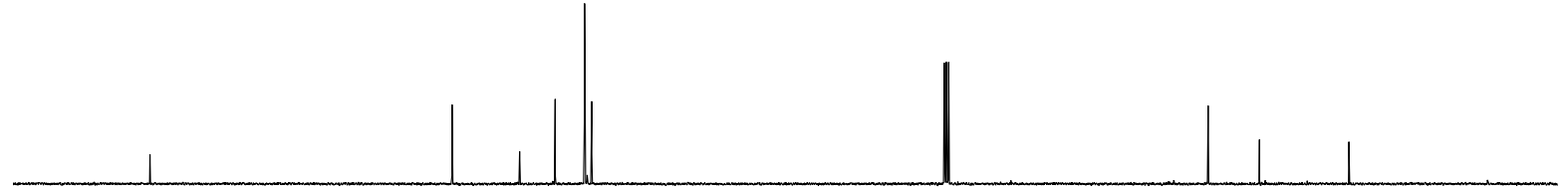

$\begin{array}{llllllllllllllllllllll}10 & 200 & 190 & 180 & 170 & 160 & 150 & 140 & 130 & 120 & 110 & \begin{array}{c}100 \\ \mathrm{f} 1(\mathrm{ppm})\end{array} & 90 & 80 & 70 & 60 & 50 & 40 & 30 & 20 & 10 & 0\end{array}$ 


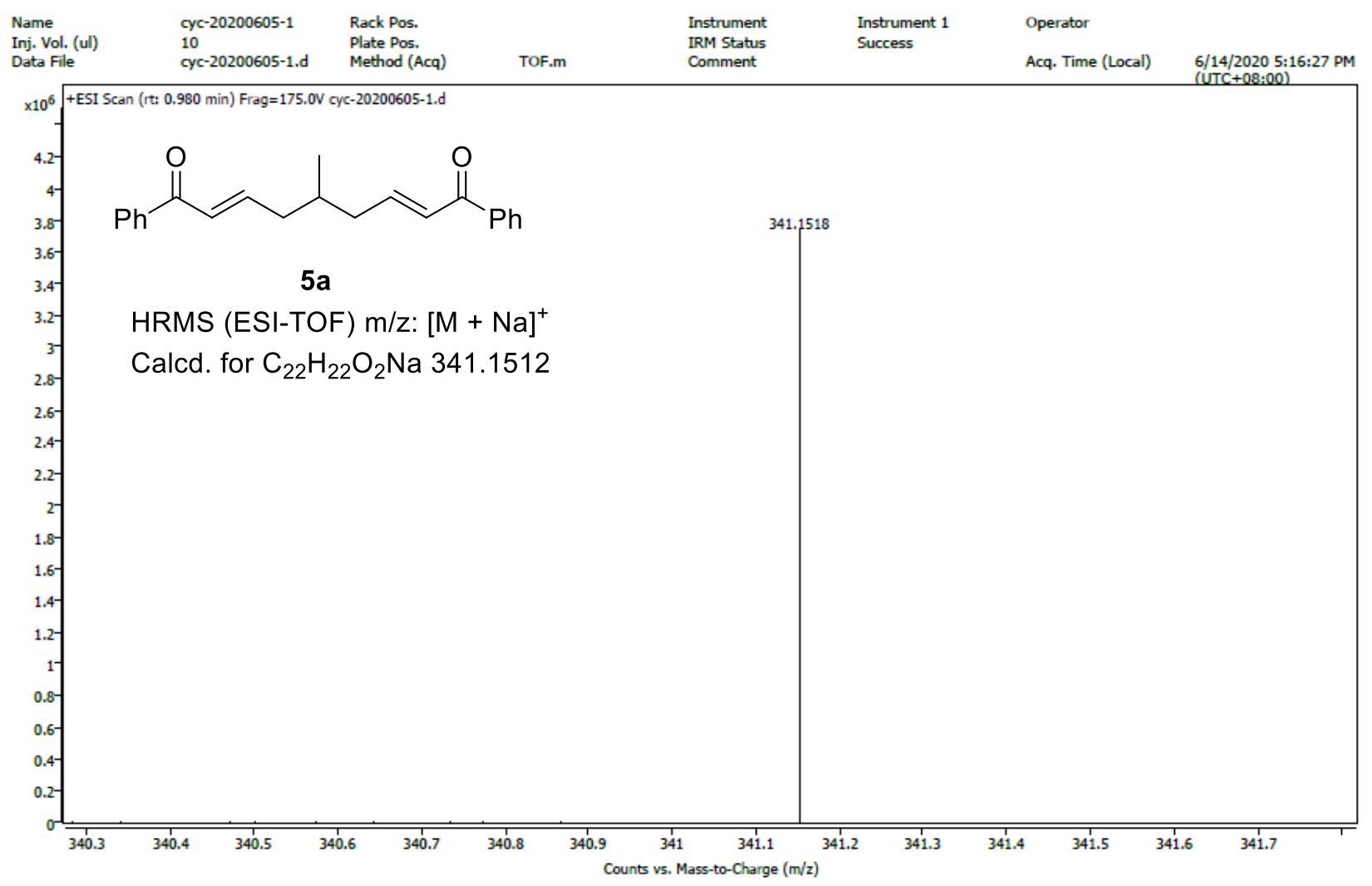


<smiles>Cc1ccc(C(=O)/C=C/CC(C)C/C=C/C(=O)c2ccc(C)cc2)cc1</smiles>

5b

${ }^{1} \mathrm{H}$ NMR $\left(400 \mathrm{MHz}, \mathrm{CDCl}_{3}\right)$

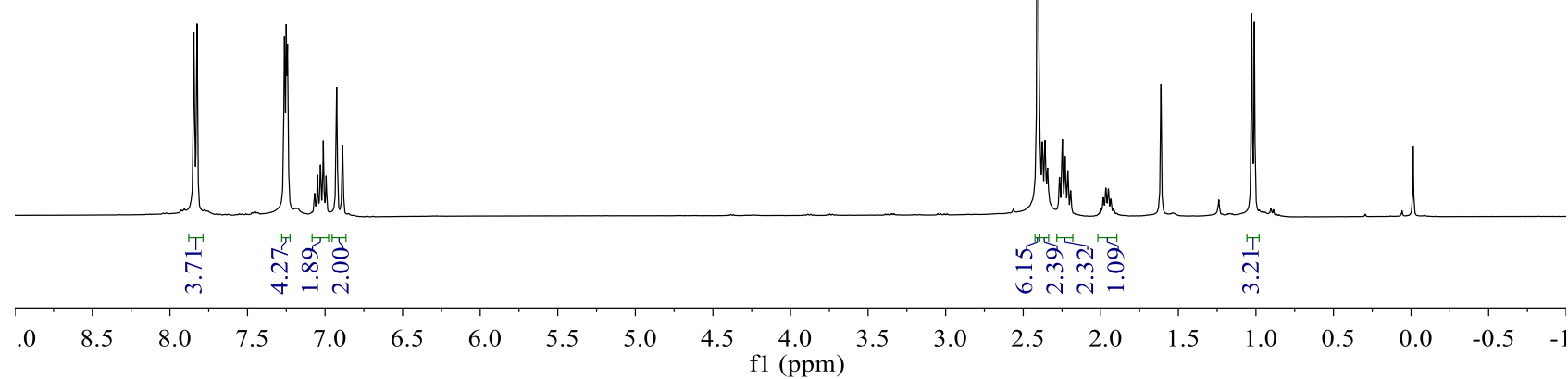<smiles>Cc1ccc(C(=O)/C=C/CC(C)C/C=C/C(=O)c2ccc(C)cc2)cc1</smiles>

$5 b$

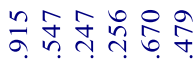

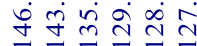

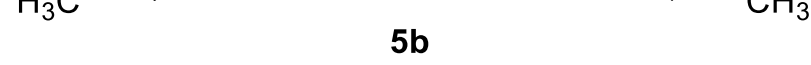

${ }^{13} \mathrm{C}$ NMR $\left(150 \mathrm{MHz}, \mathrm{CDCl}_{3}\right)$

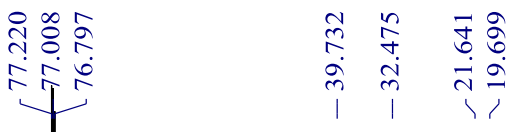




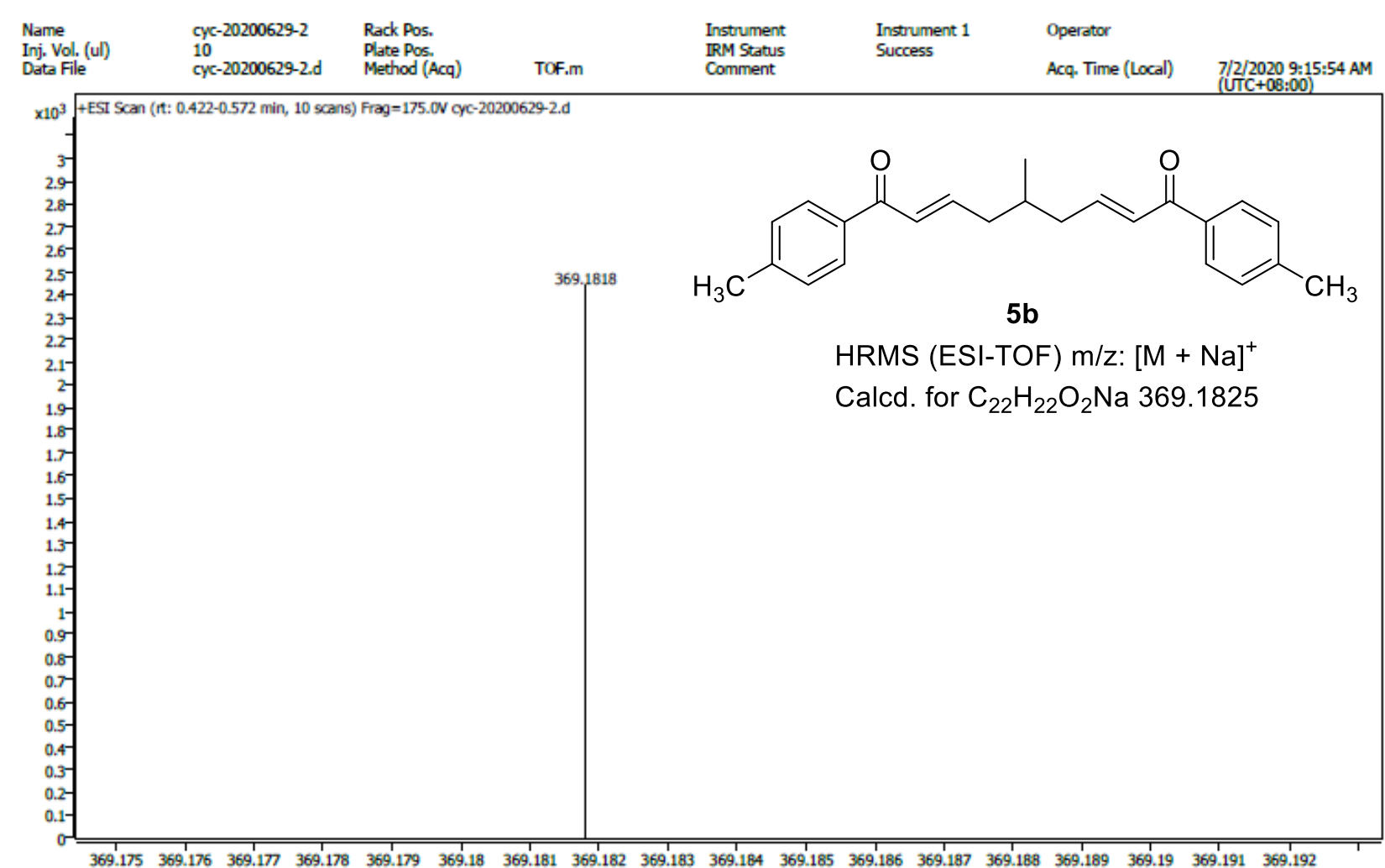


<smiles>COc1cccc(C(=O)/C=C/CC(C)C/C=C/C(=O)c2cccc(OC)c2)c1</smiles>

$5 c$

${ }^{1} \mathrm{H}$ NMR $\left(400 \mathrm{MHz}, \mathrm{CDCl}_{3}\right)$

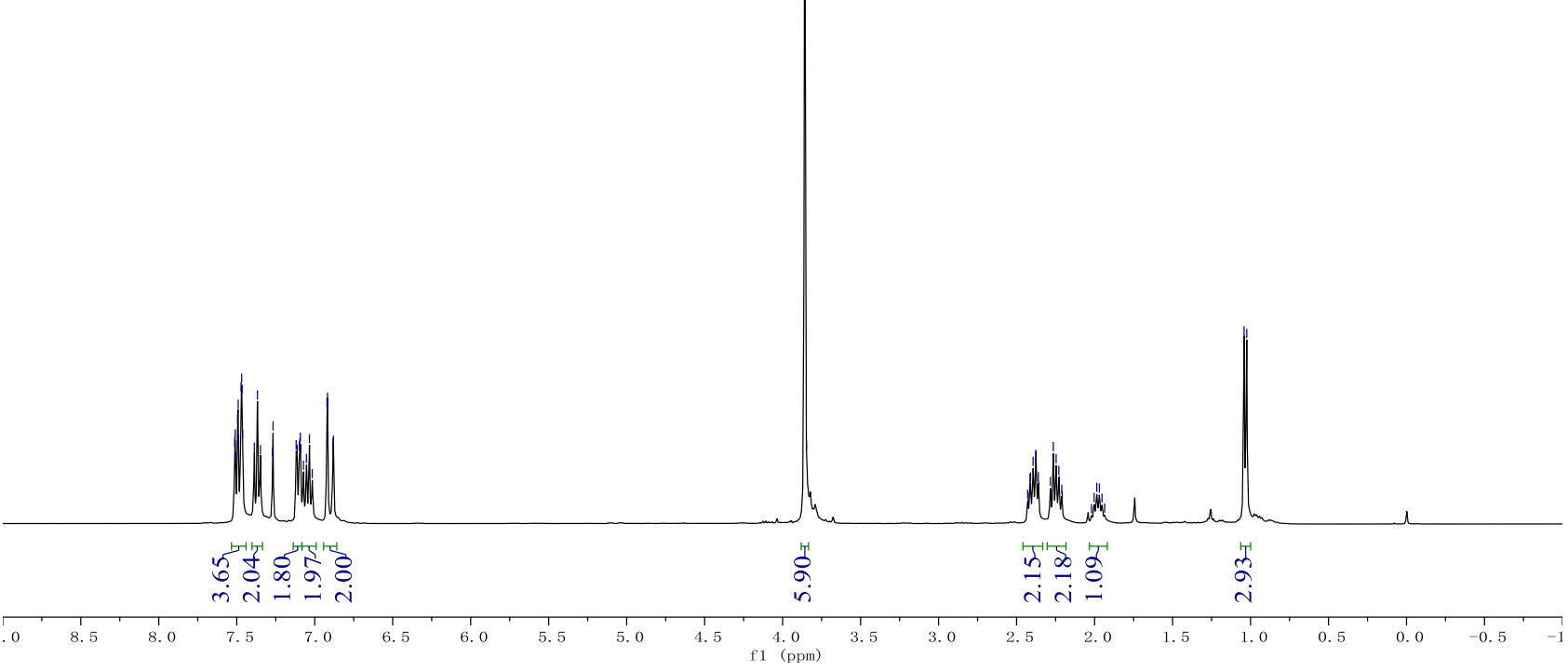

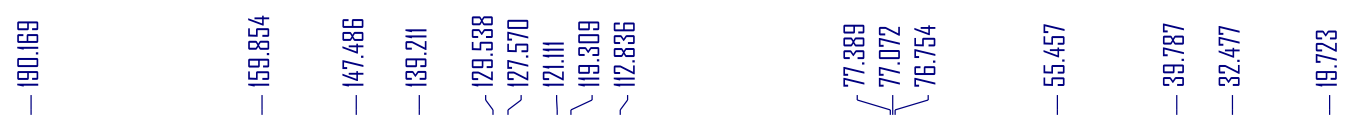<smiles>COc1cccc(C(=O)/C=C/CC(C)C/C=C/C(=O)c2cccc(OC)c2)c1</smiles>

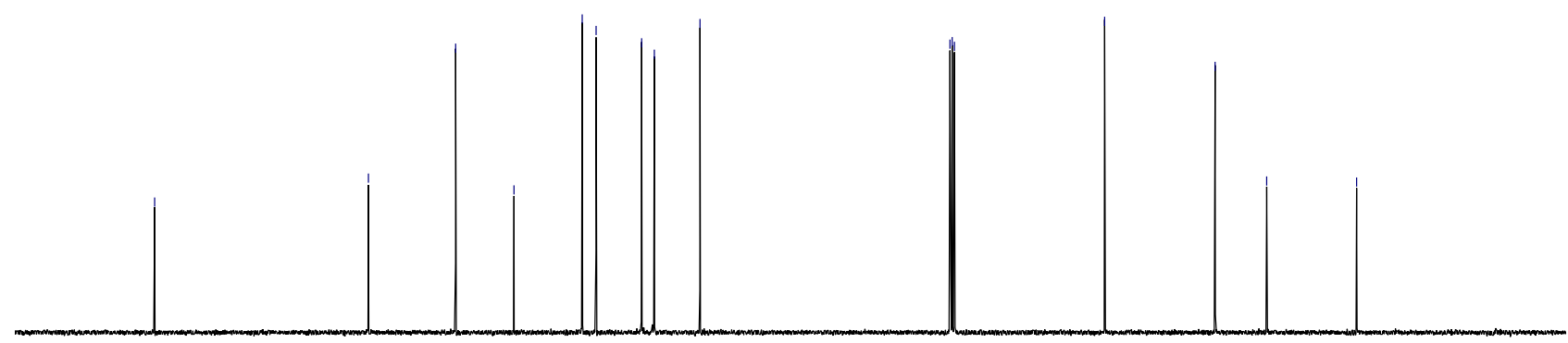

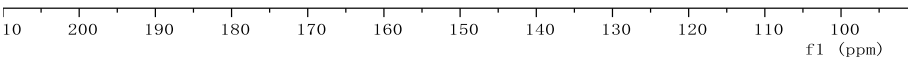




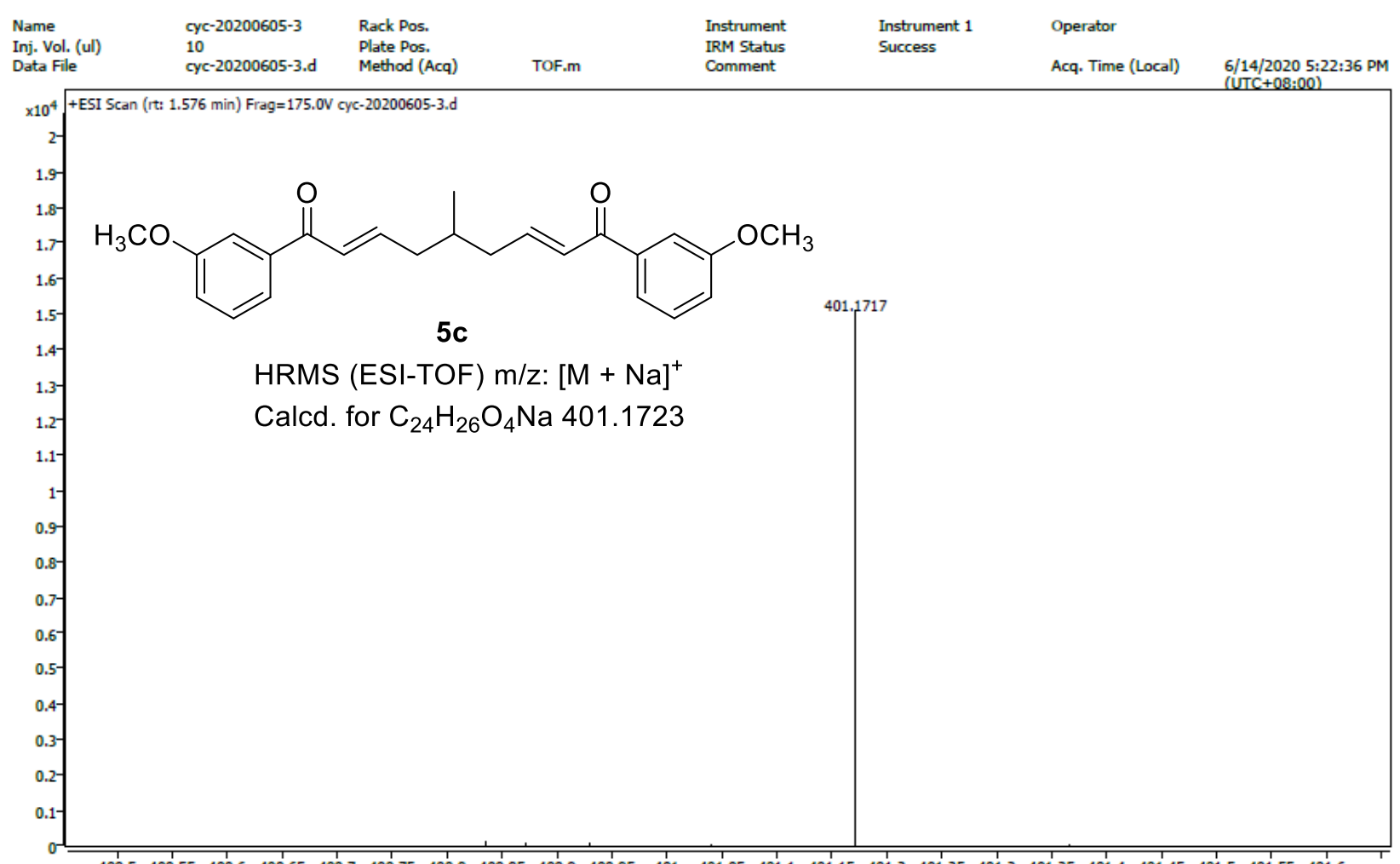

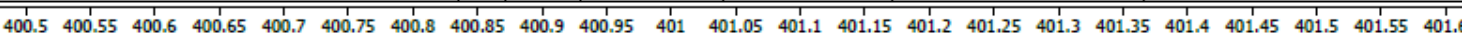
Counts vs. Mass-to-Charge (m/z) 


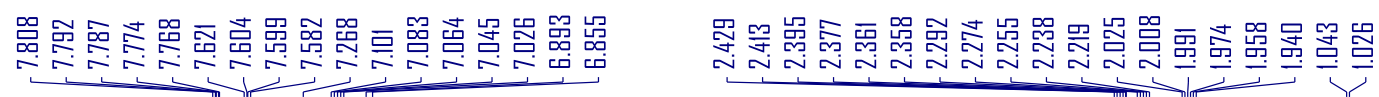<smiles>CC(C/C=C/C(=O)c1ccc(Br)cc1)C/C=C/C(=O)c1ccc(Br)cc1</smiles>

5d

${ }^{1} \mathrm{H}$ NMR $\left(400 \mathrm{MHz}, \mathrm{CDCl}_{3}\right)$

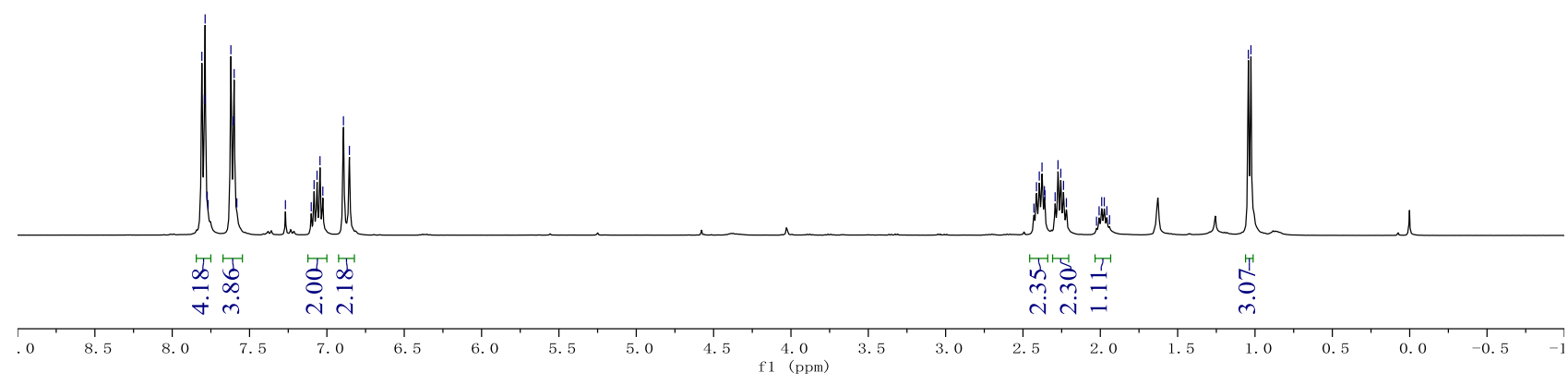

\begin{tabular}{|c|c|c|c|}
\hline $\begin{array}{l}\text { 骂 } \\
\text { 咱 }\end{array}$ & 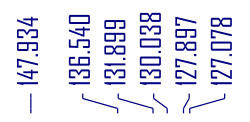 & 喜总点 & \\
\hline
\end{tabular}<smiles>CC(C/C=C/C(=O)c1ccc(Br)cc1)C/C=C/C(=O)c1ccc(Br)cc1</smiles>

5d

${ }^{13} \mathrm{C}$ NMR $\left(100 \mathrm{MHz}, \mathrm{CDCl}_{3}\right)$

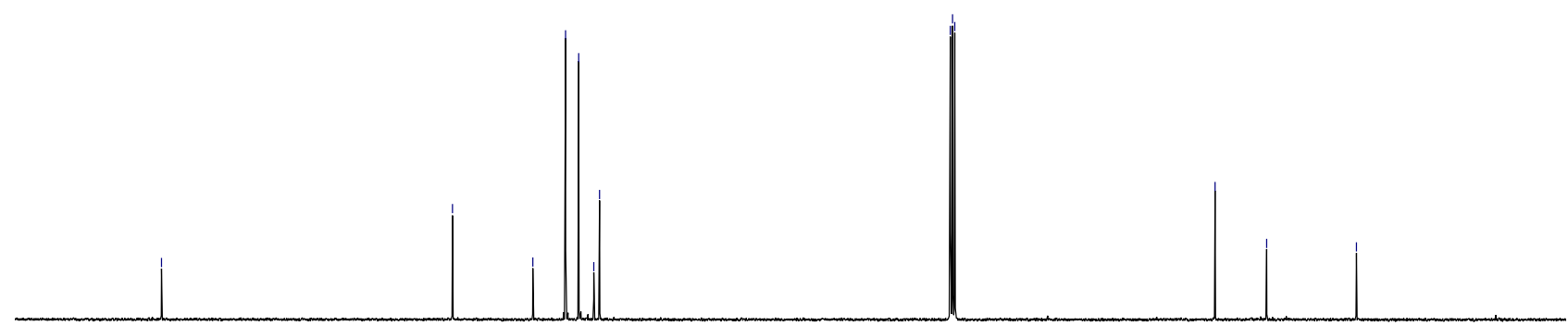

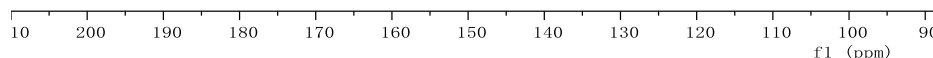


<smiles>CC(C/C=C/C(=O)c1ccc(Br)cc1)C/C=C/C(=O)c1ccc(Br)cc1</smiles>

5d

HRMS (ESI-TOF) m/z: [M + Na]

Calcd. for $\mathrm{C}_{22} \mathrm{H}_{20} \mathrm{Br}^{79} \mathrm{Br}^{81} \mathrm{O}_{2} \mathrm{Na} 498.9702$

Calcd. for $\mathrm{C}_{22} \mathrm{H}_{20} \mathrm{Br}^{79}{ }_{2} \mathrm{O}_{2} \mathrm{Na} 496.9722$

Calcd. for $\mathrm{C}_{22} \mathrm{H}_{20} \mathrm{Br}^{81}{ }_{2} \mathrm{O}_{2} \mathrm{Na} 500.9681$
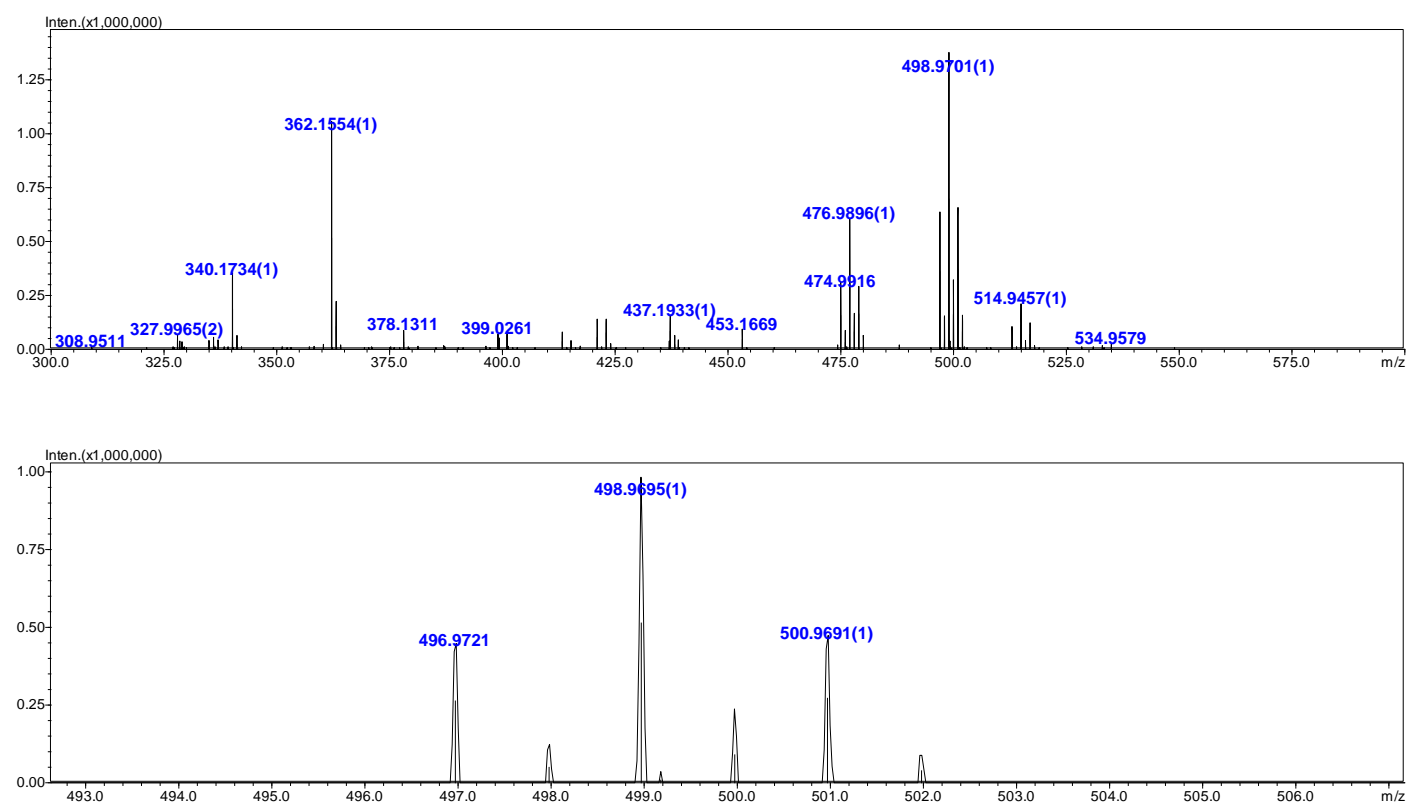


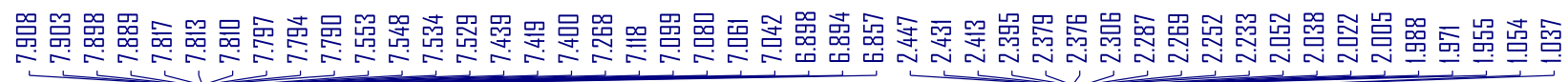<smiles>CC(C/C=C/C(=O)c1cccc(Cl)c1)C/C=C/C(=O)c1cccc(Cl)c1</smiles>

$5 e$

${ }^{1} \mathrm{H} \mathrm{NMR}\left(400 \mathrm{MHz}, \mathrm{CDCl}_{3}\right)$
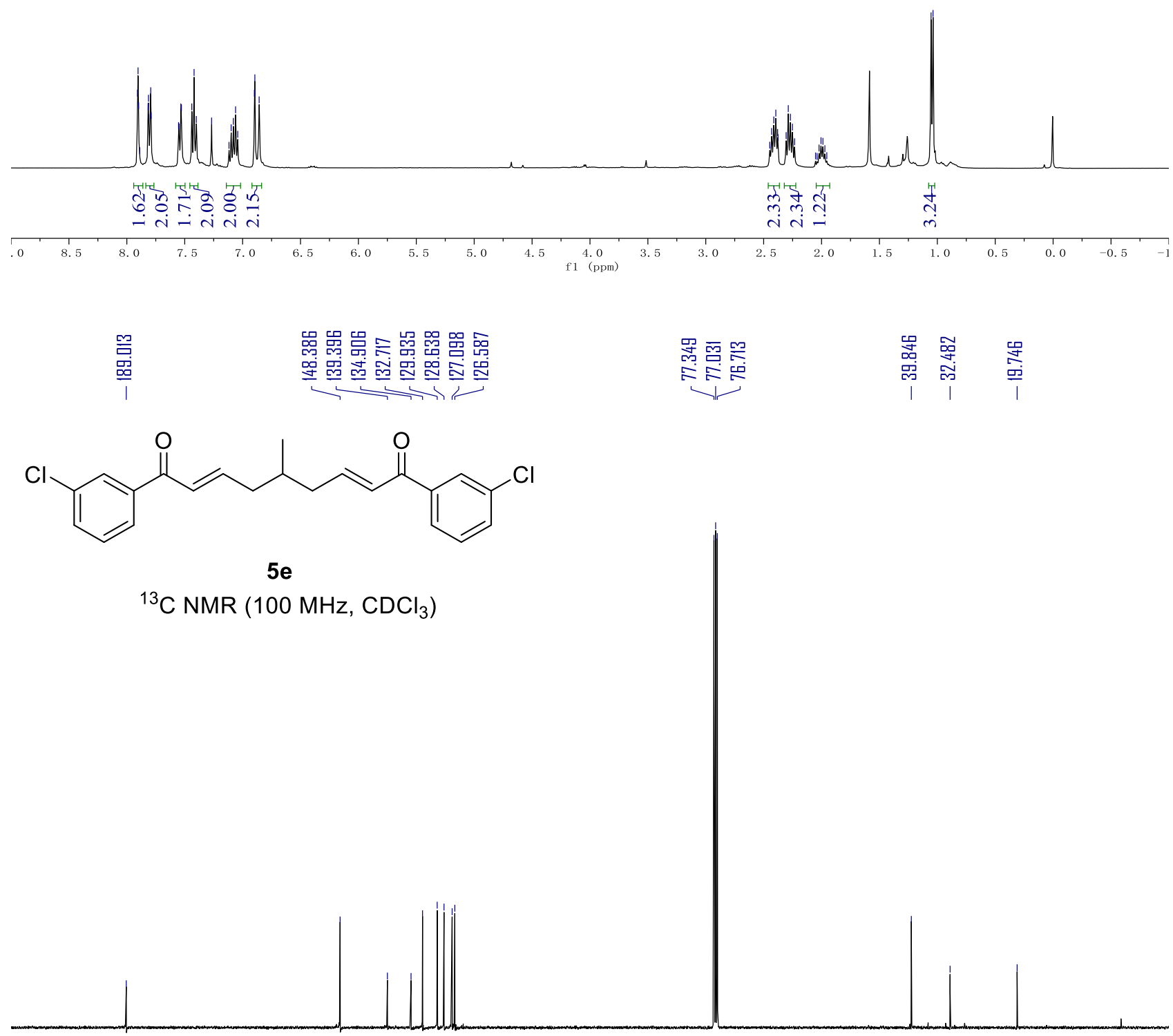

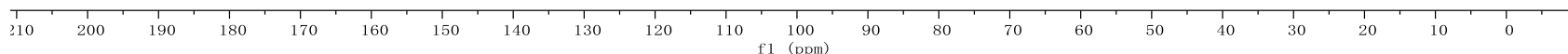


<smiles>CC(C/C=C/C(=O)c1cccc(Cl)c1)C/C=C/C(=O)c1cccc(Cl)c1</smiles>

HRMS (ESI-TOF) m/z: [M + Na]

Calcd. for $\mathrm{C}_{22} \mathrm{H}_{20} \mathrm{Cl}^{35}{ }_{2} \mathrm{O}_{2} \mathrm{Na} 409.0733$

Calcd. for $\mathrm{C}_{22} \mathrm{H}_{20} \mathrm{Cl}^{35} \mathrm{Cl}^{37} \mathrm{O}_{2} \mathrm{Na} 411.0703$
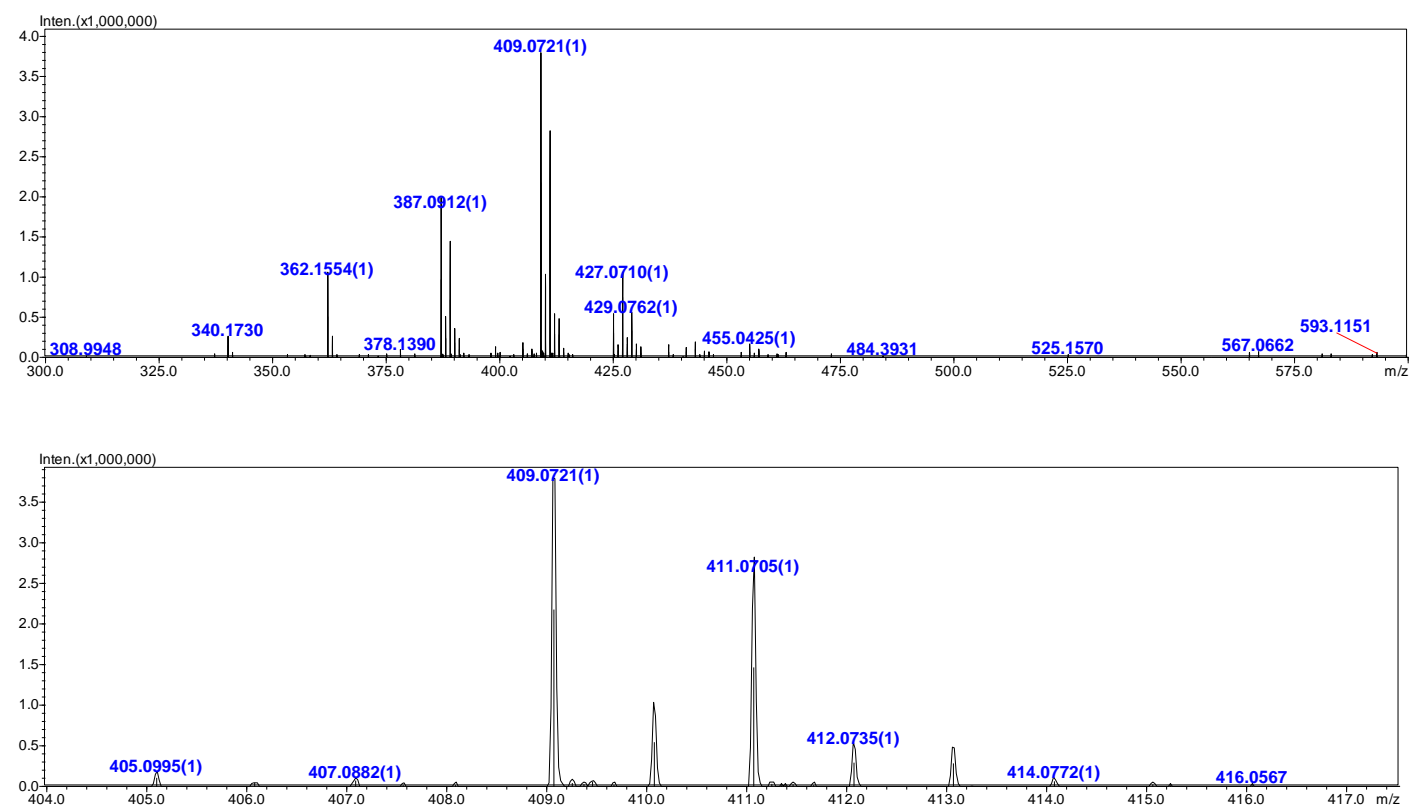
<smiles>CC(C/C=C/C(=O)c1ccc2ccccc2c1)C/C=C/C(=O)c1ccc2ccccc2c1</smiles>

${ }^{1} \mathrm{H}$ NMR $\left(400 \mathrm{MHz}, \mathrm{CDCl}_{3}\right)$
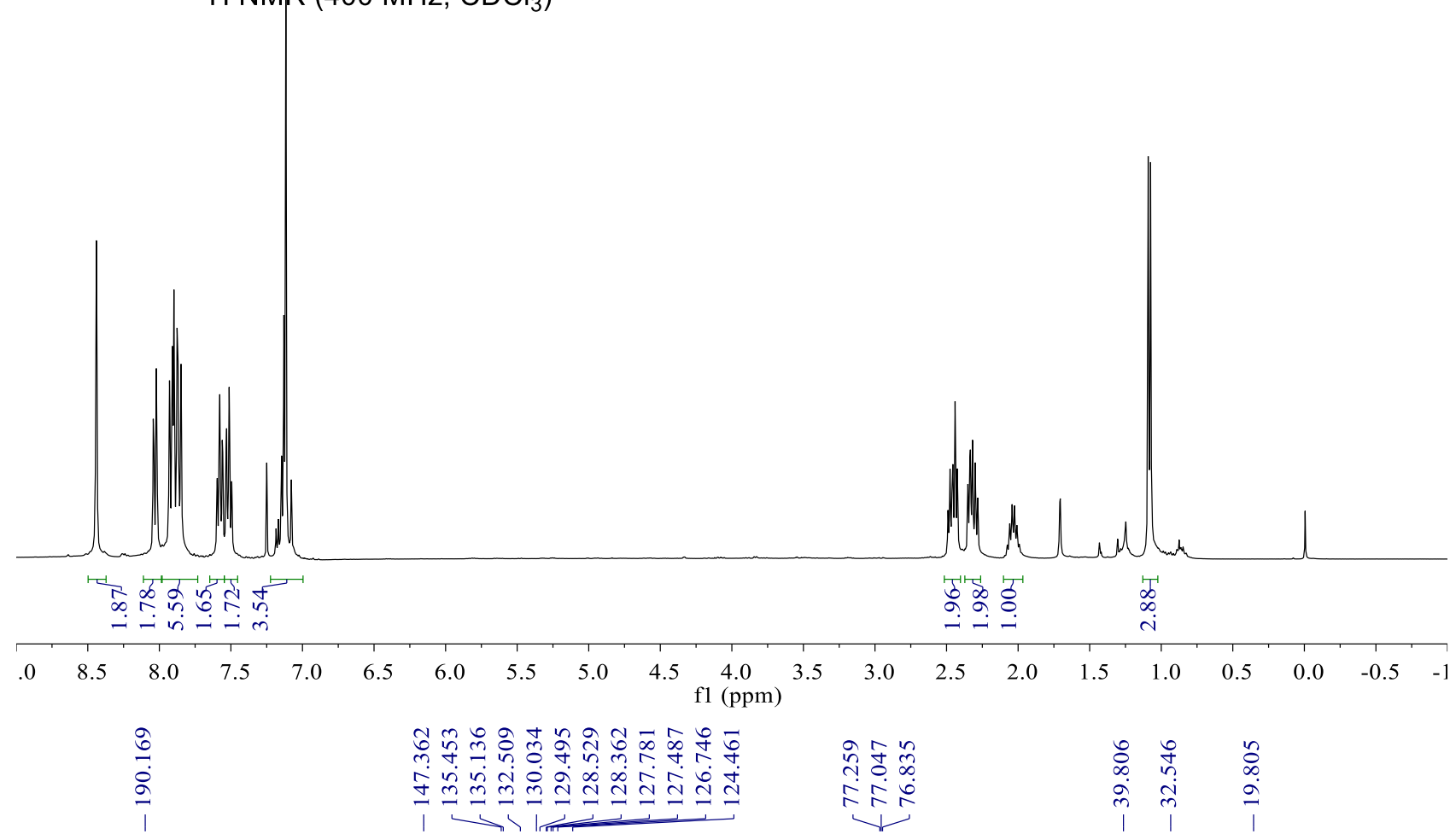

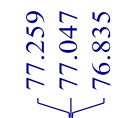

$\begin{array}{lll}0 & 0 & \\ 0 & 0 & 0 \\ 0 & 0 & 0 \\ 0 & 0 & 0 \\ 0 & 0 & 0 \\ 1 & 1 & 1\end{array}$<smiles>CC(C/C=C/C(=O)c1ccc2ccccc2c1)C/C=C/C(=O)c1ccc2ccccc2c1</smiles>

${ }^{13} \mathrm{C}$ NMR $\left(150 \mathrm{MHz}, \mathrm{CDCl}_{3}\right)$

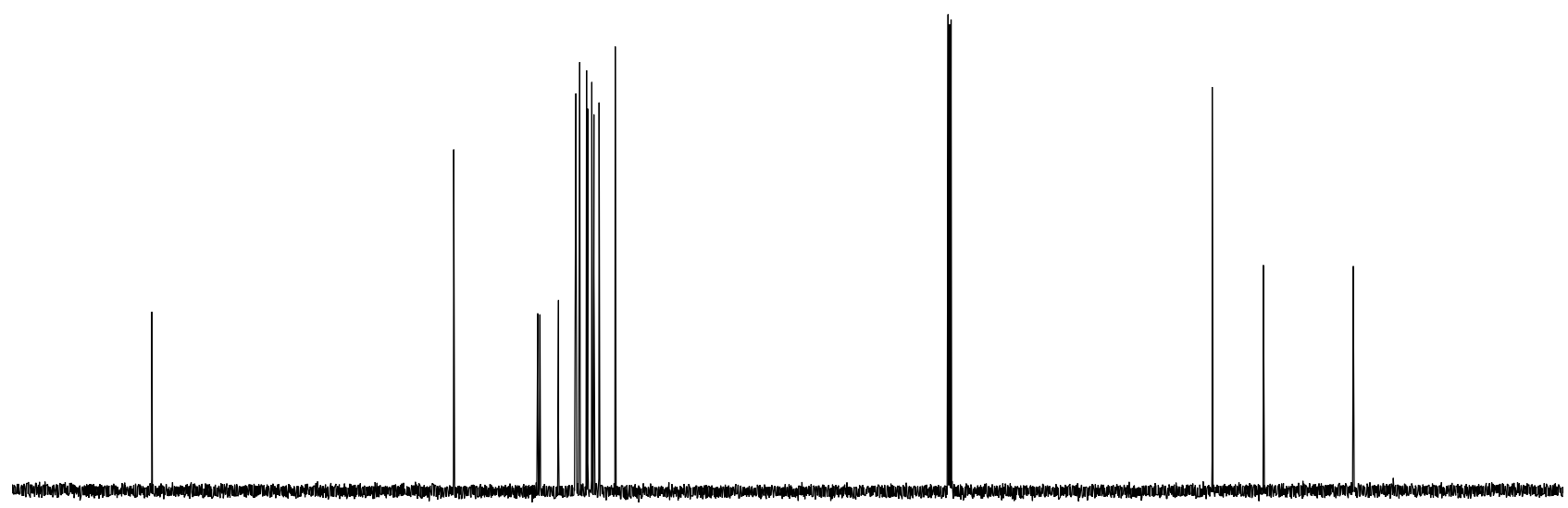

$\begin{array}{llllllllllllllllllllll}200 & 190 & 180 & 170 & 160 & 150 & 140 & 130 & 120 & 110 \begin{array}{c}100 \\ \mathrm{f} 1(\mathrm{ppm})\end{array} & 90 & 80 & 70 & 60 & 50 & 40 & 30 & 20 & 10 & 0 & -\end{array}$ 
<smiles>CC(C/C=C/C(=O)c1ccc2ccccc2c1)C/C=C/C(=O)c1ccc2ccccc2c1</smiles>

$5 f$

HRMS (ESI-TOF) m/z: [M + Na] ${ }^{+}$

Calcd. for $\mathrm{C}_{30} \mathrm{H}_{26} \mathrm{O}_{2} \mathrm{Na} 441.1825$
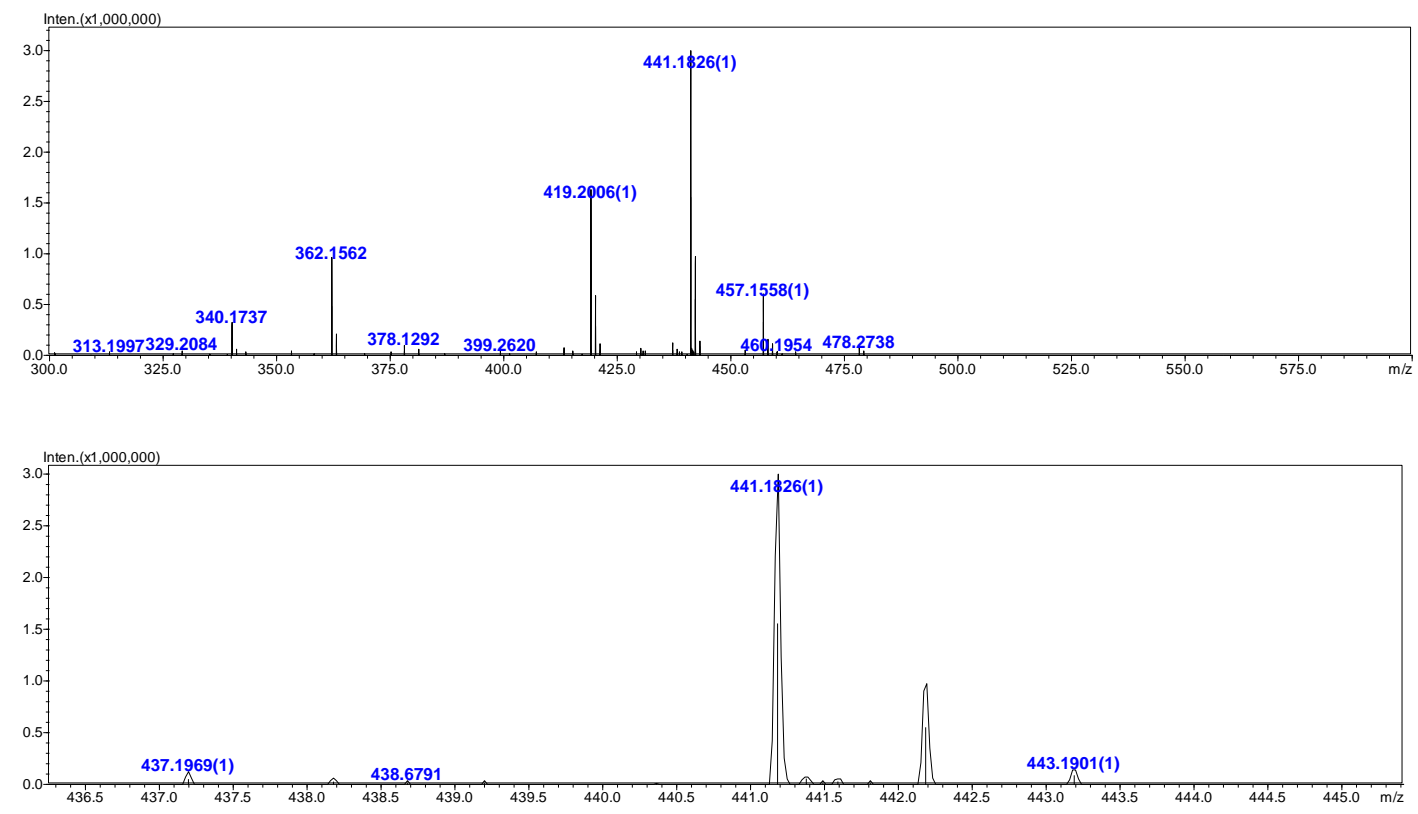
<smiles>CC(C/C=C/C(=O)c1cccs1)C/C=C/C(=O)c1cccs1</smiles>

${ }^{1} \mathrm{H}$ NMR $\left(400 \mathrm{MHz}, \mathrm{CDCl}_{3}\right)$

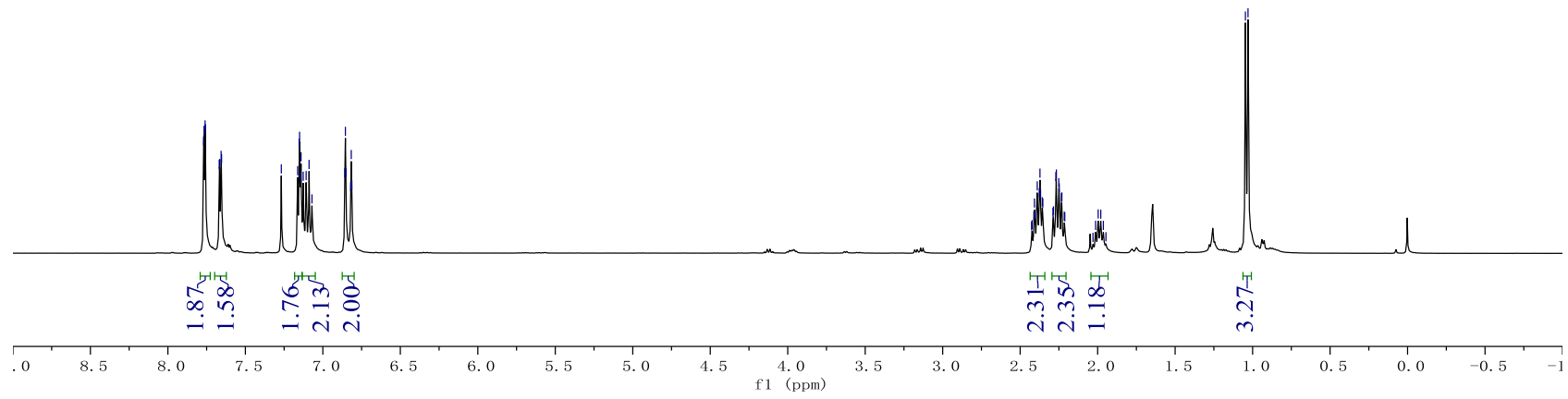

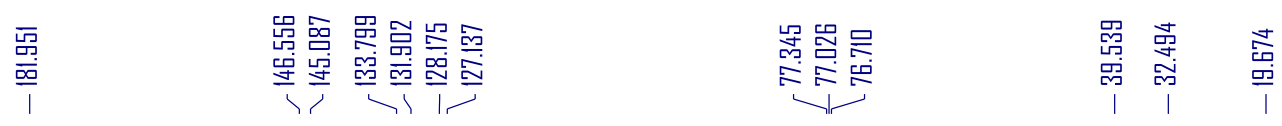

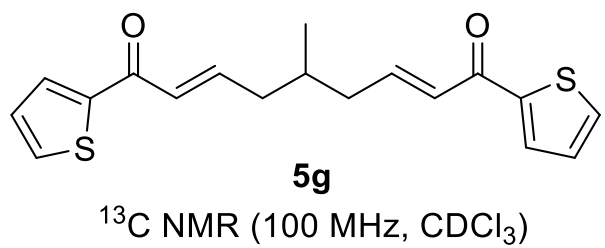

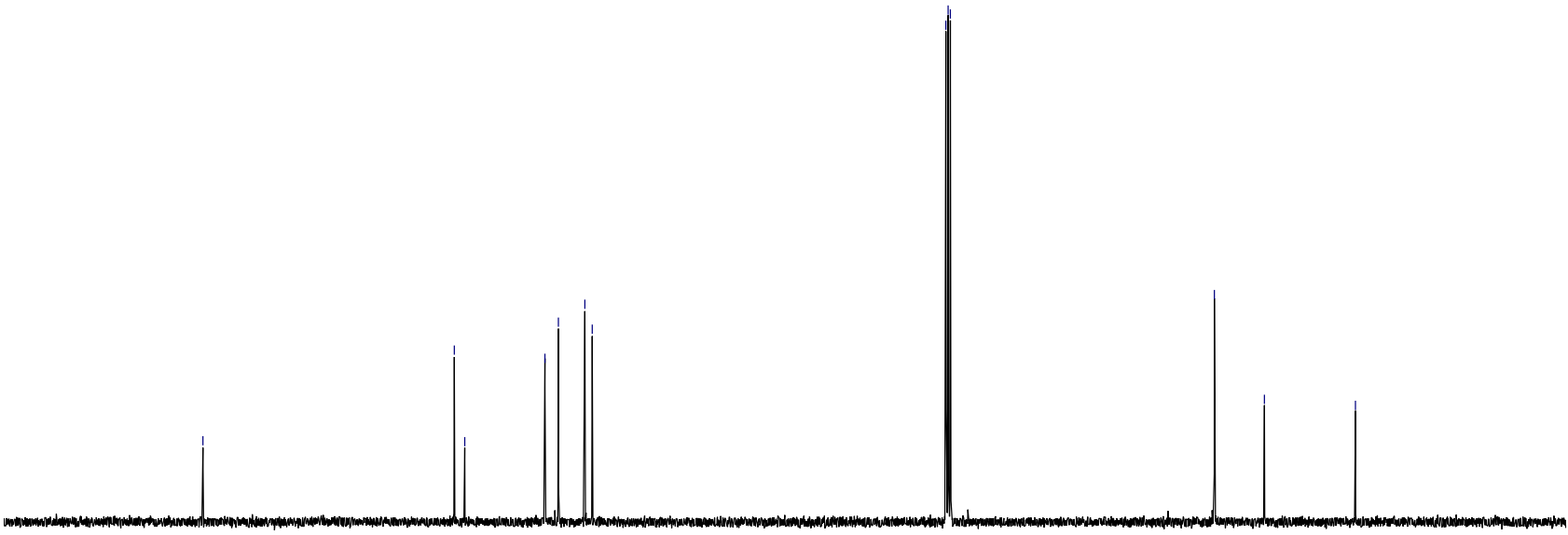

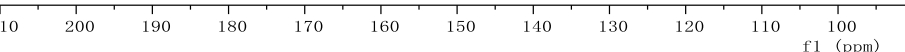




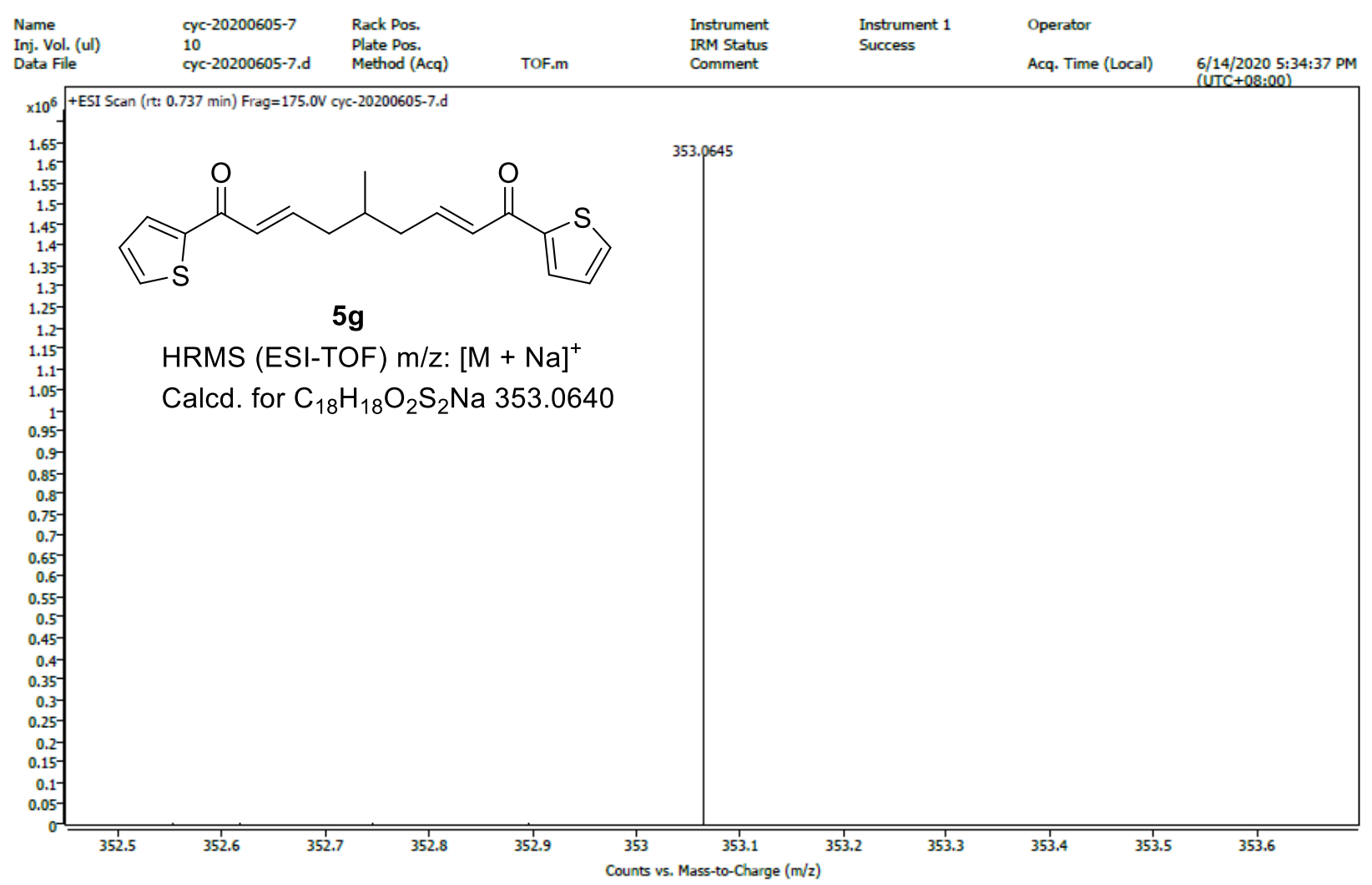


<smiles>O=C(/C=C/CC(C/C=C/C(=O)c1ccccc1)c1ccccc1)c1ccccc1</smiles>

$5 \mathrm{~h}$

${ }^{1} \mathrm{H}$ NMR $\left(400 \mathrm{MHz}, \mathrm{CDCl}_{3}\right)$

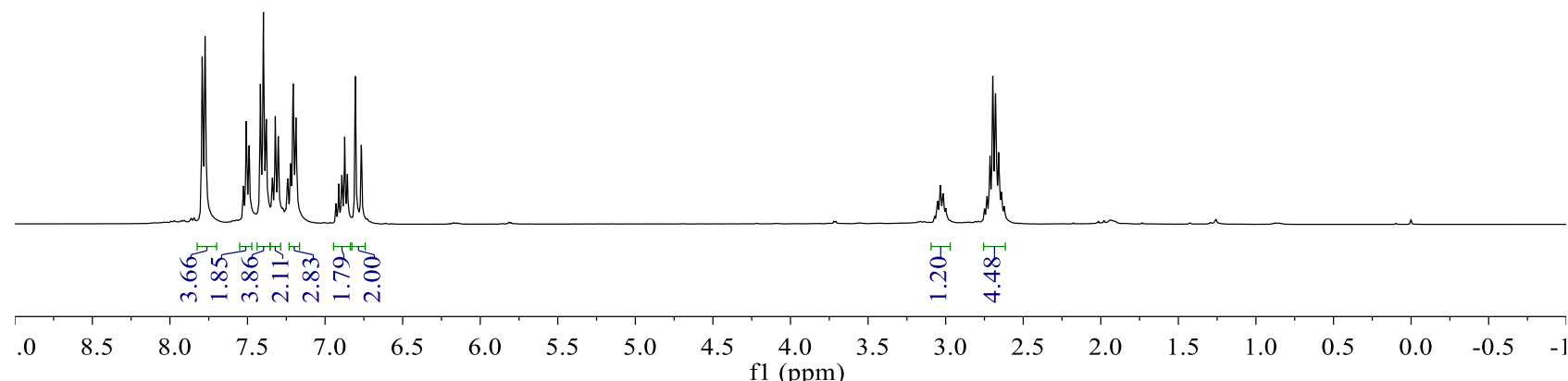

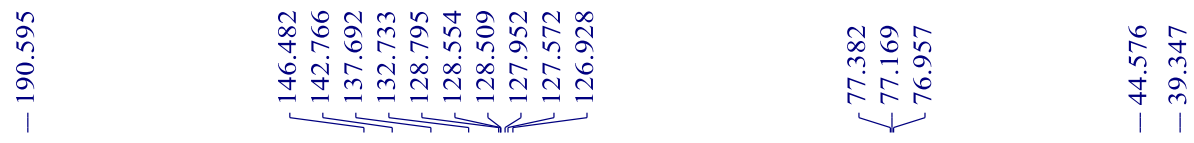<smiles>O=C(/C=C/CC(C/C=C/C(=O)c1ccccc1)c1ccccc1)c1ccccc1</smiles>

$5 \mathrm{~h}$

${ }^{13} \mathrm{C} \mathrm{NMR}\left(150 \mathrm{MHz}, \mathrm{CDCl}_{3}\right)$

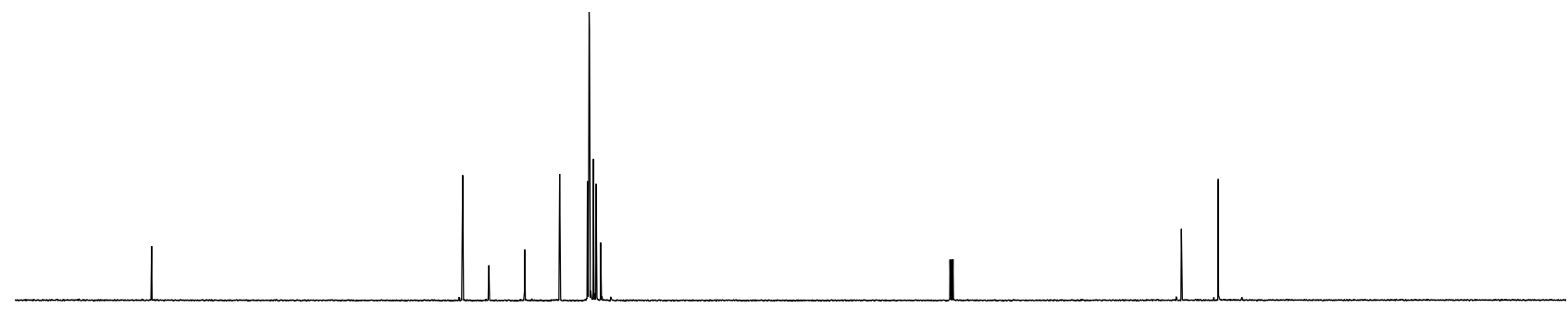

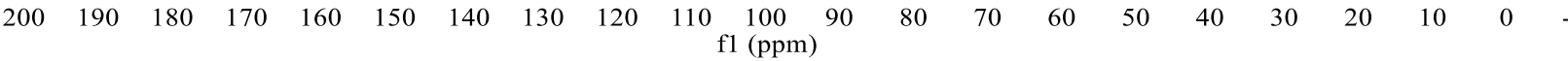




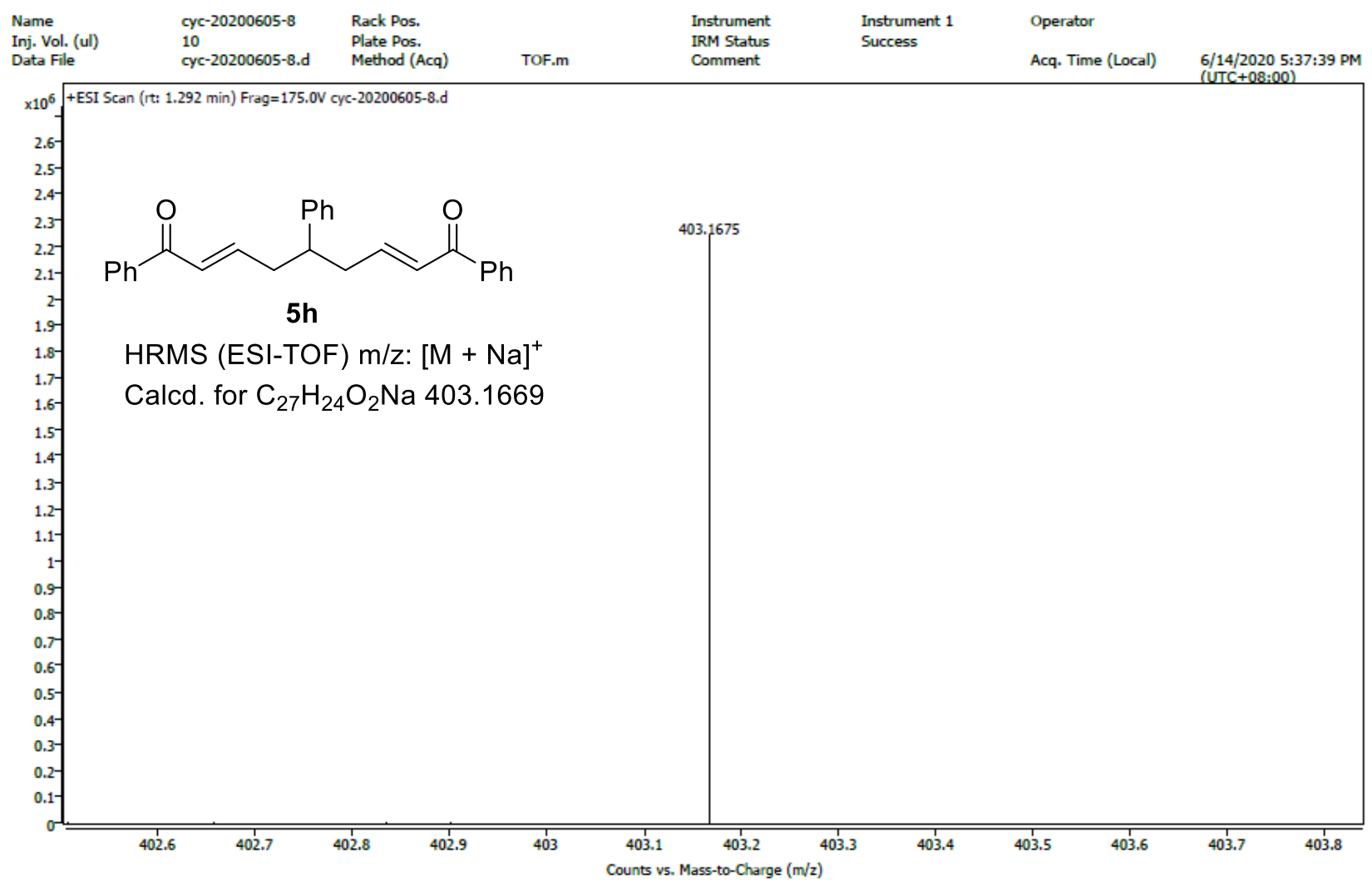


<smiles>O=C(/C=C/CC(C/C=C/C(=O)c1ccccc1)c1ccc(Cl)cc1)c1ccccc1</smiles>

$5 \mathbf{i}$

${ }^{1} \mathrm{H}$ NMR $\left(400 \mathrm{MHz}, \mathrm{CDCl}_{3}\right)$

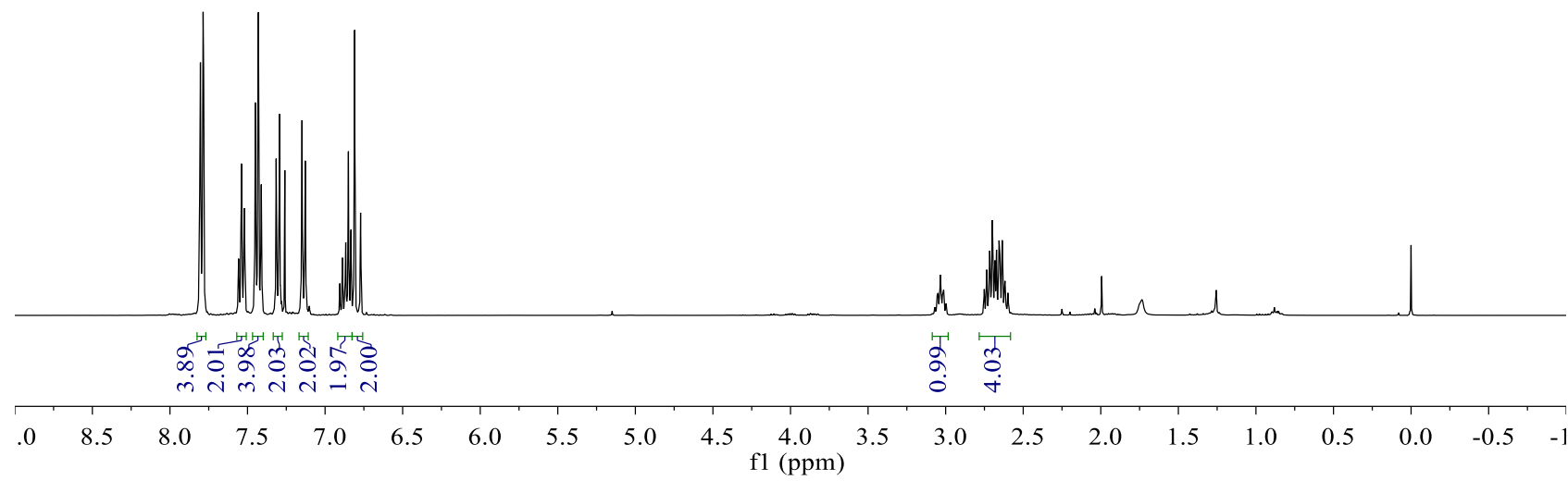

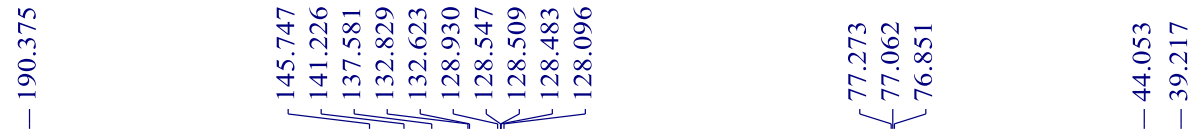<smiles>O=C(/C=C/CC(C/C=C/C(=O)c1ccccc1)c1ccc(Cl)cc1)c1ccccc1</smiles>

$5 \mathbf{i}$

${ }^{13} \mathrm{C}$ NMR $\left(150 \mathrm{MHz}, \mathrm{CDCl}_{3}\right)$

$\begin{array}{lllllllllllllllllllllllll}10 & 200 & 190 & 180 & 170 & 160 & 150 & 140 & 130 & 120 & 110 & 100 & 90 & 80 & 70 & 60 & 50 & 40 & 30 & 20 & 10 & 0 & -\end{array}$ 


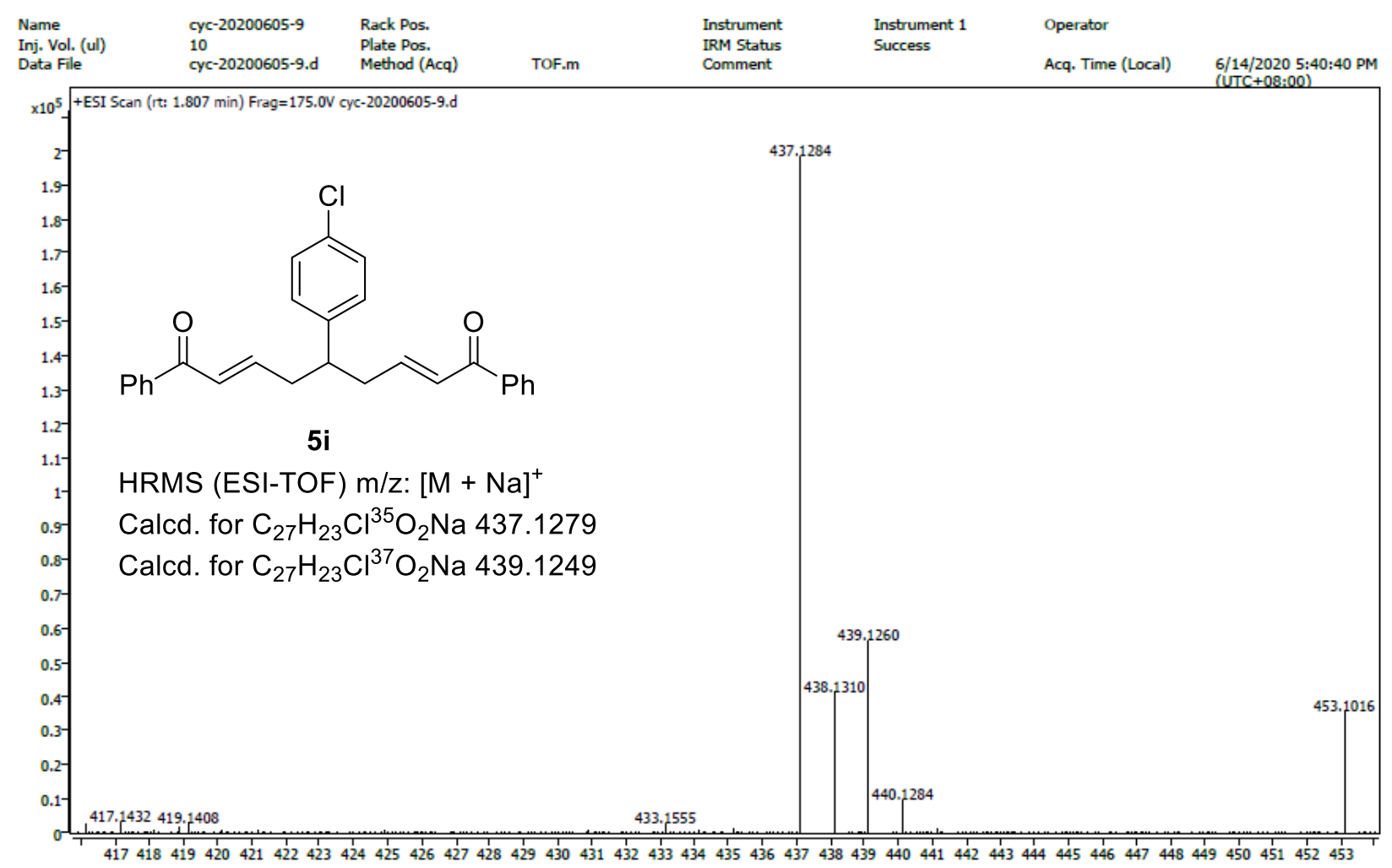
Counts vs. Mass-to-Charge $(\mathrm{m} / \mathrm{z})$ 
<smiles>CCOC(=O)C(C/C=C/C(=O)c1ccccc1)C/C=C/C(=O)c1ccccc1</smiles>

$5 \mathbf{j}$

${ }^{1} \mathrm{H}$ NMR $\left(600 \mathrm{MHz}, \mathrm{CDCl}_{3}\right)$

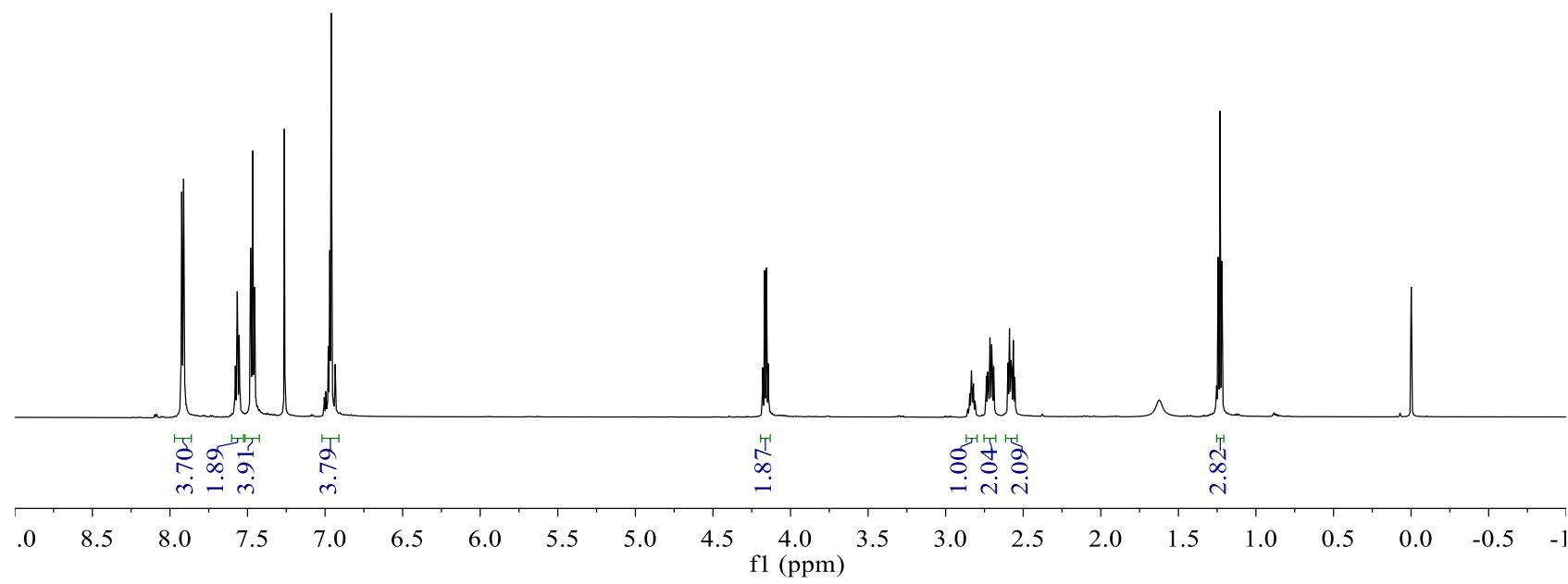<smiles>CCOC(=O)C(C/C=C/C(=O)c1ccccc1)C/C=C/C(=O)c1ccccc1</smiles>

$5 \mathbf{j}$

${ }^{13} \mathrm{C}$ NMR $\left(150 \mathrm{MHz}, \mathrm{CDCl}_{3}\right)$
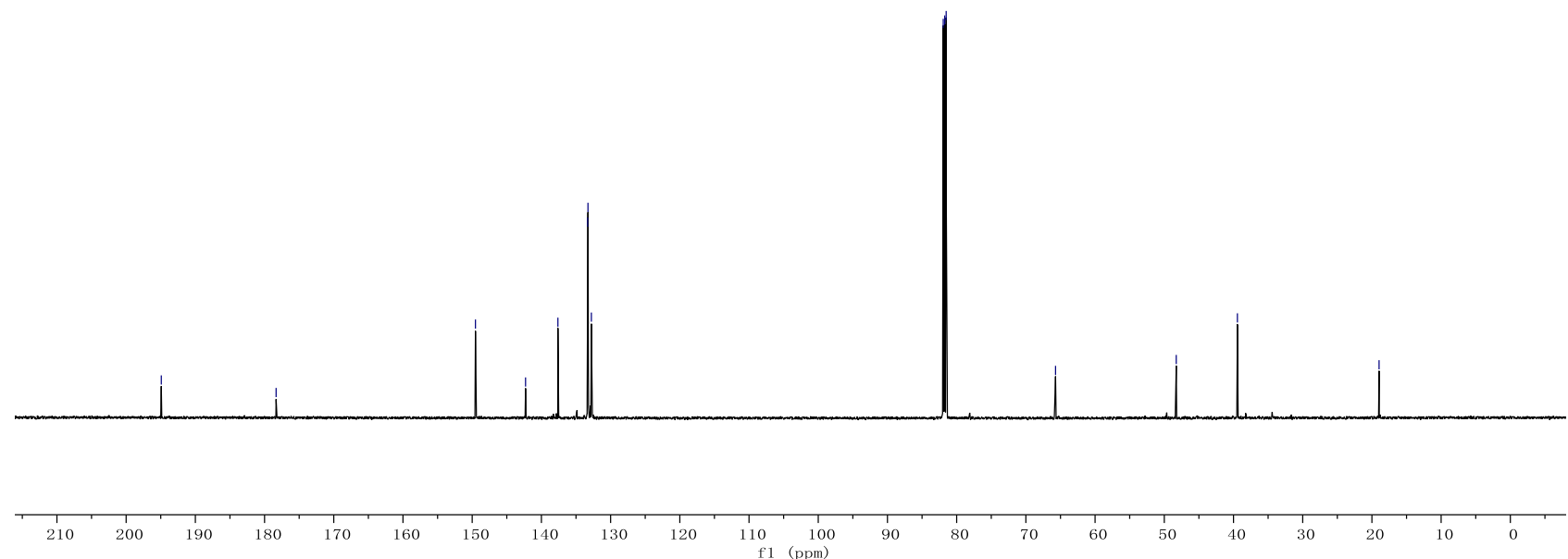


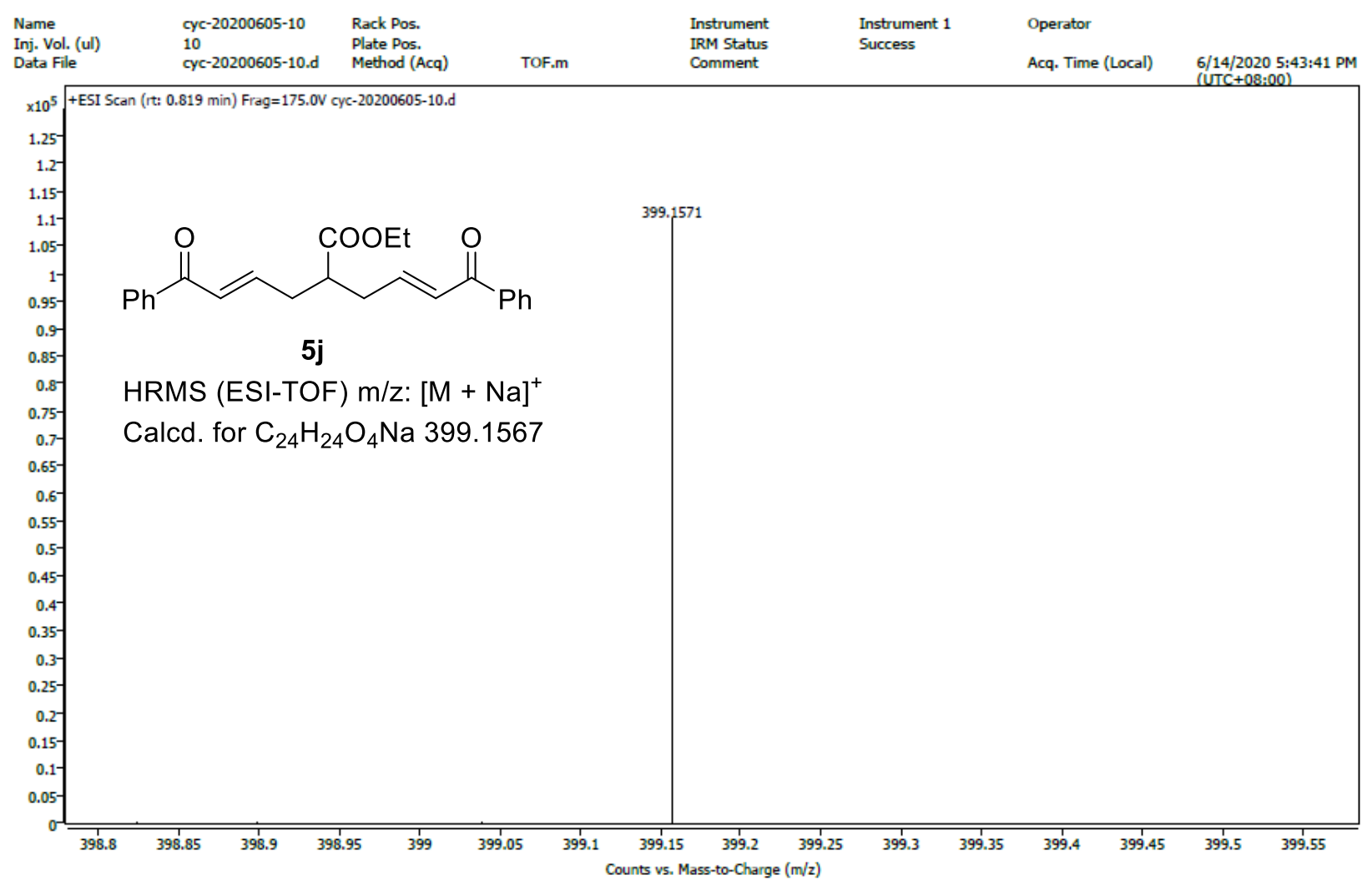


<smiles>CC(C)CC(C/C=C/C(=O)c1ccccc1)C/C=C/C(=O)c1ccccc1</smiles>

5 I

${ }^{1} \mathrm{H} \mathrm{NMR}\left(400 \mathrm{MHz}, \mathrm{CDCl}_{3}\right)$

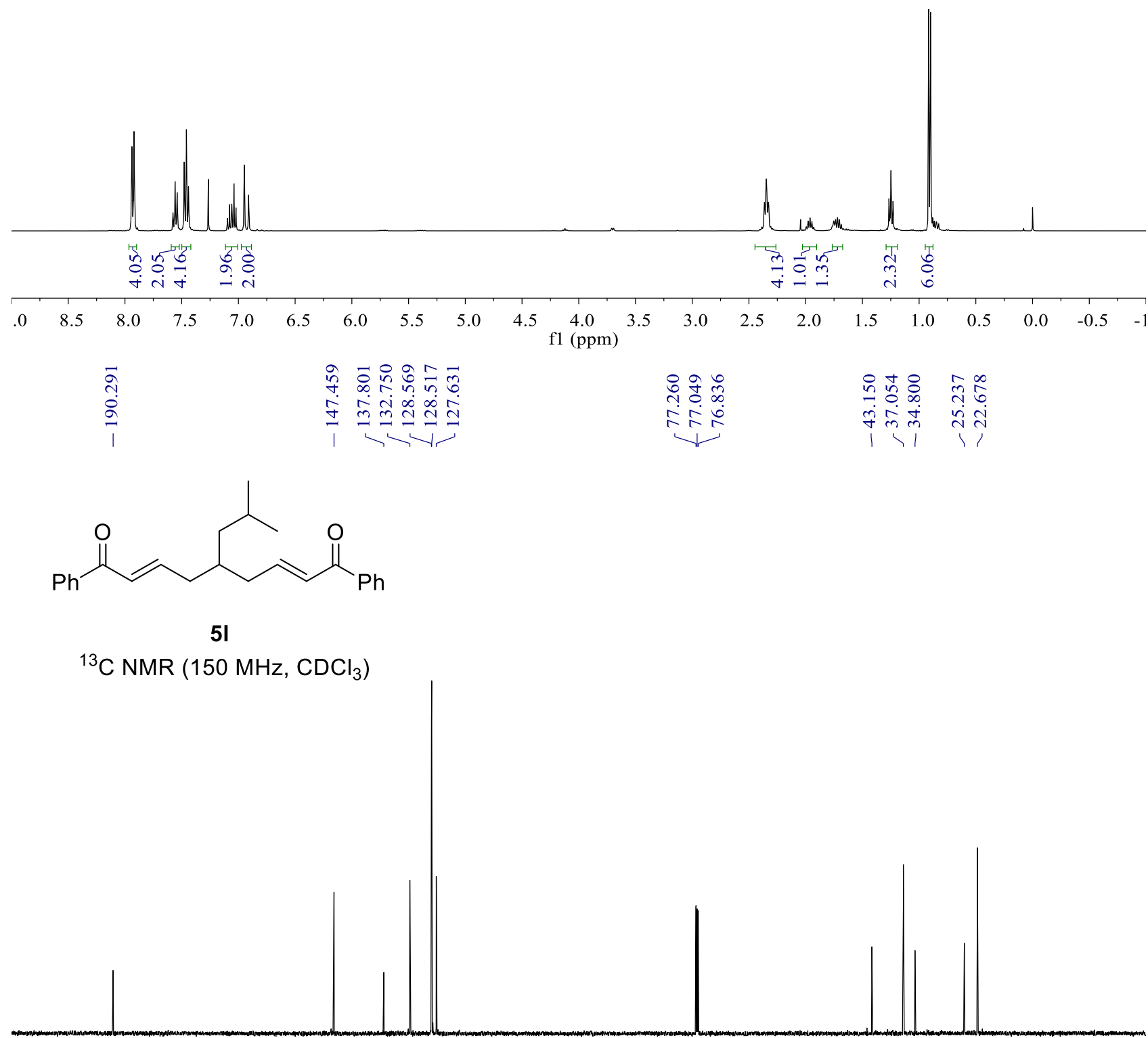

$\begin{array}{llllllllllllllllllllll}200 & 190 & 180 & 170 & 160 & 150 & 140 & 130 & 120 & 110 \underset{\mathrm{f} 1 \mathrm{(ppm})}{100} & 90 & 80 & 70 & 60 & 50 & 40 & 30 & 20 & 10 & 0 & -\end{array}$ 


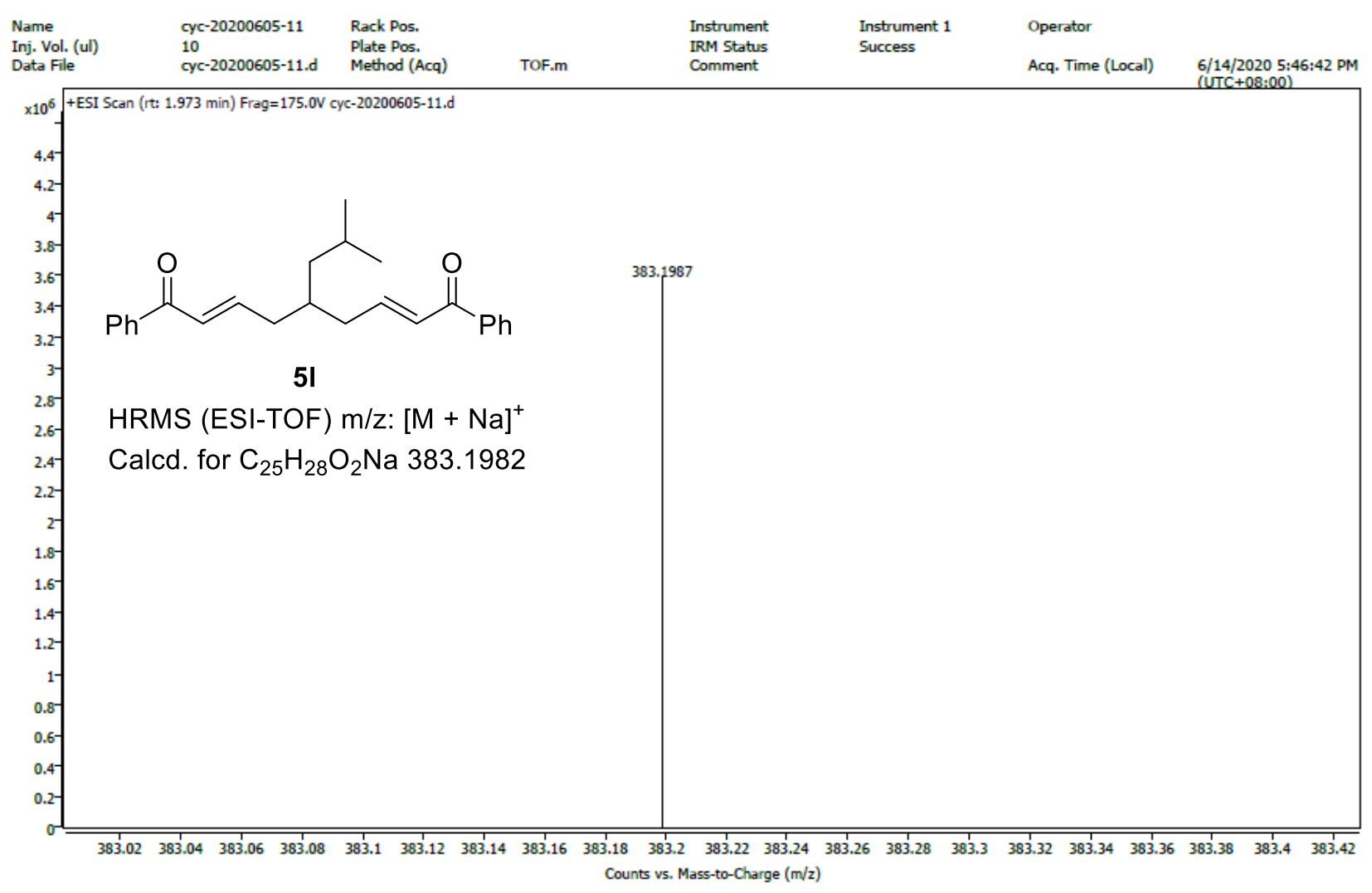


<smiles>O=C(/C=C/CC(C/C=C/C(=O)c1ccc2ccccc2c1)c1ccc(Cl)cc1)c1ccc2ccccc2c1</smiles>

${ }^{1} \mathrm{H}$ NMR $\left(400 \mathrm{MHz}, \mathrm{CDCl}_{3}\right)$

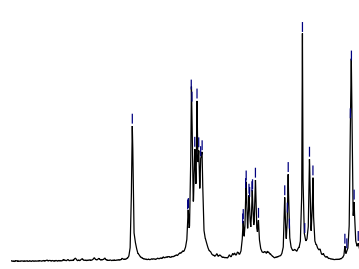

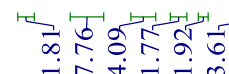
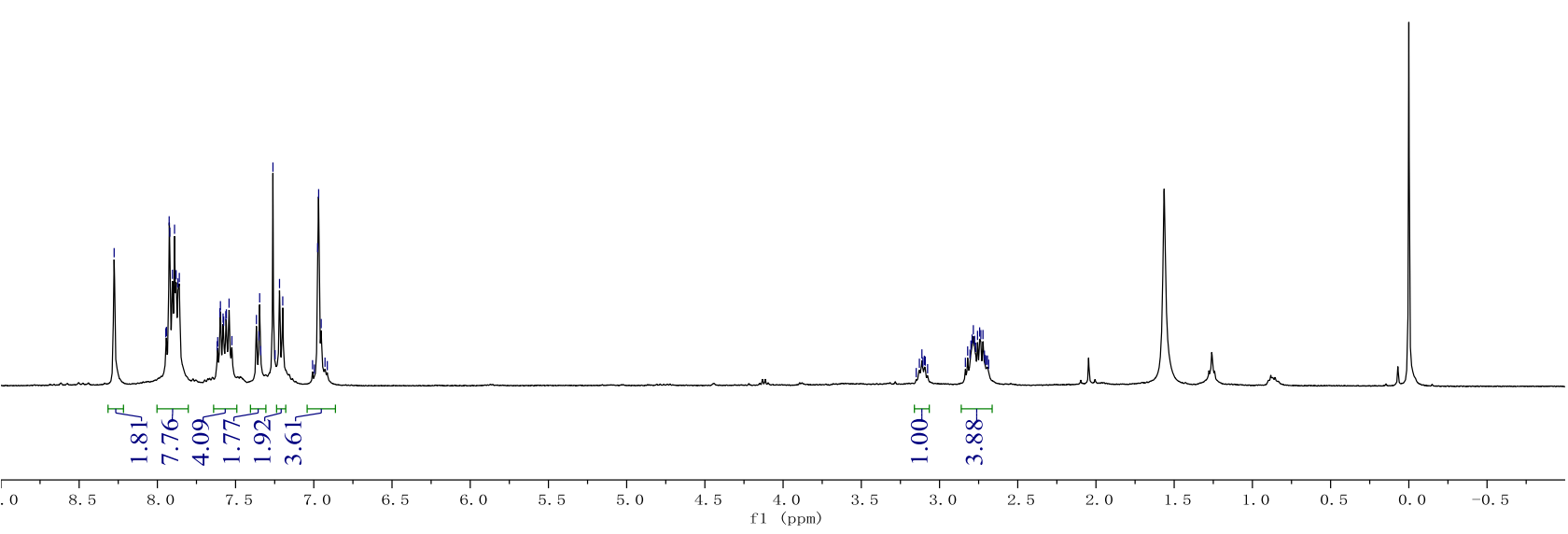

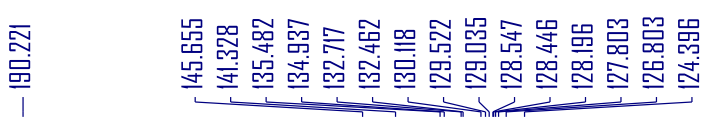

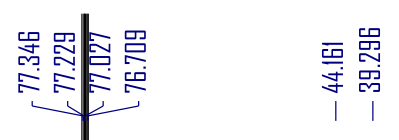

言

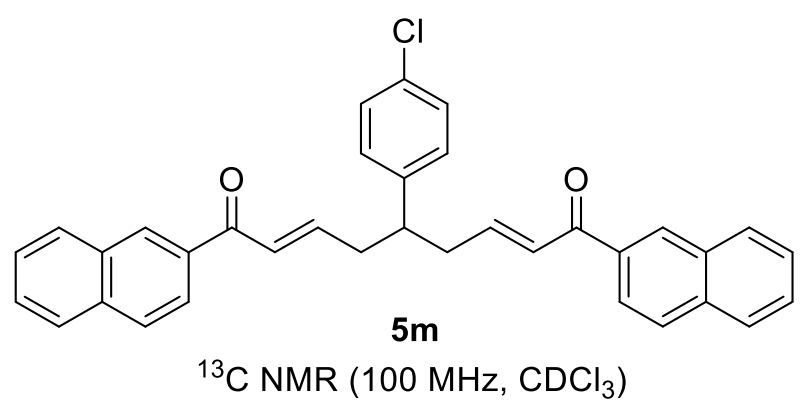

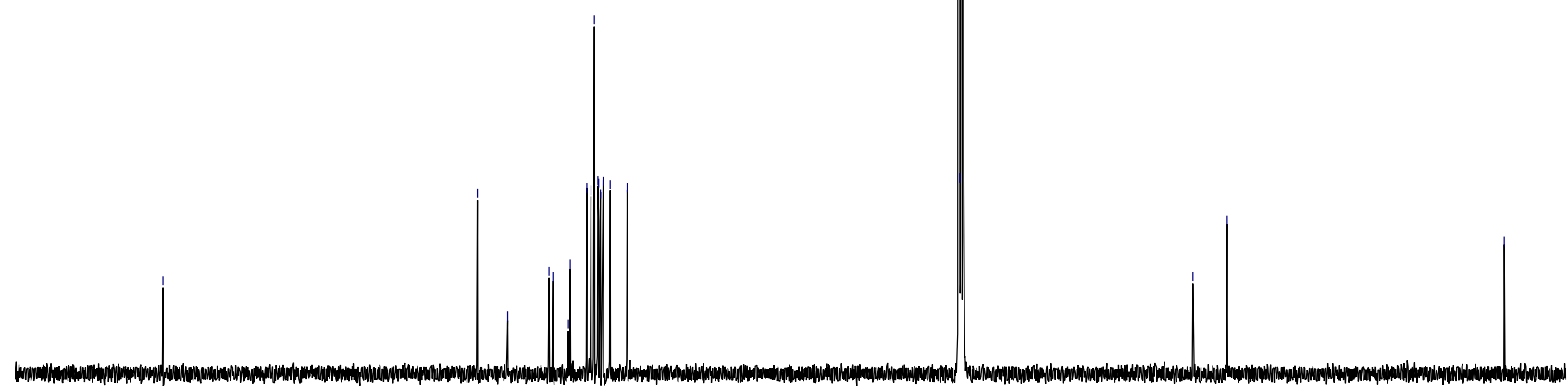

$$
210
$$
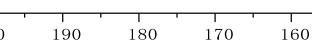

100 
<smiles>O=C(/C=C/CC(C/C=C/C(=O)c1ccc2ccccc2c1)c1ccc(Cl)cc1)c1ccc2ccccc2c1</smiles>

$5 \mathrm{~m}$

HRMS (ESI-TOF) $\mathrm{m} / \mathrm{z}:[\mathrm{M}+\mathrm{Na}]^{+}$

Calcd. for $\mathrm{C}_{35} \mathrm{H}_{27} \mathrm{Cl}^{35} \mathrm{O}_{2} \mathrm{Na} 537.1592$

Calcd. for $\mathrm{C}_{35} \mathrm{H}_{27} \mathrm{Cl}^{37} \mathrm{O}_{2} \mathrm{Na} 539.1562$
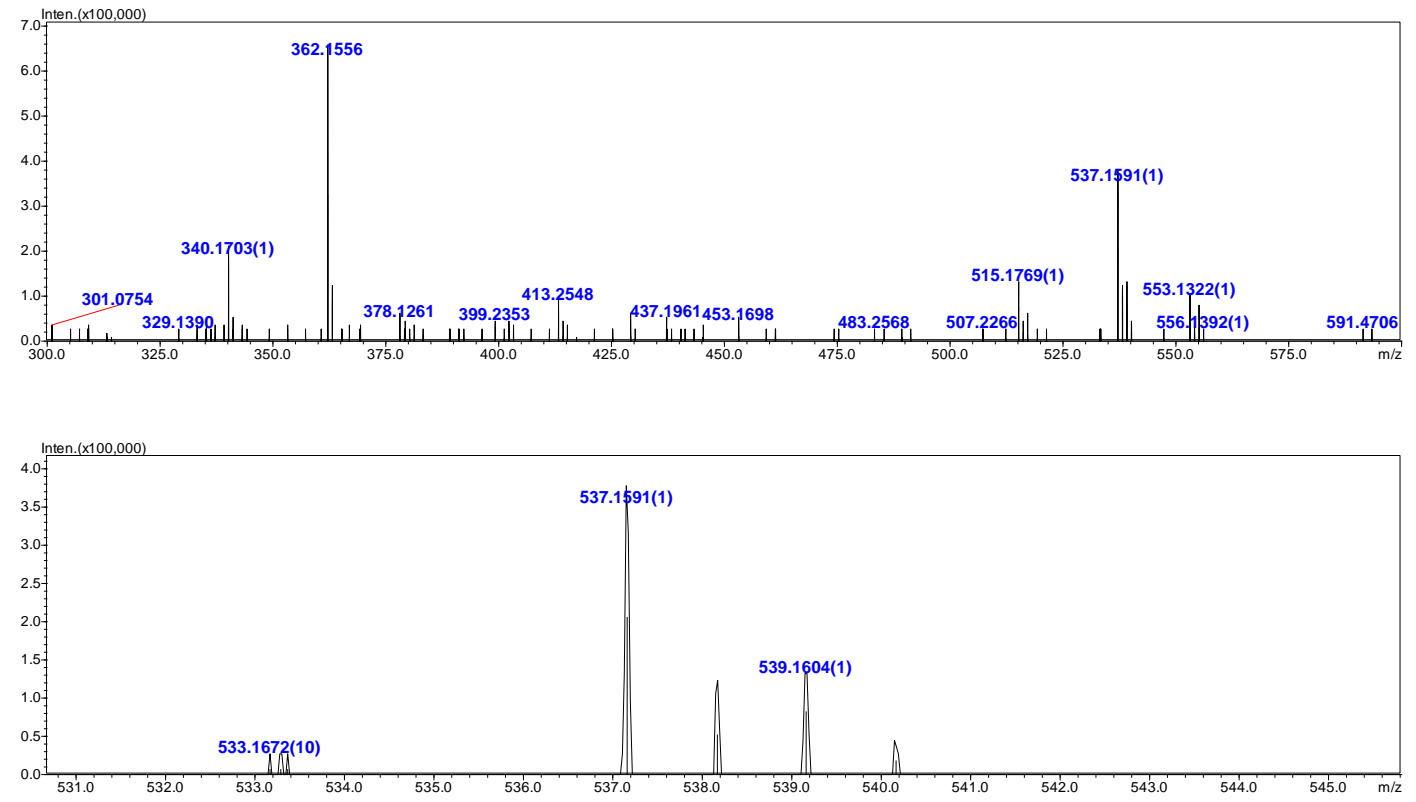


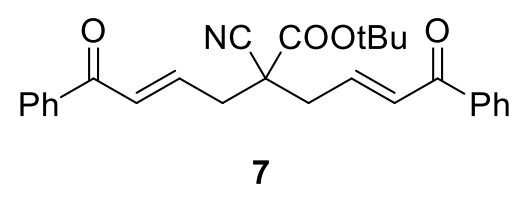

${ }^{1} \mathrm{H}$ NMR $\left(400 \mathrm{MHz}, \mathrm{CDCl}_{3}\right)$

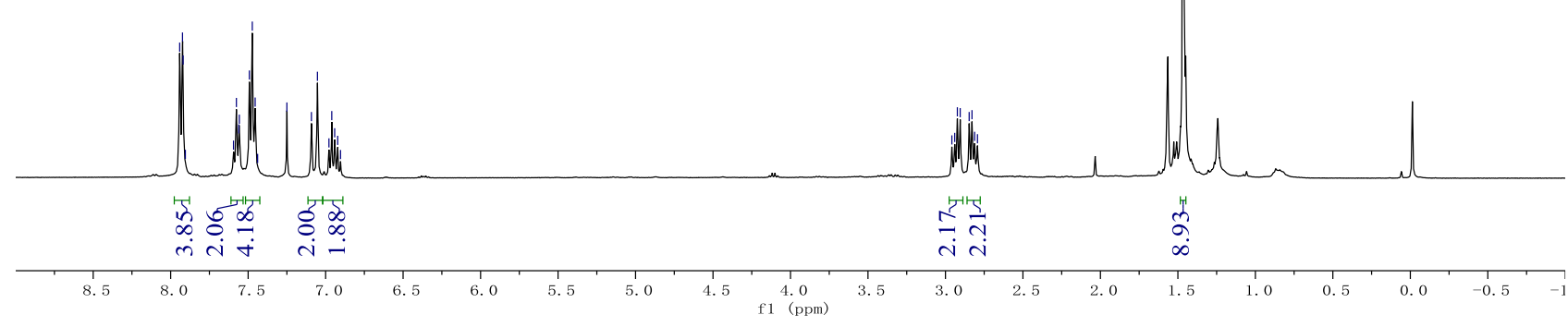

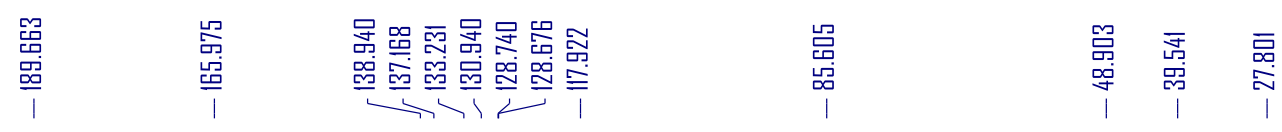

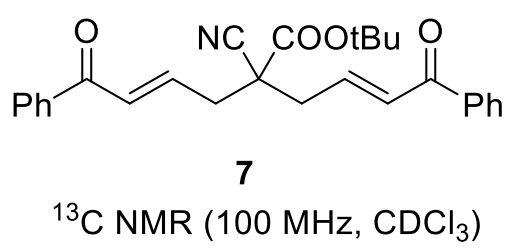

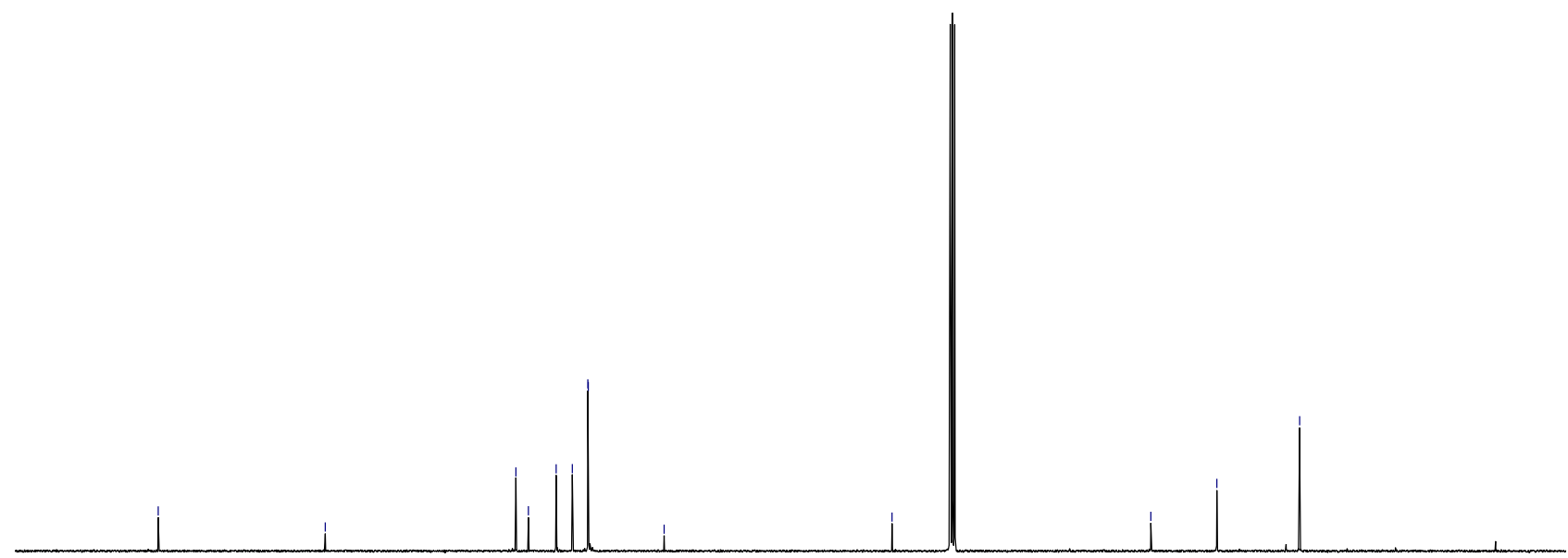

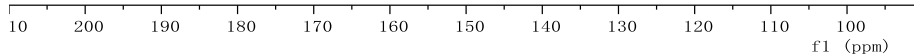




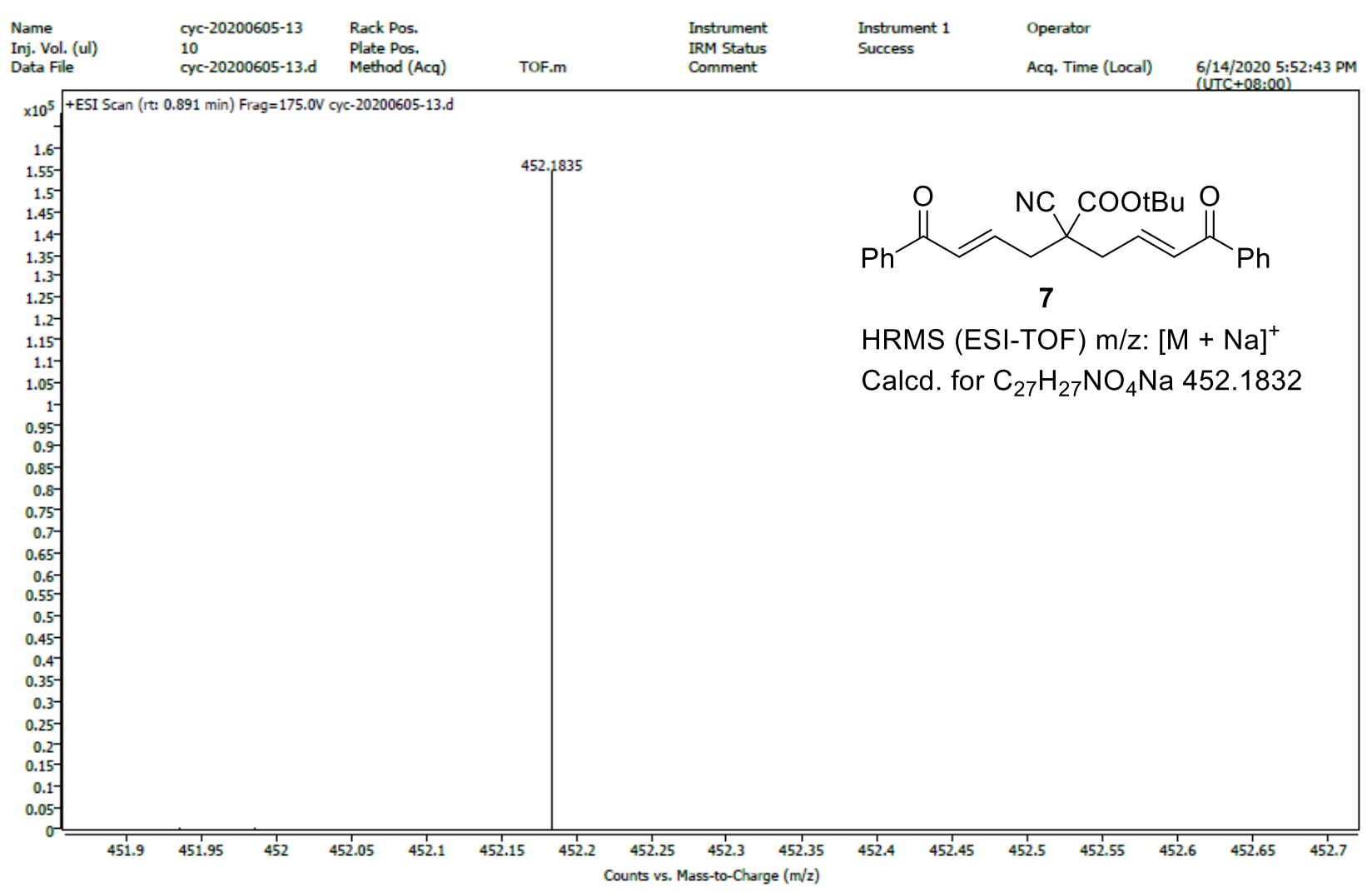


<smiles>O=C(C[C@H]1CCCC=C1C(=O)c1ccccc1)c1ccccc1</smiles>

$2 a$

${ }^{1} \mathrm{H}$ NMR $\left(400 \mid \mathrm{MHz}, \mathrm{CDCl}_{3}\right)$

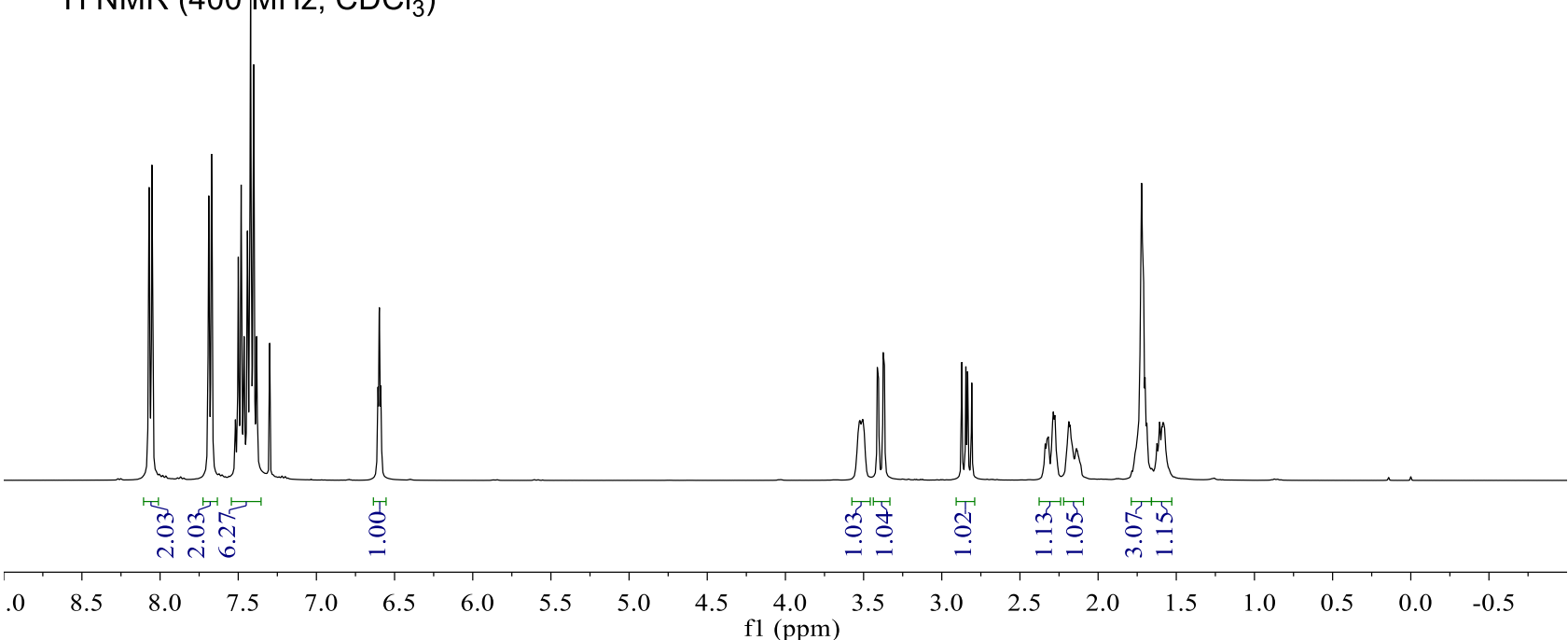

守

亏े

そૅ

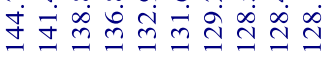

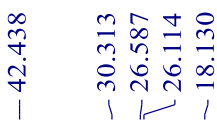<smiles>O=C(C[C@@H]1CCCC=C1C(=O)c1ccccc1)c1ccccc1</smiles>

$2 a$

${ }^{13} \mathrm{C}$ NMR $\left(100 \mathrm{MHz}, \mathrm{CDCl}_{3}\right)$

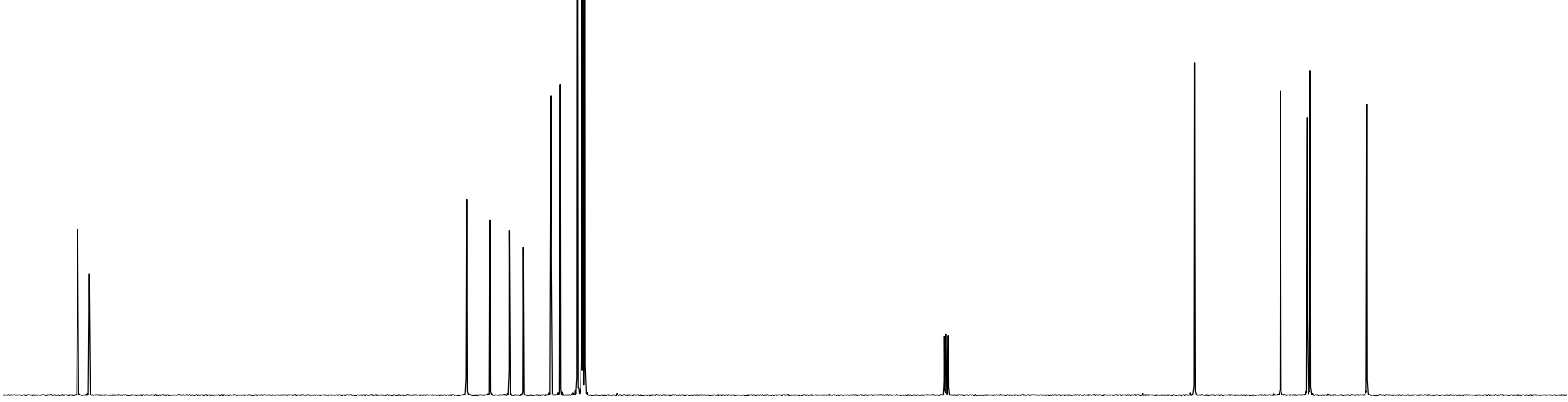

$\begin{array}{lllllllllllllllllllllll}10 & 200 & 190 & 180 & 170 & 160 & 150 & 140 & 130 & 120 & 110 & \begin{array}{c}100 \\ \mathrm{f} 1(\mathrm{ppm})\end{array} & 90 & 80 & 70 & 60 & 50 & 40 & 30 & 20 & 10 & 0 & -\end{array}$ 


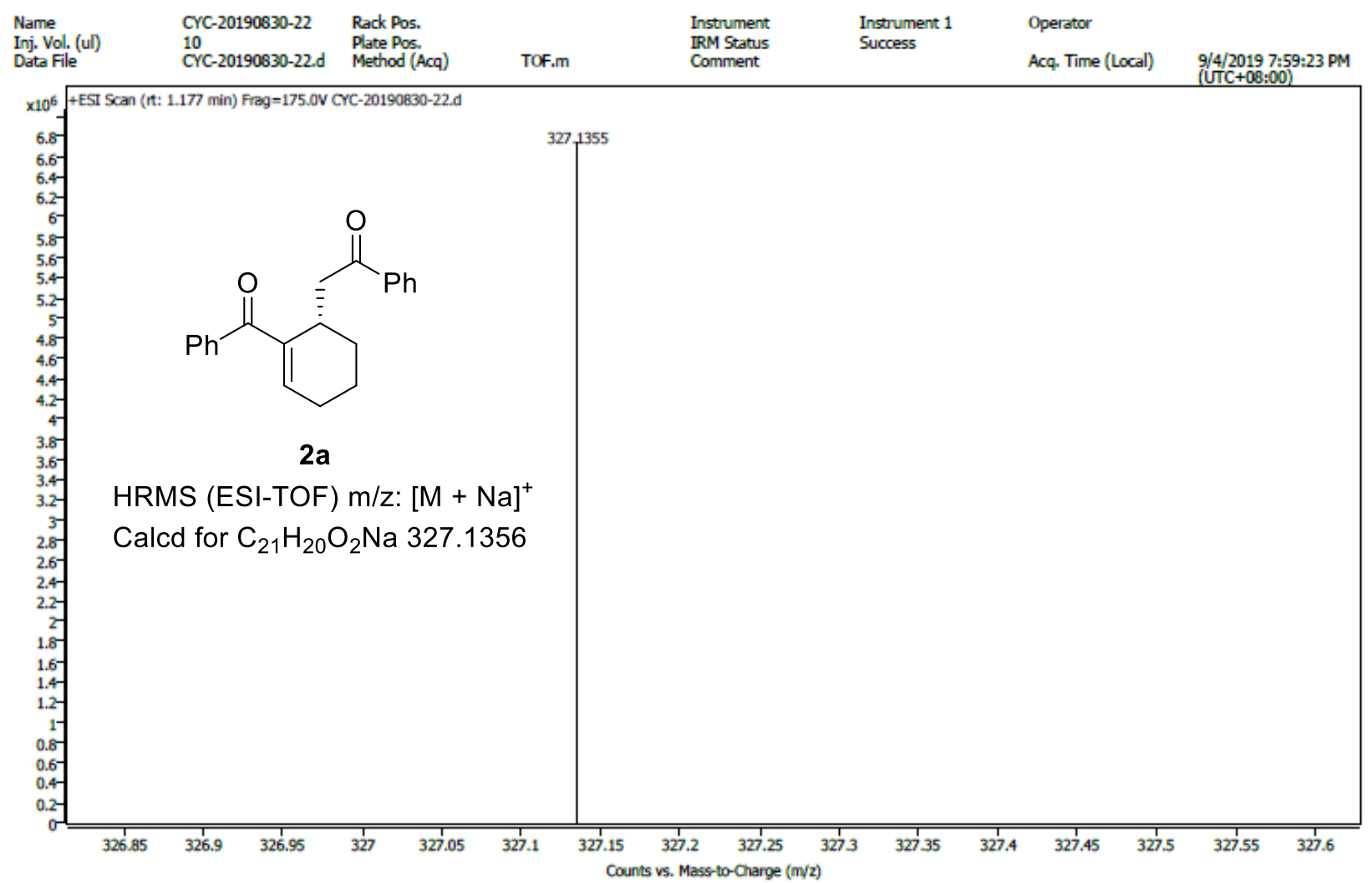




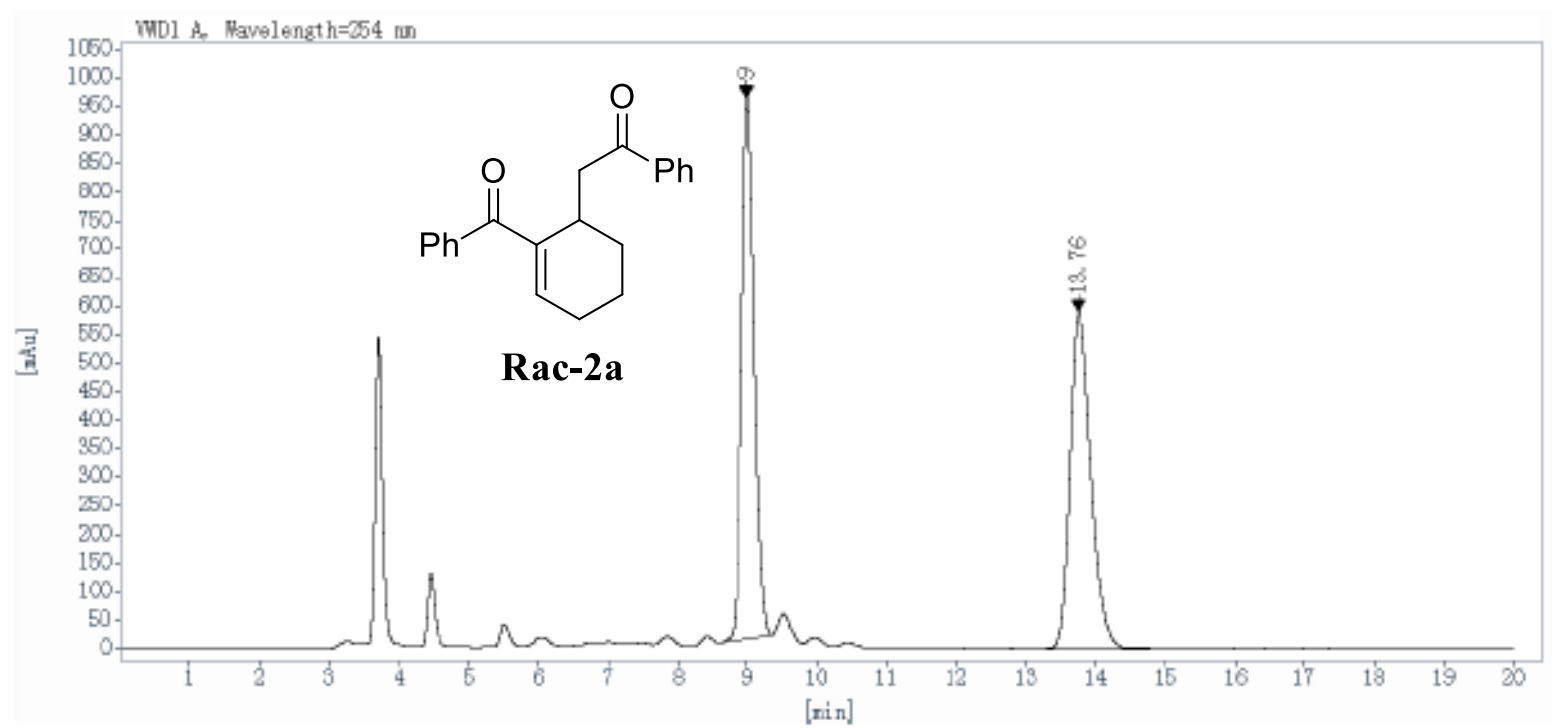

$\begin{array}{cccccc}\begin{array}{c}\text { Ret Time } \\ \text { [min] }\end{array} & \begin{array}{c}\text { Peak } \\ \text { Type }\end{array} & \begin{array}{c}\text { Width } \\ {[\mathrm{min}]}\end{array} & \begin{array}{c}\text { Height } \\ {[\mathrm{mAU}]}\end{array} & \begin{array}{c}\text { Area } \\ {\left[\mathrm{mAU}^{*} \text { s] }\right.}\end{array} & \begin{array}{c}\text { Area } \\ {[\%]}\end{array} \\ 9.000 & \text { BB } & 0.19 & 949.4163 & 11891.0498 & 49.0475 \\ 13.760 & \text { VB R } & 0.32 & 589.7098 & 12352.8770 & 50.9525 \\ & & & \text { Totals: } & 24243.9268 & 100.0000\end{array}$

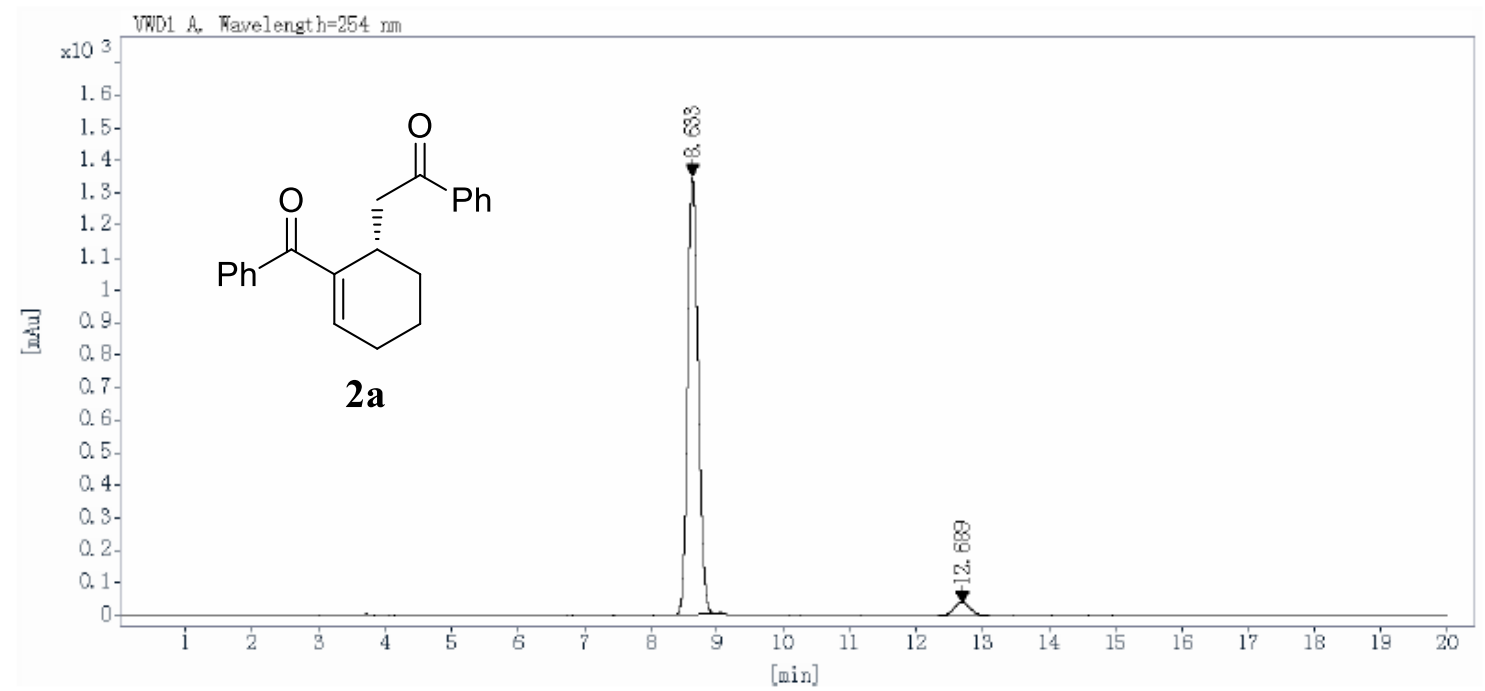

\begin{tabular}{cccccc}
$\begin{array}{c}\text { Ret Time } \\
\text { [min] }\end{array}$ & $\begin{array}{c}\text { Peak } \\
\text { Type }\end{array}$ & $\begin{array}{c}\text { Width } \\
{[\mathrm{min}]}\end{array}$ & $\begin{array}{c}\text { Height } \\
{[\mathrm{mAU}]}\end{array}$ & $\begin{array}{c}\text { Area } \\
{[\mathrm{mAU} \text { *s] }}\end{array}$ & $\begin{array}{c}\text { Area } \\
{[\%]}\end{array}$ \\
\hline 8.633 & BV R & 0.17 & 1347.3600 & 14914.5029 & 95.7034 \\
12.689 & BB & 0.26 & 39.5748 & 669.5833 & 4.2966 \\
& & & Totals: & 15584.0862 & 100.0000
\end{tabular}


<smiles>Cc1ccc(C(=O)C[C@H]2CCCC=C2C(=O)c2ccc(C)cc2)cc1</smiles>

2b

${ }^{1} \mathrm{H}$ NMR $\left(400 \mathrm{MHz}, \mathrm{CDCl}_{3}\right)$

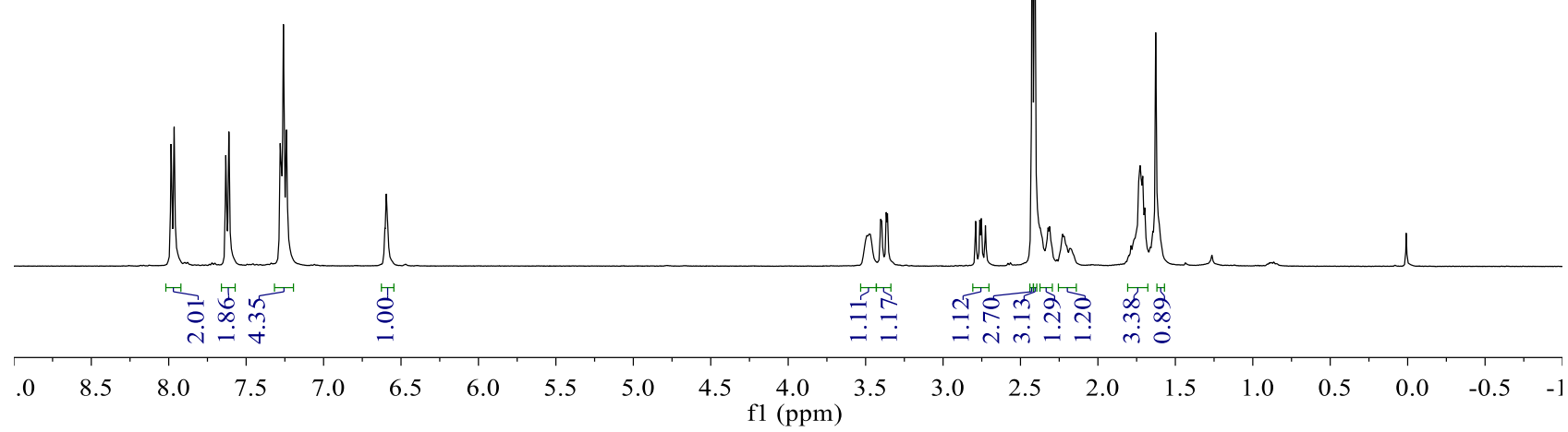

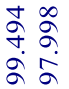

के ڤे

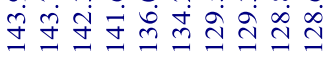<smiles>Cc1ccc(C(=O)C[C@H]2CCCC=C2C(=O)c2ccc(C)cc2)cc1</smiles>

2b

${ }^{13} \mathrm{C} \mathrm{NMR}\left(100 \mathrm{MHz}, \mathrm{CDCl}_{3}\right)$

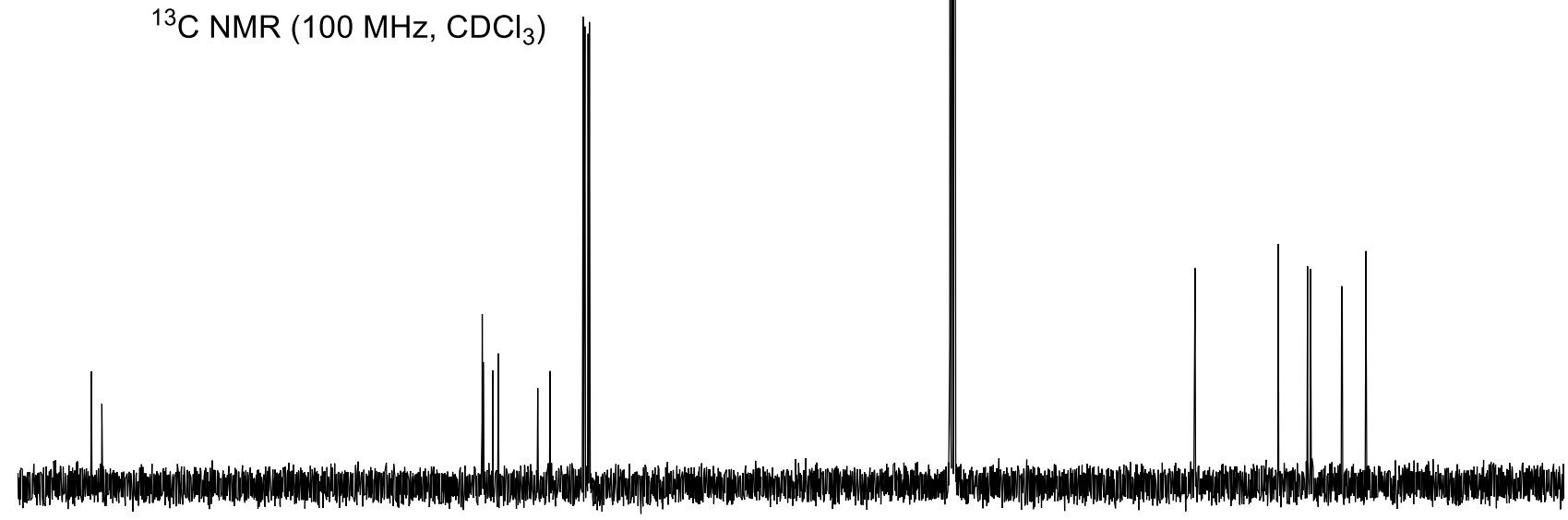

$\begin{array}{llllllllllllllllllllllll}10 & 200 & 190 & 180 & 170 & 160 & 150 & 140 & 130 & 120 & 110 & 100 & 90 & 80 & 70 & 60 & 50 & 40 & 30 & 20 & 10 & 0 & -\end{array}$ 


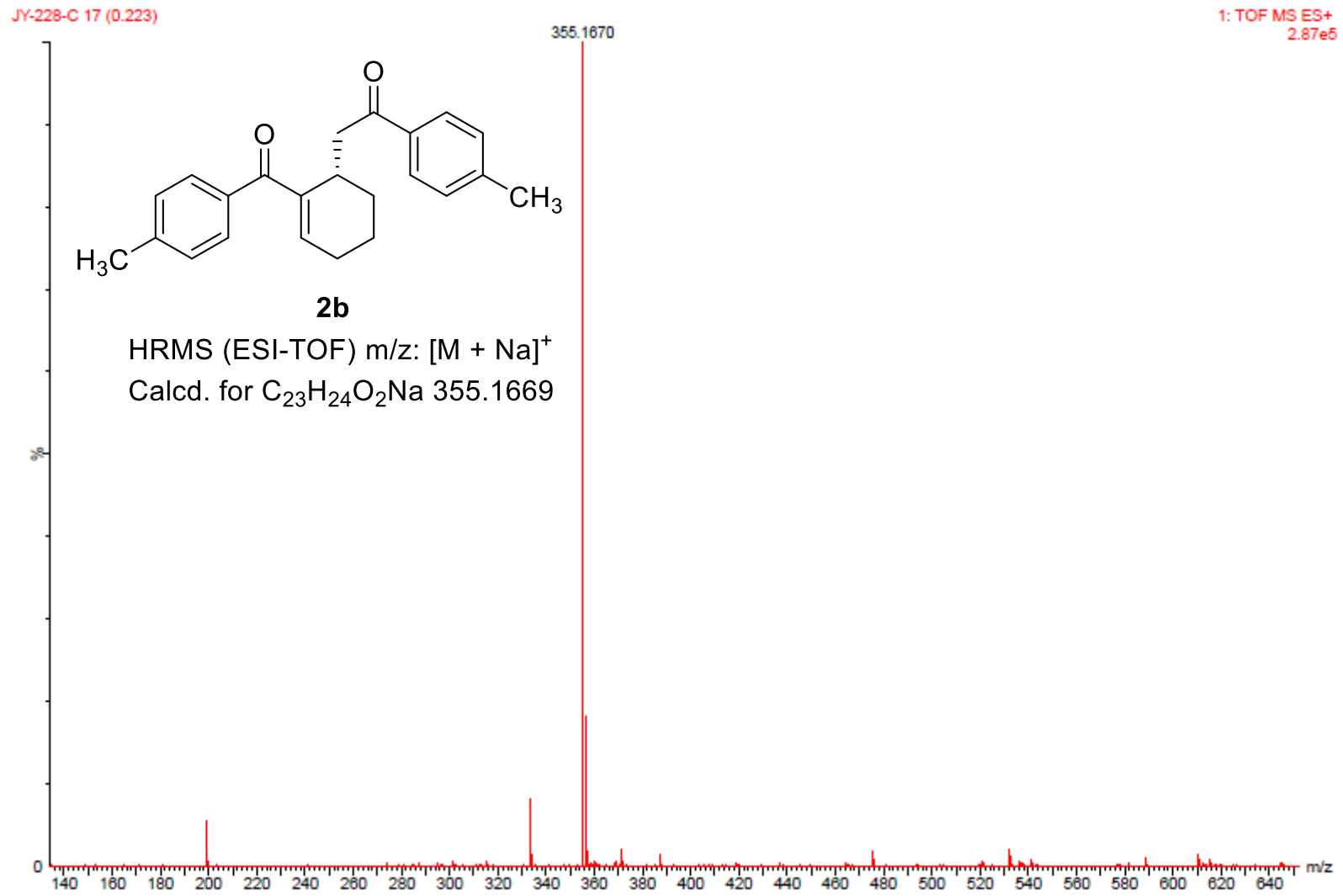




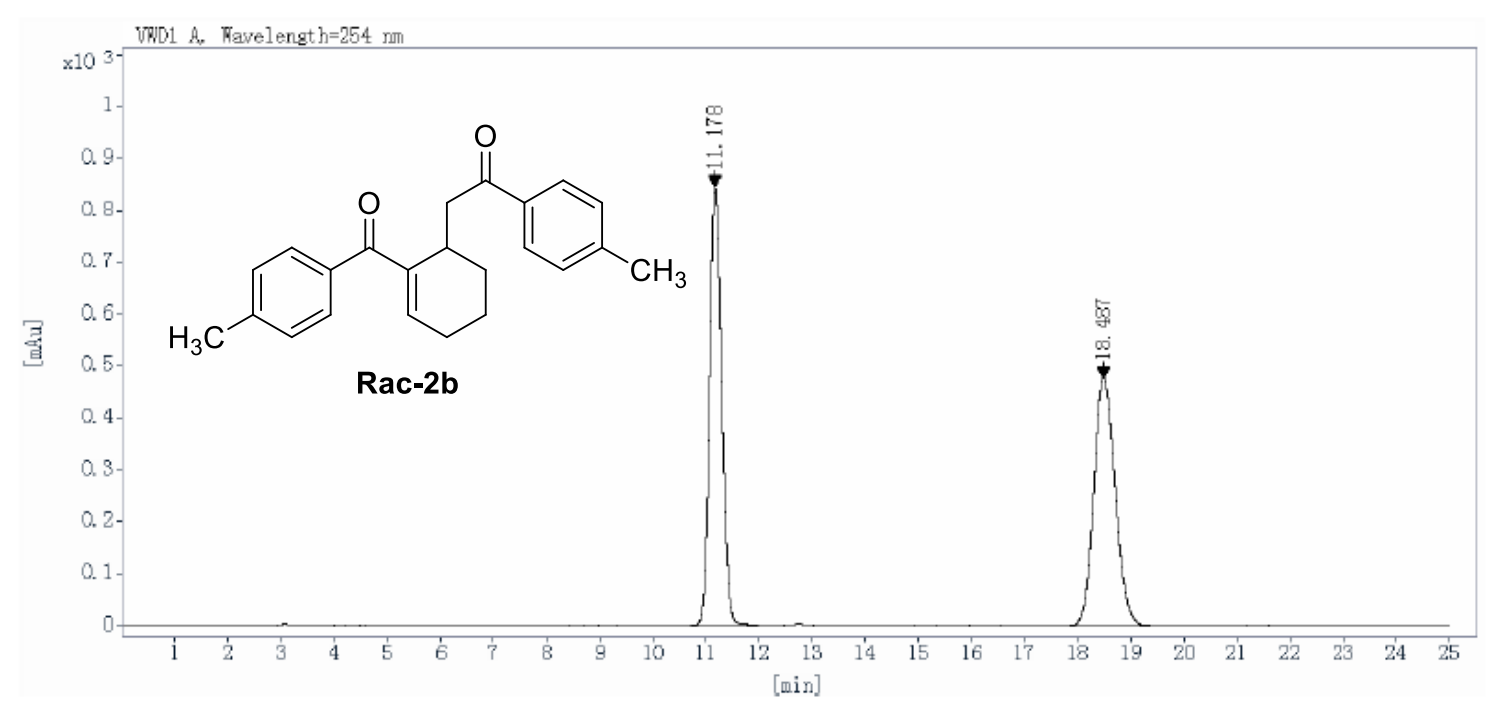

$\begin{array}{cccccc}\begin{array}{c}\text { Ret Time } \\ \text { [min] }\end{array} & \begin{array}{c}\text { Peak } \\ \text { Type }\end{array} & \begin{array}{c}\text { Width } \\ {[\mathrm{min}]}\end{array} & \begin{array}{c}\text { Height } \\ {[\mathrm{mAU}]}\end{array} & \begin{array}{c}\text { Area } \\ {\left[\mathrm{mAU} \mathbf{U}^{*} \text { s] }\right.}\end{array} & \begin{array}{c}\text { Area } \\ {[\%]}\end{array} \\ 11.178 & \text { BB } & 0.25 & 844.1830 & 13367.1826 & 50.1208 \\ 18.487 & \text { BB } & 0.43 & 476.8644 & 13302.7305 & 49.8792 \\ & & & \text { Totals: } & 26669.9131 & 100.0000\end{array}$

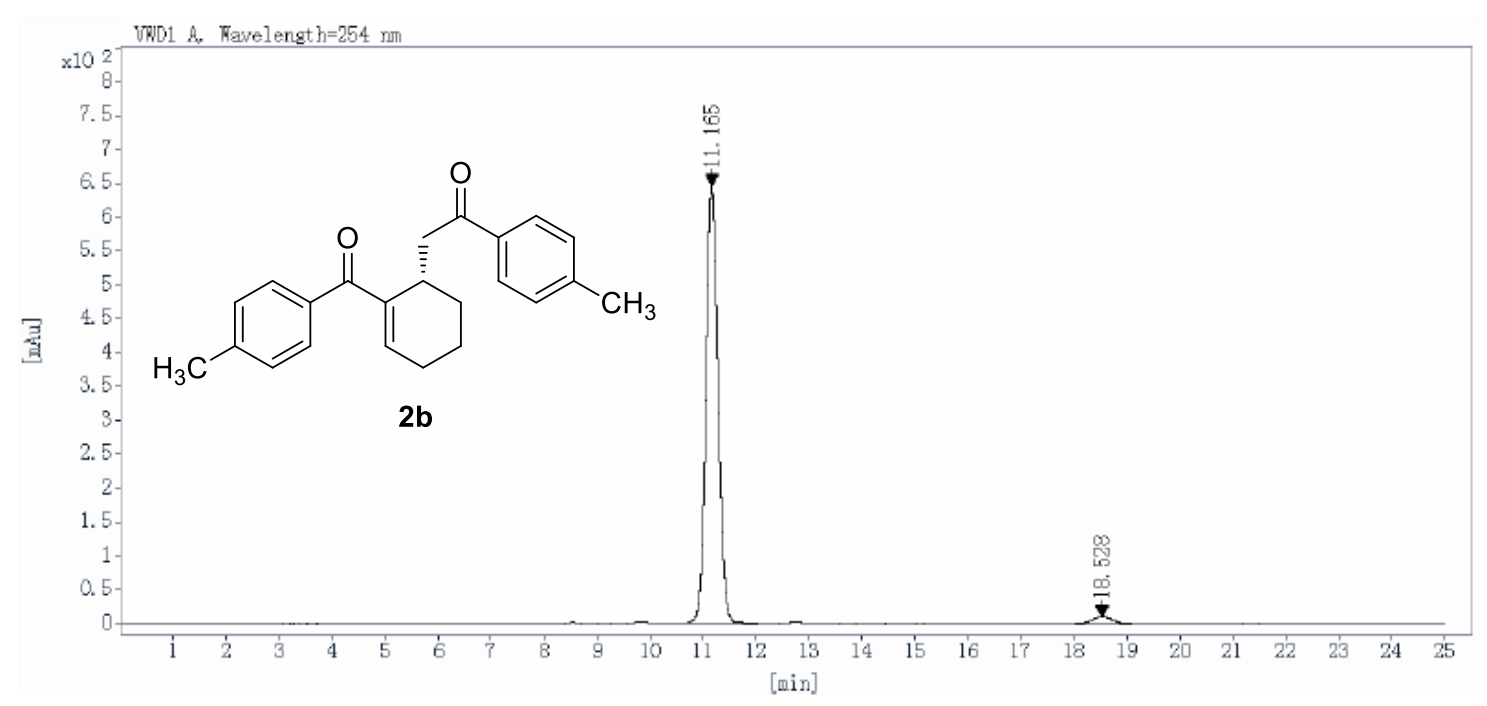

$\begin{array}{cccccc}\begin{array}{c}\text { Ret Time } \\ \text { [min] }\end{array} & \begin{array}{c}\text { Peak } \\ \text { Type }\end{array} & \begin{array}{c}\text { Width } \\ {[\mathrm{min}]}\end{array} & \begin{array}{c}\text { Height } \\ {[\mathrm{mAU}]}\end{array} & \begin{array}{c}\text { Area } \\ {\left[\mathrm{mAU} \mathbf{A U}^{*} \text { ] }\right.}\end{array} & \begin{array}{c}\text { Area } \\ {[\%]}\end{array} \\ 11.165 & \text { BBA } & 0.25 & 645.9575 & 10286.6123 & 97.3380 \\ 18.528 & \text { BB } & 0.43 & 10.1540 & 281.3170 & 2.6620 \\ & & & \text { Totals: } & 10567.9294 & 100.0000\end{array}$




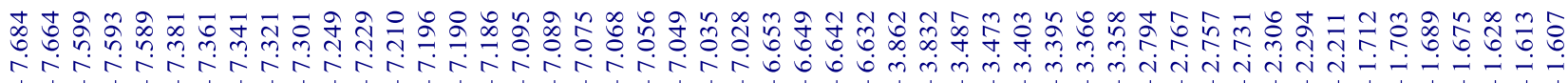<smiles>COc1cccc(C(=O)C[C@@H]2CCCC=C2C(=O)c2cccc(OC)c2)c1</smiles>

$$
\stackrel{\text { ac }}{{ }^{1} \mathrm{H} \text { NMR }\left(400 \mathrm{MHz}, \mathrm{CDCl}_{3}\right)}
$$
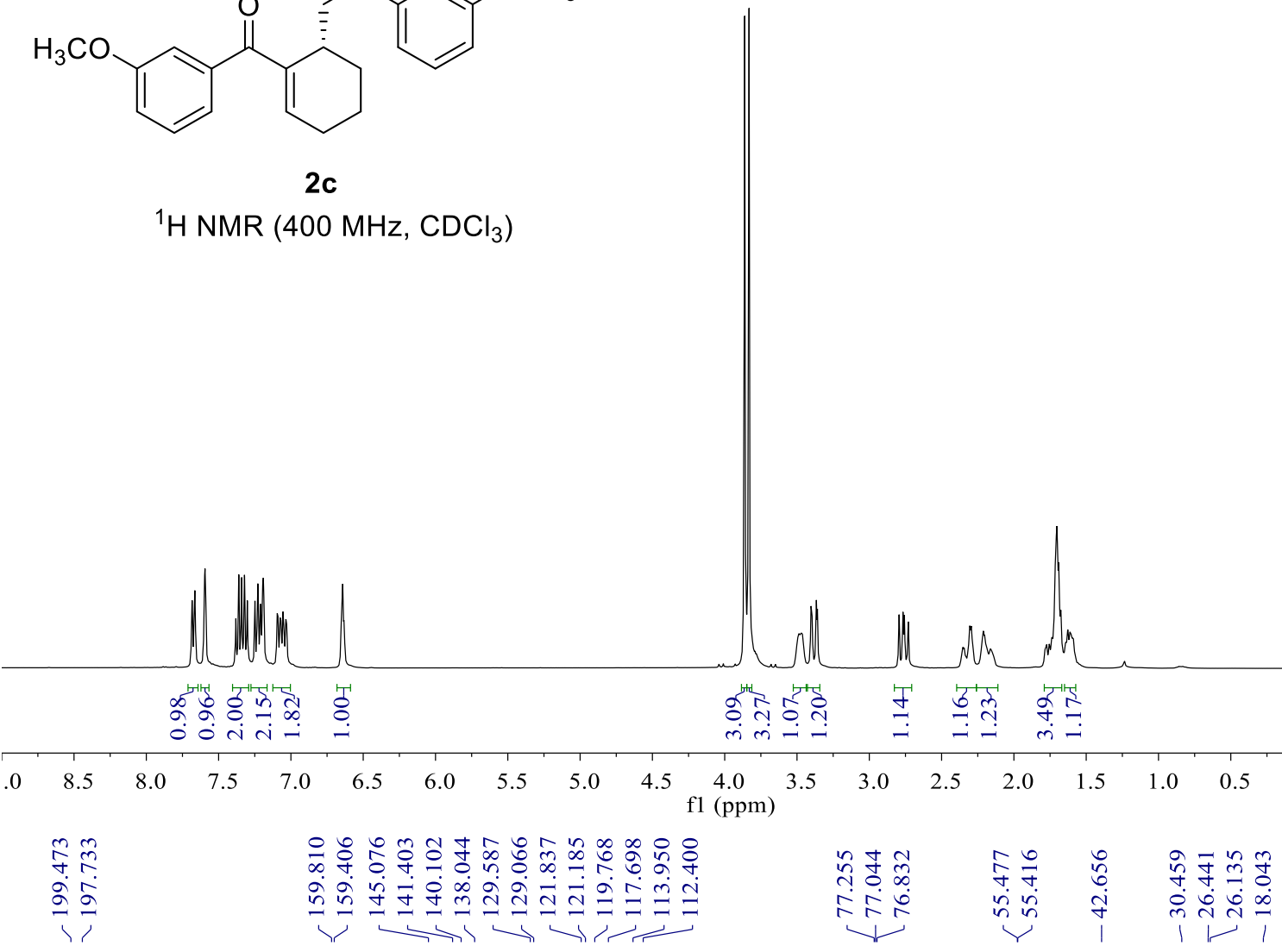<smiles>COc1cccc(C(=O)C[C@@H]2CCCC=C2C(=O)c2cccc(OC)c2)c1</smiles>

ic

${ }^{13} \mathrm{C}$ NMR (150 MHz, $\mathrm{CDCl}_{3}$ )
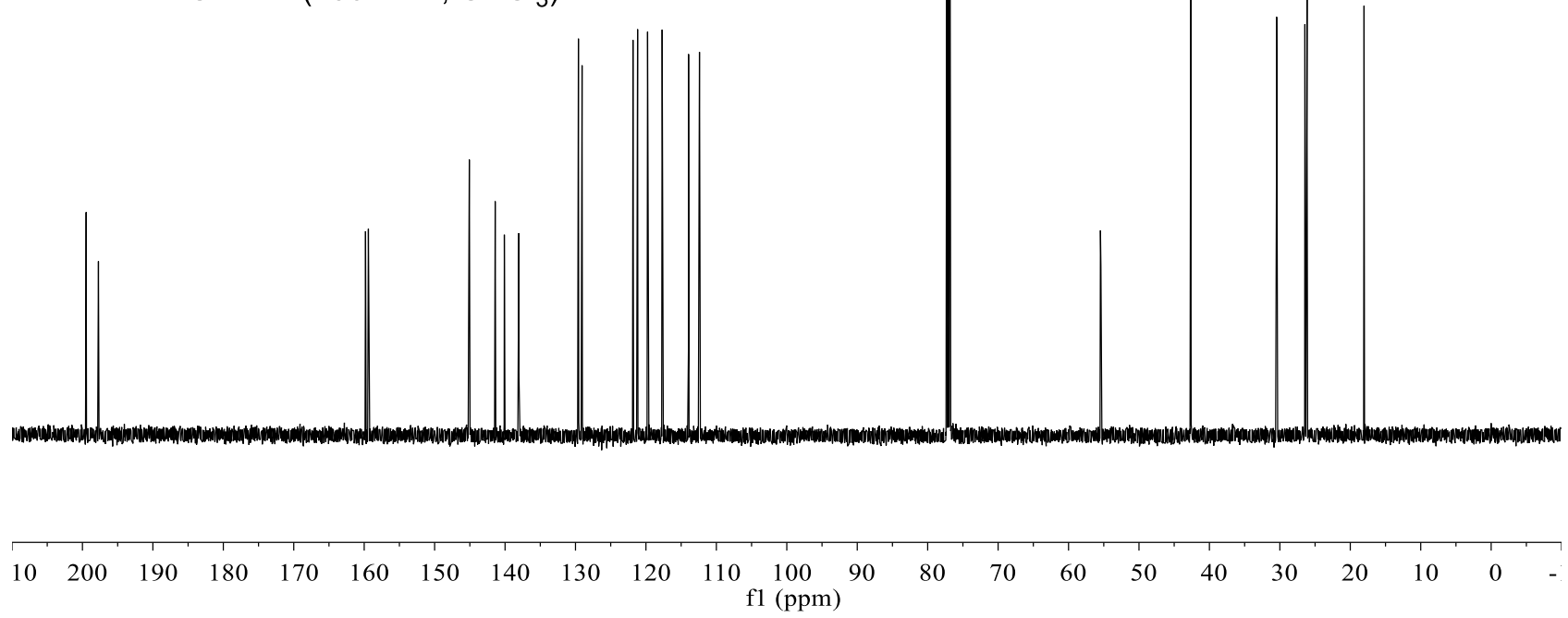

S56 


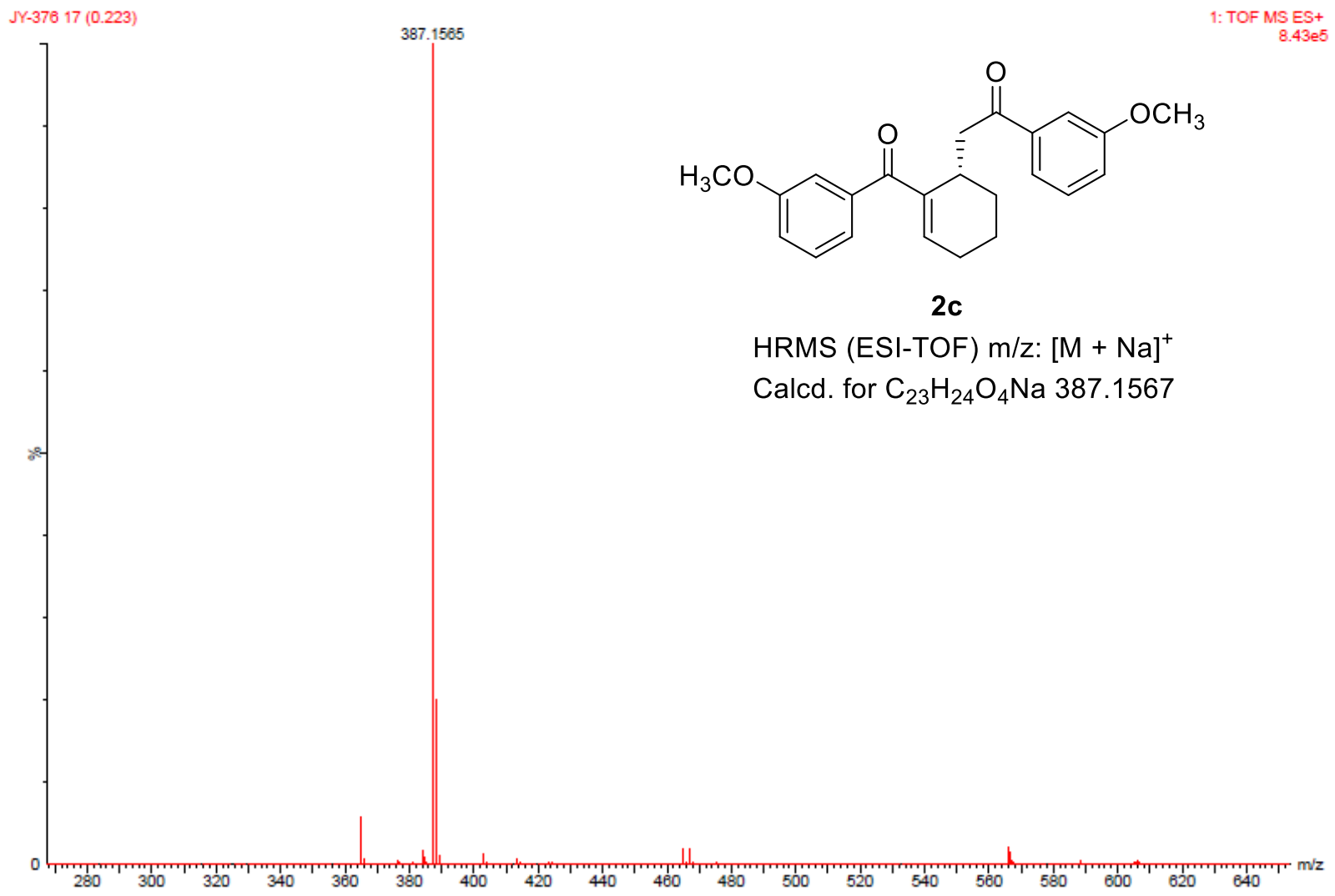




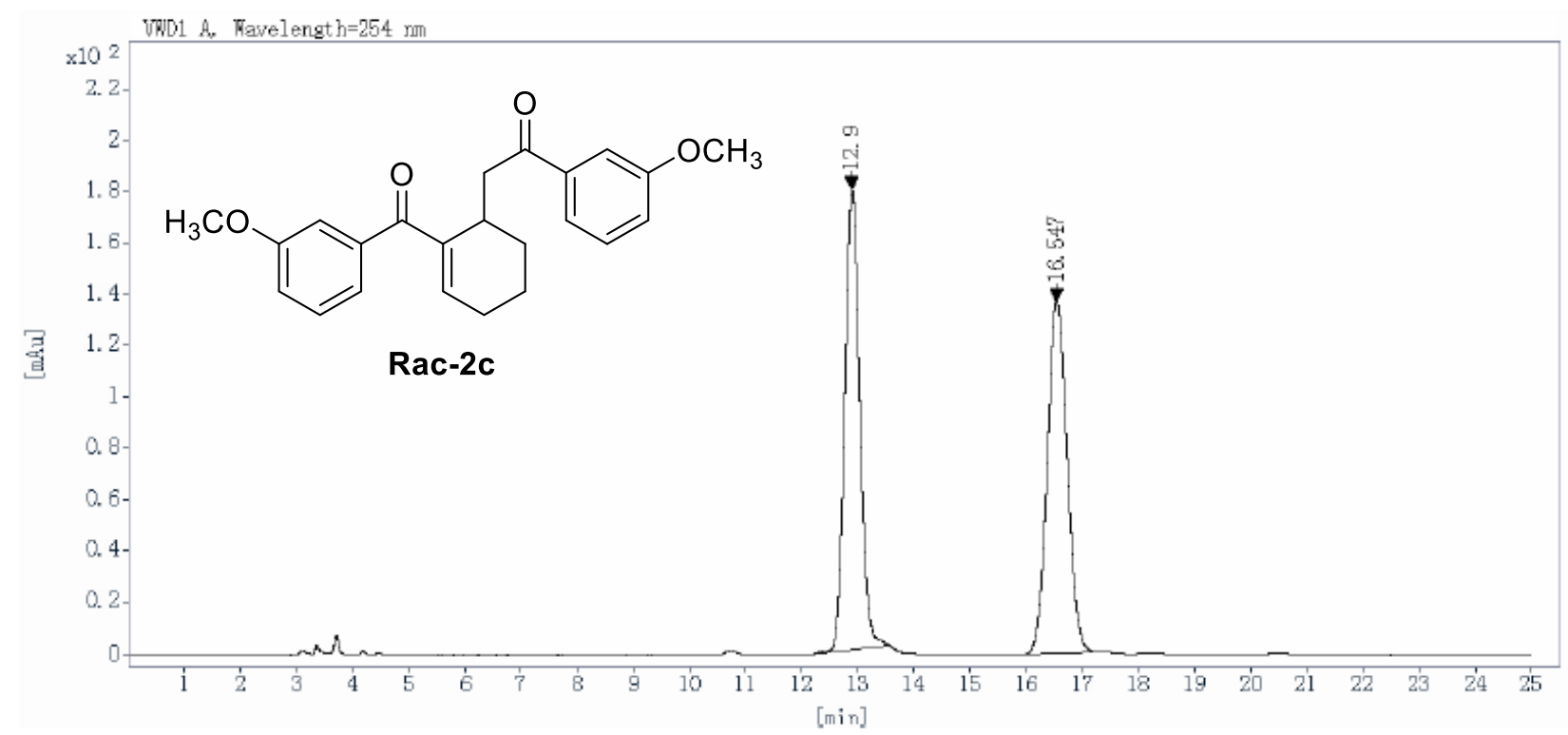

$\begin{array}{cccccc}\begin{array}{c}\text { Ret Time } \\ \text { [min] }\end{array} & \begin{array}{c}\text { Peak } \\ \text { Type }\end{array} & \begin{array}{c}\text { Width } \\ {[\mathrm{min}]}\end{array} & \begin{array}{c}\text { Height } \\ {[\mathrm{mAU}]}\end{array} & \begin{array}{c}\text { Area } \\ {\left[\mathrm{mAU}^{*} \mathbf{s}\right]}\end{array} & \begin{array}{c}\text { Area } \\ {[\%]}\end{array} \\ 12.900 & \text { BBA } & 0.29 & 178.8918 & 3330.3296 & 49.8149 \\ 16.547 & \text { BB } & 0.38 & \begin{array}{c}137.0138 \\ \text { Totals: }\end{array} & 3355.0806 & 50.1851 \\ & & & & & \end{array}$

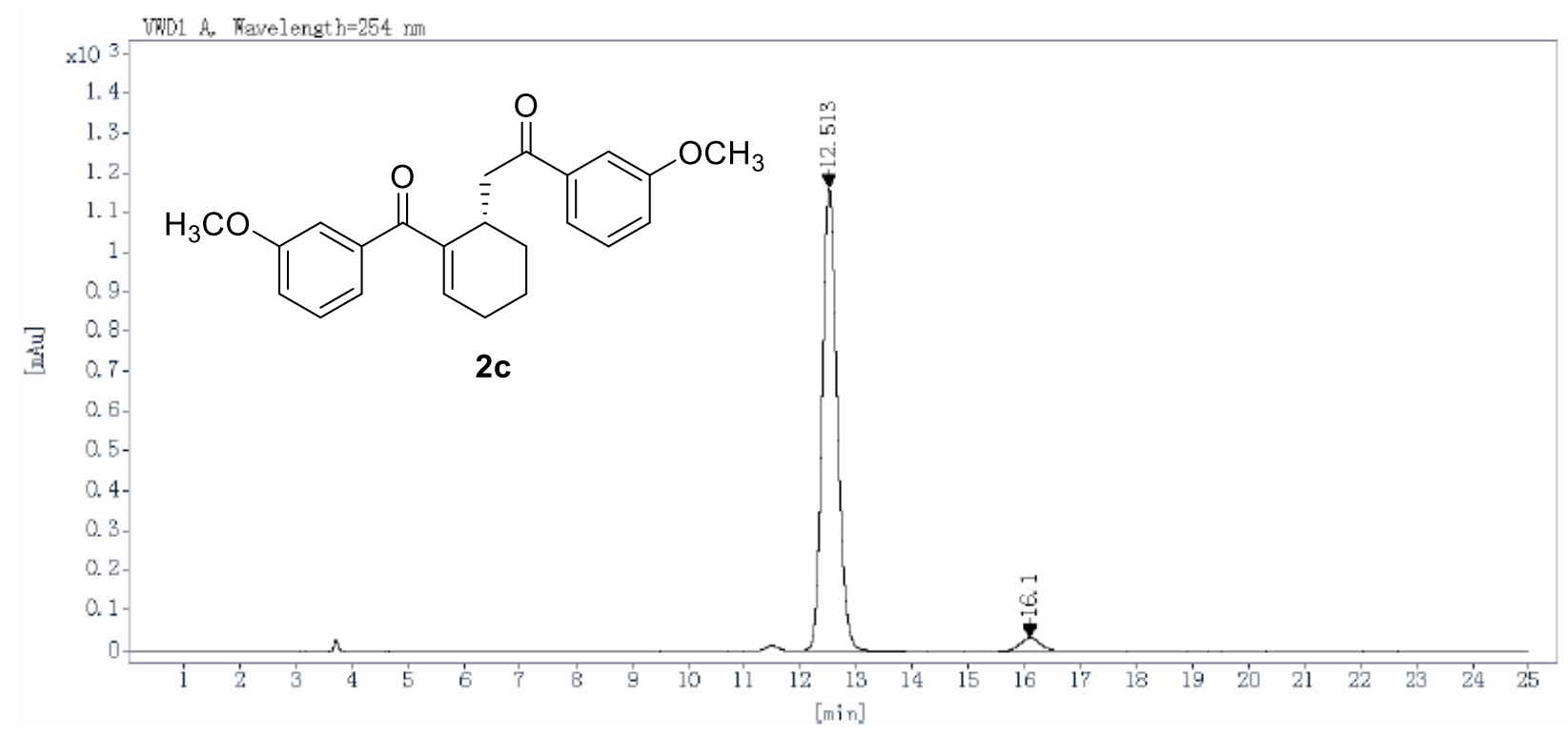

\begin{tabular}{|c|c|c|c|c|c|}
\hline $\begin{array}{l}\text { Ret Time } \\
\text { [min] }\end{array}$ & $\begin{array}{l}\text { Peak } \\
\text { Type }\end{array}$ & $\begin{array}{l}\text { Width } \\
\text { [min] }\end{array}$ & $\begin{array}{l}\text { Height } \\
\text { [mAU] }\end{array}$ & $\begin{array}{c}\text { Area } \\
{\left[\mathbf{m A U}^{*} \mathrm{~s}\right]}\end{array}$ & $\begin{array}{c}\text { Area } \\
{[\%]}\end{array}$ \\
\hline 12.513 & BB & 0.29 & 1162.2616 & 21621.1230 & 96.0747 \\
\hline 16.100 & BB & 0.40 & 33.7654 & 883.3745 & 3.9253 \\
\hline & & & Totals: & 22504.4976 & 100.0000 \\
\hline
\end{tabular}


<smiles>O=C(C[C@@H]1CCCC=C1C(=O)c1ccc(Br)cc1)c1ccc(Br)cc1</smiles>

2d

${ }^{1} \mathrm{H} \mathrm{NMR}\left(400 \mathrm{MHz}, \mathrm{CDCl}_{3}\right)$

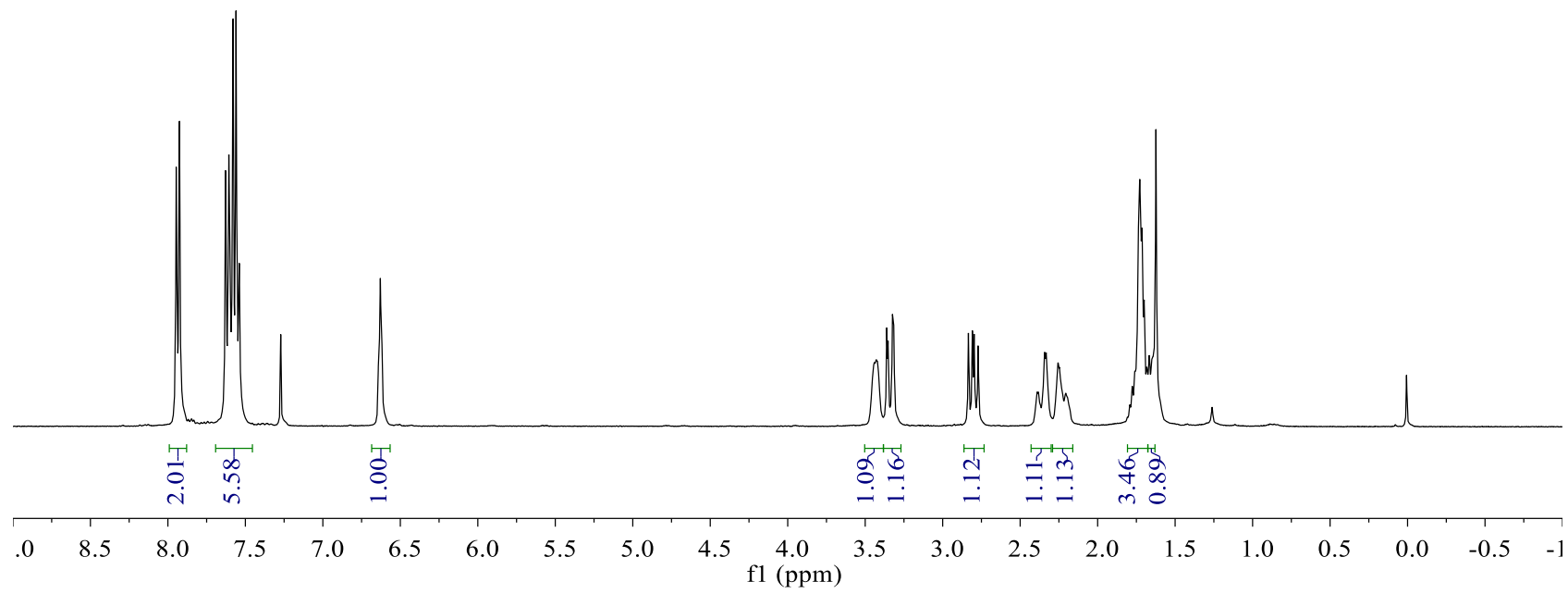

赵

$\stackrel{\infty}{\circ} \stackrel{0}{2}$

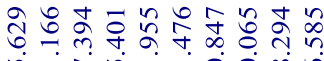

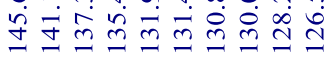<smiles>O=C(C[C@@H]1CCCC=C1C(=O)c1ccc(Br)cc1)c1ccc(Br)cc1</smiles>

2d

${ }^{13} \mathrm{C} \mathrm{NMR}\left(100 \mathrm{MHz}, \mathrm{CDCl}_{3}\right)$

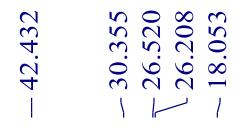

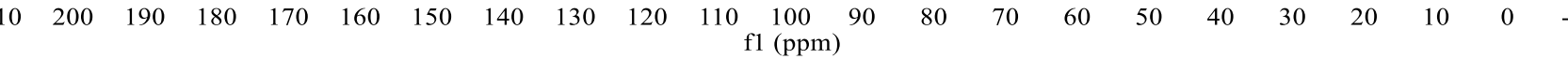




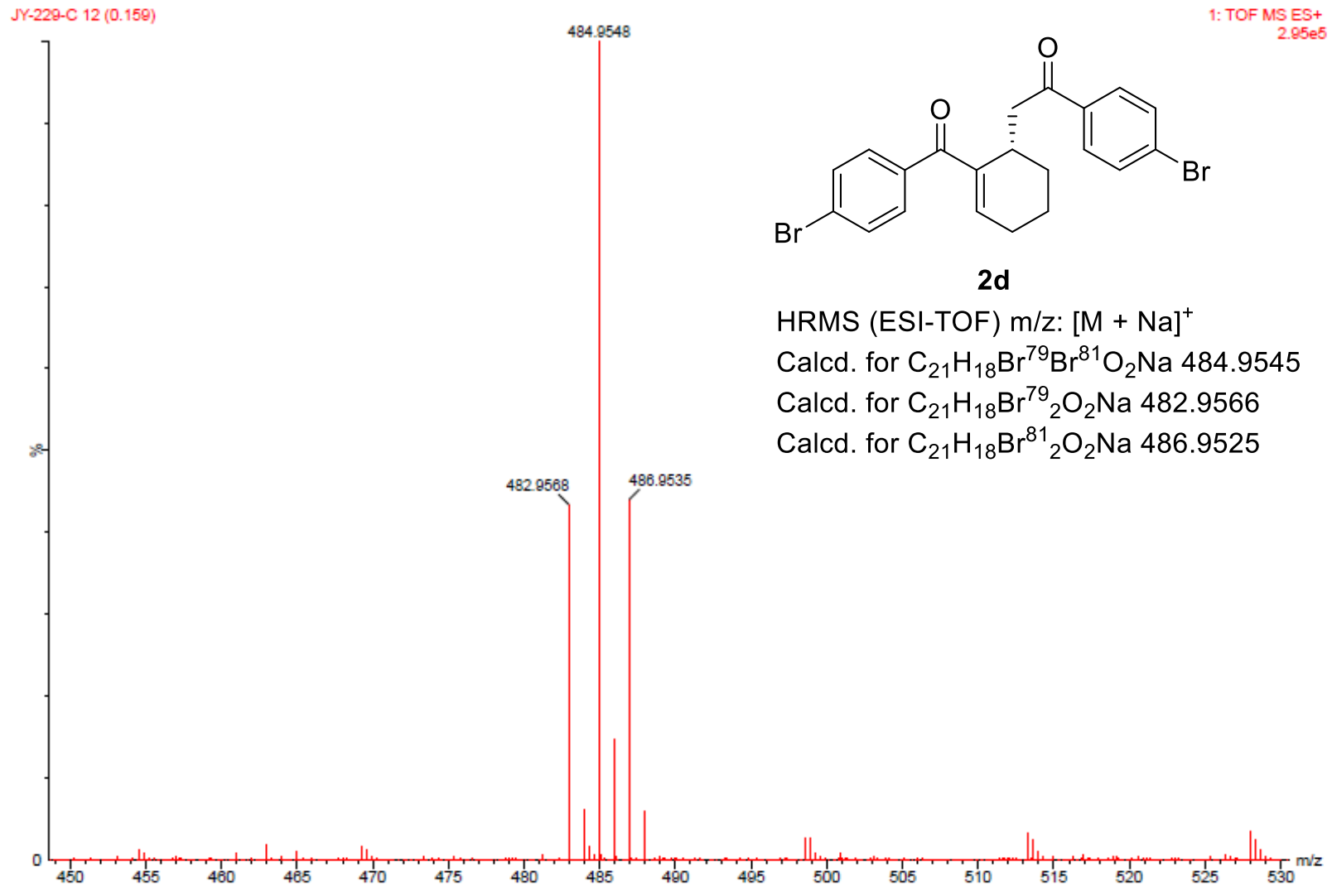




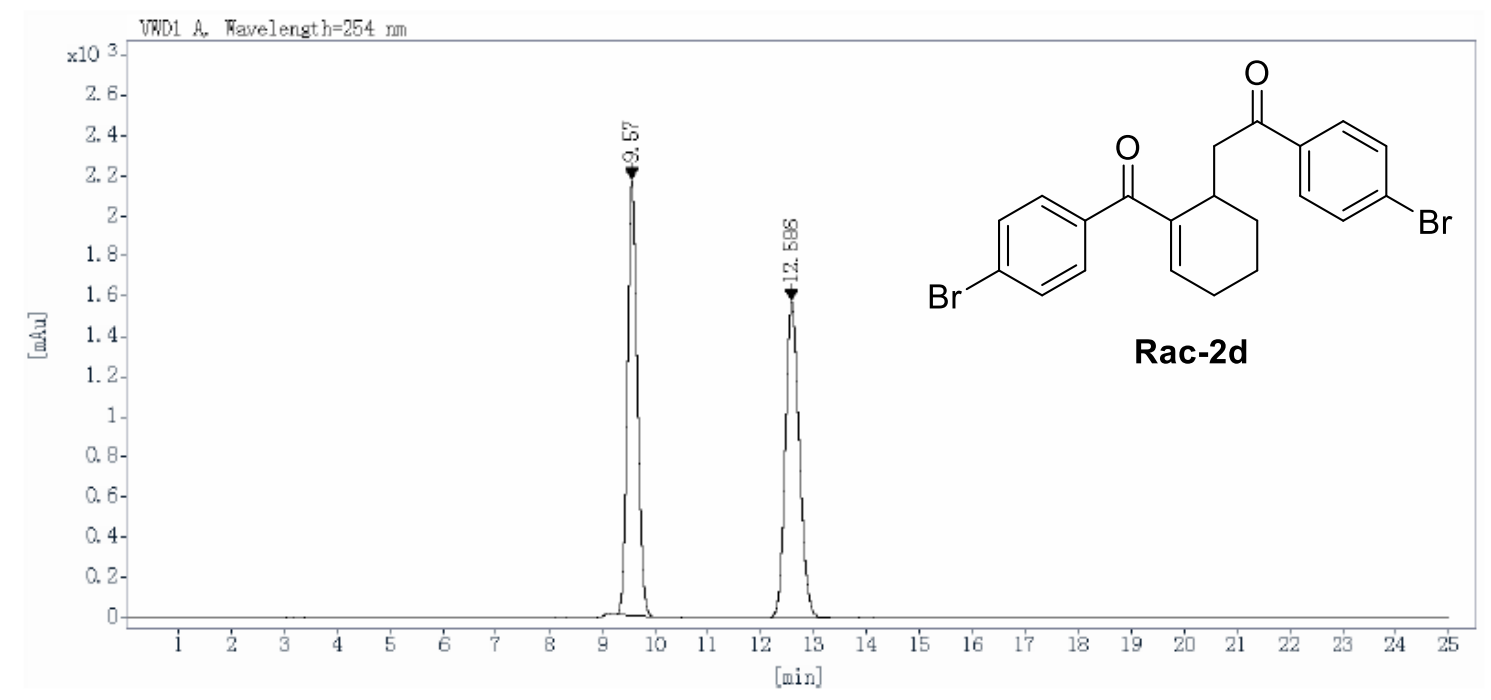

\begin{tabular}{cccccc}
$\begin{array}{c}\text { Ret Time } \\
\text { [min] }\end{array}$ & $\begin{array}{c}\text { Peak } \\
\text { Type }\end{array}$ & $\begin{array}{c}\text { Width } \\
{[\mathrm{min}]}\end{array}$ & $\begin{array}{c}\text { Height } \\
{[\mathrm{mAU}]}\end{array}$ & $\begin{array}{c}\text { Area } \\
{\left[\mathrm{mAU}^{*} \text { s] }\right.}\end{array}$ & $\begin{array}{c}\text { Area } \\
{[\%]}\end{array}$ \\
\hline 9.570 & BB & 0.21 & 2170.3972 & 28850.8203 & 49.8357 \\
12.586 & BB & 0.29 & $\begin{array}{c}1576.6456 \\
\text { Totals: }\end{array}$ & $\begin{array}{c}29041.0801 \\
57891.9004\end{array}$ & 50.1643 \\
& & & 100.0000
\end{tabular}

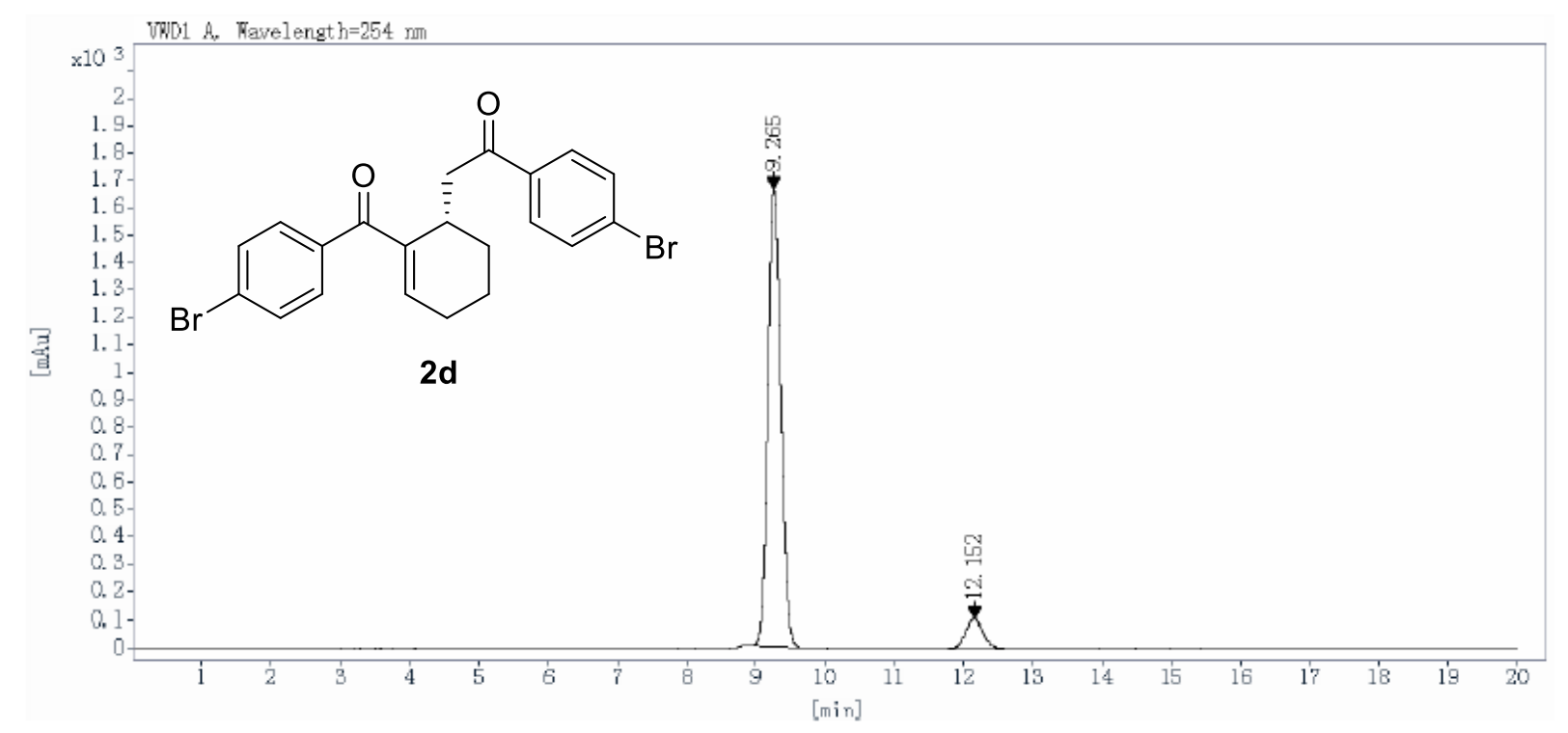

$\begin{array}{cccccc}\begin{array}{c}\text { Ret Time } \\ \text { [min] }\end{array} & \begin{array}{c}\text { Peak } \\ \text { Type }\end{array} & \begin{array}{c}\text { Width } \\ \text { [min] }\end{array} & \begin{array}{c}\text { Height } \\ \text { [mAU] }\end{array} & \begin{array}{c}\text { Area } \\ {[\mathbf{m A U} \text { *s] }}\end{array} & \begin{array}{c}\text { Area } \\ {[\%]}\end{array} \\ 9.265 & \text { VB R } & 0.20 & 1657.4457 & 21362.3145 & 92.1490 \\ 12.152 & \text { BB } & 0.28 & \begin{array}{c}102.2415 \\ \text { Totals: }\end{array} & 231820.0372 & 7.8510 \\ & & & & & \\ & & & & & \end{array}$


<smiles>O=C(C[C@@H]1CCCC=C1C(=O)c1cccc(Br)c1)c1cccc(Br)c1</smiles>

$2 e$

${ }^{1} \mathrm{H}$ NMR $\left(400 \mathrm{MHz}, \mathrm{CDCl}_{3}\right)$

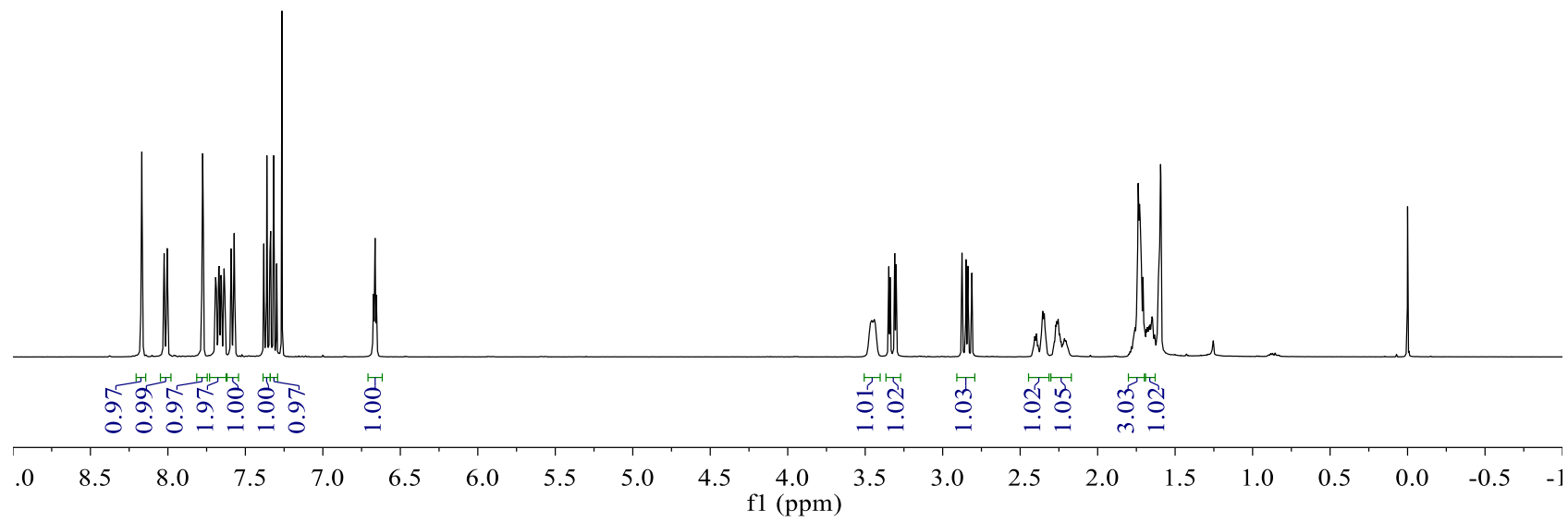

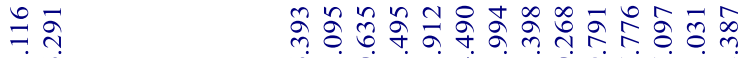

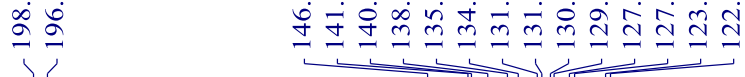<smiles>O=C(C[C@@H]1CCCC=C1C(=O)c1cccc(Br)c1)c1cccc(Br)c1</smiles>

2e

${ }^{13} \mathrm{C}$ NMR $\left(100 \mathrm{MHz}, \mathrm{CDCl}_{3}\right)$

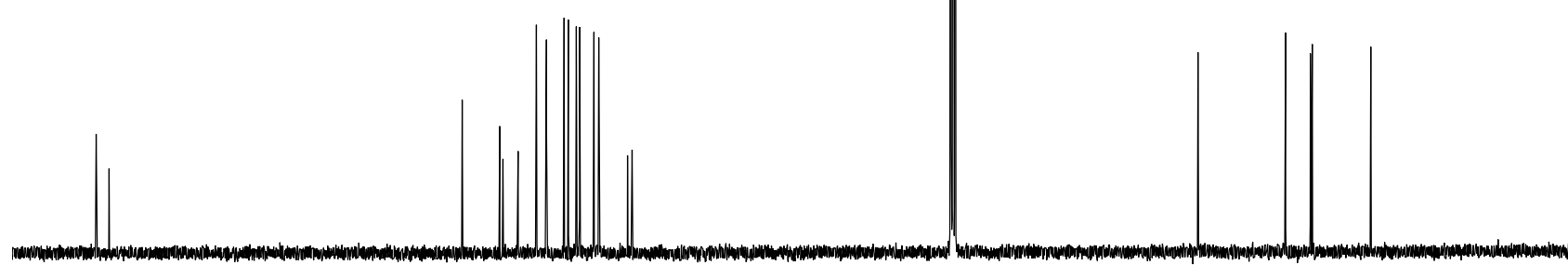

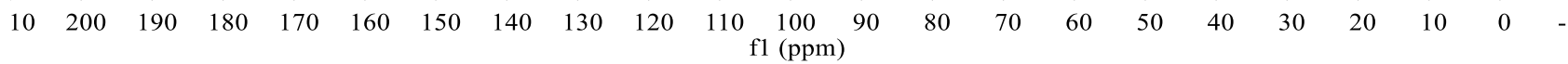




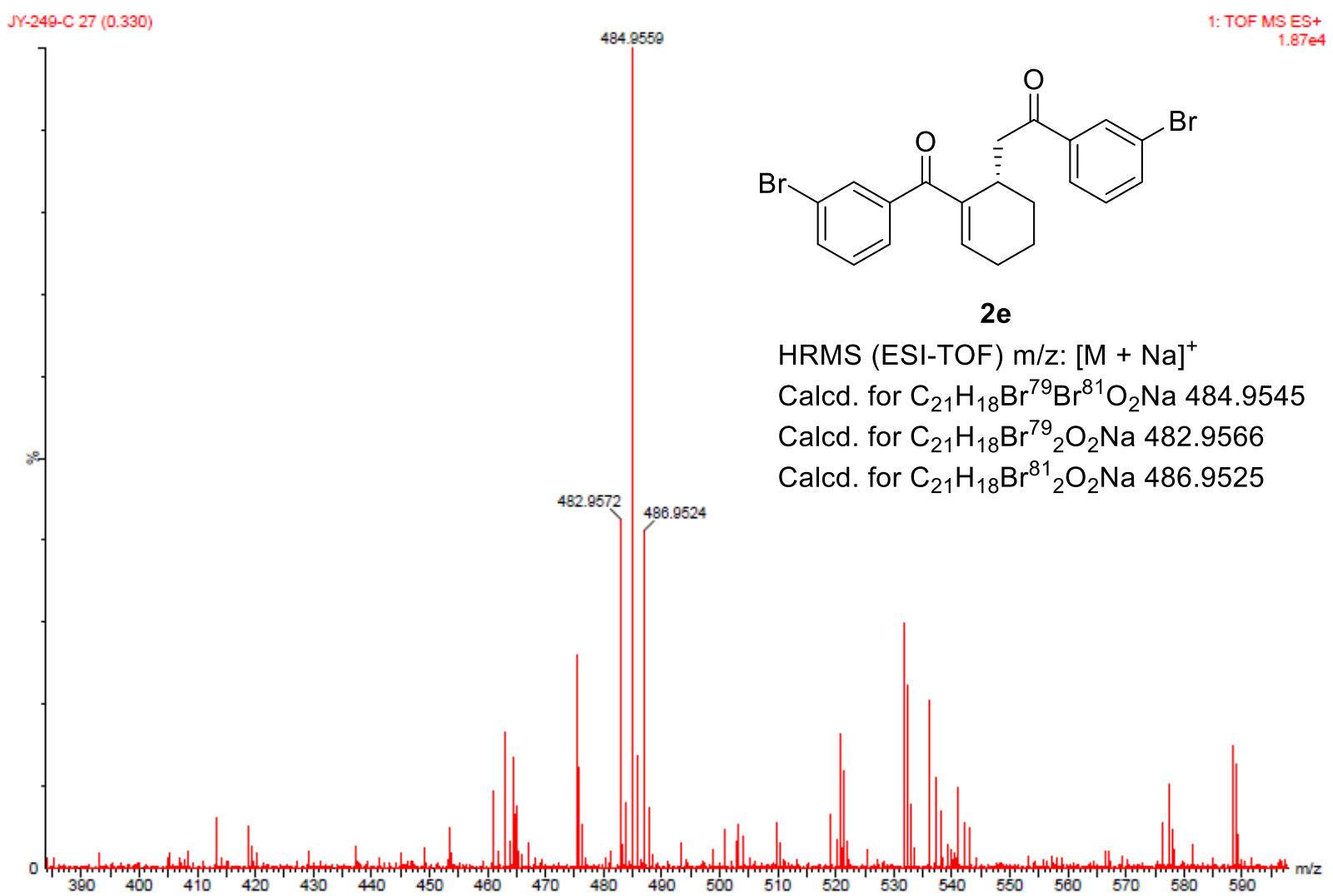




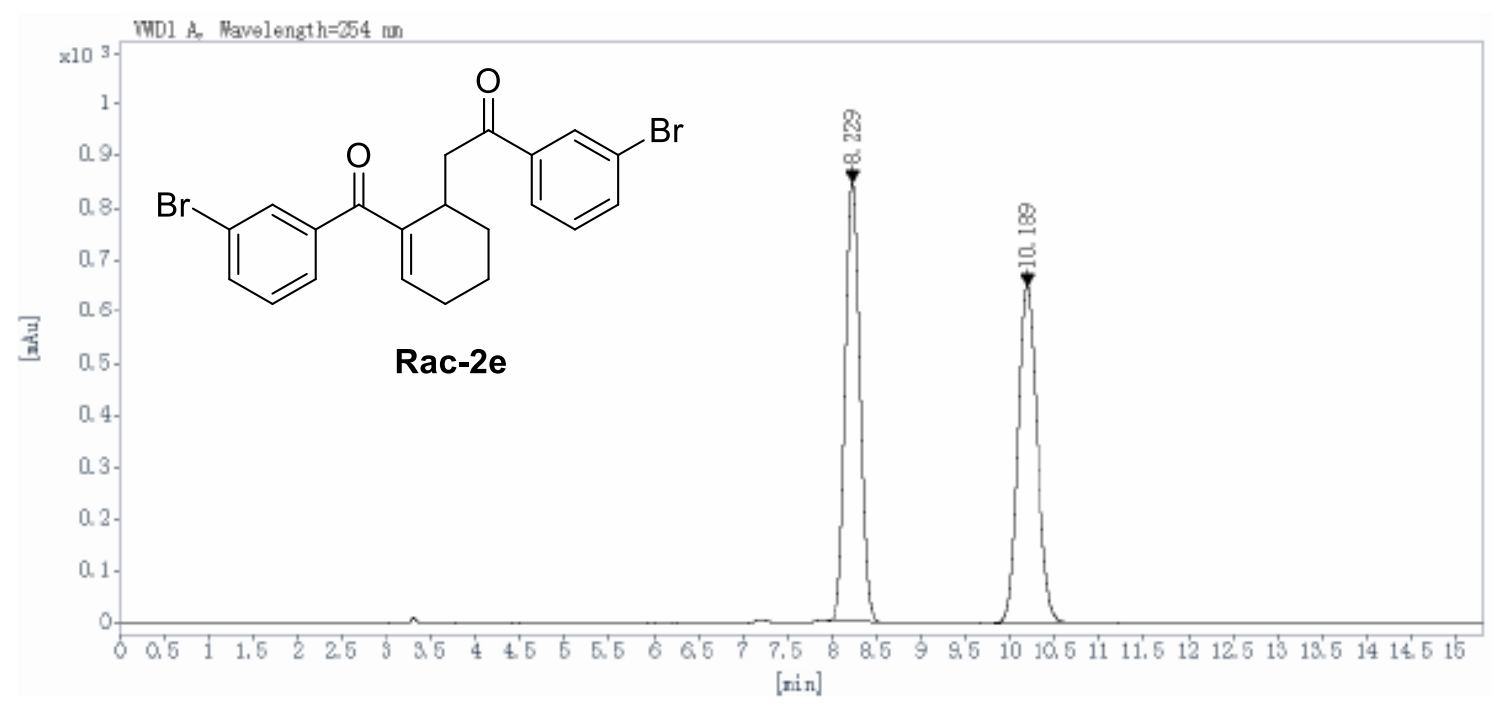

\begin{tabular}{cccccc}
$\begin{array}{c}\text { Ret Time } \\
\text { [min] }\end{array}$ & $\begin{array}{c}\text { Peak } \\
\text { Type }\end{array}$ & $\begin{array}{c}\text { Width } \\
{[\mathrm{min}]}\end{array}$ & $\begin{array}{c}\text { Height } \\
{[\mathrm{mAU}]}\end{array}$ & $\begin{array}{c}\text { Area } \\
{\left[\mathrm{mAU}^{*} \text { s] }\right.}\end{array}$ & $\begin{array}{c}\text { Area } \\
{[\%]}\end{array}$ \\
\hline 8.229 & VB R & 0.17 & 846.2742 & 9495.6133 & 49.6795 \\
10.189 & BB & 0.23 & 650.0742 & 9618.1250 & 50.3205 \\
& & & Totals: & 19113.7383 & 100.0000
\end{tabular}

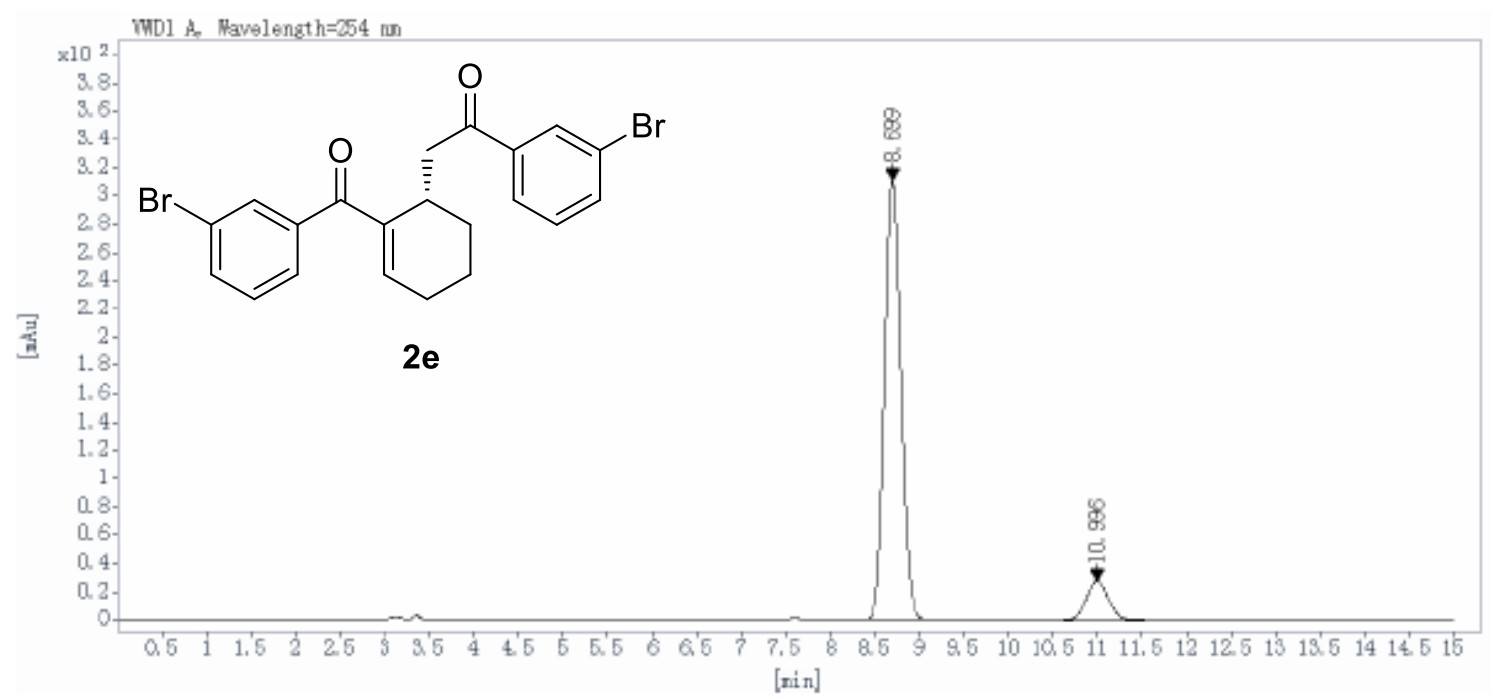

\begin{tabular}{cccccc}
$\begin{array}{c}\text { Ret Time } \\
\text { [min] }\end{array}$ & $\begin{array}{c}\text { Peak } \\
\text { Type }\end{array}$ & $\begin{array}{c}\text { Width } \\
{[\mathrm{min}]}\end{array}$ & $\begin{array}{c}\text { Height } \\
{[\mathrm{mAU}]}\end{array}$ & $\begin{array}{c}\text { Area } \\
{\left[\mathrm{mAU}^{*} \text { s] }\right.}\end{array}$ & $\begin{array}{c}\text { Area } \\
{[\%]}\end{array}$ \\
\hline 8.699 & BB & 0.20 & 310.9639 & 3909.9094 & 89.3063 \\
10.996 & BB & 0.26 & $\begin{array}{c}27.6277 \\
\text { Totals: }\end{array}$ & $\begin{array}{c}468.1816 \\
4378.0910\end{array}$ & 10.6937 \\
& & & 100.0000
\end{tabular}


<smiles>O=C(C[C@@H]1CCCC=C1C(=O)c1ccc2ccccc2c1)c1ccc2ccccc2c1</smiles>

$2 f$

${ }^{1} \mathrm{H} \operatorname{NMR}\left(400 \mathrm{MHz}, \mathrm{CDCl}_{3}\right)$

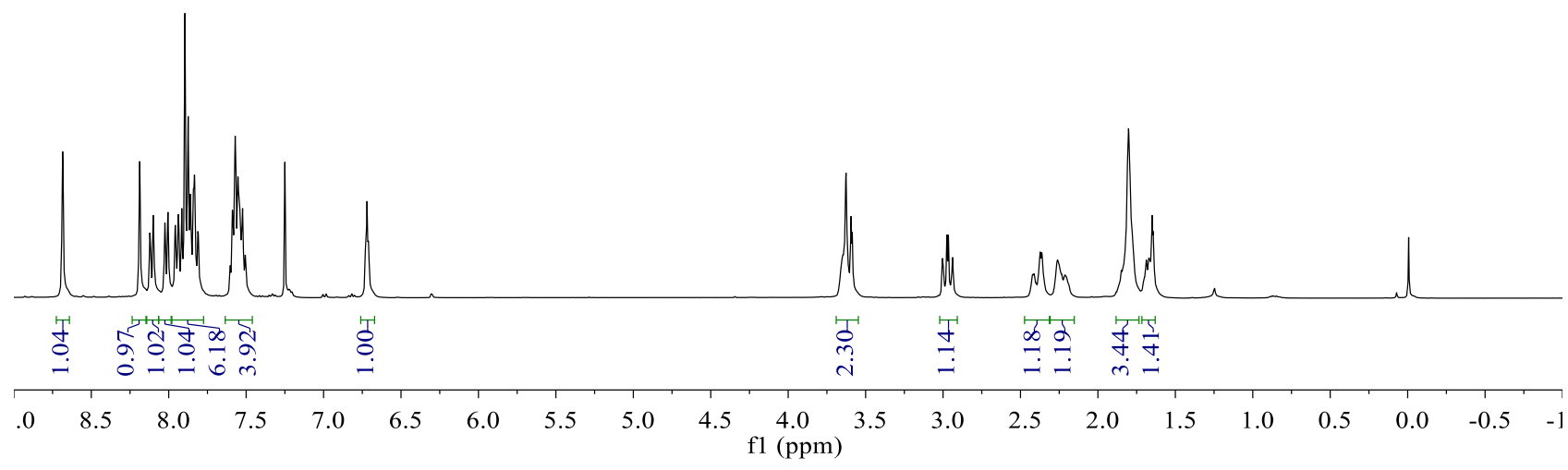

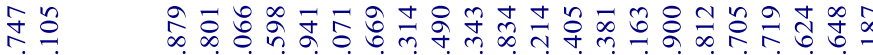

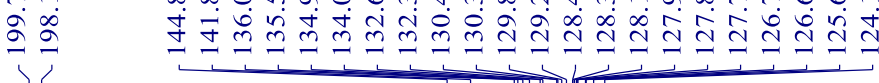

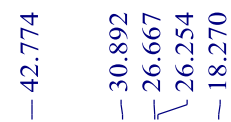<smiles>O=C(C[C@@H]1CCCC=C1C(=O)c1ccc2ccccc2c1)c1ccc2ccccc2c1</smiles>

$2 f$

${ }^{13} \mathrm{C}$ NMR $\left(100 \mathrm{MHz}, \mathrm{CDCl}_{3}\right)$

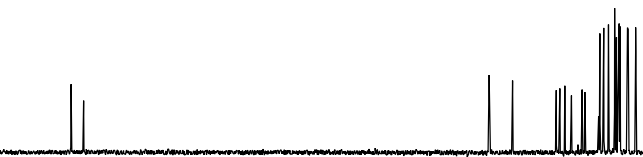

$\begin{array}{lllllllllllllllllllllllll}10 & 200 & 190 & 180 & 170 & 160 & 150 & 140 & 130 & 120 & 110 & 100 & 90 & 80 & 70 & 60 & 50 & 40 & 30 & 20 & 10 & 0 & -\end{array}$ 


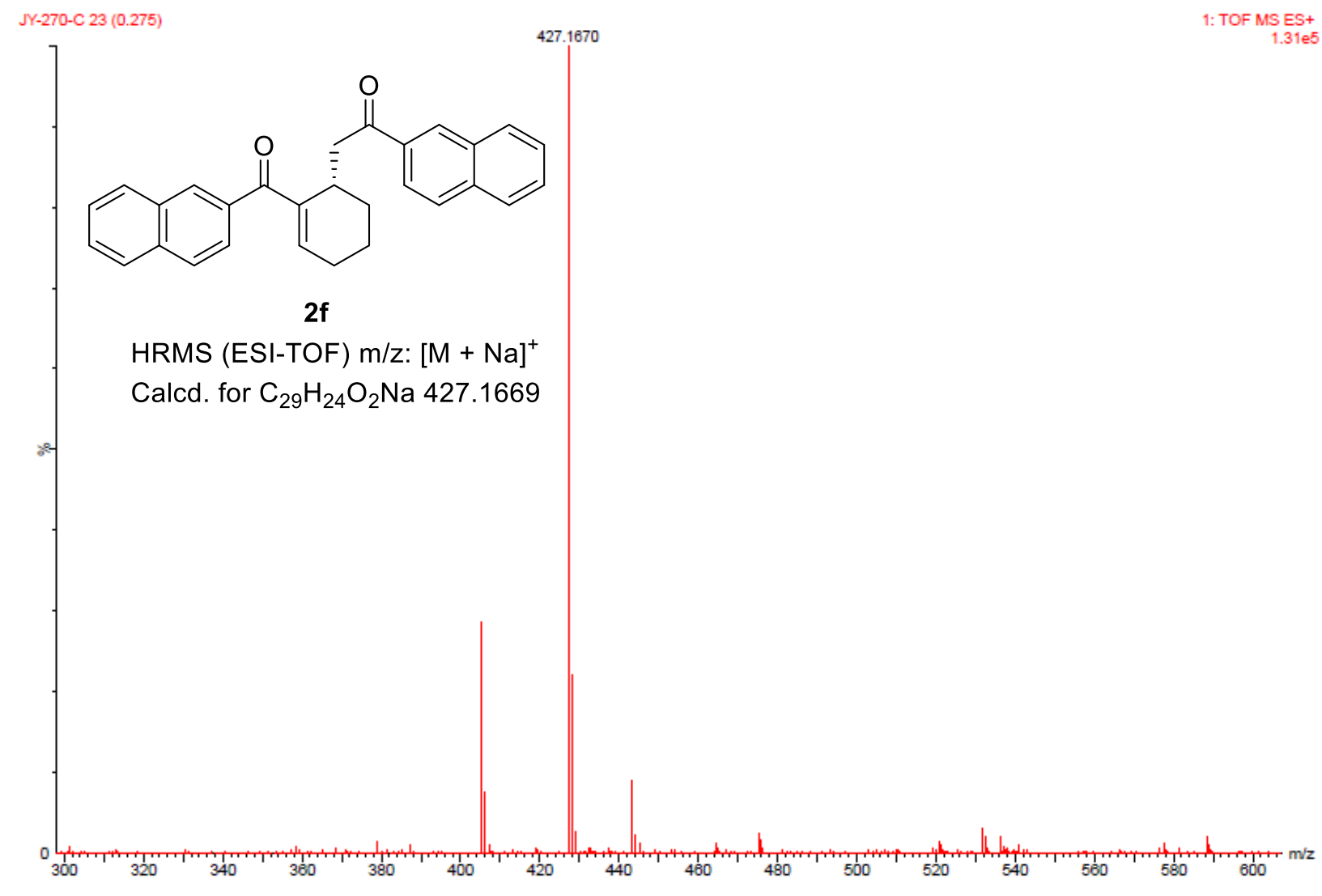




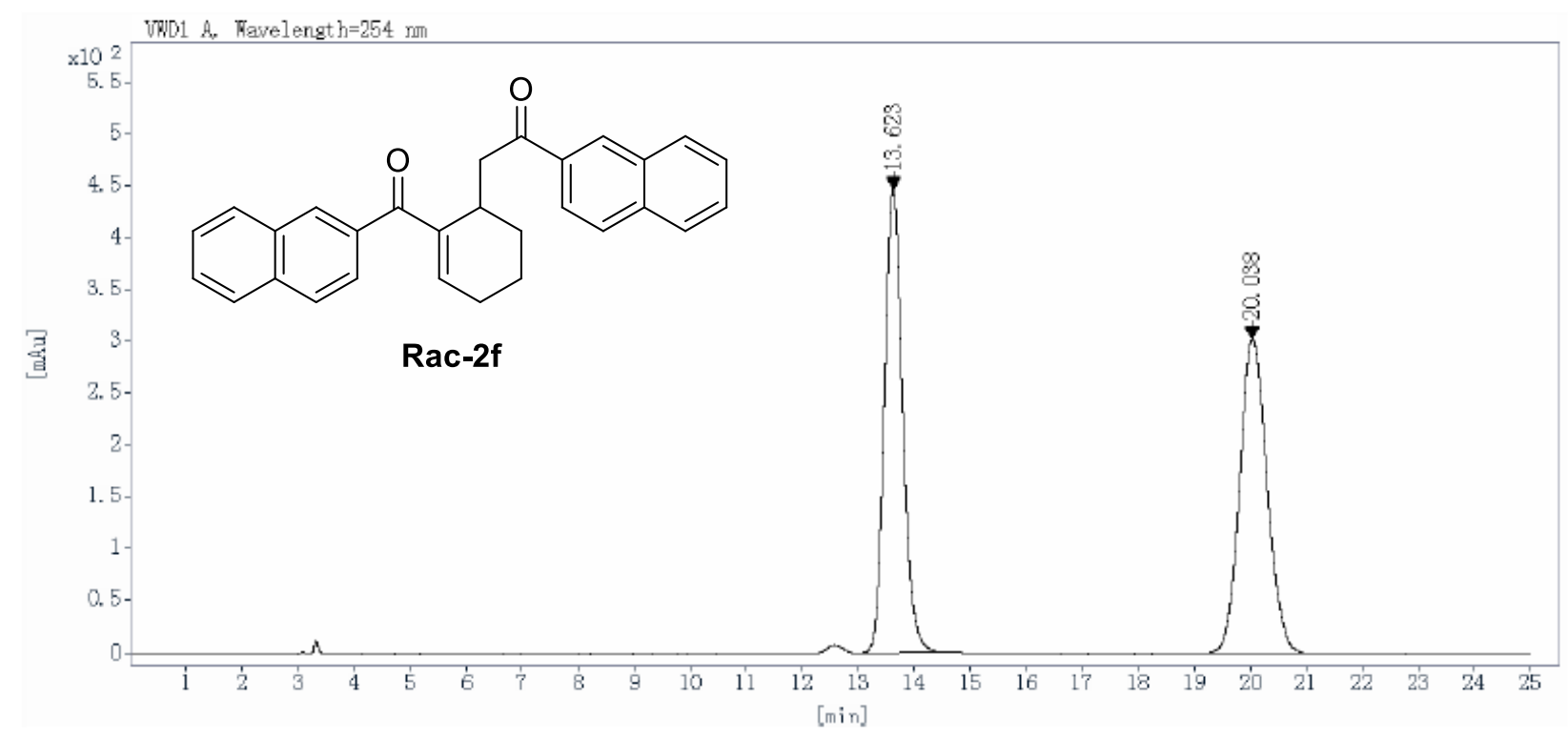

$\begin{array}{cccccc}\begin{array}{c}\text { Ret Time } \\ \text { [min] }\end{array} & \begin{array}{c}\text { Peak } \\ \text { Type }\end{array} & \begin{array}{c}\text { Width } \\ \text { [min] }\end{array} & \begin{array}{c}\text { Height } \\ \text { [mAU] }\end{array} & \begin{array}{c}\text { Area } \\ \text { [mAU*s] }\end{array} & \begin{array}{c}\text { Area } \\ {[\%]}\end{array} \\ 13.623 & \text { BB } & 0.35 & 445.2767 & 10013.2441 & 49.5400 \\ 20.038 & \text { BB } & 0.53 & \begin{array}{c}301.8744 \\ \text { Totals: }\end{array} & 10199.2129 & 50.4600 \\ & & & & & \end{array}$

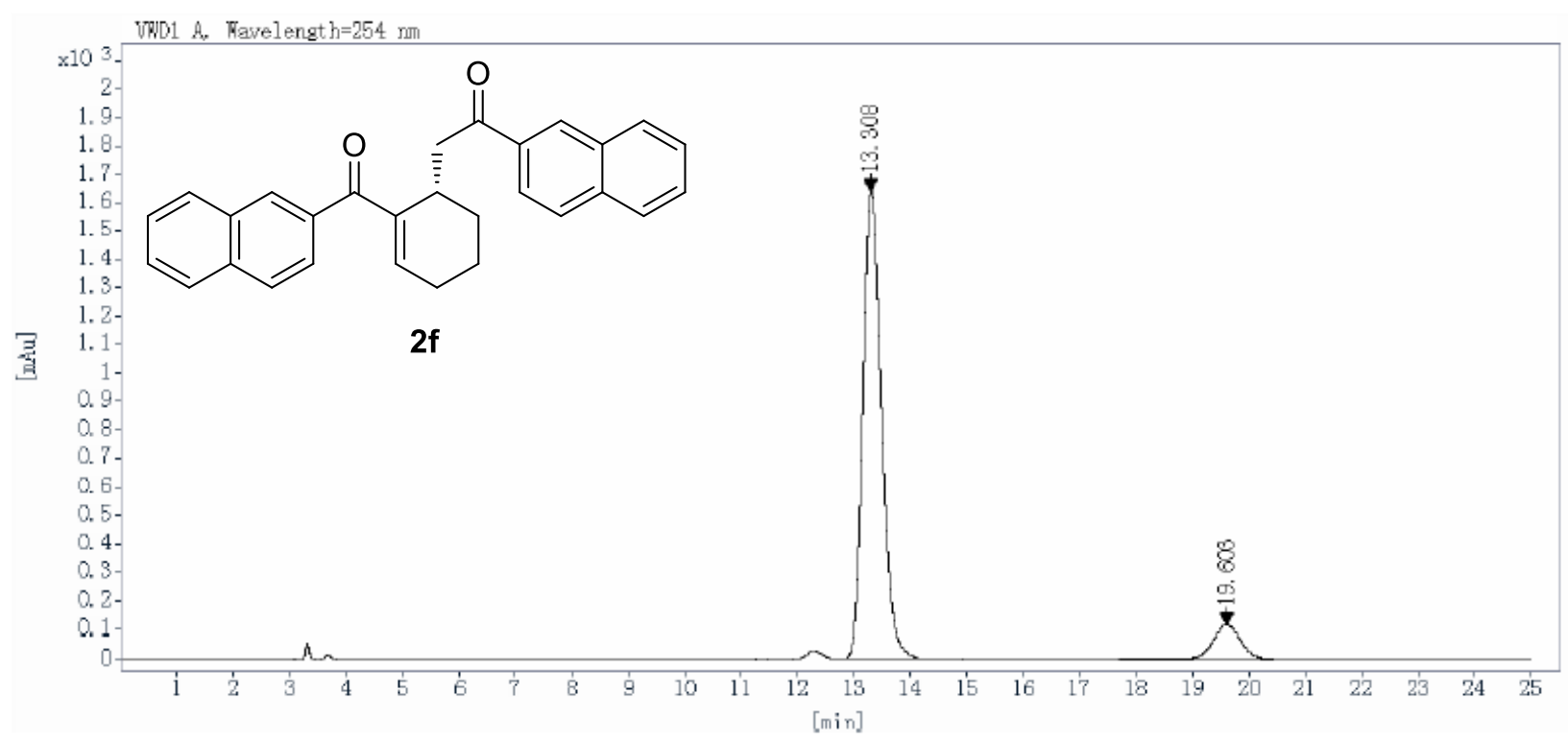

$\begin{array}{cccccc}\begin{array}{c}\text { Ret Time } \\ \text { [min] }\end{array} & \begin{array}{c}\text { Peak } \\ \text { Type }\end{array} & \begin{array}{c}\text { Width } \\ \text { [min] }\end{array} & \begin{array}{c}\text { Height } \\ \text { [mAU] }\end{array} & \begin{array}{c}\text { Area } \\ \text { [mAU*s] }\end{array} & \begin{array}{c}\text { Area } \\ {[\%]}\end{array} \\ 13.308 & \text { BB } & 0.36 & 1637.1539 & 37407.4141 & 90.2771 \\ 19.603 & \text { VB R } & 0.53 & 115.9462 & 4028.7927 & 9.7229 \\ & & & \text { Totals: } & 41436.2068 & 100.0000\end{array}$


<smiles>O=C(C[C@@H]1CCCC=C1C(=O)c1cccs1)c1cccs1</smiles>

2g

${ }^{1} \mathrm{H}$ NMR $\left(400 \mathrm{MHz}, \mathrm{CDCl}_{3}\right)$

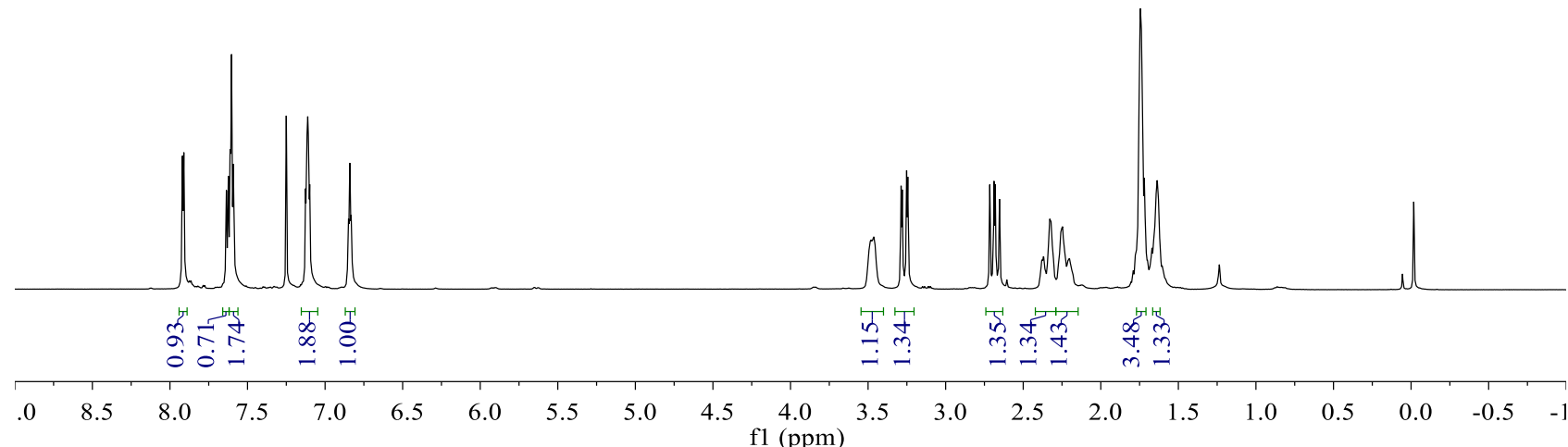

in

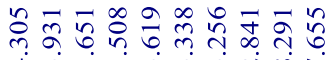

ปू

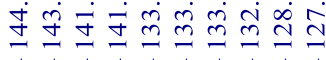<smiles>O=C(C[C@H]1CCCC=C1C(=O)c1cccs1)c1cccs1</smiles>

2g

${ }^{13} \mathrm{C} \mathrm{NMR}\left(100 \mathrm{MHz}, \mathrm{CDCl}_{3}\right)$

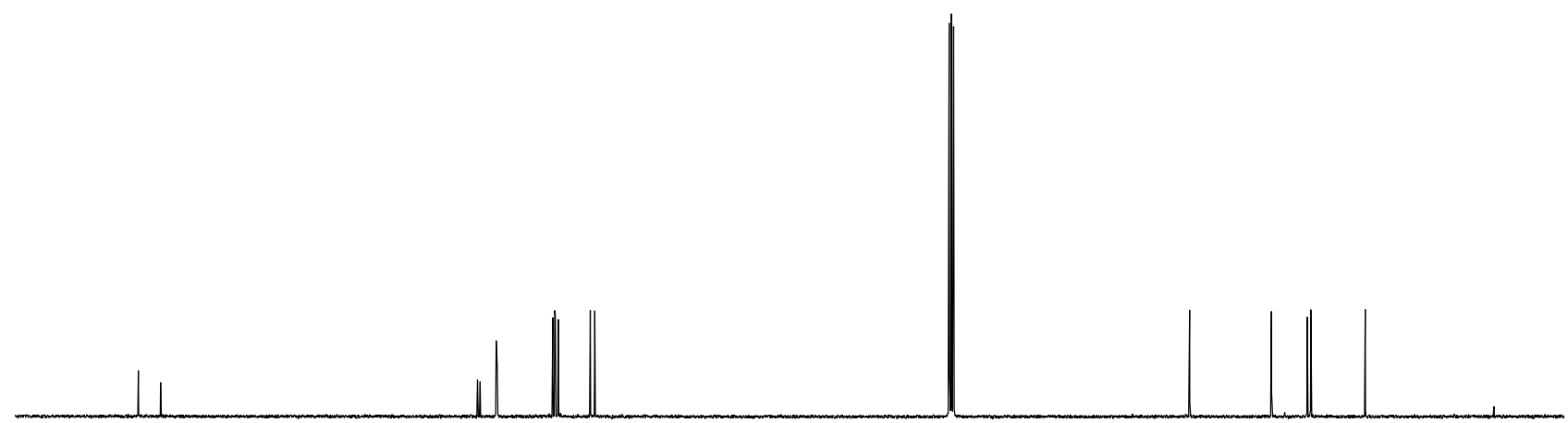

$\begin{array}{lllllllllllllllllllllll}10 & 200 & 190 & 180 & 170 & 160 & 150 & 140 & 130 & 120 & 110 & \begin{array}{c}100 \\ \mathrm{f} 1(\mathrm{ppm})\end{array}\end{array}$ 


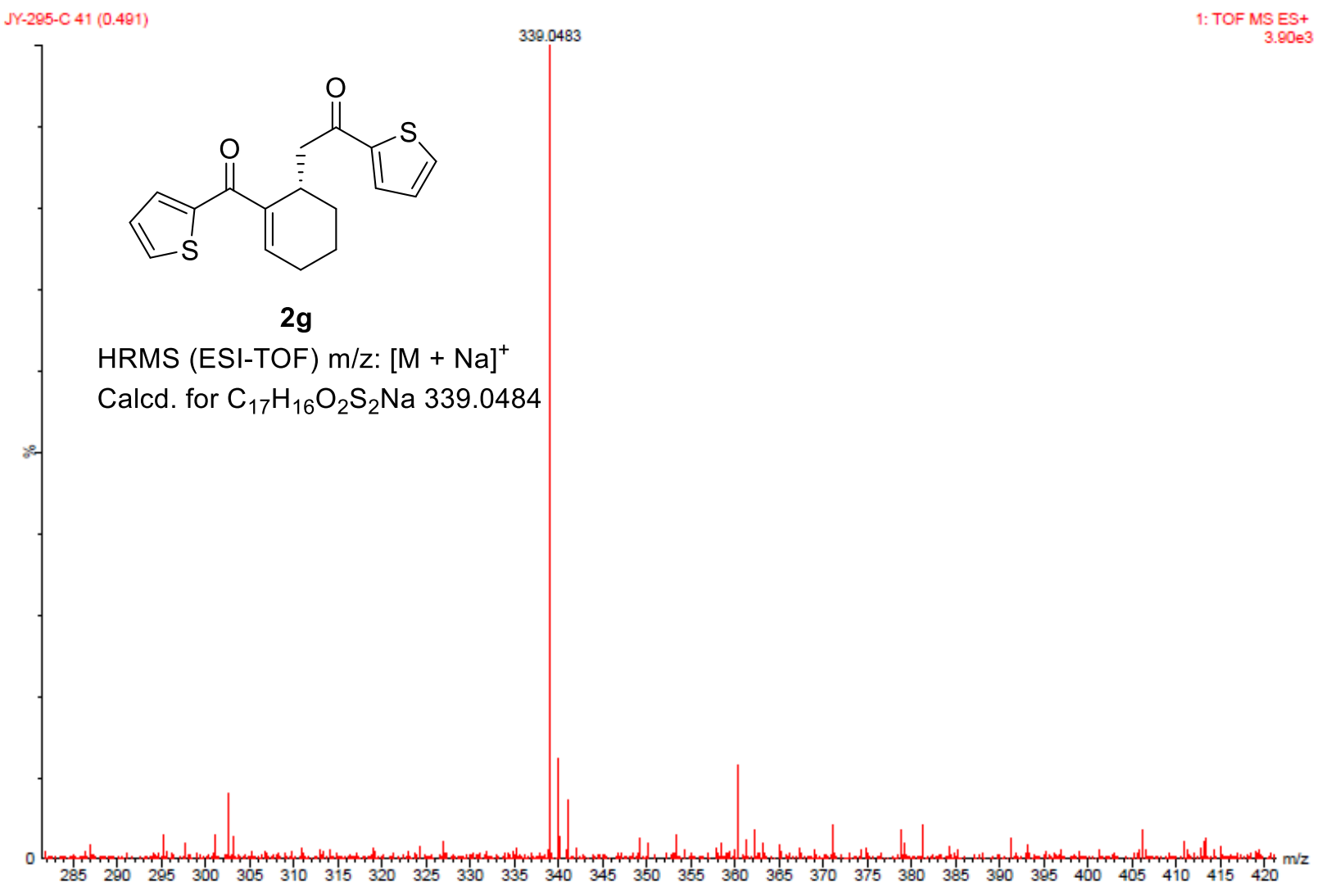




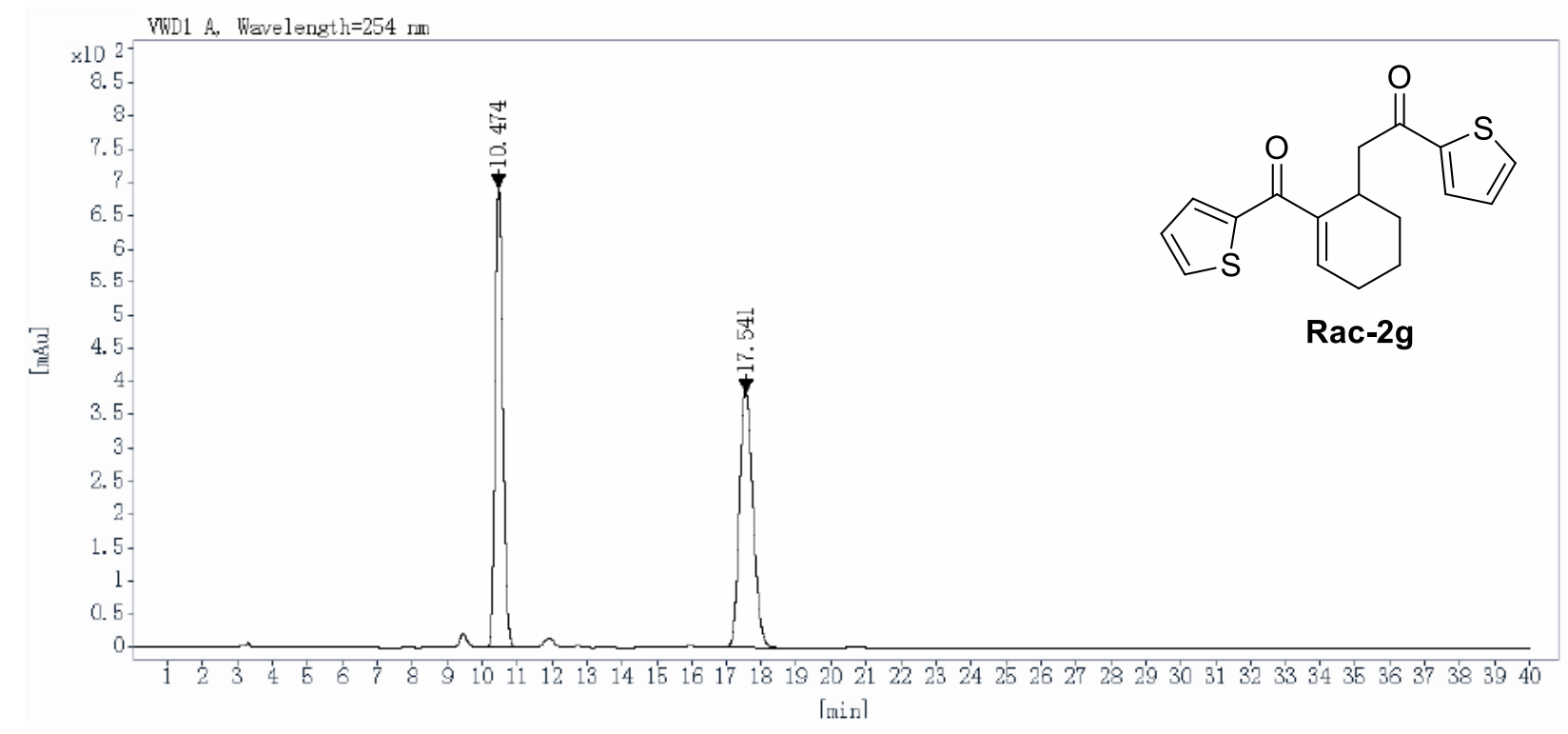

$\begin{array}{cccccc}\begin{array}{c}\text { Ret Time } \\ \text { [min] }\end{array} & \begin{array}{c}\text { Peak } \\ \text { Type }\end{array} & \begin{array}{c}\text { Width } \\ {[\mathbf{m i n}]}\end{array} & \begin{array}{c}\text { Height } \\ {[\mathbf{m A U}]}\end{array} & \begin{array}{c}\text { Area } \\ {[\mathbf{m A U} \text { *s] }}\end{array} & \begin{array}{c}\text { Area } \\ {[\%]}\end{array} \\ 10.474 & \text { VB R } & 0.23 & 692.1631 & 10374.8086 & 50.1725 \\ 17.541 & \text { BB } & 0.41 & 385.4314 & 10303.4561 & 49.8275 \\ & & & \text { Totals: } & 20678.2646 & 100.0000\end{array}$

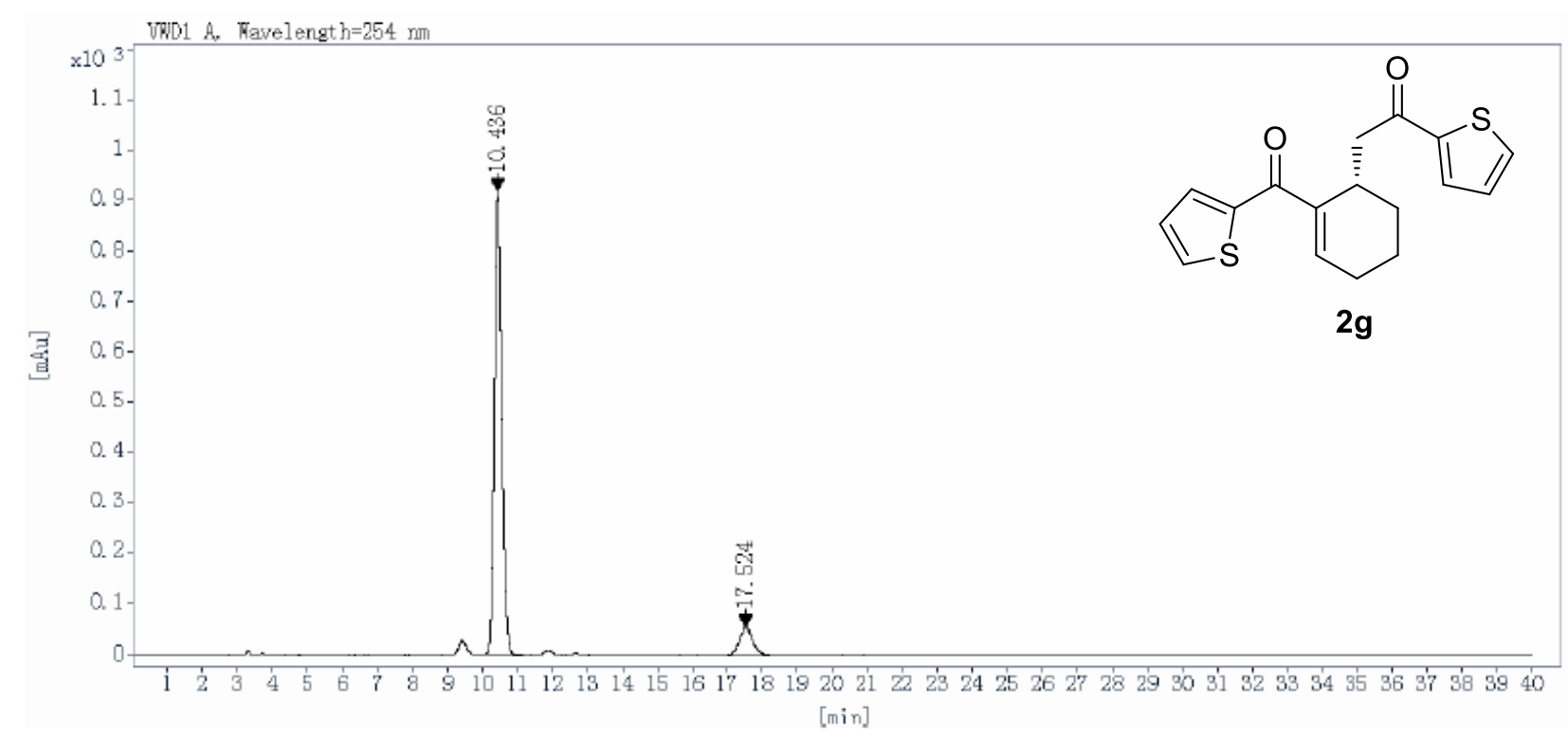

$\begin{array}{cccccc}\begin{array}{c}\text { Ret Time } \\ \text { [min] }\end{array} & \begin{array}{c}\text { Peak } \\ \text { Type }\end{array} & \begin{array}{c}\text { Width } \\ {[\mathrm{min}]}\end{array} & \begin{array}{c}\text { Height } \\ {[\mathrm{mAU}]}\end{array} & \begin{array}{c}\text { Area } \\ {\left[\mathrm{mAU}^{*} \mathbf{s}\right]}\end{array} & \begin{array}{c}\text { Area } \\ {[\%]}\end{array} \\ 10.436 & \text { VB R } & 0.23 & 916.7052 & 13803.5908 & 90.8996 \\ 17.524 & \text { BB } & 0.41 & 51.8797 & 1381.9480 & 9.1004 \\ & & & \text { Totals: } & 15185.5388 & 100.0000\end{array}$




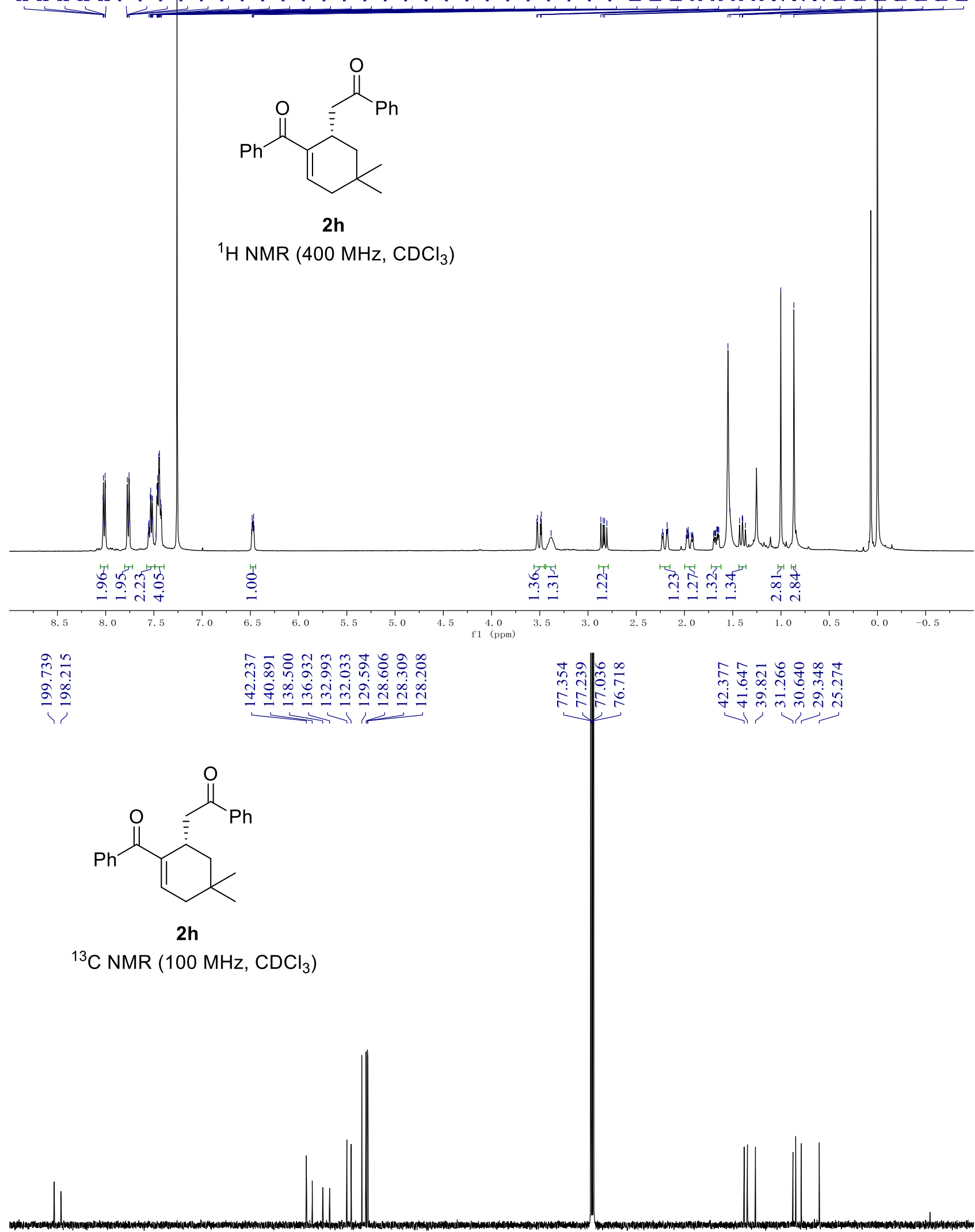

$\begin{array}{lllllllllllllllllllllll}10 & 200 & 190 & 180 & 170 & 160 & 150 & 140 & 130 & 120 & 110 & \begin{array}{c}100 \\ \mathrm{f} 1(\mathrm{ppm})\end{array} & 90 & 80 & 70 & 60 & 50 & 40 & 30 & 20 & 10 & 0 & -\end{array}$ 


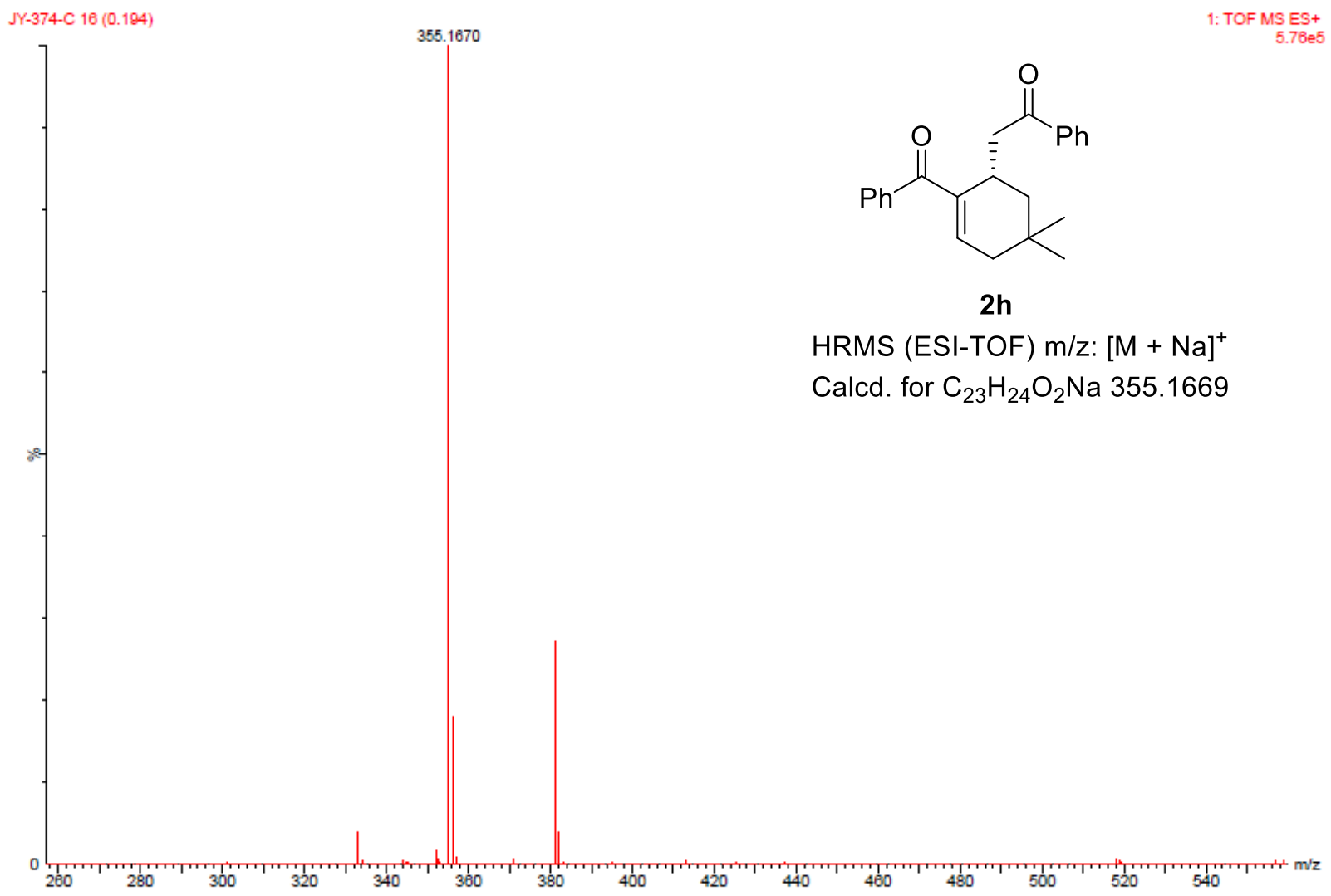




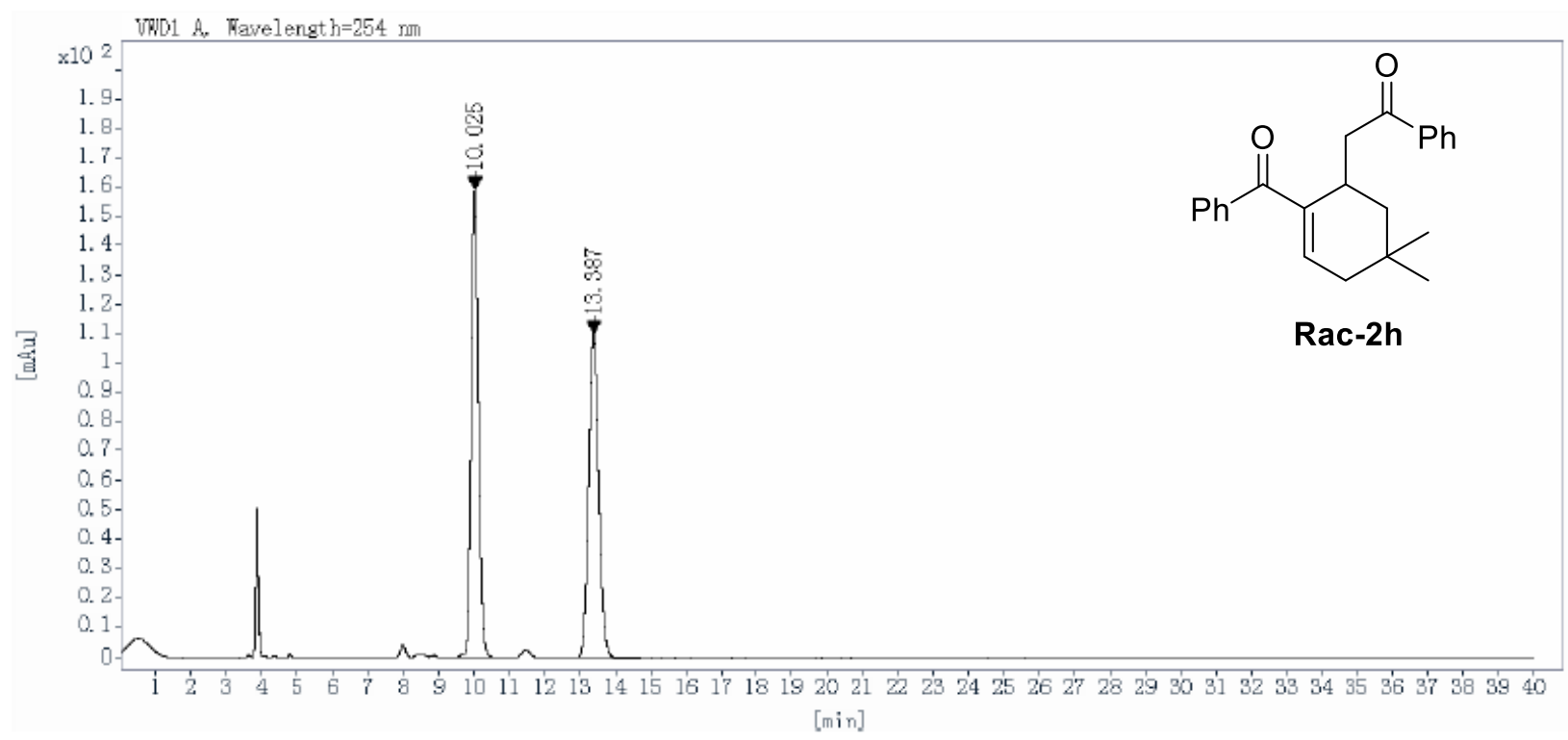

$\begin{array}{cccccc}\begin{array}{c}\text { Ret Time } \\ \text { [min] }\end{array} & \begin{array}{c}\text { Peak } \\ \text { Type }\end{array} & \begin{array}{c}\text { Width } \\ {[\mathbf{m i n}]}\end{array} & \begin{array}{c}\text { Height } \\ {[\mathbf{m A U}]}\end{array} & \begin{array}{c}\text { Area } \\ {\left[\mathbf{m A U}^{*} \text { s] }\right.}\end{array} & \begin{array}{c}\text { Area } \\ {[\%]}\end{array} \\ 10.025 & \text { BB } & 0.22 & 158.9583 & 2214.4353 & 50.1826 \\ 13.387 & \text { BB } & 0.31 & \begin{array}{c}109.7575 \\ \text { Totals: }\end{array} & 2198.3230 & 49.8174 \\ & & & & & \end{array}$

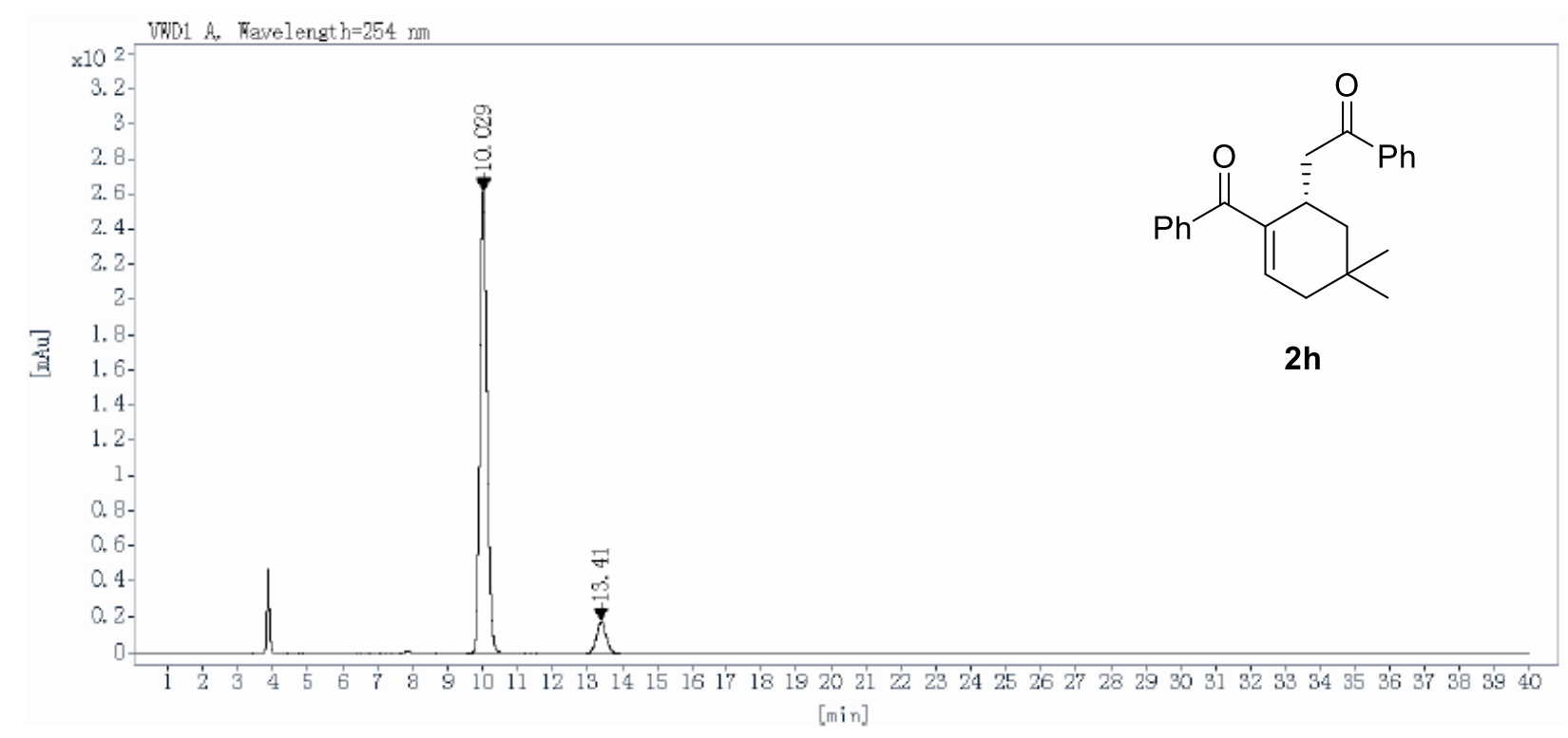

$\begin{array}{cccccc}\begin{array}{c}\text { Ret Time } \\ \text { [min] }\end{array} & \begin{array}{c}\text { Peak } \\ \text { Type }\end{array} & \begin{array}{c}\text { Width } \\ {[\mathrm{min}]}\end{array} & \begin{array}{c}\text { Height } \\ \text { [mAU] }\end{array} & \begin{array}{c}\text { Area } \\ {\left[\mathrm{mAU}^{*} \text { s] }\right.}\end{array} & \begin{array}{c}\text { Area } \\ {[\%]}\end{array} \\ 10.029 & \text { BBA } & 0.22 & 261.8331 & 3642.3843 & 91.3764 \\ 13.410 & \text { BB } & 0.31 & 17.1424 & 343.7498 & 8.6236 \\ & & & \text { Totals: } & 3986.1341 & 100.0000\end{array}$


<smiles>O=C(C[C@H]1COCC=C1C(=O)c1ccccc1)c1ccccc1</smiles>

2i

${ }^{1} \mathrm{H}$ NMR $\left(400 \mathrm{MHz}, \mathrm{CDCl}_{3}\right)$

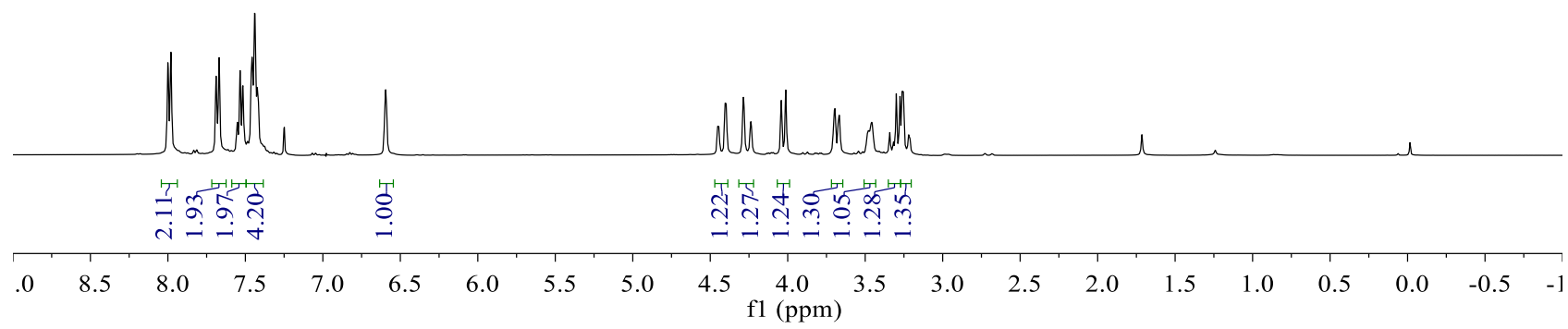

$\leqslant \frac{1}{\infty}$

文

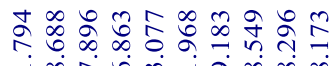

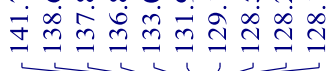

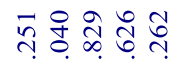

수요

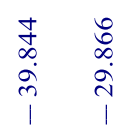<smiles>O=C(C[C@H]1COCC=C1C(=O)c1ccccc1)c1ccccc1</smiles>

2i

${ }^{13} \mathrm{C}$ NMR (150 MHz, $\mathrm{CDCl}_{3}$ )

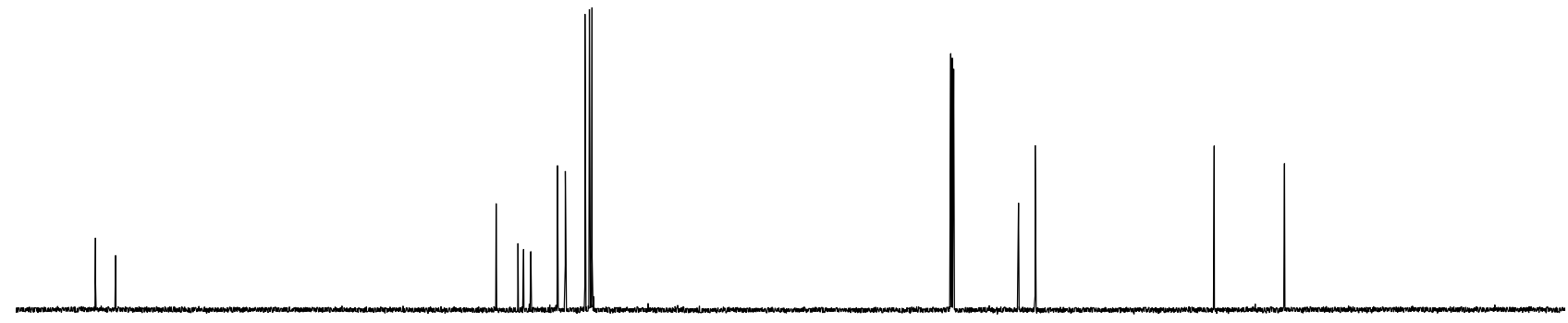

$\begin{array}{lllllllllllllllllllllll}10 & 200 & 190 & 180 & 170 & 160 & 150 & 140 & 130 & 120 & 110 \underset{\mathrm{f} 1(\mathrm{ppm})}{100} & 90 & 80 & 70 & 60 & 50 & 40 & 30 & 20 & 10 & 0 & -\end{array}$ 


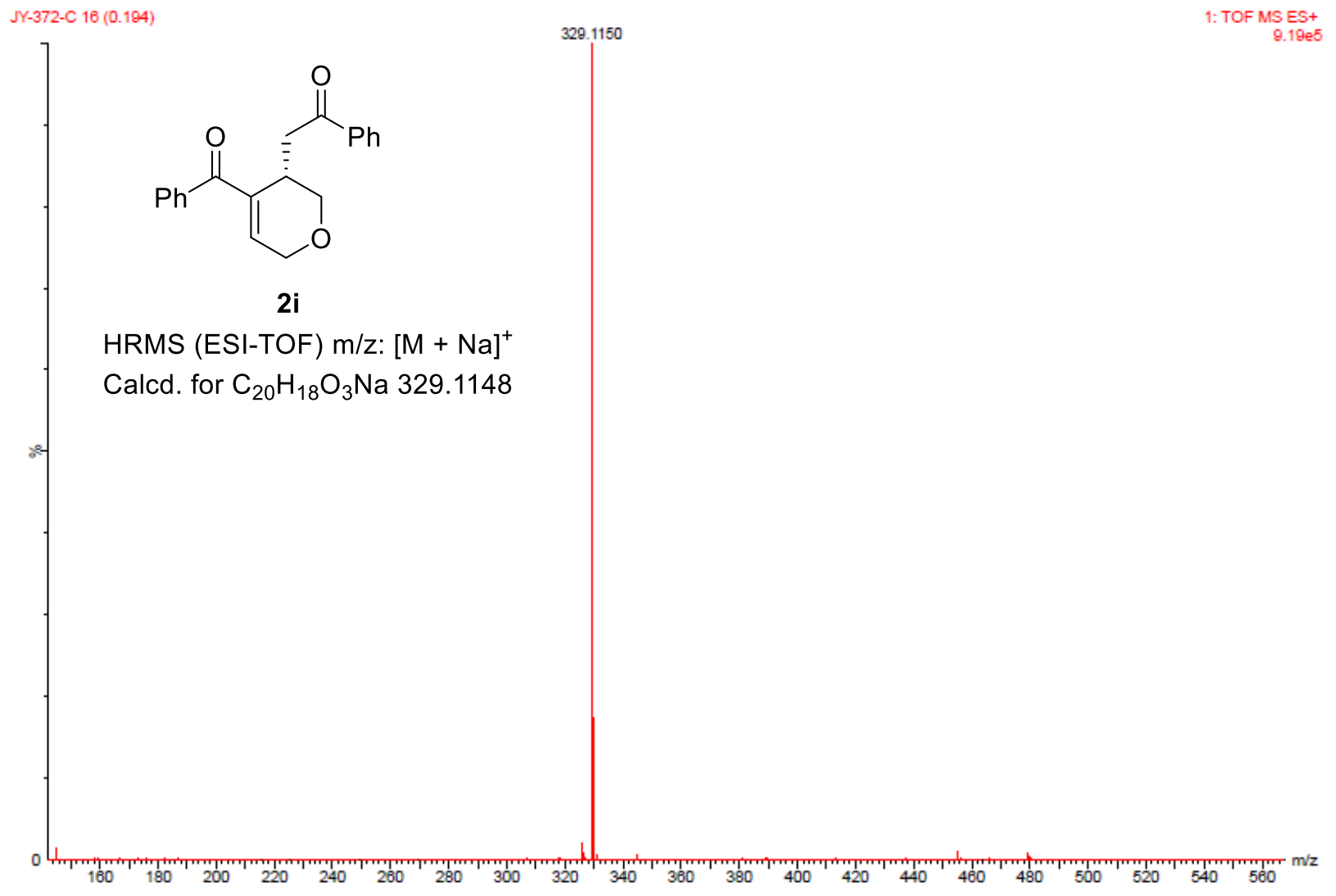




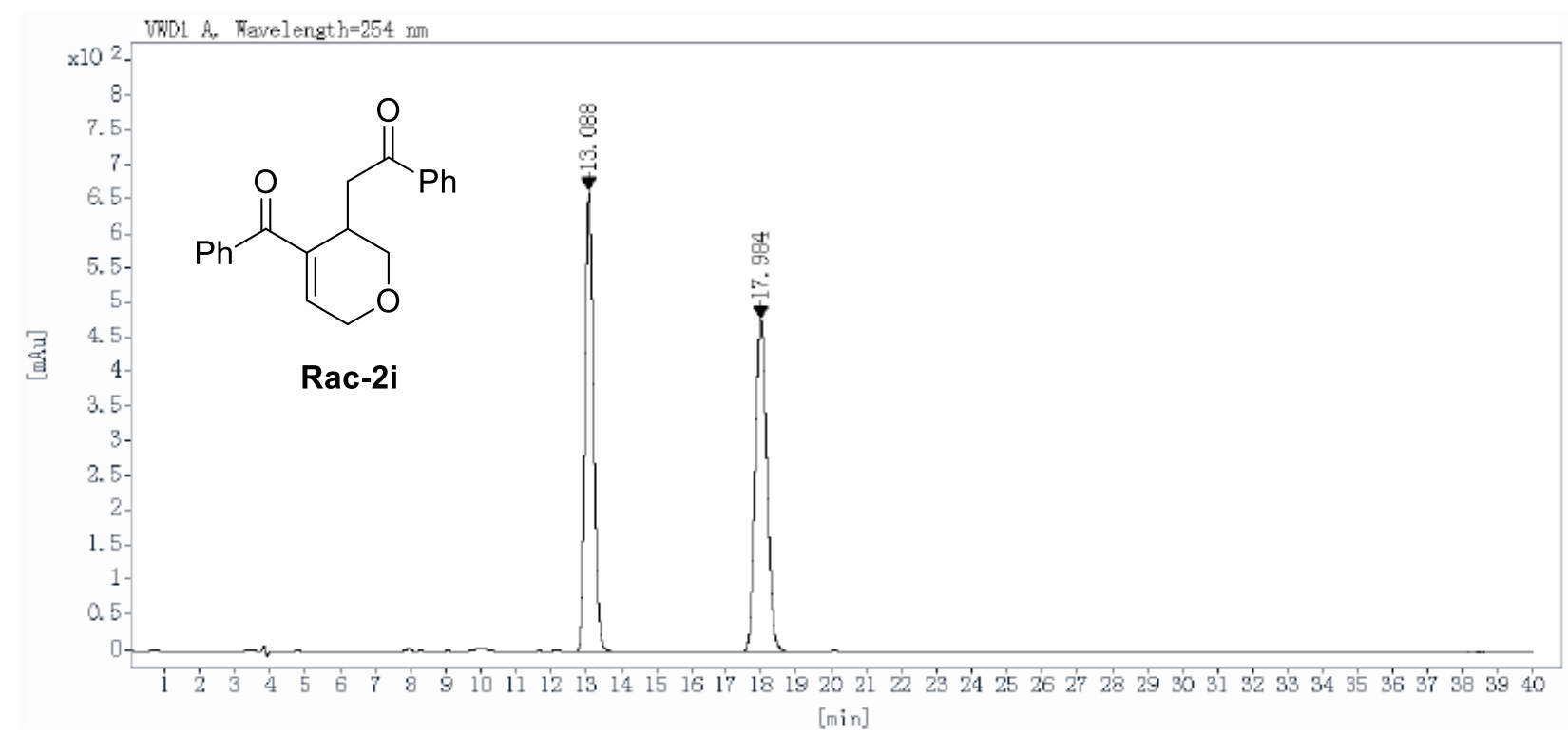

$\begin{array}{cccccc}\begin{array}{c}\text { Ret Time } \\ \text { [min] }\end{array} & \begin{array}{c}\text { Peak } \\ \text { Type }\end{array} & \begin{array}{c}\text { Width } \\ \text { [min] }\end{array} & \begin{array}{c}\text { Height } \\ \text { [mAU] }\end{array} & \begin{array}{c}\text { Area } \\ {\left[\mathbf{m A U}^{*} \text { s] }\right.}\end{array} & \begin{array}{c}\text { Area } \\ {[\%]}\end{array} \\ 13.088 & \text { BB } & 0.26 & 663.7944 & 10936.5713 & 49.9232 \\ 17.984 & \text { BB } & 0.35 & 479.4013 & 10970.2178 & 50.0768 \\ & & & \text { Totals: } & 21906.7891 & 100.0000\end{array}$

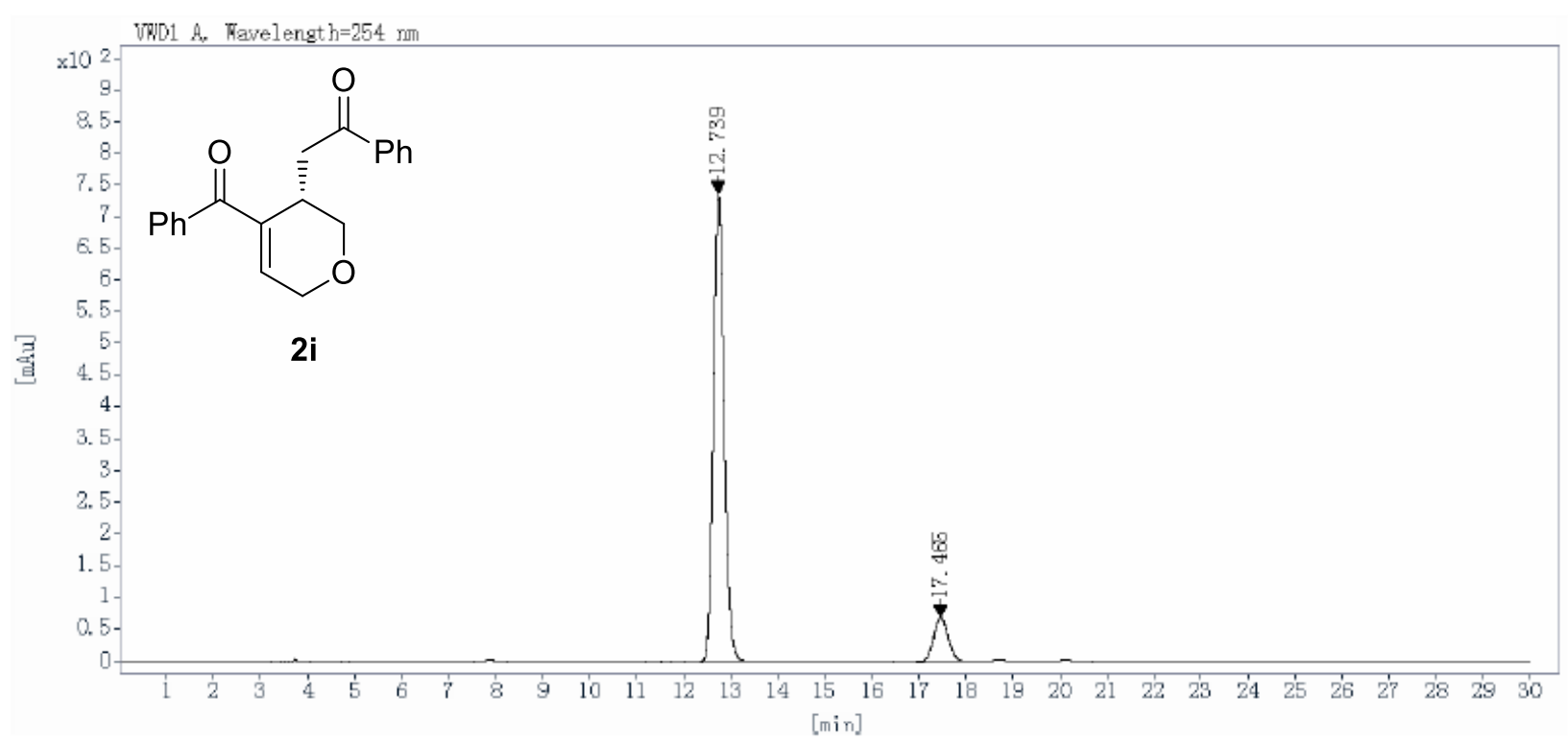

$\begin{array}{cccccc}\begin{array}{c}\text { Ret Time } \\ \text { [min] }\end{array} & \begin{array}{c}\text { Peak } \\ \text { Type }\end{array} & \begin{array}{c}\text { Width } \\ \text { [min] }\end{array} & \begin{array}{c}\text { Height } \\ \text { [mAU] }\end{array} & \begin{array}{c}\text { Area } \\ {\left[\mathbf{m A U}^{*} \text { s] }\right.}\end{array} & \begin{array}{c}\text { Area } \\ {[\%]}\end{array} \\ 12.739 & \text { BB } & 0.25 & 735.8825 & 11776.3623 & 88.9311 \\ 17.465 & \text { BB } & 0.34 & 66.8987 & 1465.7587 & 11.0689 \\ & & & \text { Totals: } & 13242.1210 & 100.0000\end{array}$


<smiles>Cc1ccc(C(=O)C[C@H]2CCCC=C2C(=O)c2ccc([N+](=O)[O-])cc2)cc1</smiles>

2j

${ }^{1} \mathrm{H}$ NMR $\left(400 \mathrm{MHz}, \mathrm{CDCl}_{3}\right)$

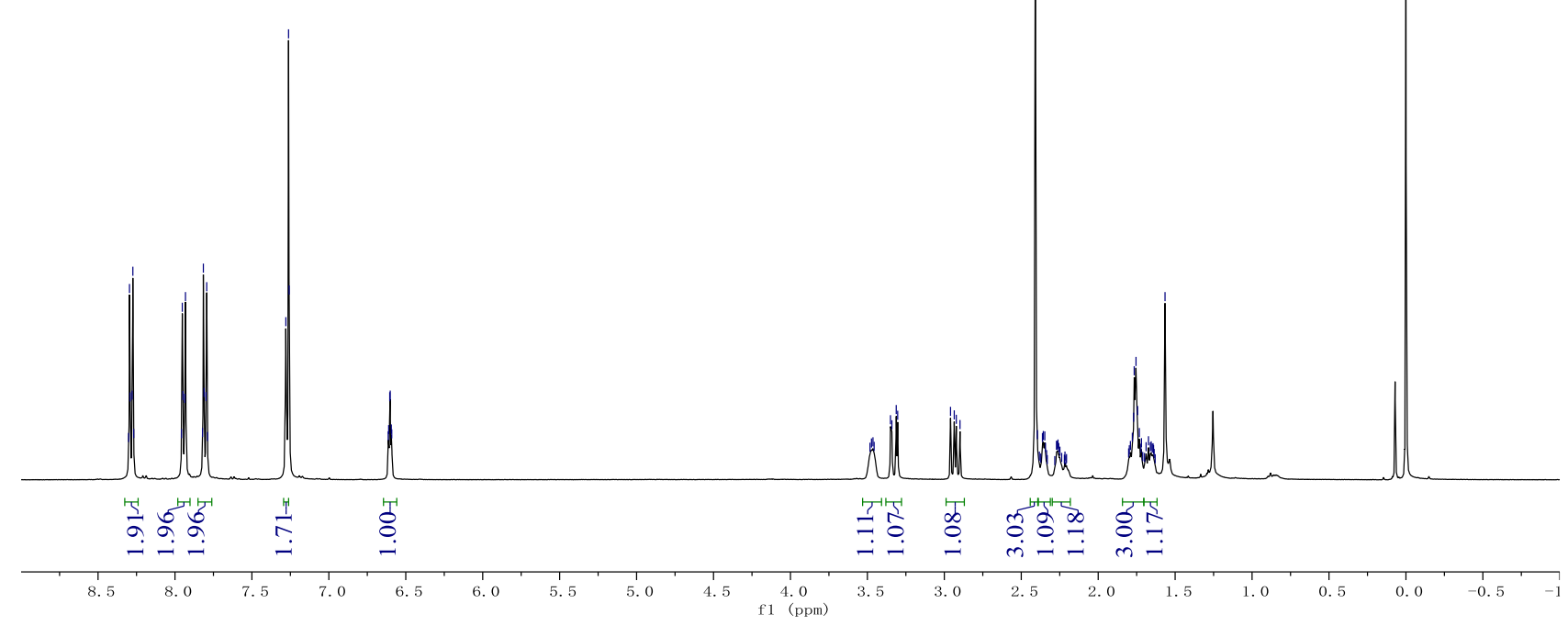

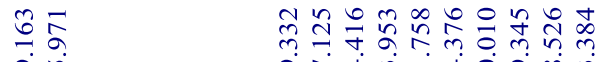

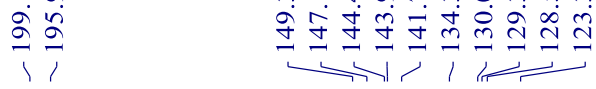<smiles>Cc1ccc(C(=O)C[C@H]2CCCC=C2C(=O)c2ccc([N+](=O)[O-])cc2)cc1</smiles>

2j

${ }^{13} \mathrm{C} \mathrm{NMR}\left(100 \mathrm{MHz}, \mathrm{CDCl}_{3}\right)$

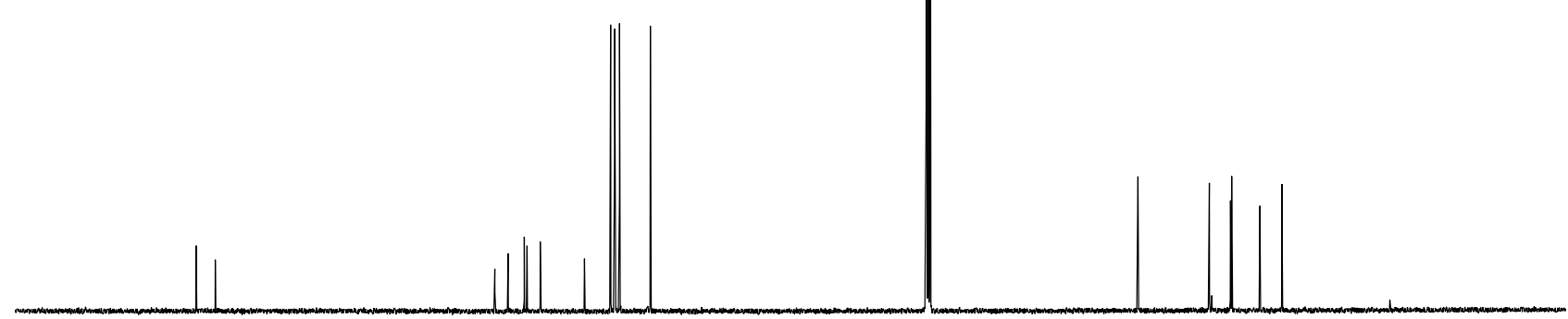

$\begin{array}{llllllllllllllllllllllllll}220 & 210 & 200 & 190 & 180 & 170 & 160 & 150 & 140 & 130 & 120 & 110 & 100 & 90 & 80 & 70 & 60 & 50 & 40 & 30 & 20 & 10 & 0 & -10 & -20\end{array}$ 


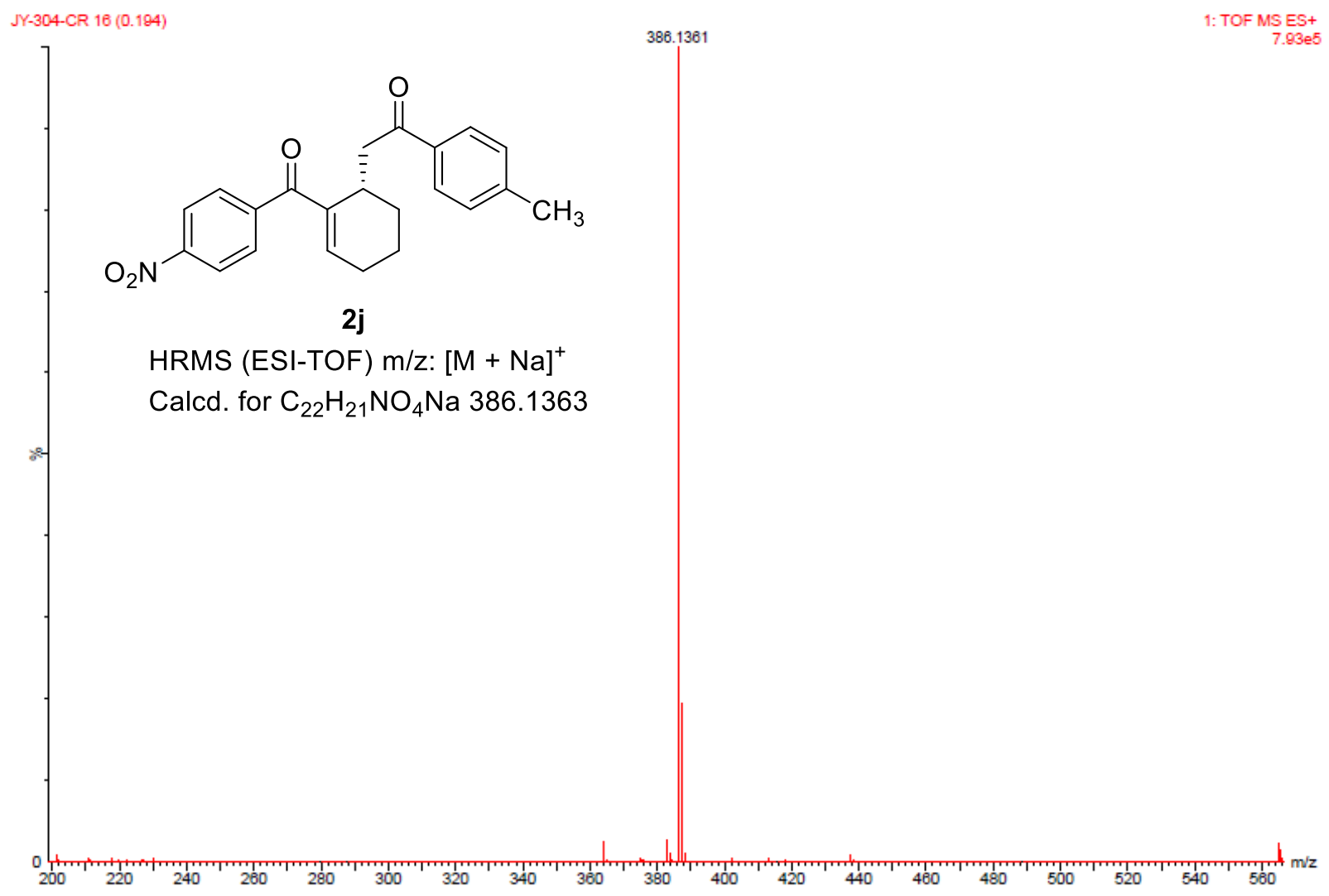




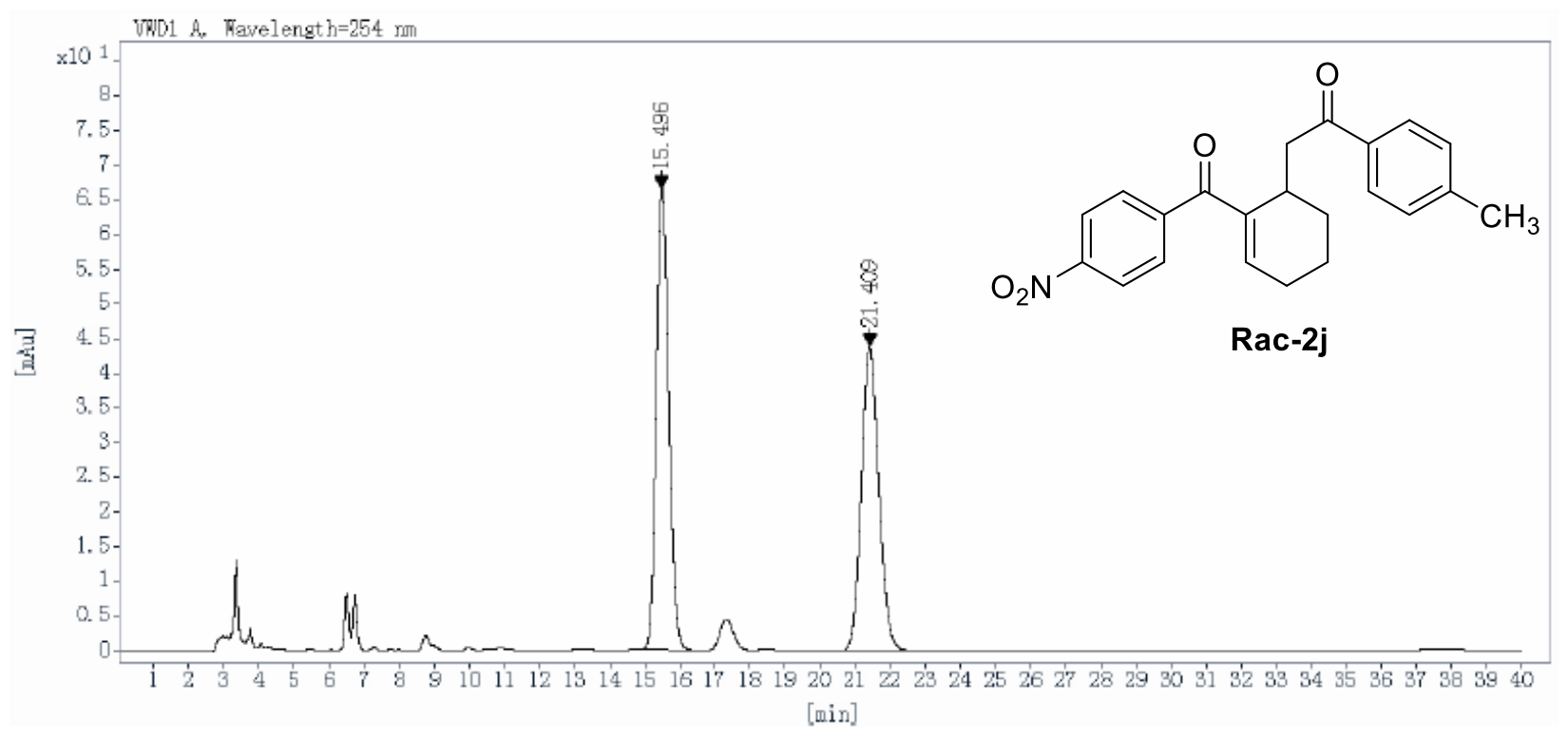

$\begin{array}{cccccc}\begin{array}{c}\text { Ret Time } \\ \text { [min] }\end{array} & \begin{array}{c}\text { Peak } \\ \text { Type }\end{array} & \begin{array}{c}\text { Width } \\ \text { [min] }\end{array} & \begin{array}{c}\text { Height } \\ \text { [mAU] }\end{array} & \begin{array}{c}\text { Area } \\ {\left[\mathbf{m A U}^{*} \text { s] }\right.}\end{array} & \begin{array}{c}\text { Area } \\ {[\%]}\end{array} \\ 15.496 & \text { BB } & 0.38 & 66.4063 & 1626.6851 & 51.7977 \\ 21.409 & \text { BB } & 0.54 & 43.7239 & 1513.7719 & 48.2023 \\ & & & \text { Totals: } & 3140.4569 & 100.0000\end{array}$

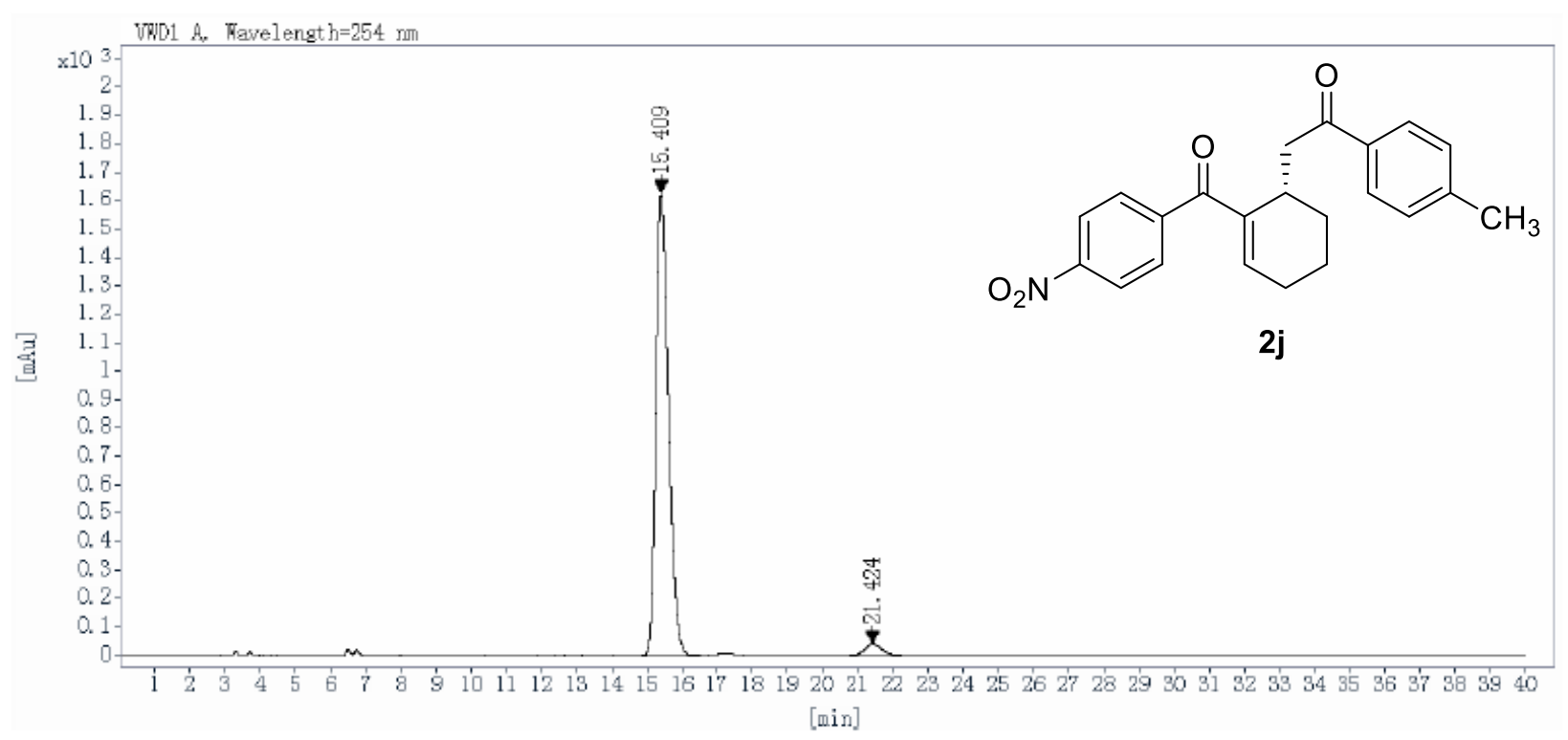

$\begin{array}{cccccc}\begin{array}{c}\text { Ret Time } \\ \text { [min] }\end{array} & \begin{array}{c}\text { Peak } \\ \text { Type }\end{array} & \begin{array}{c}\text { Width } \\ \text { [min] }\end{array} & \begin{array}{c}\text { Height } \\ \text { [mAU] }\end{array} & \begin{array}{c}\text { Area } \\ {\left[\mathbf{m A U}^{*} \text { s] }\right.}\end{array} & \begin{array}{c}\text { Area } \\ {[\%]}\end{array} \\ 15.409 & \text { BB } & 0.38 & 1625.7715 & 40382.8750 & 96.6043 \\ 21.424 & \text { BB } & 0.54 & 40.6351 & 1419.4937 & 3.3957 \\ & & & \text { Totals: } & 41802.3687 & 100.0000\end{array}$


<smiles>CC(=O)C[C@H]1CCCC=C1C(=O)c1ccccc1</smiles>

2k

${ }^{1} \mathrm{H}$ NMR $\left(400 \mathrm{MHz}, \mathrm{CDCl}_{3}\right)$

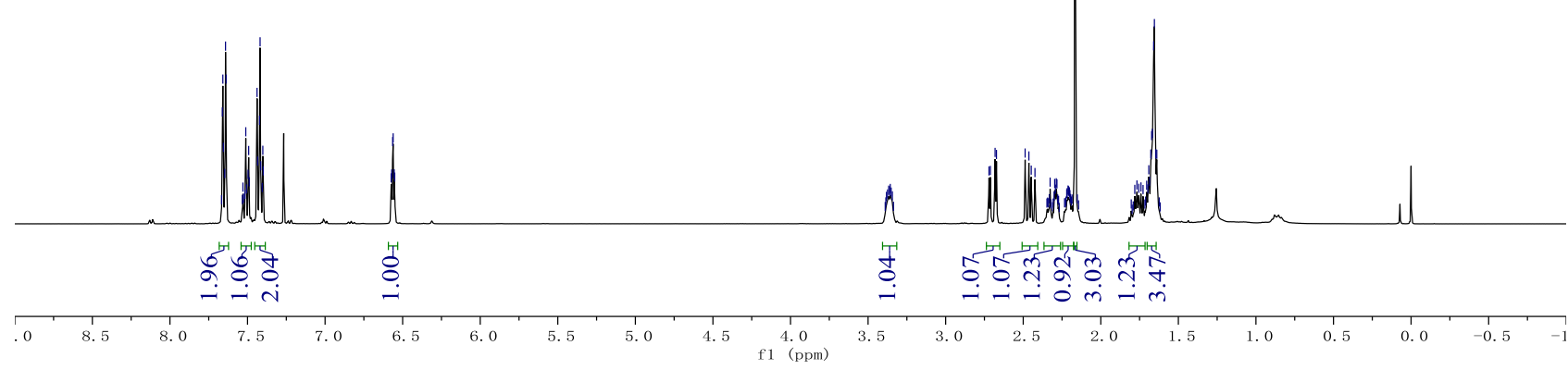

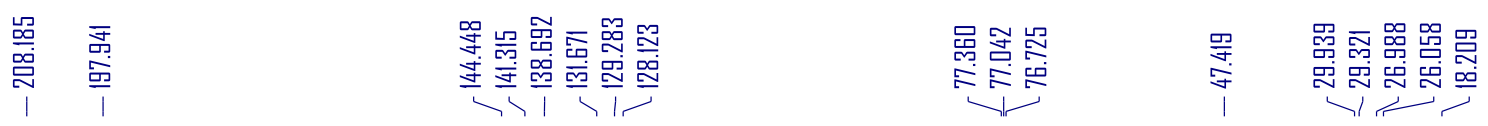<smiles>CC(=O)C[C@H]1CCCC=C1C(=O)c1ccccc1</smiles>

2k

${ }^{13} \mathrm{C}$ NMR $\left(100 \mathrm{MHz}, \mathrm{CDCl}_{3}\right)$
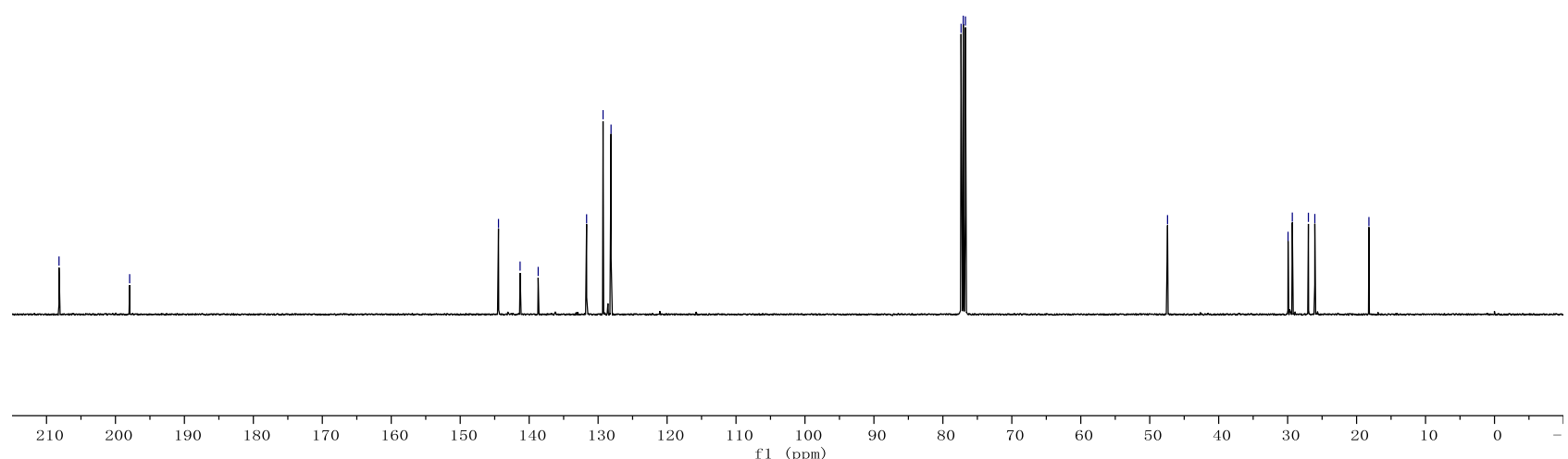
<smiles>CC(=O)C[C@H]1CCCC=C1C(=O)c1ccccc1</smiles>

2k

HRMS (ESI-TOF) m/z: [M + Na] $]^{+}$

Calcd. for $\mathrm{C}_{16} \mathrm{H}_{18} \mathrm{O}_{2} \mathrm{Na} 265.1199$
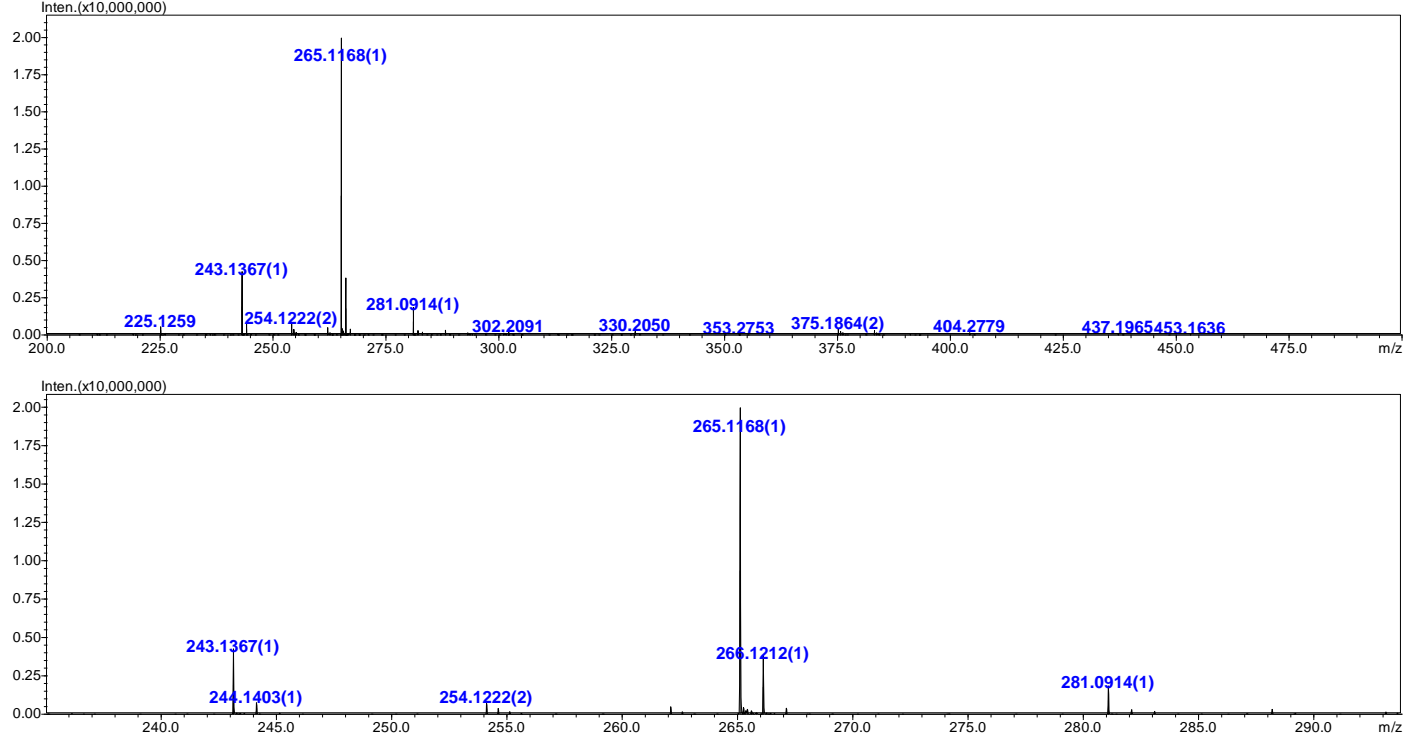


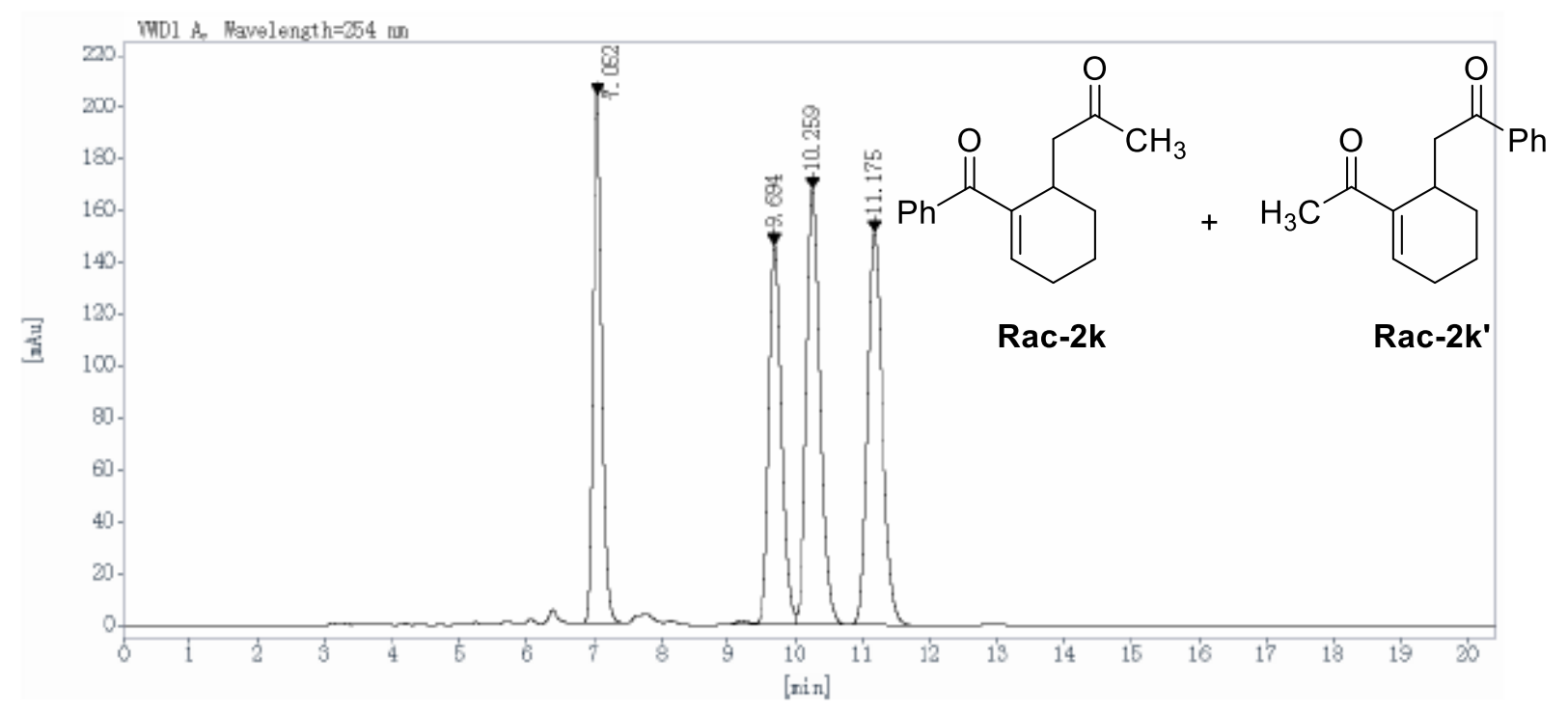

$\begin{array}{cccccc}\begin{array}{c}\text { Ret Time } \\ \text { [min] }\end{array} & \begin{array}{c}\text { Peak } \\ \text { Type }\end{array} & \begin{array}{c}\text { Width } \\ {[\mathrm{min}]}\end{array} & \begin{array}{c}\text { Height } \\ {[\mathrm{mAU}]}\end{array} & \begin{array}{c}\text { Area } \\ {[\mathrm{mAU} * \text { s] }}\end{array} & \begin{array}{c}\text { Area } \\ {[\%]}\end{array} \\ 7.052 & \text { VB R } & 0.14 & 203.8301 & 1910.2672 & 22.2618 \\ 9.694 & \text { VV R } & 0.20 & 146.1806 & 1917.6829 & 22.3482 \\ 10.259 & \text { VB } & 0.22 & 168.0620 & 2375.8555 & 27.6876 \\ 11.175 & \text { BB } & 0.24 & 151.4323 & 2377.1282 & 27.7024 \\ & & & \text { Totals: } & \$ 580.9337 & 100.0000\end{array}$

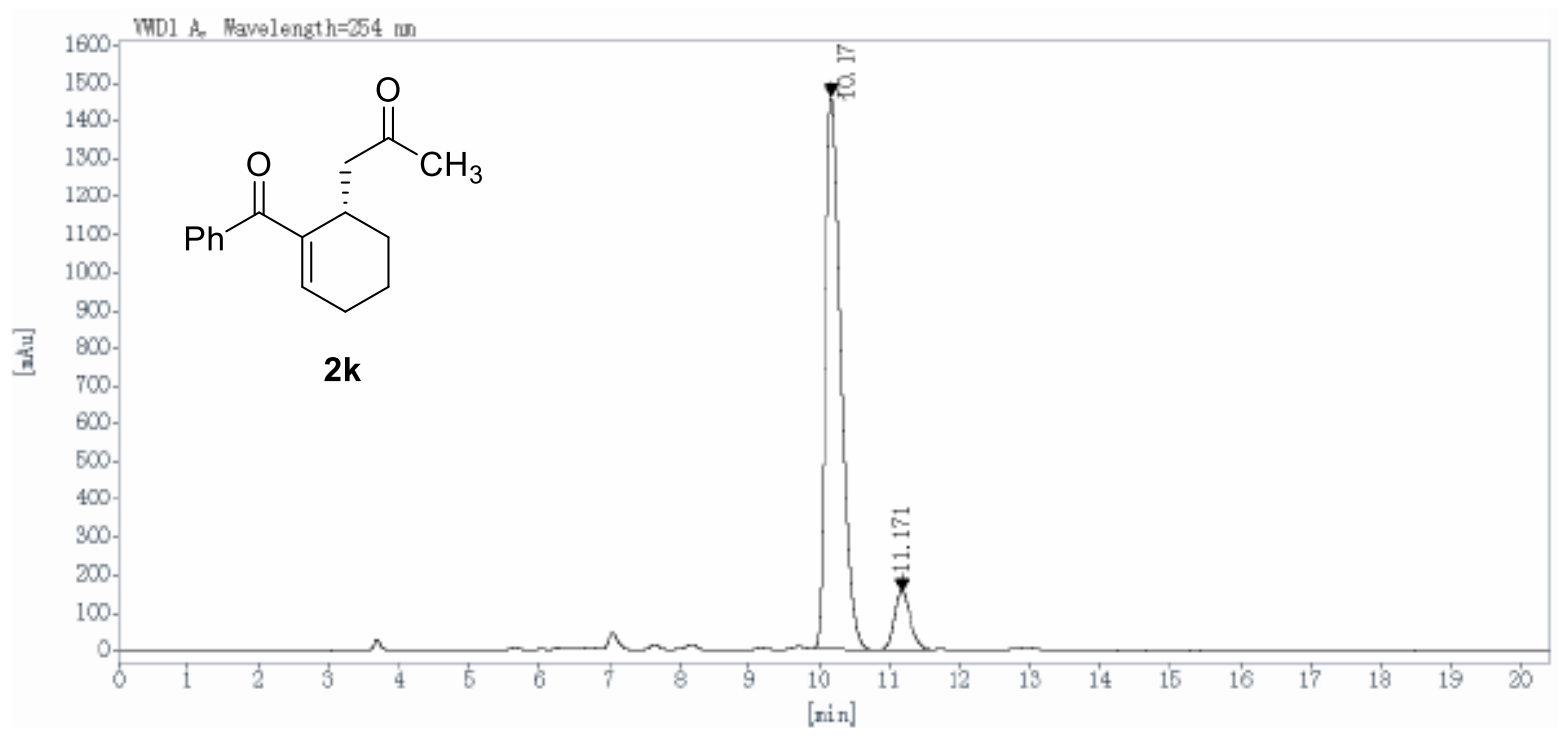

\begin{tabular}{cccccc}
$\begin{array}{c}\text { Ret Time } \\
\text { [min] }\end{array}$ & $\begin{array}{c}\text { Peak } \\
\text { Type }\end{array}$ & $\begin{array}{c}\text { Width } \\
\text { [min] }\end{array}$ & $\begin{array}{c}\text { Height } \\
{[\mathrm{mAU}]}\end{array}$ & $\begin{array}{c}\text { Area } \\
{[\mathrm{mAU} \text { *s] }}\end{array}$ & $\begin{array}{c}\text { Area } \\
{[\%]}\end{array}$ \\
\hline 10.170 & BB & 0.24 & 1464.5920 & 22683.4648 & 90.2456 \\
11.171 & BB & 0.24 & $\begin{array}{c}157.1344 \\
\text { Totals: }\end{array}$ & 2451.7878 & 9.7544 \\
& & & 25135.2527 & 100.0000
\end{tabular}


<smiles>O=C(CC1CCC=C1C(=O)c1ccccc1)c1ccccc1</smiles>

4a

${ }^{1} \mathrm{H}$ NMR $\left(400 \mathrm{MHz}, \mathrm{CDCl}_{3}\right)$

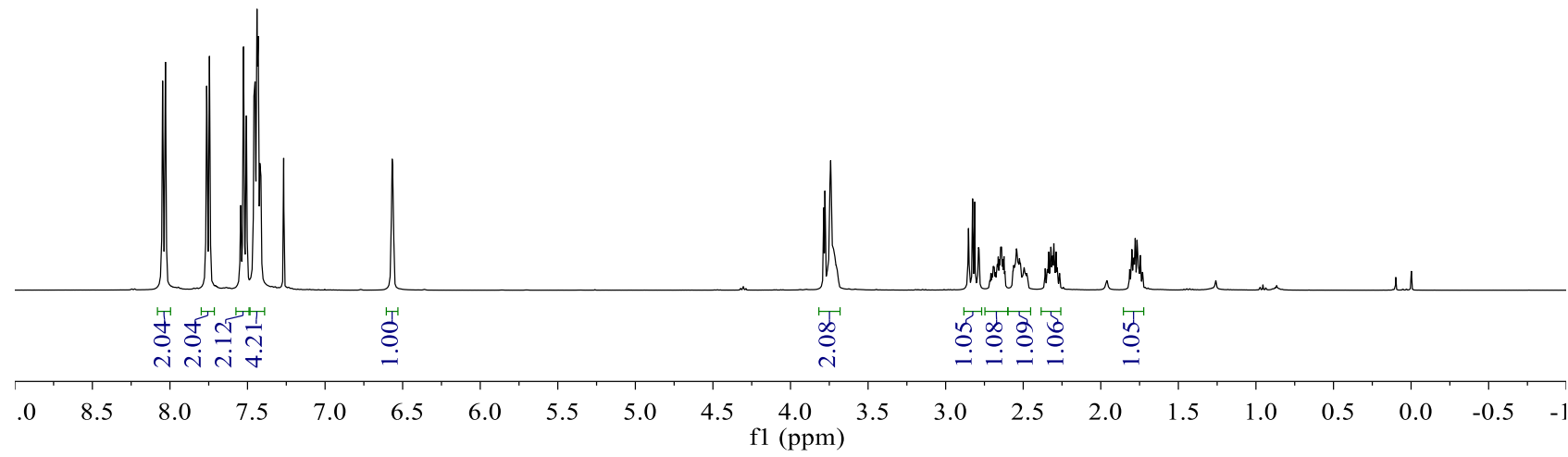

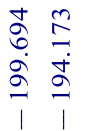

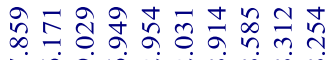

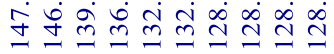

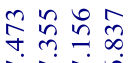

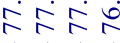

孛全

वंध<smiles>O=C(CC1CCC=C1C(=O)c1ccccc1)c1ccccc1</smiles>

$4 a$

${ }^{13} \mathrm{C}$ NMR $\left(100 \mathrm{MHz}, \mathrm{CDCl}_{3}\right)$

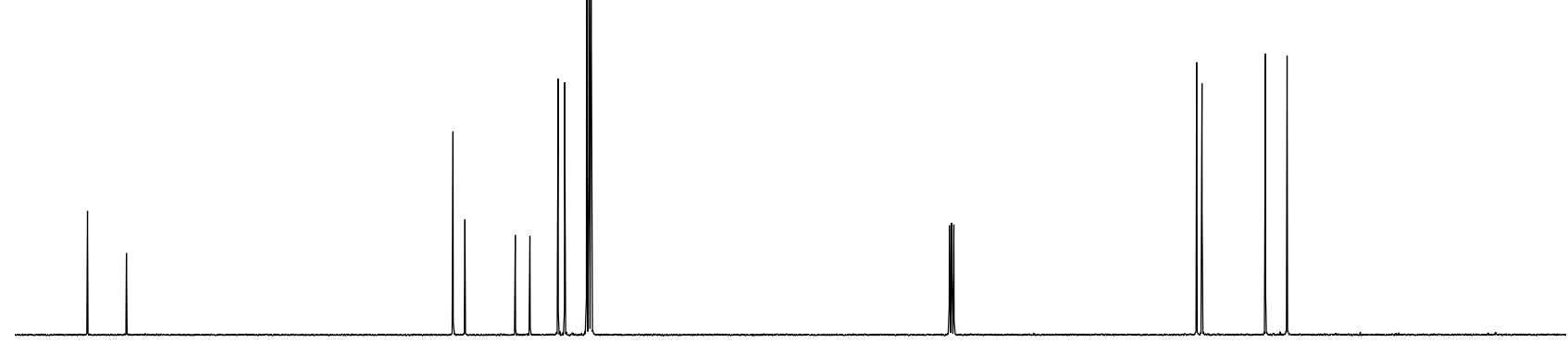

$\begin{array}{lllllllllllllllllllllllll}10 & 200 & 190 & 180 & 170 & 160 & 150 & 140 & 130 & 120 & 110 & \begin{array}{c}100 \\ \mathrm{f} 1(\mathrm{ppm})\end{array} & 90 & 80 & 70 & 60 & 50 & 40 & 30 & 20 & 10 & 0 & -\end{array}$ 


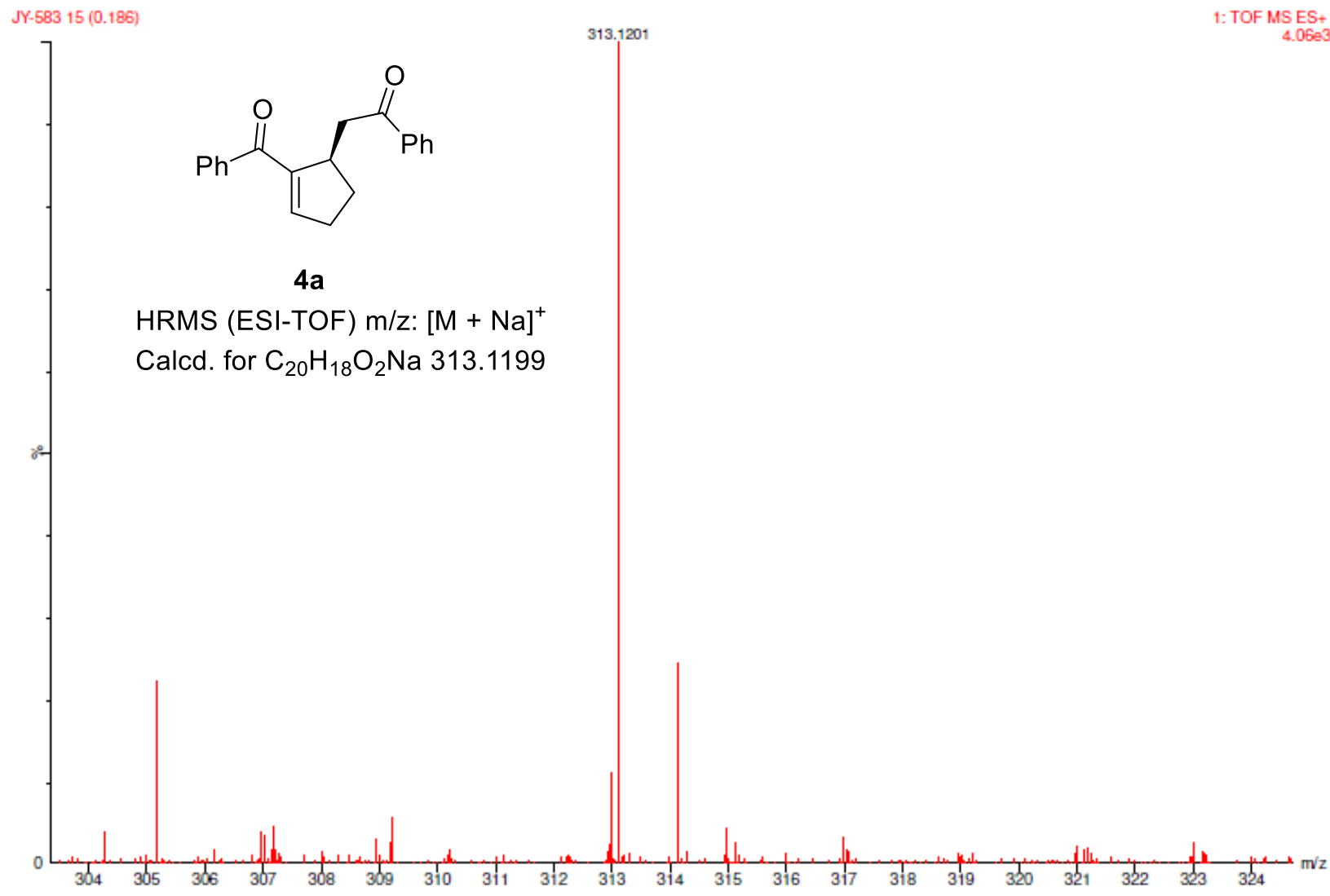




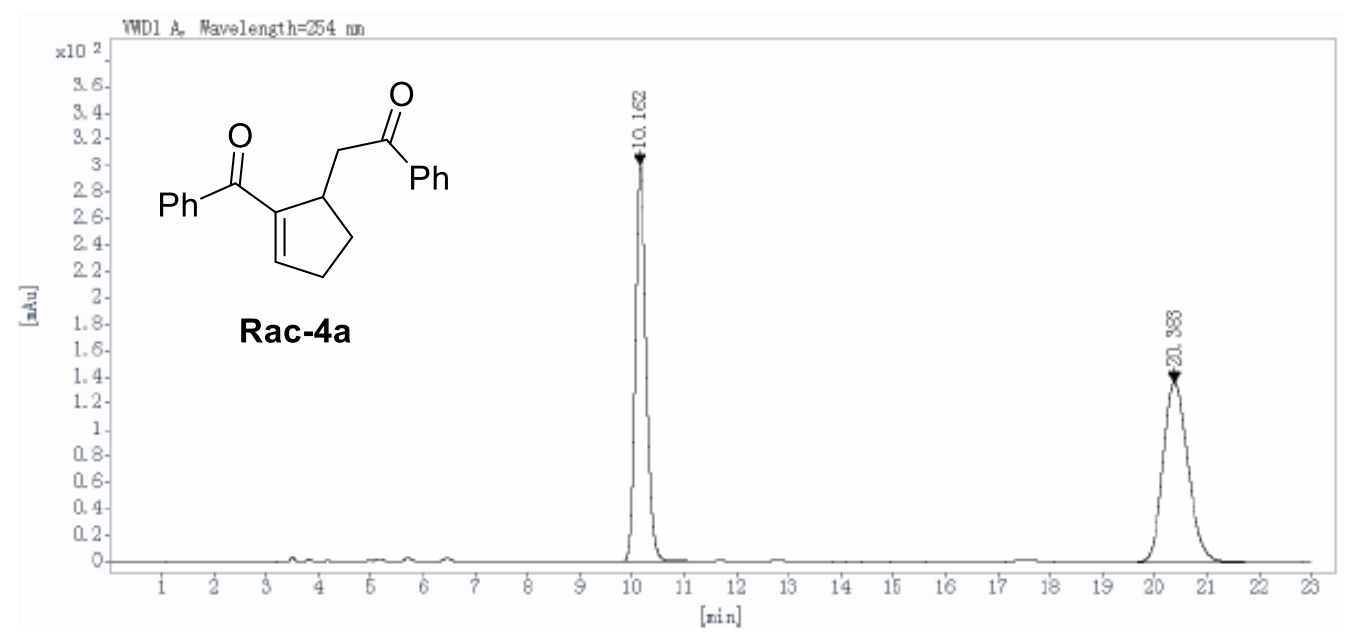

\begin{tabular}{|c|c|c|c|c|c|}
\hline $\begin{array}{l}\text { Ret Time } \\
\text { [min] }\end{array}$ & $\begin{array}{l}\text { Peak } \\
\text { Type }\end{array}$ & $\begin{array}{l}\text { Width } \\
\text { [min] }\end{array}$ & $\begin{array}{l}\text { Height } \\
\text { [mAU] }\end{array}$ & $\underset{\left[\mathrm{mAU}^{*} *_{s}\right]}{\text { Area }}$ & $\begin{array}{l}\text { Area } \\
{[\%]}\end{array}$ \\
\hline 10.162 & BV R & 0.23 & 300.3559 & 4493.3936 & 50.0782 \\
\hline \multirow[t]{2}{*}{20.383} & BB & 0.51 & 136.3989 & 4479.3589 & 49.9218 \\
\hline & & & Totals: & 8972.7524 & 100.0000 \\
\hline
\end{tabular}

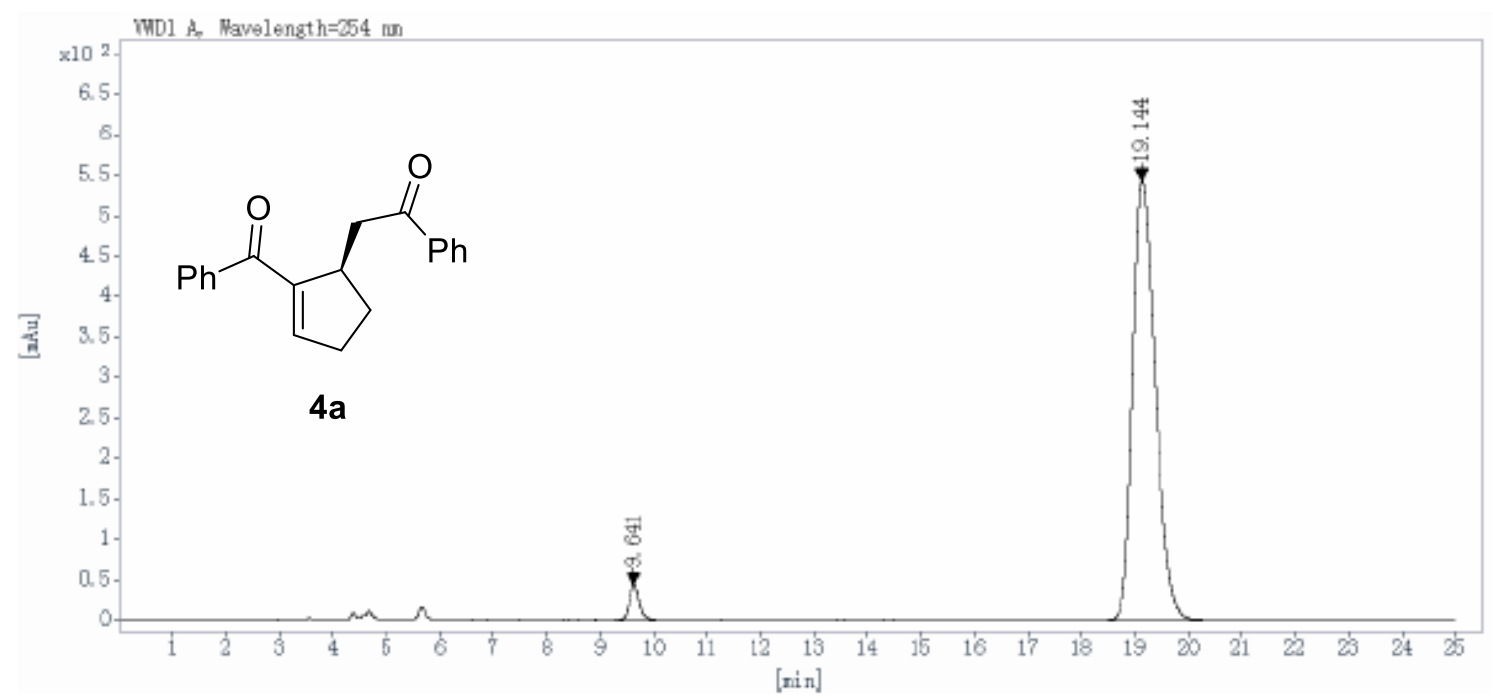

$\begin{array}{cccccc}\begin{array}{c}\text { Ret Time } \\ \text { [min] }\end{array} & \begin{array}{c}\text { Peak } \\ \text { Type }\end{array} & \begin{array}{c}\text { Width } \\ \text { [min] }\end{array} & \begin{array}{c}\text { Height } \\ \text { [mAU] }\end{array} & \begin{array}{c}\text { Area } \\ \text { [mAU*s] }\end{array} & \begin{array}{c}\text { Area } \\ {[\%]}\end{array} \\ 9.641 & \text { BB } & 0.20 & 44.1915 & 581.3834 & 3.5255 \\ 19.144 & \text { BB } & 0.45 & 543.1214 & 15909.2666 & 96.4745 \\ & & & \text { Totals: } & 16490.6500 & 100.0000\end{array}$


<smiles>Cc1ccc(C(=O)CC2CCC=C2C(=O)c2ccc(C)cc2)cc1</smiles>

4b

${ }^{1} \mathrm{H}$ NMR $\left(400 \mathrm{MHz}, \mathrm{CDCl}_{3}\right)$

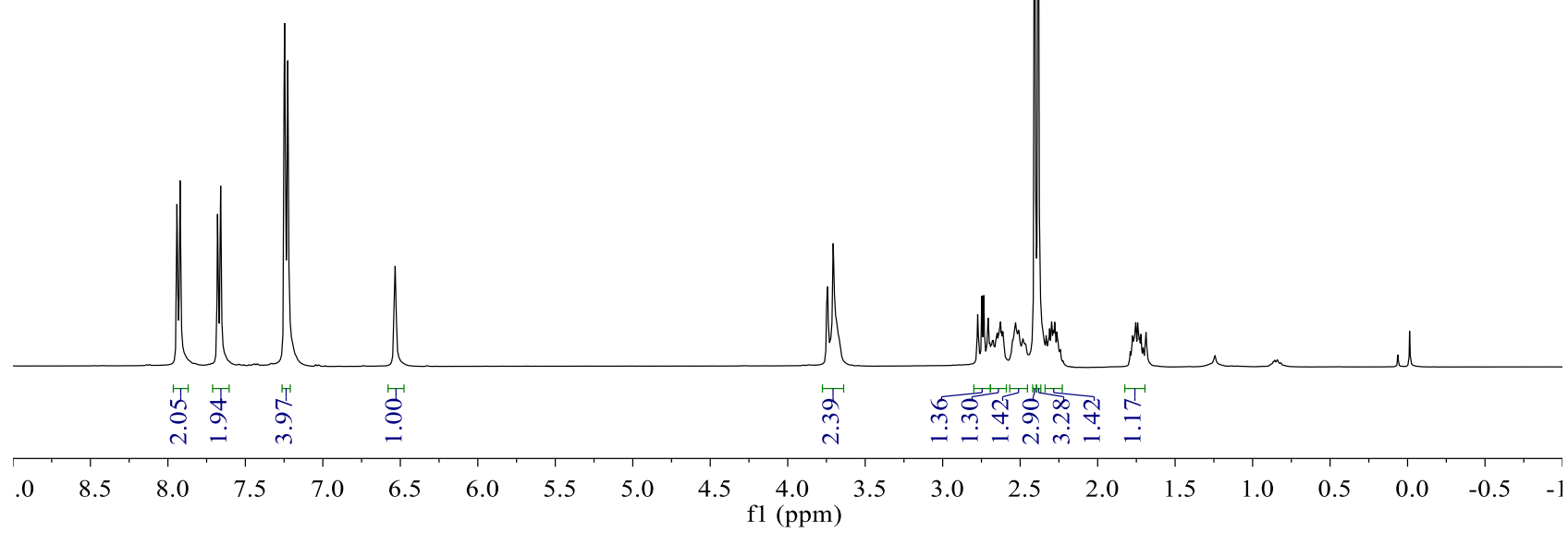

กู่

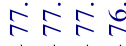

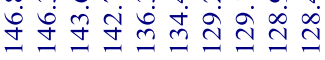

बे<smiles>Cc1ccc(C(=O)C[C@H]2CCC=C2C(=O)c2ccc(C)cc2)cc1</smiles>

4b

${ }^{13} \mathrm{C} \mathrm{NMR}\left(100 \mathrm{MHz}, \mathrm{CDCl}_{3}\right)$

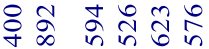

รี तึจิ तี

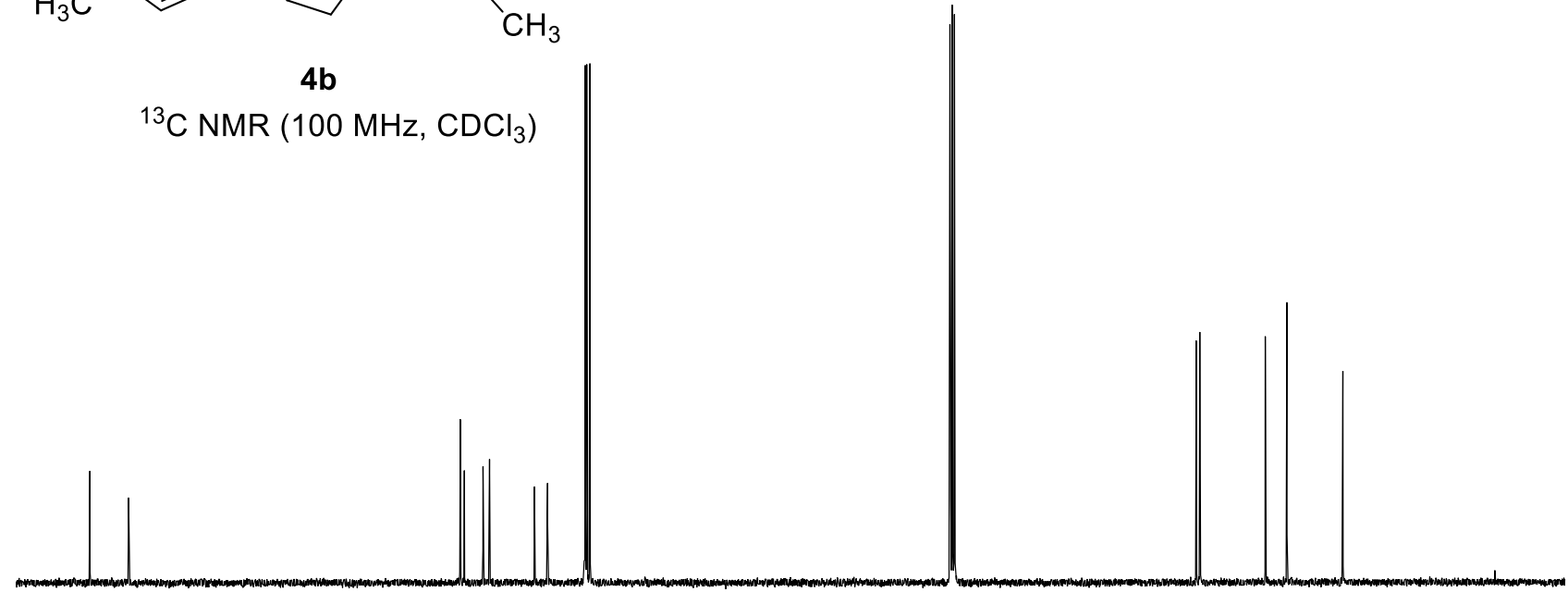

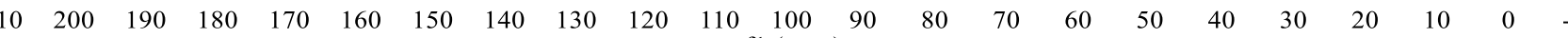




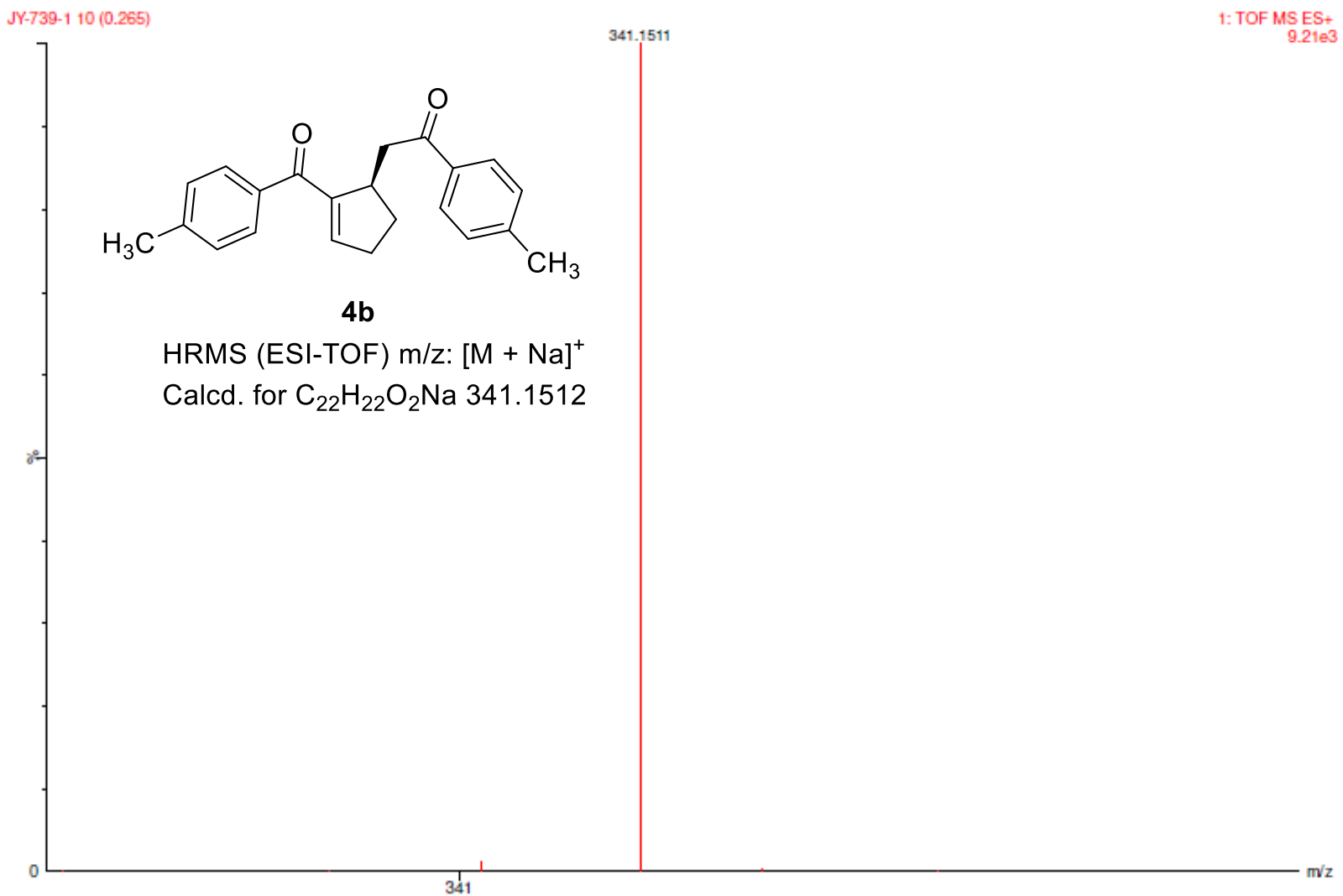




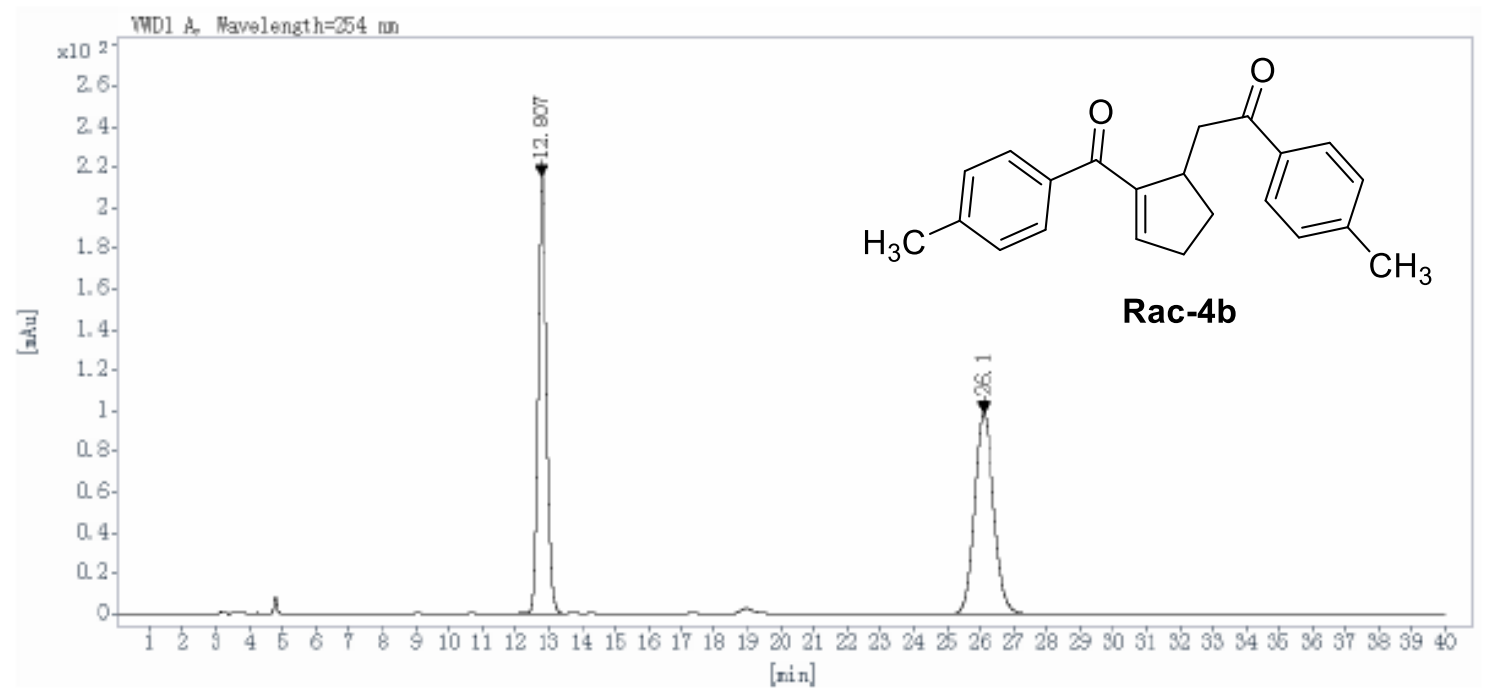

\begin{tabular}{cccccc}
$\begin{array}{c}\text { Ret Time } \\
\text { [min] }\end{array}$ & $\begin{array}{c}\text { Peak } \\
\text { Type }\end{array}$ & $\begin{array}{c}\text { Width } \\
{[\mathrm{min}]}\end{array}$ & $\begin{array}{c}\text { Height } \\
{[\mathrm{mAU}]}\end{array}$ & $\begin{array}{c}\text { Area } \\
{\left[\mathrm{mAU} *^{*}\right]}\end{array}$ & $\begin{array}{c}\text { Area } \\
{[\%]}\end{array}$ \\
\hline 12.807 & VB R & 0.29 & 214.7789 & 3987.1458 & 50.0071 \\
26.100 & BB & 0.62 & 99.0458 & 3986.0110 & 49.9929 \\
& & & Totals: & 7973.1567 & 100.0000
\end{tabular}

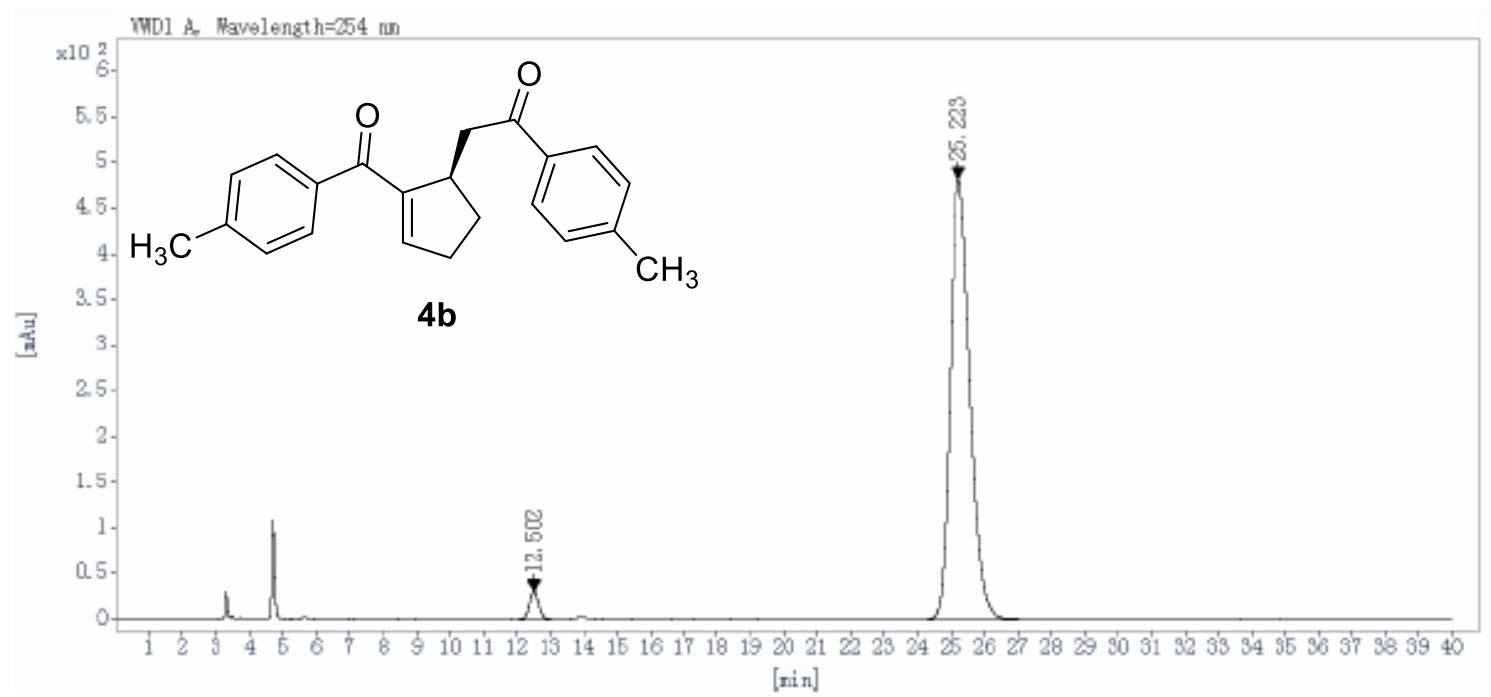

\begin{tabular}{|c|c|c|c|c|c|}
\hline $\begin{array}{l}\text { Ret Time } \\
\text { [min] }\end{array}$ & $\begin{array}{l}\text { Peak } \\
\text { Type }\end{array}$ & $\begin{array}{l}\text { Width } \\
\text { [min] }\end{array}$ & $\begin{array}{l}\text { Height } \\
\text { [mAU] }\end{array}$ & $\underset{\left[\mathrm{mAU}^{*}{ }^{\mathrm{s}}\right]}{\text { Area }}$ & $\begin{array}{l}\text { Area } \\
{[\%]}\end{array}$ \\
\hline 12.502 & BBA & 0.28 & 31.1235 & 563.8909 & 2.8053 \\
\hline 25.223 & BB & 0.63 & 481.4982 & 19536.7715 & 97.1947 \\
\hline
\end{tabular}


<smiles>O=C(C[C@@H]1CCC=C1C(=O)c1ccc(Br)cc1)c1ccc(Br)cc1</smiles>

$4 \mathrm{c}$

${ }^{1} \mathrm{H}$ NMR $\left(400 \mathrm{MHz}, \mathrm{CDCl}_{3}\right)$

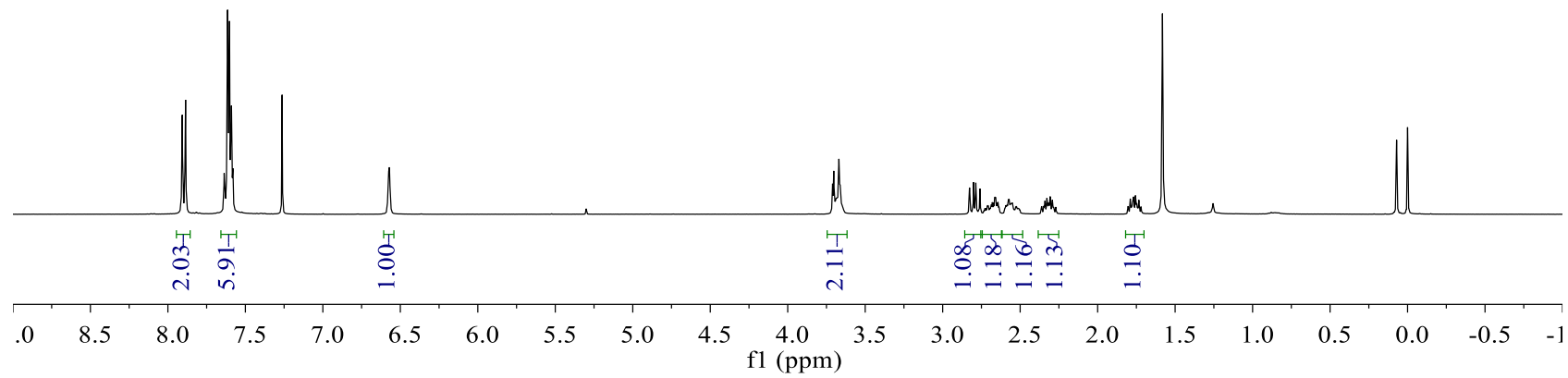

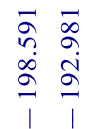

๙ิ)

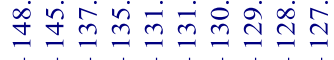

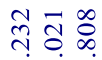

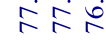

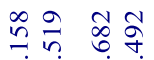

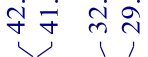<smiles>O=C(CC1CCC=C1C(=O)c1ccc(Br)cc1)c1ccc(Br)cc1</smiles>

$4 c$

${ }^{13} \mathrm{C}$ NMR $\left(150 \mathrm{MHz}, \mathrm{CDCl}_{3}\right)$

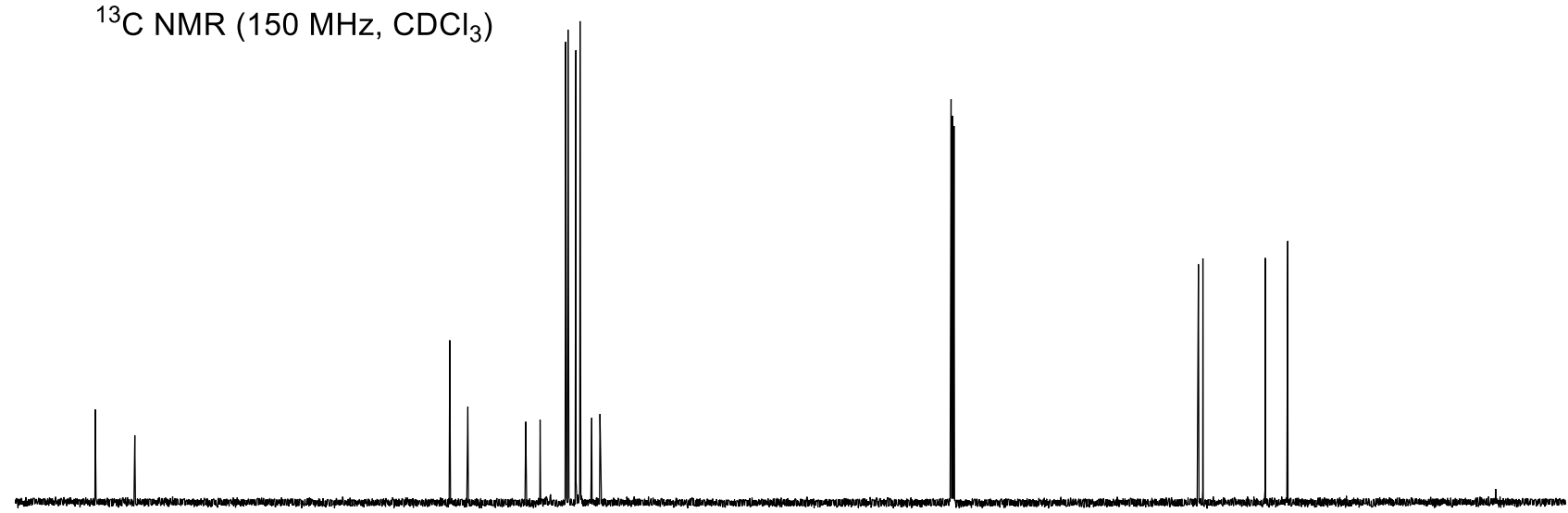

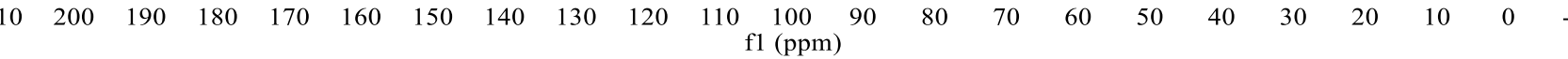



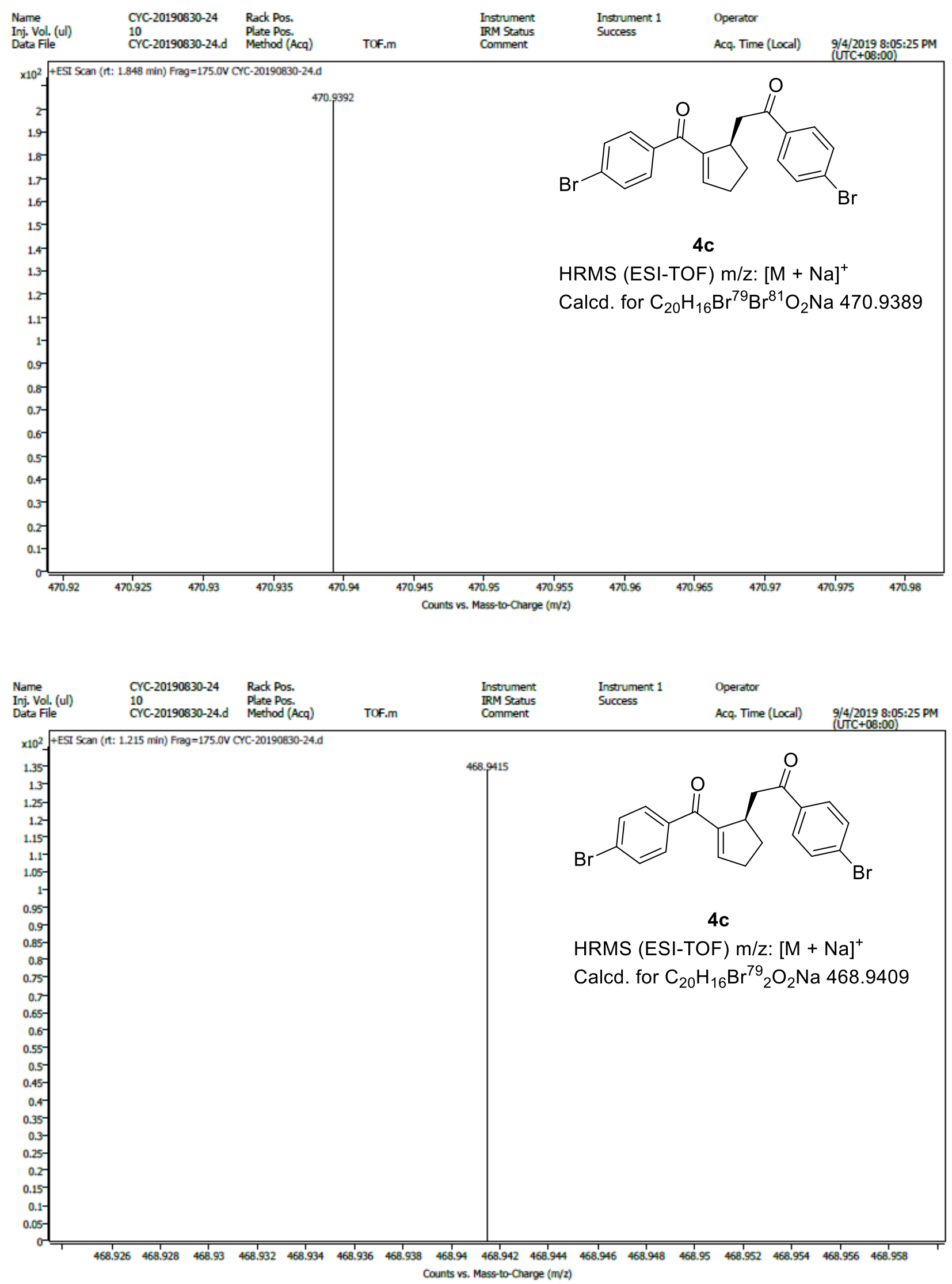


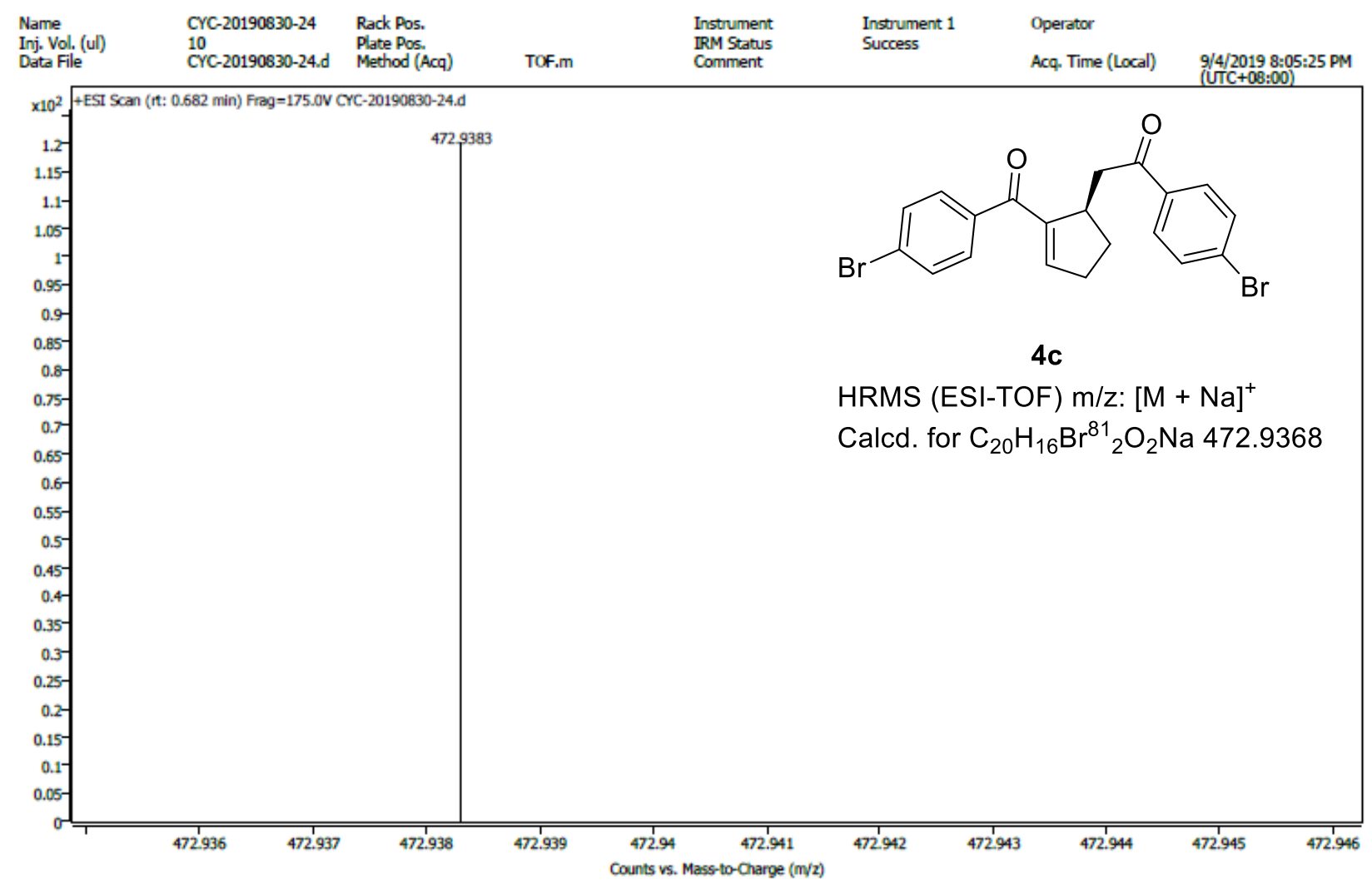




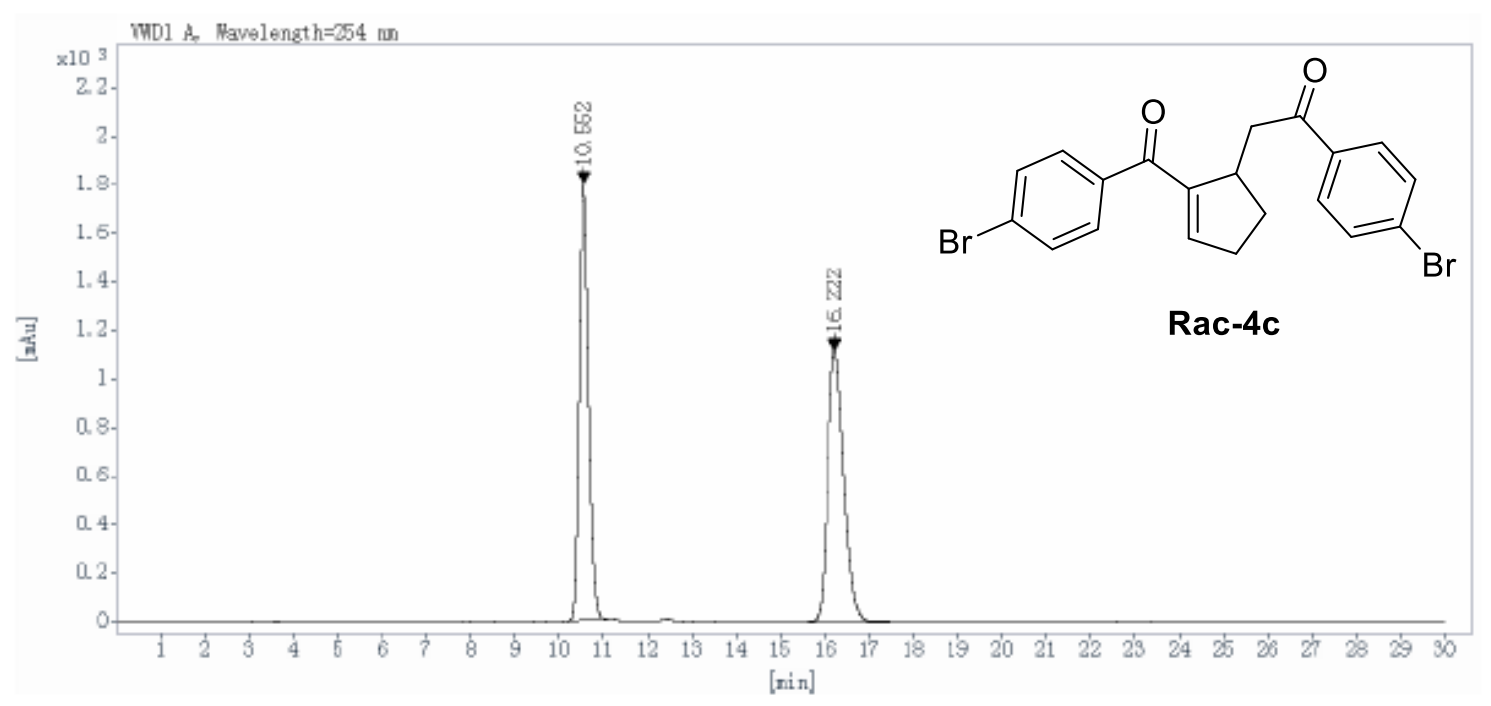

\begin{tabular}{cccccc}
$\begin{array}{c}\text { Ret Time } \\
\text { [min] }\end{array}$ & $\begin{array}{c}\text { Peak } \\
\text { Type }\end{array}$ & $\begin{array}{c}\text { Width } \\
{[\mathrm{min}]}\end{array}$ & $\begin{array}{c}\text { Height } \\
{[\mathrm{mAU}]}\end{array}$ & $\begin{array}{c}\text { Area } \\
{\left[\mathrm{mAU}^{*} \text { s] }\right.}\end{array}$ & $\begin{array}{c}\text { Area } \\
{[\%]}\end{array}$ \\
\hline 10.552 & BV R & 0.24 & 1796.9254 & 27576.1777 & 49.6206 \\
16.222 & BB & 0.39 & 1118.7966 & 27997.8242 & 50.3794 \\
& & & Totals: & 55574.0020 & 100.0000
\end{tabular}

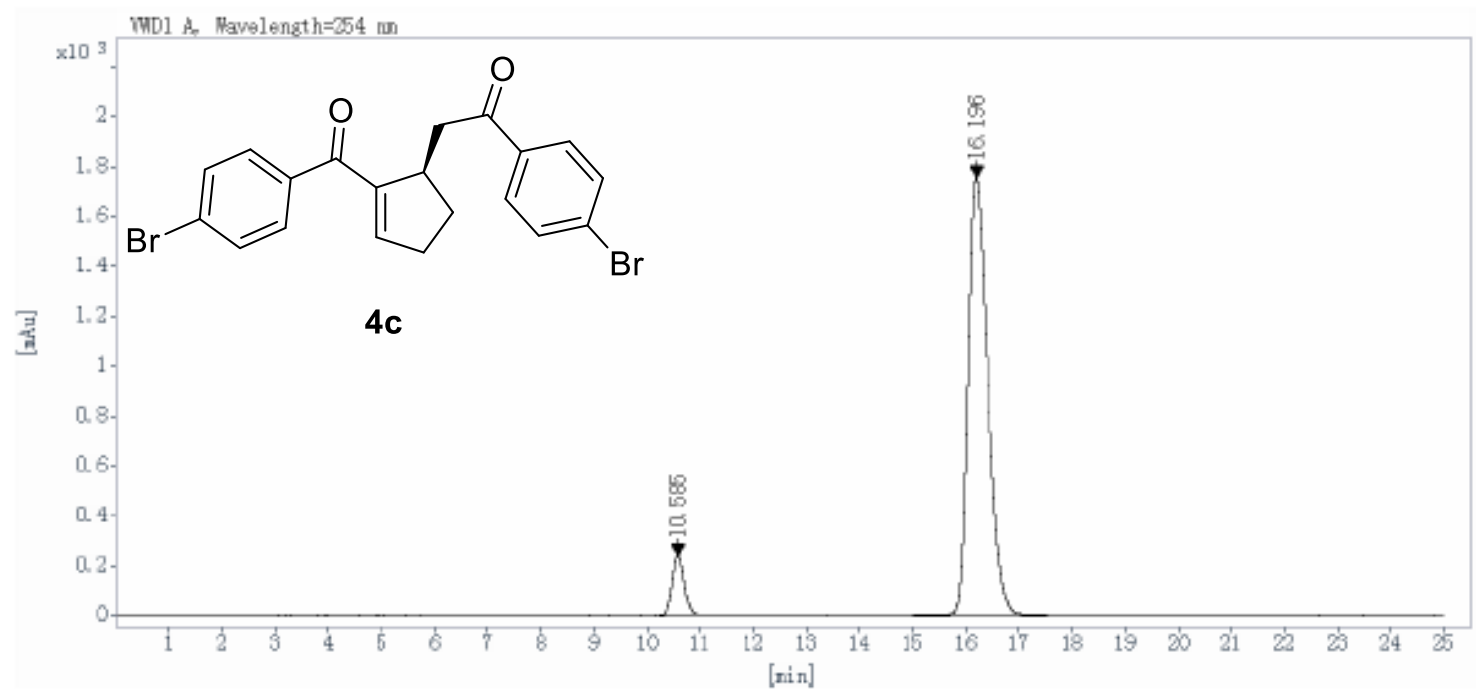

\begin{tabular}{cccccc}
$\begin{array}{c}\text { Ret Time } \\
{[\mathrm{min}]}\end{array}$ & $\begin{array}{c}\text { Peak } \\
\text { Type }\end{array}$ & $\begin{array}{c}\text { Width } \\
{[\mathrm{min}]}\end{array}$ & $\begin{array}{c}\text { Height } \\
{[\mathrm{mAU}]}\end{array}$ & $\begin{array}{c}\text { Area } \\
{\left[\mathrm{mAU} \mathbf{*}^{*}\right]}\end{array}$ & $\begin{array}{c}\text { Area } \\
{[\%]}\end{array}$ \\
\hline 10.585 & BB & 0.23 & 240.4097 & 3645.5303 & 7.5076 \\
16.196 & BB & 0.40 & 1753.1029 & 44912.1406 & 92.4924 \\
& & & Totals: & 48557.6709 & 100.0000
\end{tabular}


<smiles>O=C(CC1CCC=C1C(=O)c1ccc2ccccc2c1)c1ccc2ccccc2c1</smiles>

4d

${ }^{1} \mathrm{H}$ NMR $\left(400 \mathrm{MHz}, \mathrm{CDCl}_{3}\right)$

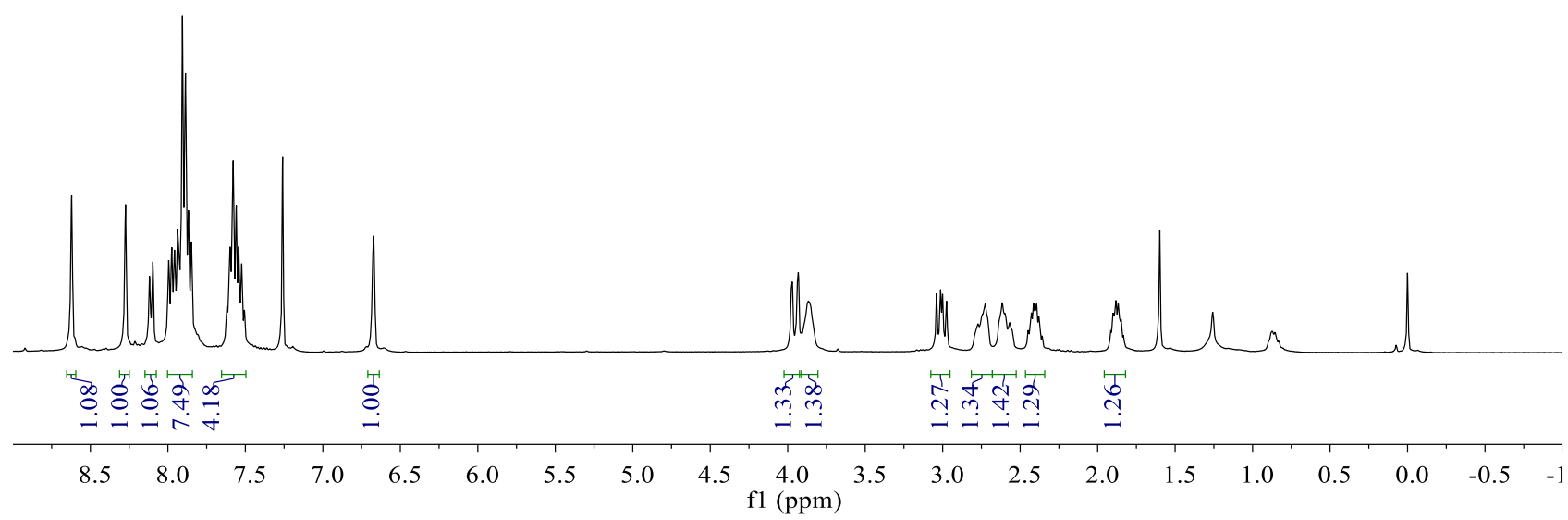

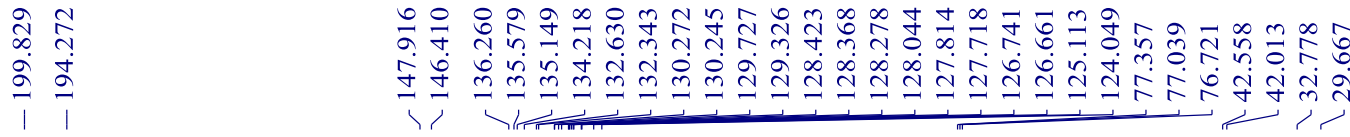<smiles>O=C(CC1CCC=C1C(=O)c1ccc2ccccc2c1)c1ccc2ccccc2c1</smiles>

4d

${ }^{13} \mathrm{C}$ NMR $\left(100 \mathrm{MHz}, \mathrm{CDCl}_{3}\right)$

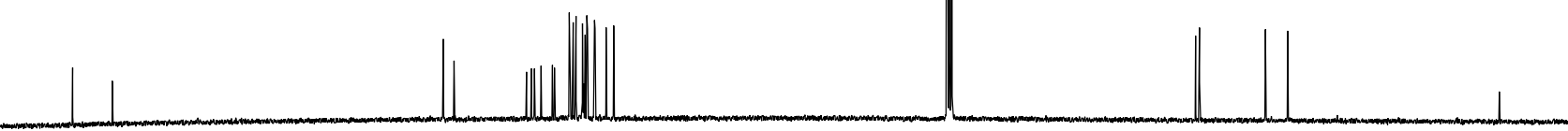

$\begin{array}{llllllllllllllllllllllll}10 & 200 & 190 & 180 & 170 & 160 & 150 & 140 & 130 & 120 & 110 & \begin{array}{c}100 \\ \mathrm{f} 1(\mathrm{ppm})\end{array} & 90 & 80 & 70 & 60 & 50 & 40 & 30 & 20 & 10 & 0 & -\end{array}$ 


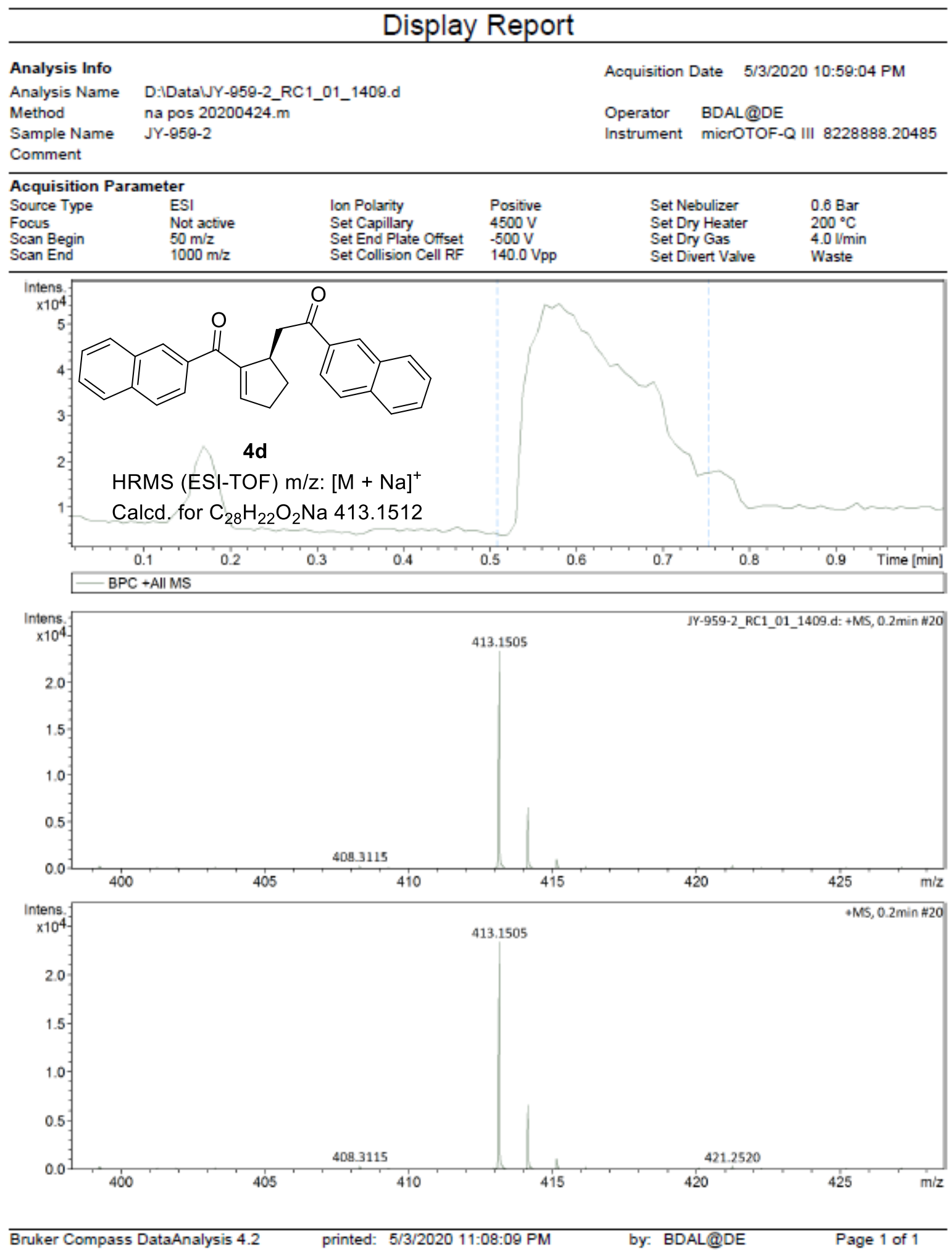




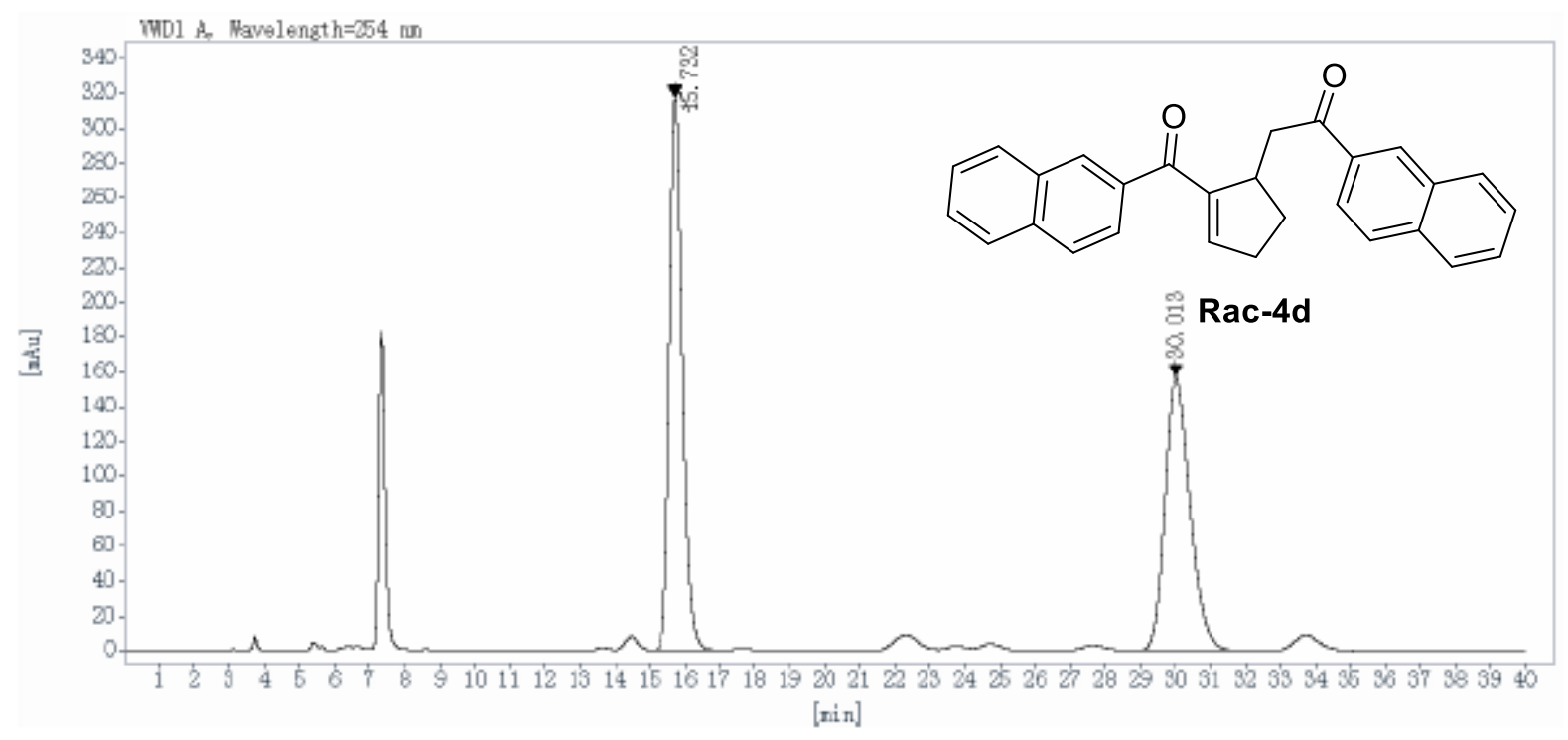

$\begin{array}{cccccc}\begin{array}{c}\text { Ret Time } \\ \text { [min] }\end{array} & \begin{array}{c}\text { Peak } \\ \text { Type }\end{array} & \begin{array}{c}\text { Width } \\ \text { [min] }\end{array} & \begin{array}{c}\text { Height } \\ \text { [mAU] }\end{array} & \begin{array}{c}\text { Area } \\ \text { [mAU*'s] }\end{array} & \begin{array}{c}\text { Area } \\ {[\%]}\end{array} \\ 15.732 & \text { BB } & 0.39 & 317.7285 & 8003.9961 & 50.3952 \\ 30.013 & \text { BB } & 0.77 & \begin{array}{c}157.3561 \\ \text { Totals: }\end{array} & 7878.4487 & 49.6048 \\ & & & & & \end{array}$

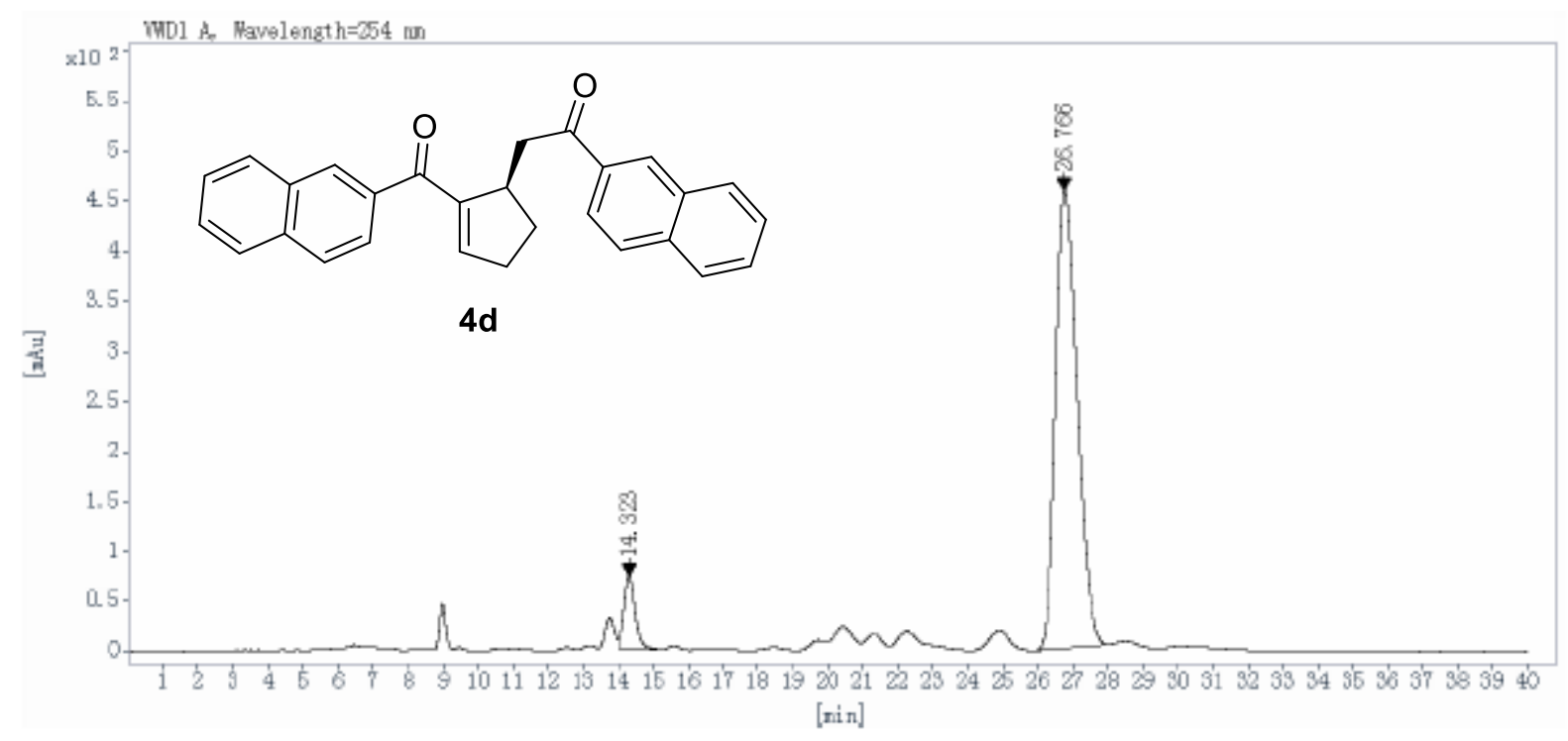

\begin{tabular}{cccccc}
$\begin{array}{c}\text { Ret Time } \\
\text { [min] }\end{array}$ & $\begin{array}{c}\text { Peak } \\
\text { Type }\end{array}$ & $\begin{array}{c}\text { Width } \\
{[\mathrm{min}]}\end{array}$ & $\begin{array}{c}\text { Height } \\
{[\mathrm{mAU}]}\end{array}$ & $\begin{array}{c}\text { Area } \\
{\left[\mathrm{mAU} *^{*} \text { ] }\right.}\end{array}$ & $\begin{array}{c}\text { Area } \\
{[\%]}\end{array}$ \\
\hline 14.323 & VB & 0.34 & 73.7438 & 1642.9812 & 7.7496 \\
26.766 & BB & 0.66 & 457.6746 & 19557.9863 & 92.2504 \\
& & & Totals: & 21200.9675 & 100.0000
\end{tabular}


<smiles>O=C(C[C@H]1CCC=C1C(=O)c1cccs1)c1cccs1</smiles>

$4 e$

${ }^{1} \mathrm{H}$ NMR $\left(400 \mathrm{MHz}, \mathrm{CDCl}_{3}\right)$

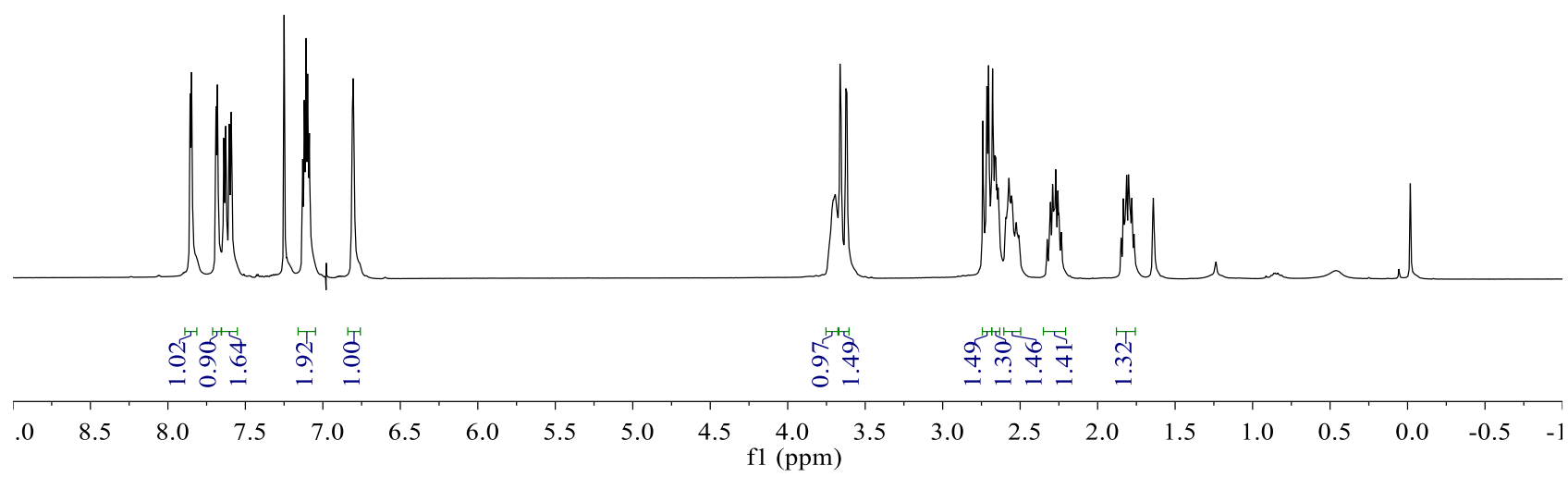<smiles>O=C(C[C@H]1CCC=C1C(=O)c1cccs1)c1cccs1</smiles>

$4 e$

${ }^{13} \mathrm{C}$ NMR $\left(100 \mathrm{MHz}, \mathrm{CDCl}_{3}\right)$

守品

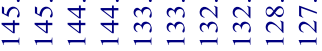

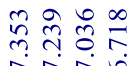

소용

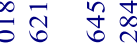

भ भ
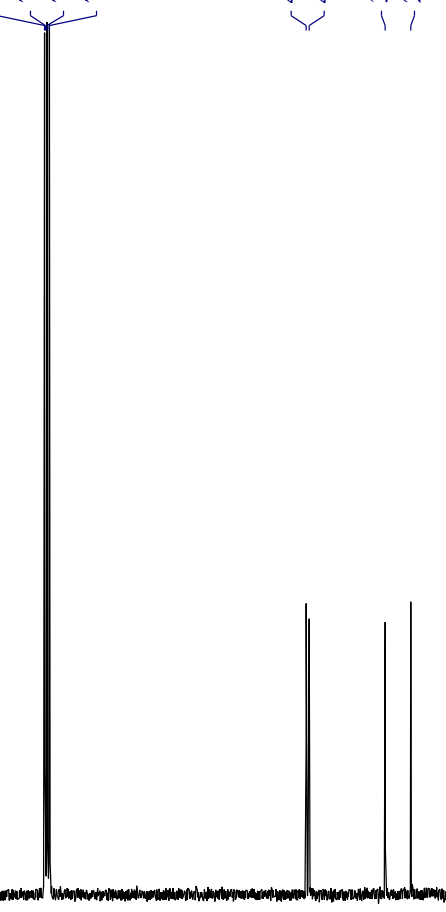

$\begin{array}{lllllllllllllllllllllll}10 & 200 & 190 & 180 & 170 & 160 & 150 & 140 & 130 & 120 & 110 \underset{\mathrm{f} 1(\mathrm{ppm})}{100} & 90 & 80 & 70 & 60 & 50 & 40 & 30 & 20 & 10 & 0 & -\end{array}$ 


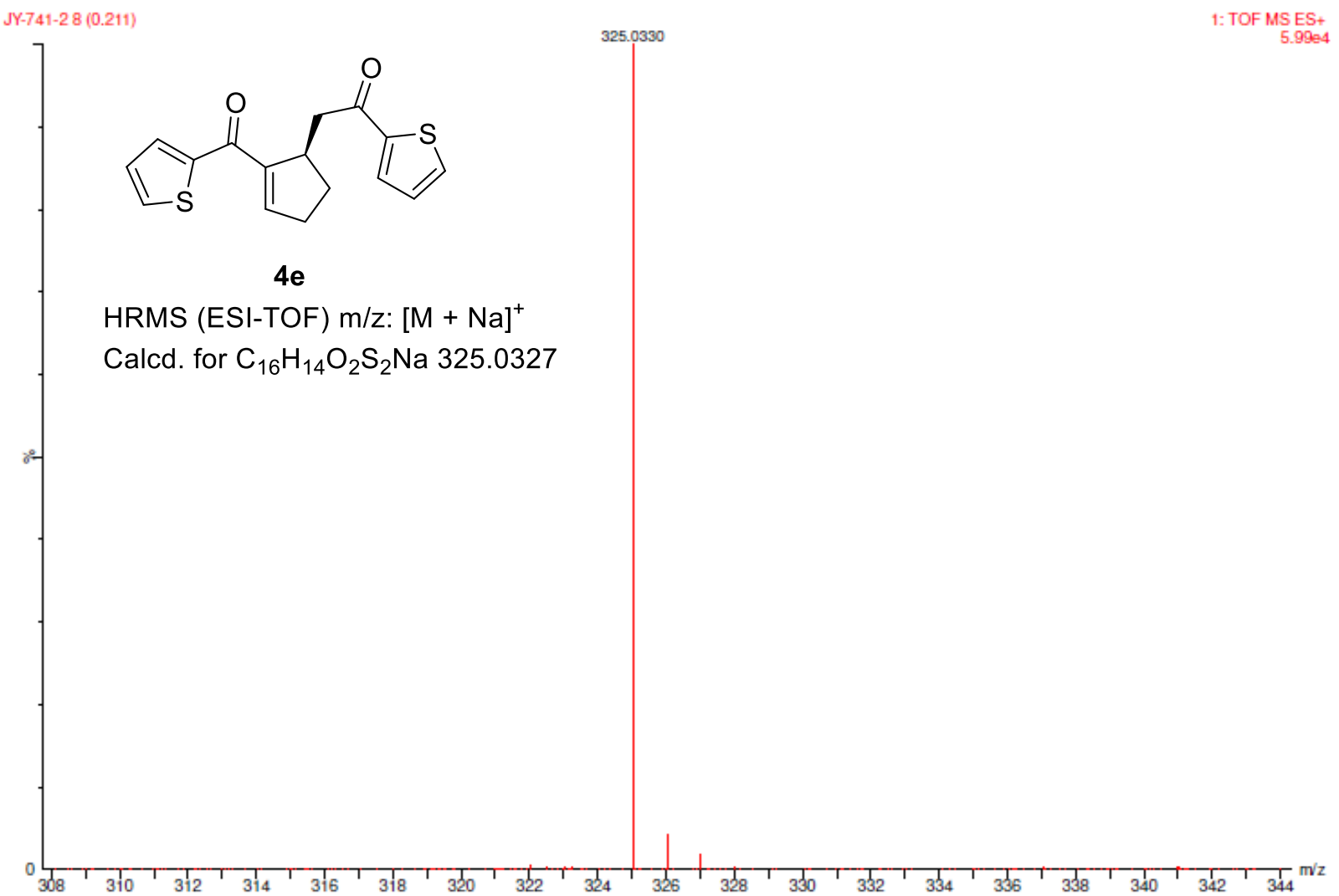




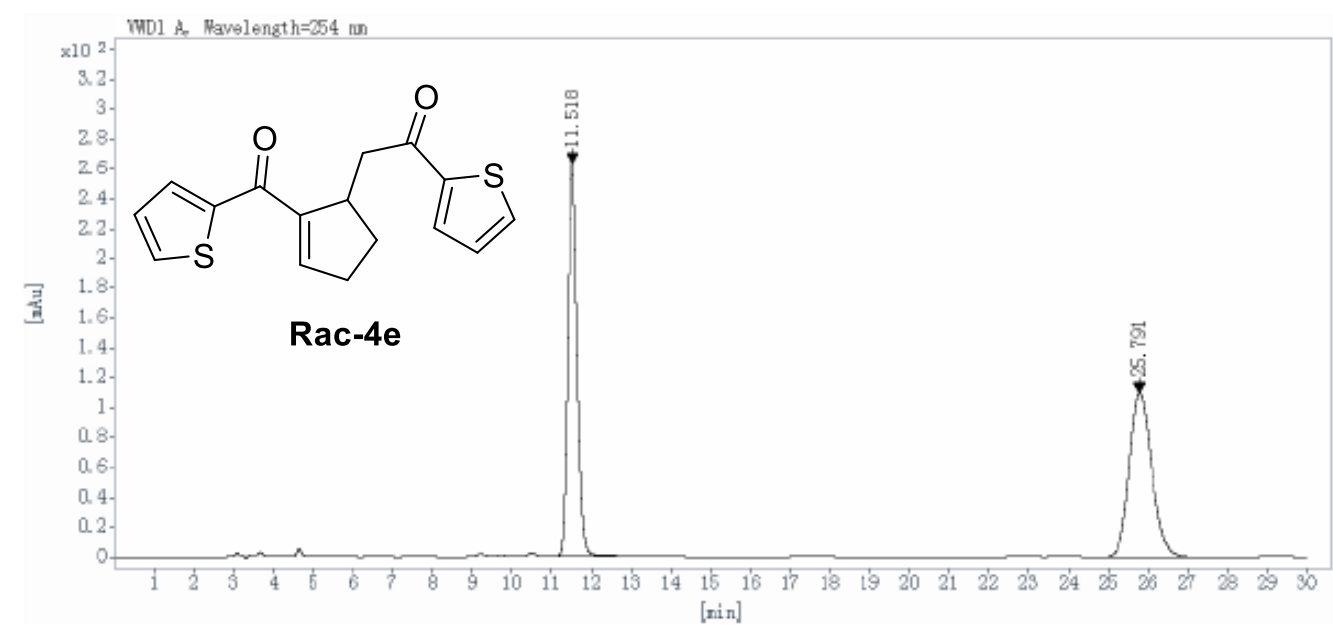

\begin{tabular}{|c|c|c|c|c|c|}
\hline $\begin{array}{l}\text { Ret Time } \\
\text { [min] }\end{array}$ & $\begin{array}{l}\text { Peak } \\
\text { Type }\end{array}$ & $\begin{array}{l}\text { Width } \\
\text { [min] }\end{array}$ & $\begin{array}{l}\text { Height } \\
\text { [mAU] }\end{array}$ & $\begin{array}{c}\text { Area } \\
{\left[\mathrm{mAU}^{*} s\right]}\end{array}$ & $\begin{array}{l}\text { Area } \\
{[\%]}\end{array}$ \\
\hline 11.518 & BV R & 0.26 & 263.0129 & 4377.5229 & 50.2231 \\
\hline 25.791 & BB & 0.61 & 110.0018 & 4338.6396 & 49.7769 \\
\hline & & & Totals: & 8716.1626 & 100.0000 \\
\hline
\end{tabular}

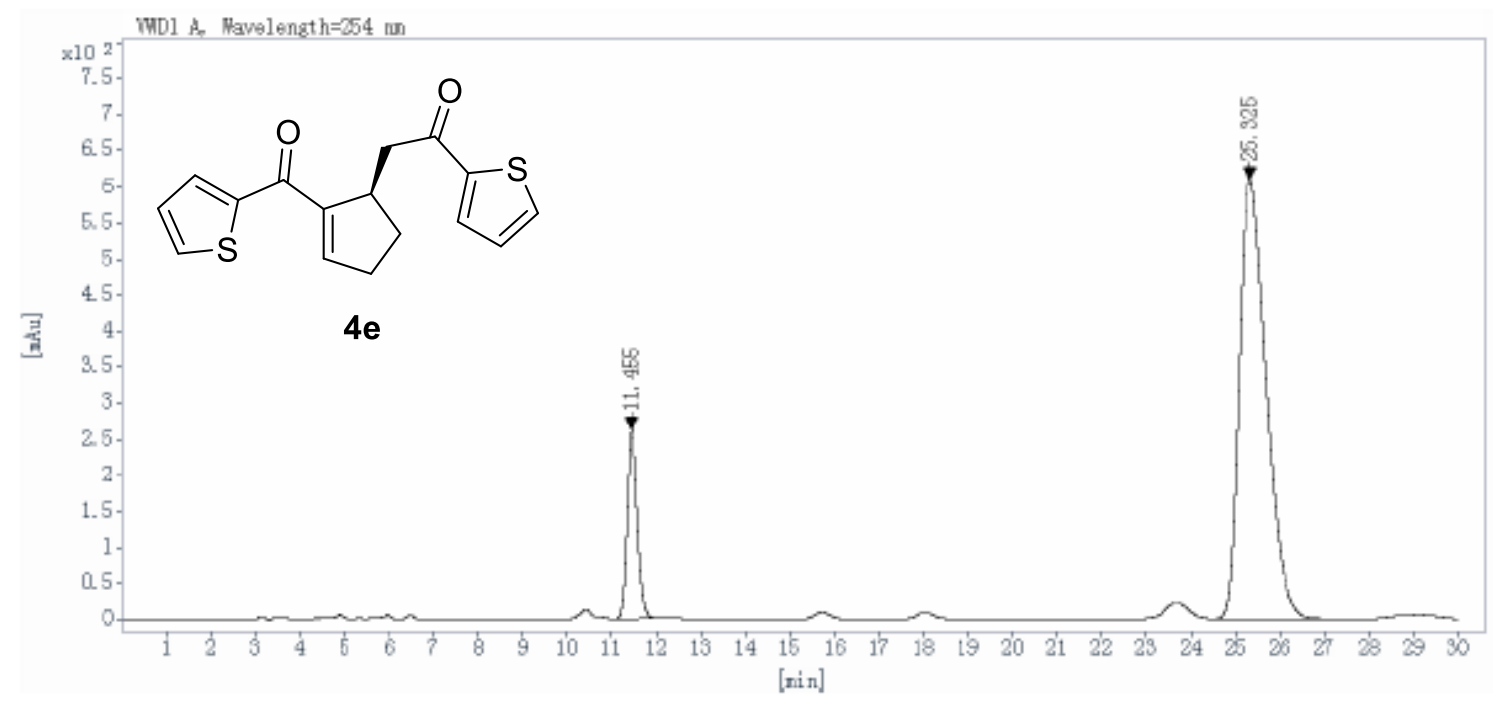

\begin{tabular}{cccccc}
$\begin{array}{c}\text { Ret Time } \\
\text { [min] }\end{array}$ & $\begin{array}{c}\text { Peak } \\
\text { Type }\end{array}$ & $\begin{array}{c}\text { Width } \\
{[\mathrm{min}]}\end{array}$ & $\begin{array}{c}\text { Height } \\
{[\mathrm{mAU}]}\end{array}$ & $\begin{array}{c}\text { Area } \\
{[\mathrm{mAU} * \text { s] }}\end{array}$ & $\begin{array}{c}\text { Area } \\
{[\%]}\end{array}$ \\
\hline 11.455 & BB & 0.25 & 263.5360 & 4292.0288 & 14.3889 \\
25.325 & BB & 0.65 & 609.7360 & 25536.7441 & 85.6111 \\
& & & Totals: & 29828.7729 & 100.0000
\end{tabular}


<smiles>C[C@H]1CC=C(C(=O)c2ccccc2)[C@H](CC(=O)c2ccccc2)C1</smiles>

$6 a$

${ }^{1} \mathrm{H}$ NMR $\left(400 \mathrm{MHz}, \mathrm{CDCl}_{3}\right)$

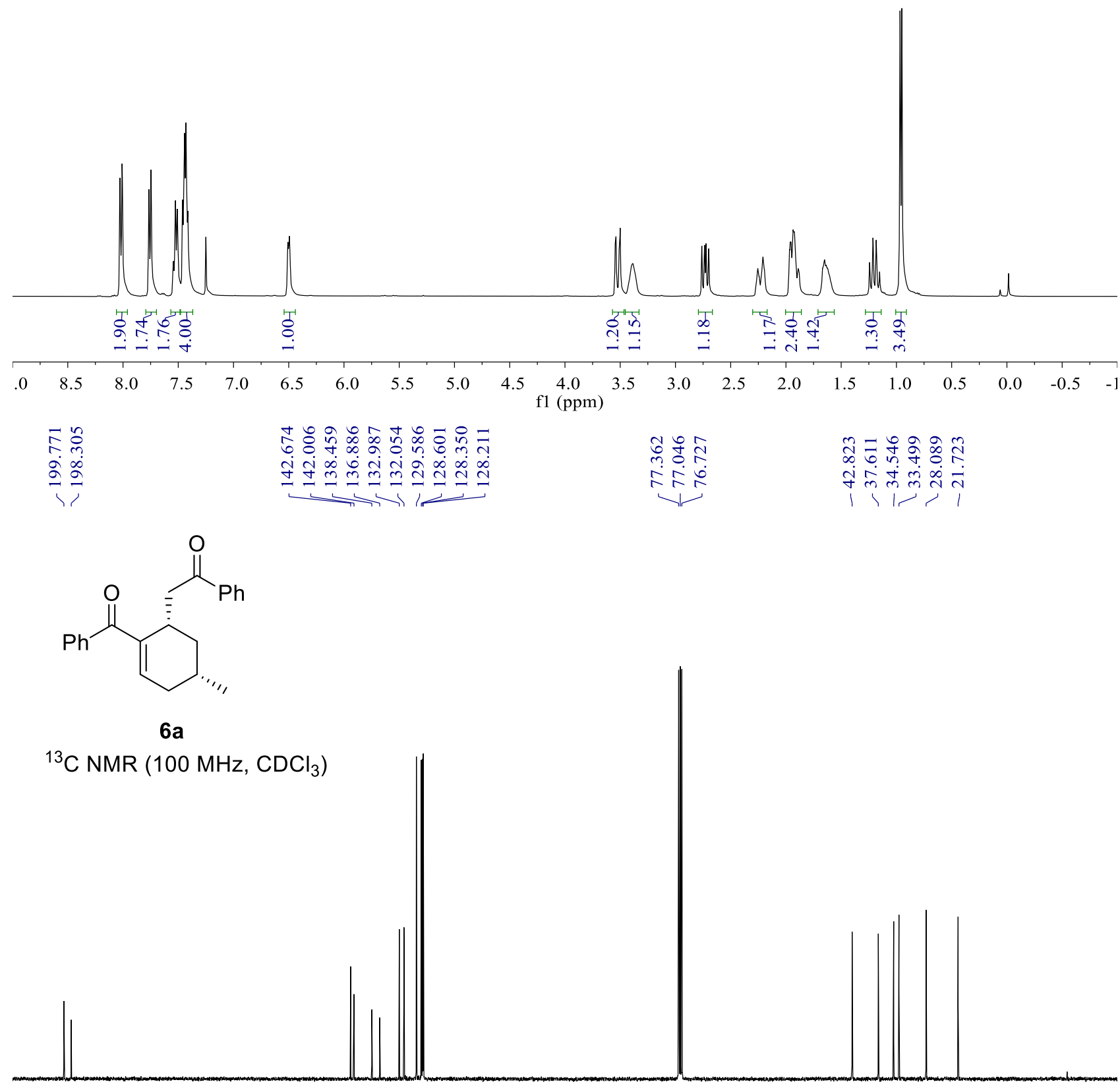

$\begin{array}{lllllllllllllllllllllll}10 & 200 & 190 & 180 & 170 & 160 & 150 & 140 & 130 & 120 & 110 \underset{\mathrm{f} 1(\mathrm{ppm})}{100} & 90 & 80 & 70 & 60 & 50 & 40 & 30 & 20 & 10 & 0 & -\end{array}$ 


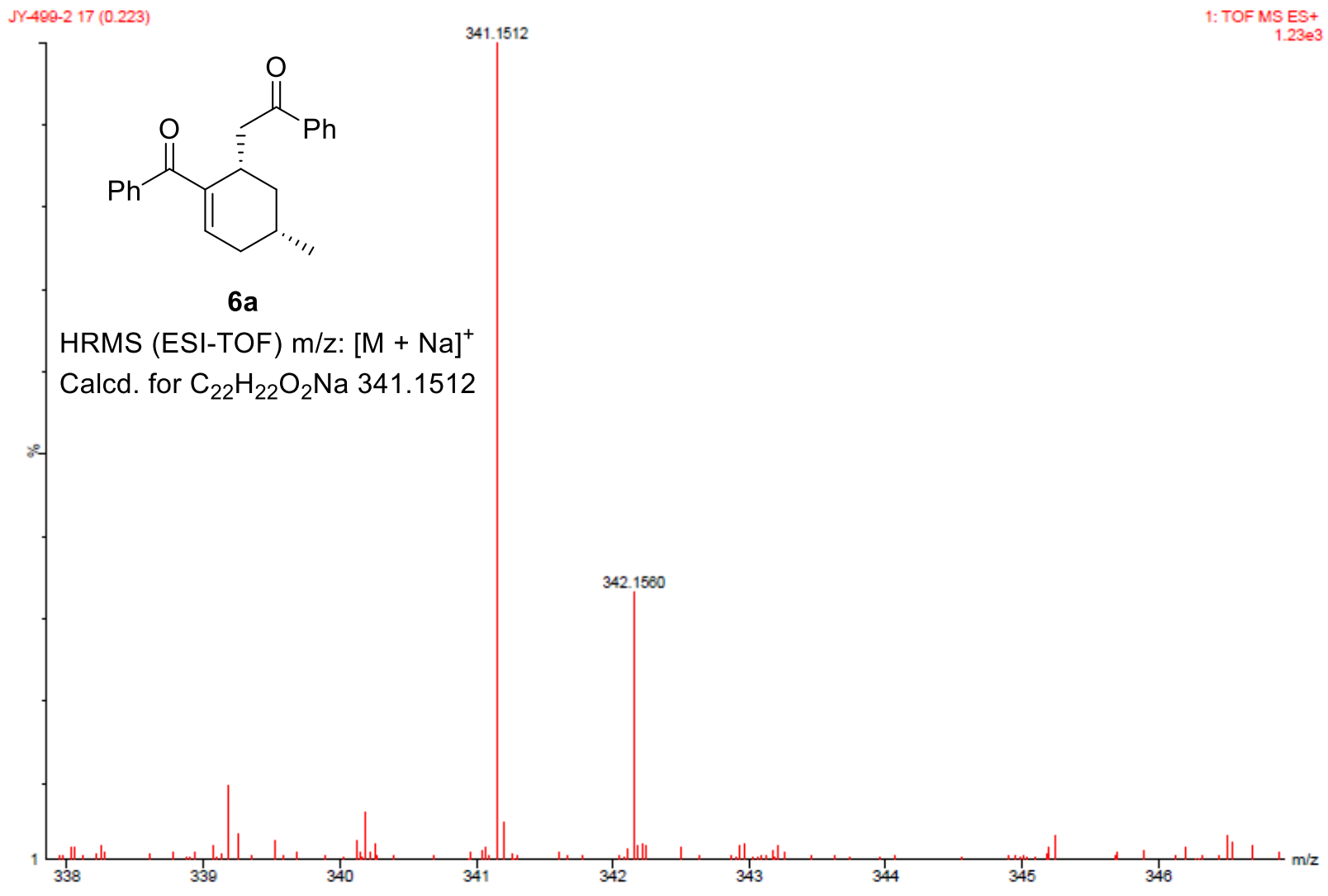




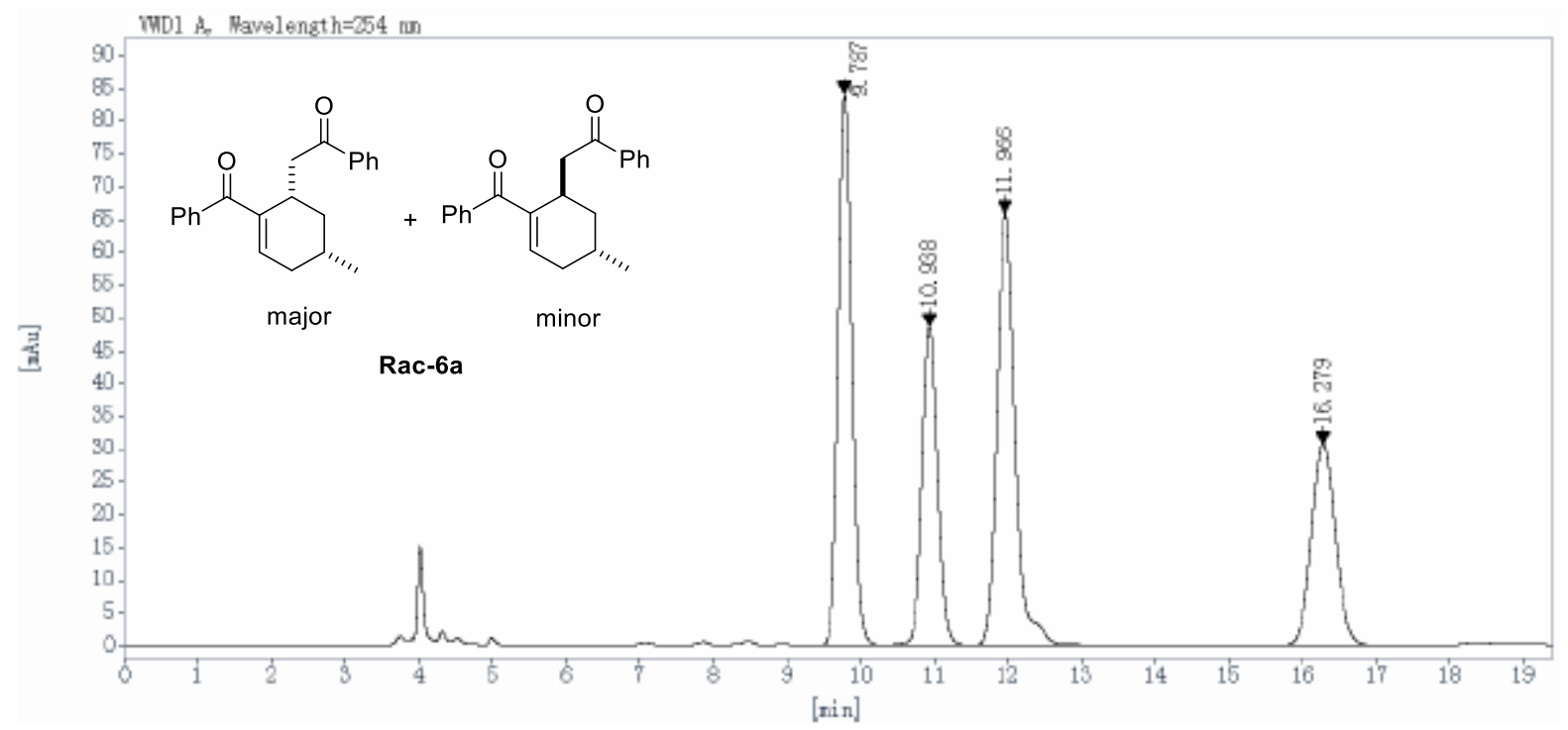

$\begin{array}{cccccc}\begin{array}{c}\text { Ret Time } \\ \text { [min] }\end{array} & \begin{array}{c}\text { Peak } \\ \text { Type }\end{array} & \begin{array}{c}\text { Width } \\ {[\mathrm{min}]}\end{array} & \begin{array}{c}\text { Height } \\ {[\mathrm{mAU} \text { ] }}\end{array} & \begin{array}{c}\text { Area } \\ {\left[\mathrm{mAU}^{*} \text { s] }\right.}\end{array} & \begin{array}{c}\text { Area } \\ {[\%]}\end{array} \\ 9.787 & \text { BB } & 0.20 & 84.2253 & 1077.4279 & 29.8186 \\ 10.938 & \text { BB } & 0.22 & 48.5765 & 701.3915 & 19.4115 \\ 11.966 & \text { BB } & 0.26 & 65.8842 & 1139.8864 & 31.5472 \\ 16.279 & \text { BB } & 0.35 & 30.7932 & 694.5646 & 19.2226 \\ & & & \text { Totals: } & 3613.2703 & 100.0000\end{array}$

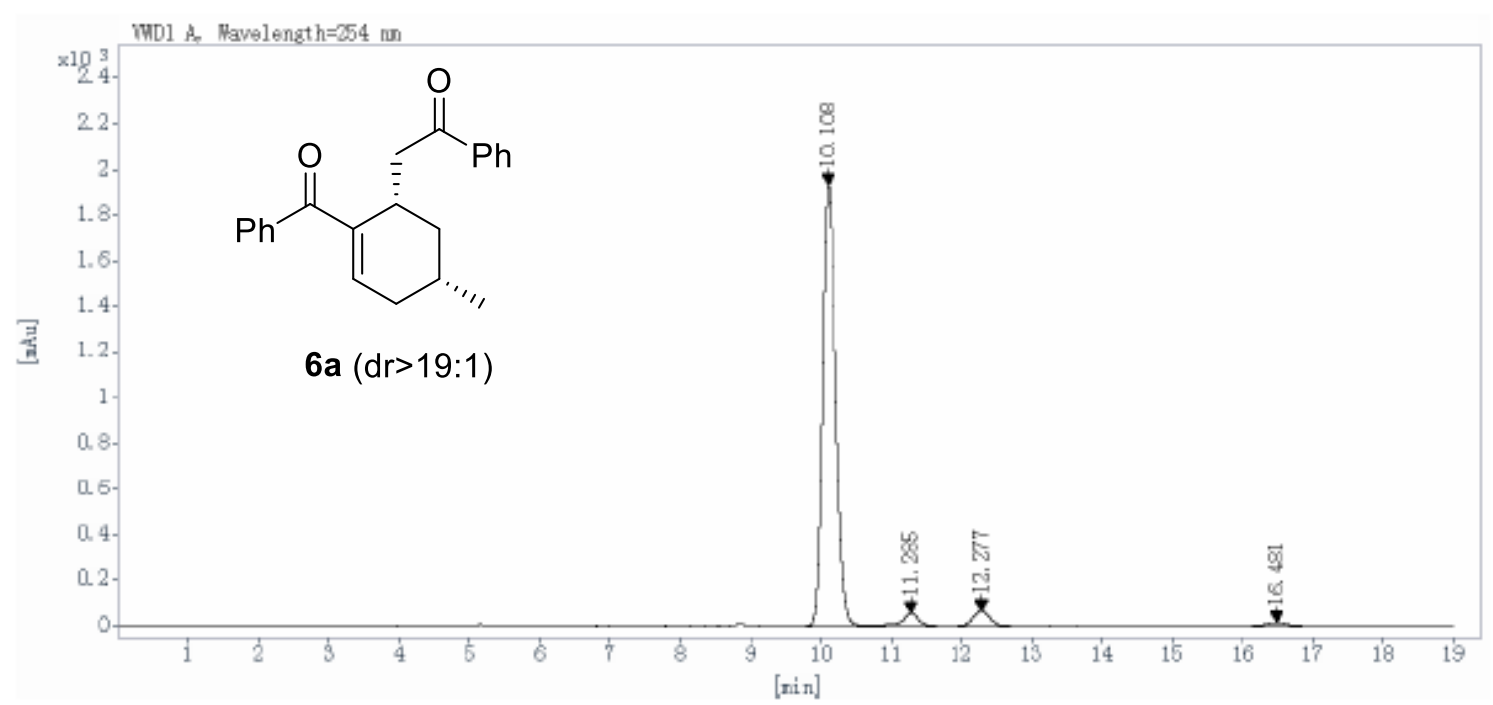

\begin{tabular}{cccccc}
$\begin{array}{c}\text { Ret Time } \\
\text { [min] }\end{array}$ & $\begin{array}{c}\text { Peak } \\
\text { Type }\end{array}$ & $\begin{array}{c}\text { Width } \\
{[\mathrm{min}]}\end{array}$ & $\begin{array}{c}\text { Height } \\
{[\mathrm{mAU}]}\end{array}$ & $\begin{array}{c}\text { Area } \\
{\left[\mathrm{mAU}^{*} \text { s] }\right.}\end{array}$ & $\begin{array}{c}\text { Area } \\
{[\%]}\end{array}$ \\
\hline 10.108 & BV & 0.20 & 1928.7958 & 25187.7773 & 91.5332 \\
11.285 & VV & 0.25 & 56.0429 & 944.5508 & 3.4325 \\
12.277 & VB & 0.25 & 65.7847 & 1071.5723 & 3.8941 \\
16.481 & BB & 0.33 & 14.6375 & 313.7310 & 1.1401 \\
& & & Totals: & 27517.6314 & 100.0000
\end{tabular}


<smiles>Cc1ccc(C(=O)C[C@H]2C[C@H](C)CC=C2C(=O)c2ccc(C)cc2)cc1</smiles>

6b

${ }^{1} \mathrm{H}$ NMR $\left(400 \mathrm{MHz}, \mathrm{CDCl}_{3}\right)$

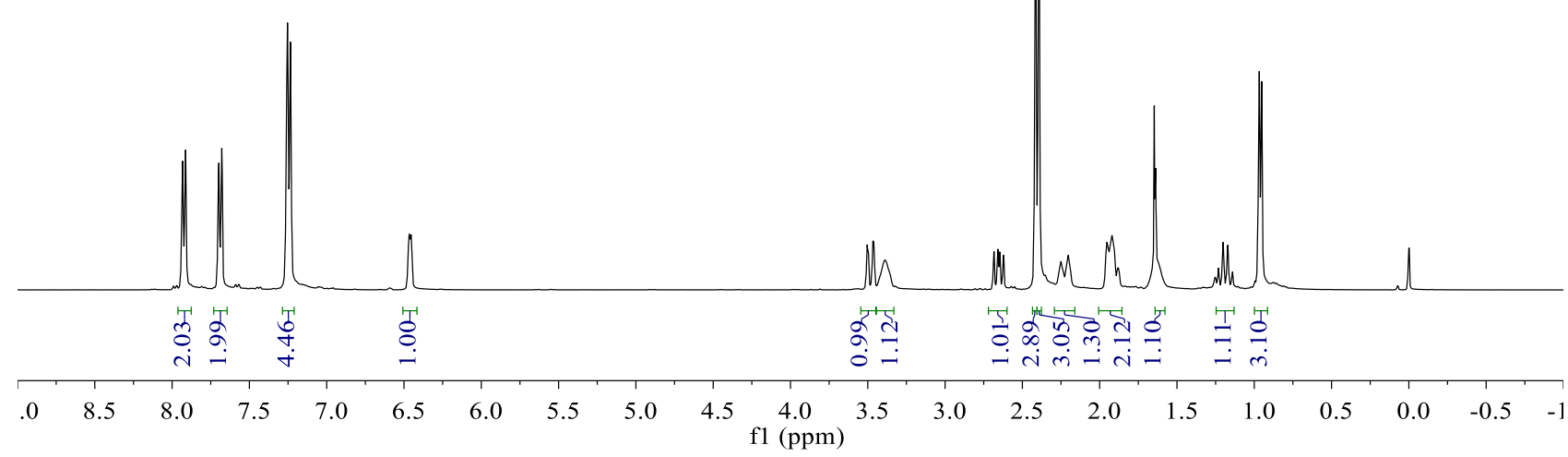

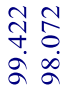

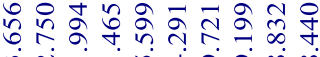

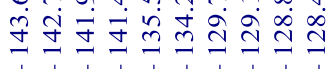

돈용

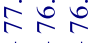

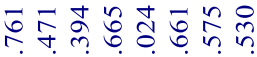

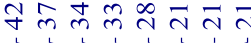<smiles>Cc1ccc(C(=O)C[C@H]2C[C@H](C)CC=C2C(=O)c2ccc(C)cc2)cc1</smiles>

6b

${ }^{13} \mathrm{C}$ NMR $\left(150 \mathrm{MHz}, \mathrm{CDCl}_{3}\right)$

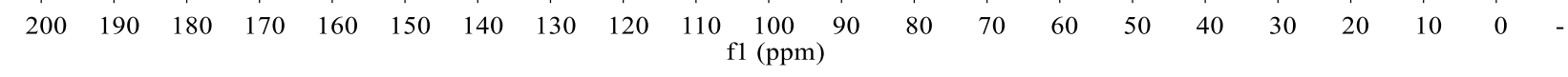



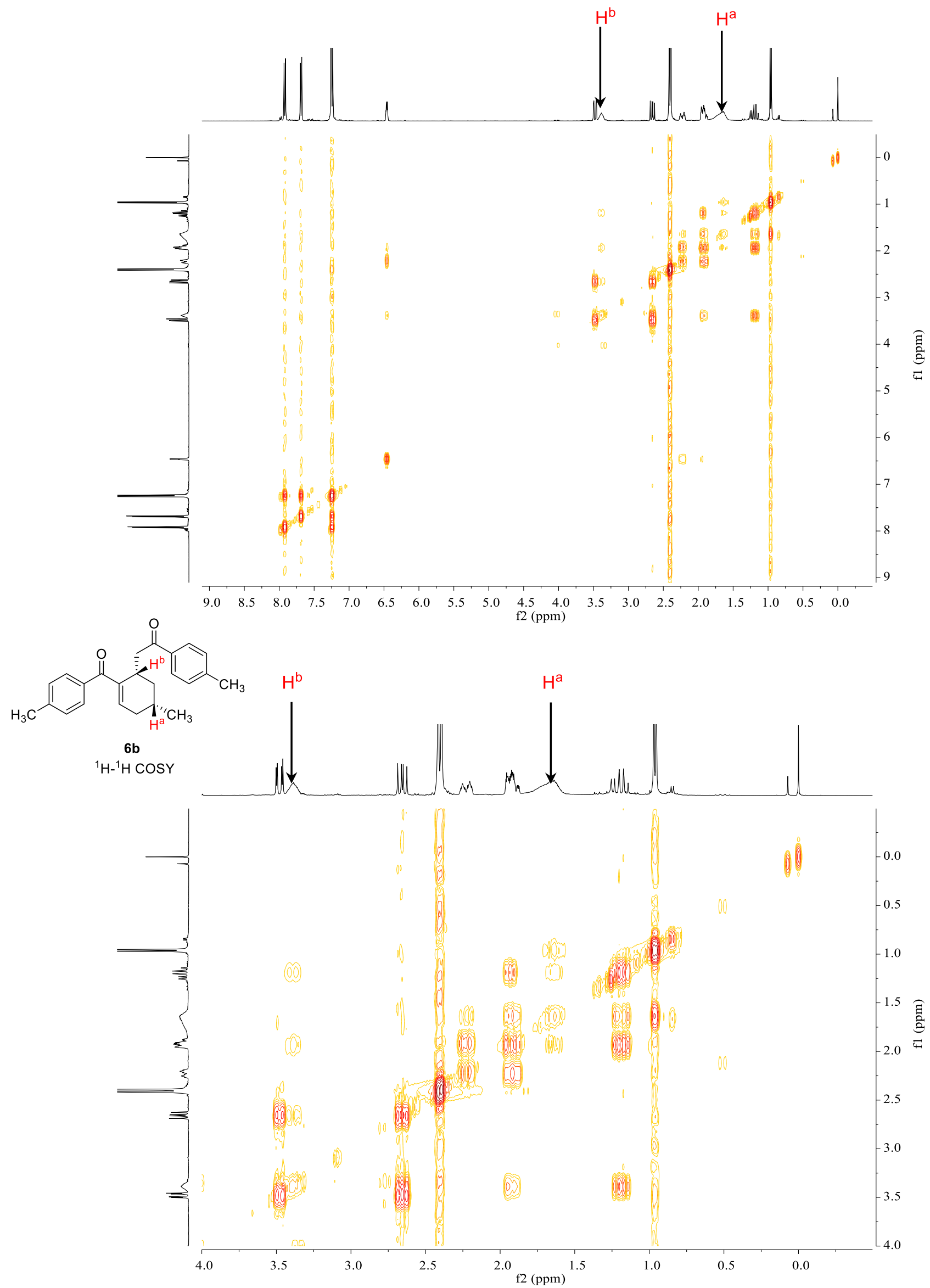
<smiles>Cc1ccc(C(=O)C[C@@H]2C[C@H](C)[C@H](C)CC=C2C(=O)c2ccc(C)cc2)cc1</smiles>

6b, NOEDS, $1.65 \mathrm{ppm}$ NOEDS NMR $\left(600 \mathrm{MHz}, \mathrm{CDCl}_{3}\right)$ $\overbrace{\substack{n \\ \infty}}^{\infty}$

$\mathrm{H}^{\mathrm{b}}$ signal at $3.38 \mathrm{ppm} \quad$ irradiation $\mathrm{H}^{\mathrm{a}}$ at $1.65 \mathrm{ppm}$

$\begin{array}{lllllllllllllllllllllllll}.0 & 8.5 & 8.0 & 7.5 & 7.0 & 6.5 & 6.0 & 5.5 & 5.0 & 4.5 & \begin{array}{l}4.0 \\ \mathrm{fl}(\mathrm{ppm})\end{array} & 3.5 & 3.0 & 2.5 & 2.0 & 1.5 & 1.0 & 0.5 & 0.0 & -0.5 & -1\end{array}$

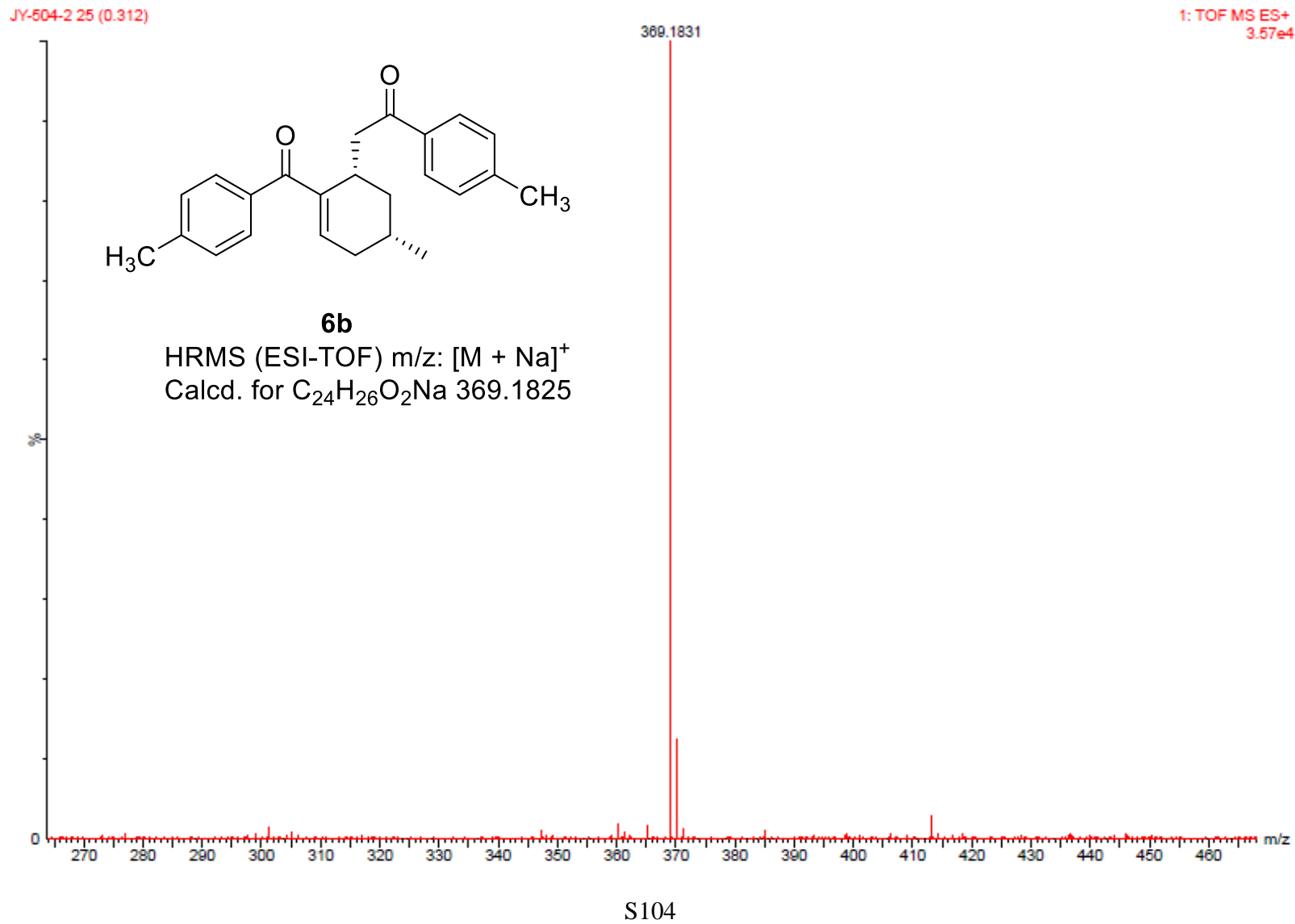




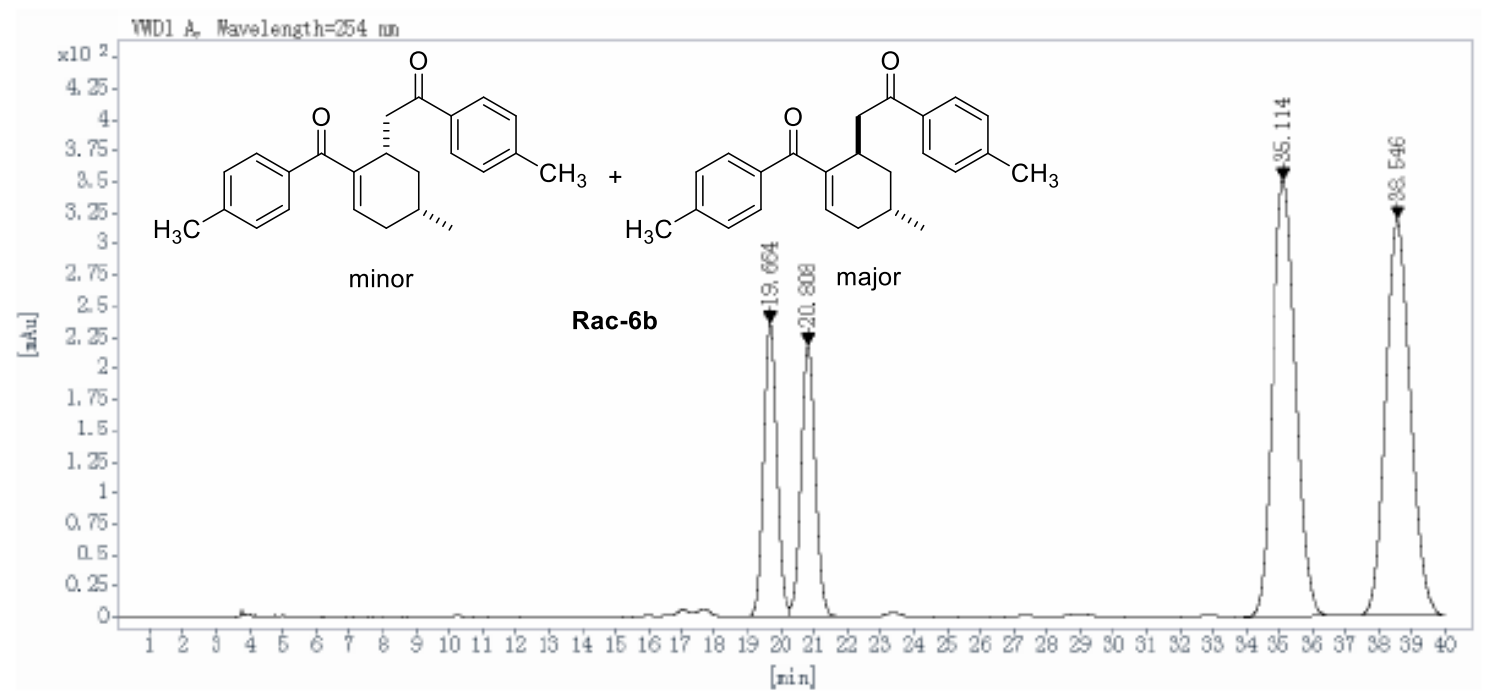

\begin{tabular}{cccccc}
$\begin{array}{c}\text { Ret Time } \\
\text { [min] }\end{array}$ & $\begin{array}{c}\text { Peak } \\
\text { Type }\end{array}$ & $\begin{array}{c}\text { Width } \\
{[\min ]}\end{array}$ & $\begin{array}{c}\text { Height } \\
{[\mathrm{mAU}]}\end{array}$ & $\begin{array}{c}\text { Area } \\
{\left[\mathrm{mAU}^{*} \text { s] }\right.}\end{array}$ & $\begin{array}{c}\text { Area } \\
{[\%]}\end{array}$ \\
\hline 19.664 & BV & 0.42 & 236.1750 & 6368.9492 & 13.8042 \\
20.808 & VBA & 0.45 & 218.5352 & 6326.0317 & 13.7112 \\
35.114 & BB & 0.74 & 350.9660 & 16756.9688 & 36.3195 \\
38.546 & BBA & 0.81 & 318.8164 & 16685.6582 & 36.1650 \\
& & & Totals: & 46137.6079 & 100.0000
\end{tabular}

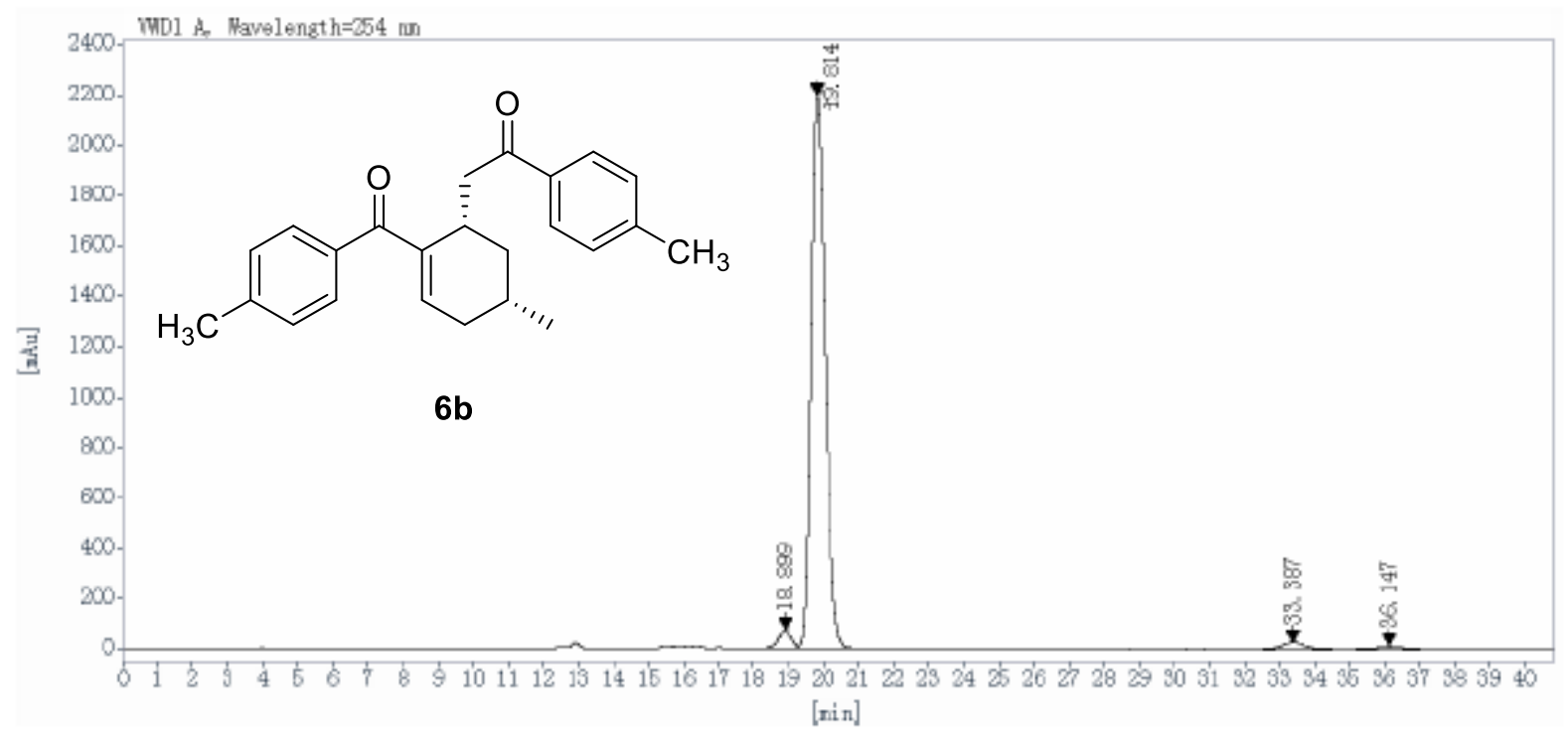

$\begin{array}{cccccc}\begin{array}{c}\text { Ret Time } \\ \text { [min] }\end{array} & \begin{array}{c}\text { Peak } \\ \text { Type }\end{array} & \begin{array}{c}\text { Width } \\ {[\mathrm{min}]}\end{array} & \begin{array}{c}\text { Height } \\ {[\mathrm{mAU}]}\end{array} & \begin{array}{c}\text { Area } \\ {[\mathrm{mAU} \text { *s] }}\end{array} & \begin{array}{c}\text { Area } \\ {[\%]}\end{array} \\ 18.899 & \text { BV E } & 0.41 & 73.3223 & 1942.2263 & 3.0156 \\ 19.814 & \text { VB R } & 0.43 & 2198.5022 & 60725.8555 & 94.2856 \\ 33.387 & \text { BB } & 0.70 & 27.3037 & 1219.6494 & 1.8937 \\ 36.147 & \text { BB } & 0.75 & 10.4866 & 518.5704 & 0.8052 \\ & & & \text { Totals: } & 64406.3016 & 100.0000\end{array}$


<smiles>COc1cccc(C(=O)C[C@H]2C[C@H](C)CC=C2C(=O)c2cccc(OC)c2)c1</smiles>

$6 c$

${ }^{1} \mathrm{H} \mathrm{NMR}\left(400 \mathrm{MHz}, \mathrm{CDCl}_{3}\right)$

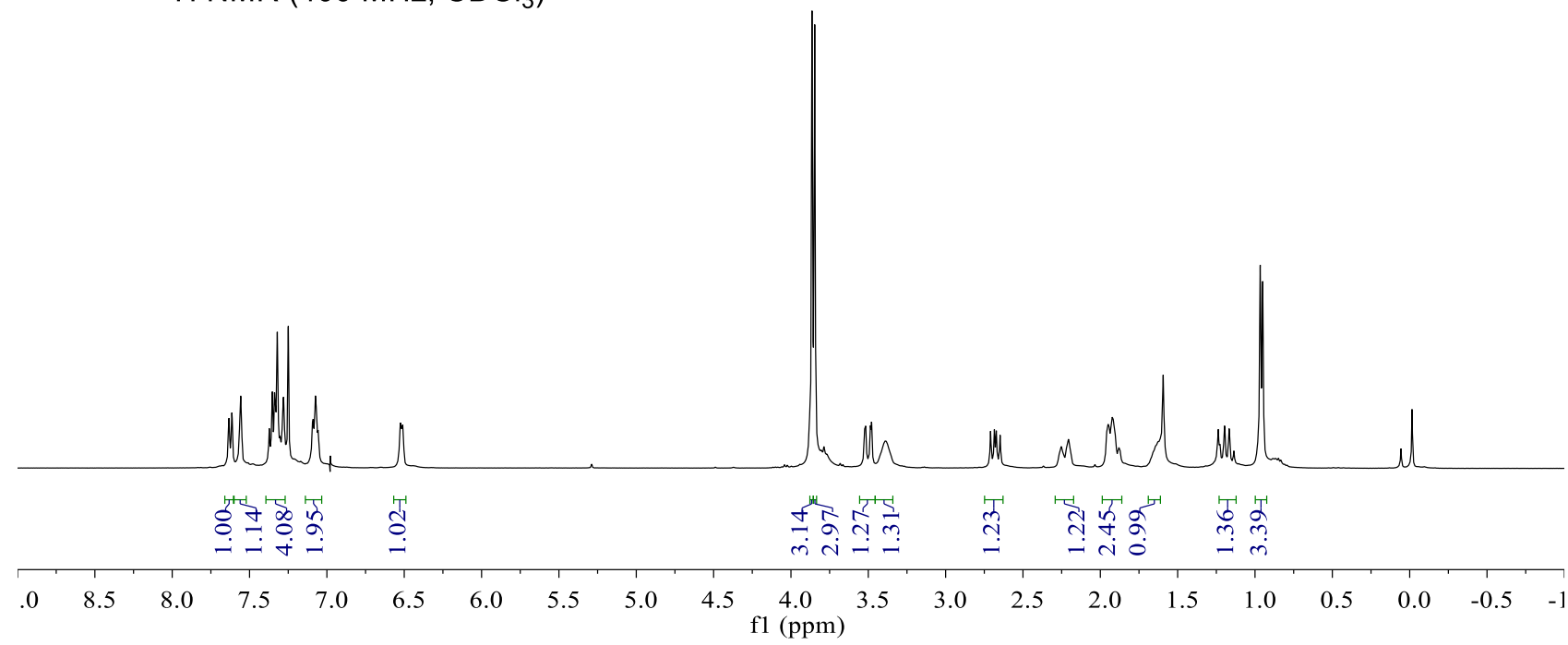

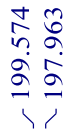

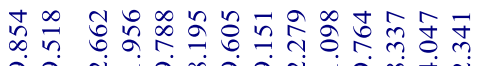

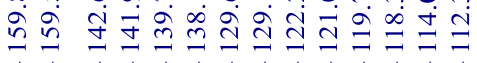

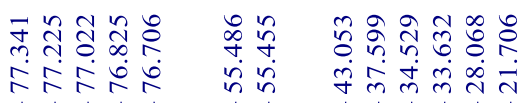

(1) ${ }^{\mathrm{OCH}_{3}}$

$6 c$

${ }^{13} \mathrm{C}$ NMR $\left(100 \mathrm{MHz}, \mathrm{CDCl}_{3}\right)$

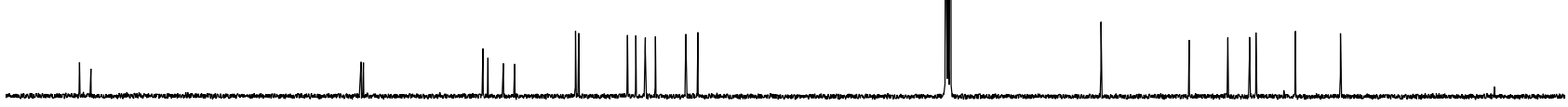

$\begin{array}{lllllllllllllllllllllll}10 & 200 & 190 & 180 & 170 & 160 & 150 & 140 & 130 & 120 & 110 \underset{\mathrm{f} 1 \mathrm{(ppm})}{100} & 90 & 80 & 70 & 60 & 50 & 40 & 30 & 20 & 10 & 0 & -\end{array}$ 


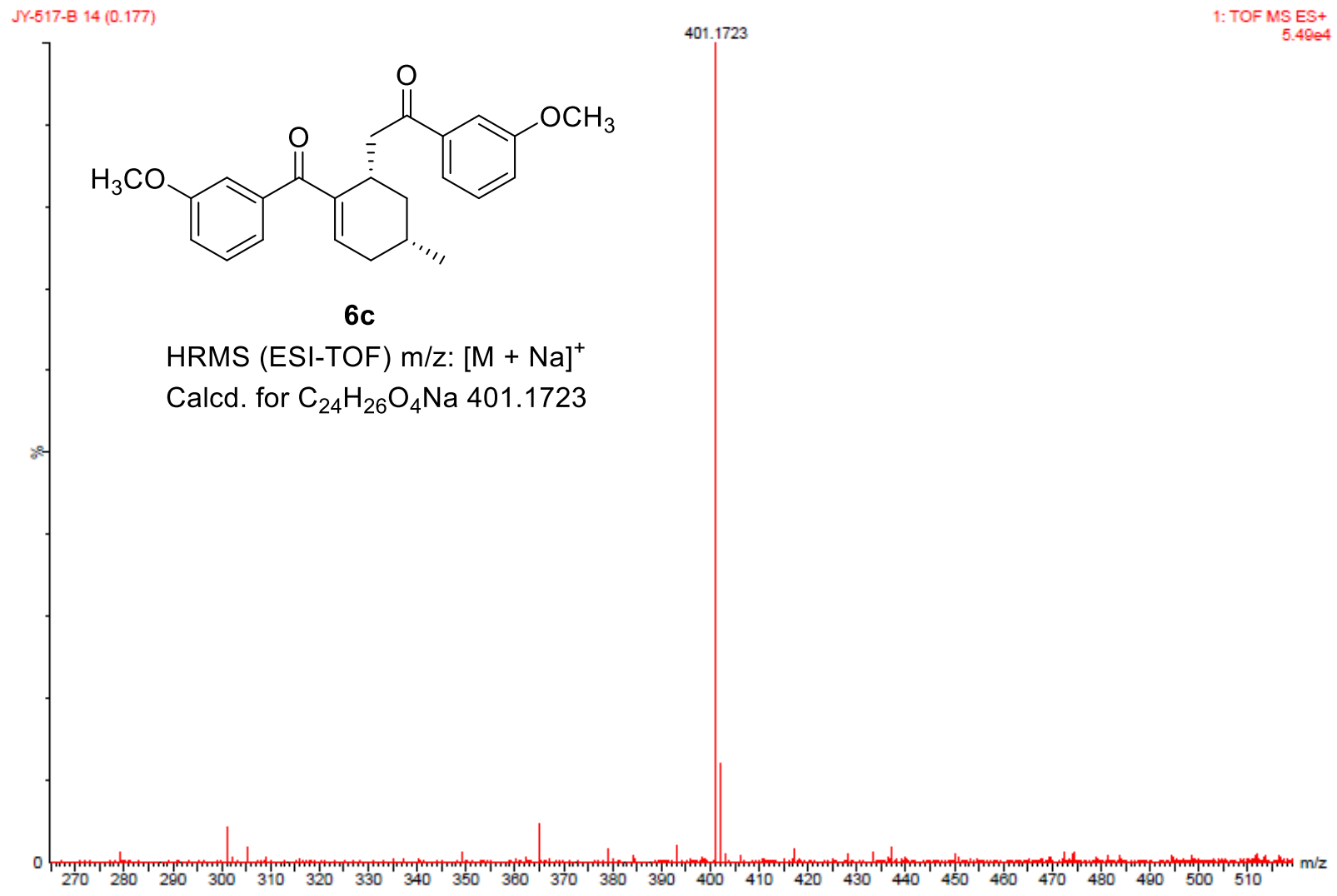




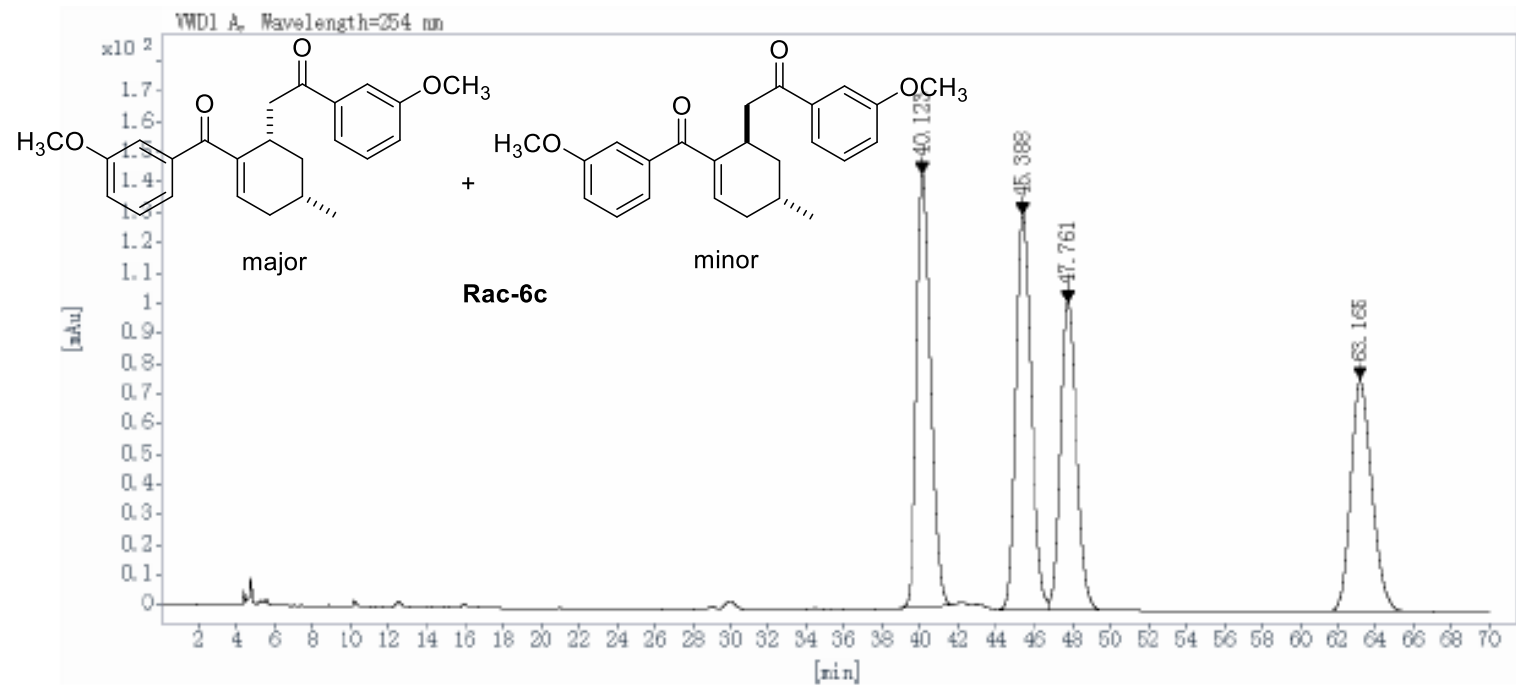

\begin{tabular}{cccccc}
$\begin{array}{c}\text { Ret Time } \\
\text { [min] }\end{array}$ & $\begin{array}{c}\text { Peak } \\
\text { Type }\end{array}$ & $\begin{array}{c}\text { Width } \\
{[\text { min] }}\end{array}$ & $\begin{array}{c}\text { Height } \\
{[\mathrm{mAU}]}\end{array}$ & $\begin{array}{c}\text { Area } \\
{[\mathrm{mAU} * \text { s] }}\end{array}$ & $\begin{array}{c}\text { Area } \\
{[\%]}\end{array}$ \\
\hline 40.123 & BB & 0.79 & 143.6912 & 7370.5063 & 27.4011 \\
45.388 & BV & 0.87 & 131.2820 & 7390.3960 & 27.4750 \\
47.761 & VB & 0.92 & 102.4068 & 6059.8892 & 22.5287 \\
63.165 & BB & 1.21 & 77.2503 & 6077.7827 & 22.5952 \\
& & & Totals: & 26898.5742 & 100.0000
\end{tabular}

TMDI A. Mavelength $=254$ in

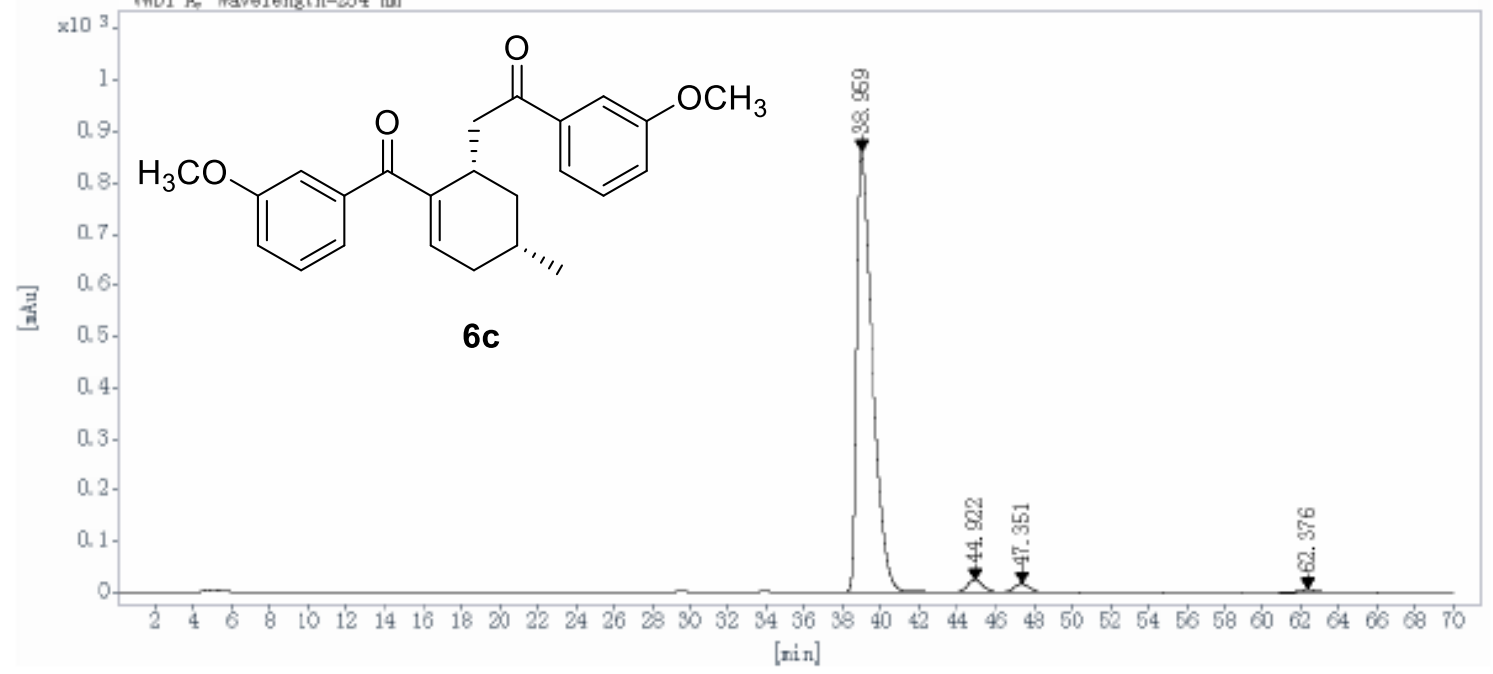

$\begin{array}{cccccc}\begin{array}{c}\text { Ret Time } \\ \text { [min] }\end{array} & \begin{array}{c}\text { Peak } \\ \text { Type }\end{array} & \begin{array}{c}\text { Width } \\ {[\mathrm{min}]}\end{array} & \begin{array}{c}\text { Height } \\ \text { [mAU] }\end{array} & \begin{array}{c}\text { Area } \\ {[\mathrm{mAU} \text { *s] }}\end{array} & \begin{array}{c}\text { Area } \\ {[\%]}\end{array} \\ 38.959 & \text { BB } & 0.86 & 860.5450 & 49967.3242 & 94.9275 \\ 44.922 & \text { BB } & 0.85 & 24.0588 & 1330.1923 & 2.5271 \\ 47.351 & \text { BB } & 0.89 & 16.1497 & 932.0208 & 1.7706 \\ 62.376 & \text { BB } & 1.12 & 5.1044 & 407.8092 & 0.7748 \\ & & & \text { Totals: } & 52637.3464 & 100.0000\end{array}$


<smiles>C[C@H]1CC=C(C(=O)c2ccc(Br)cc2)[C@H](CC(=O)c2ccc(Br)cc2)C1</smiles>

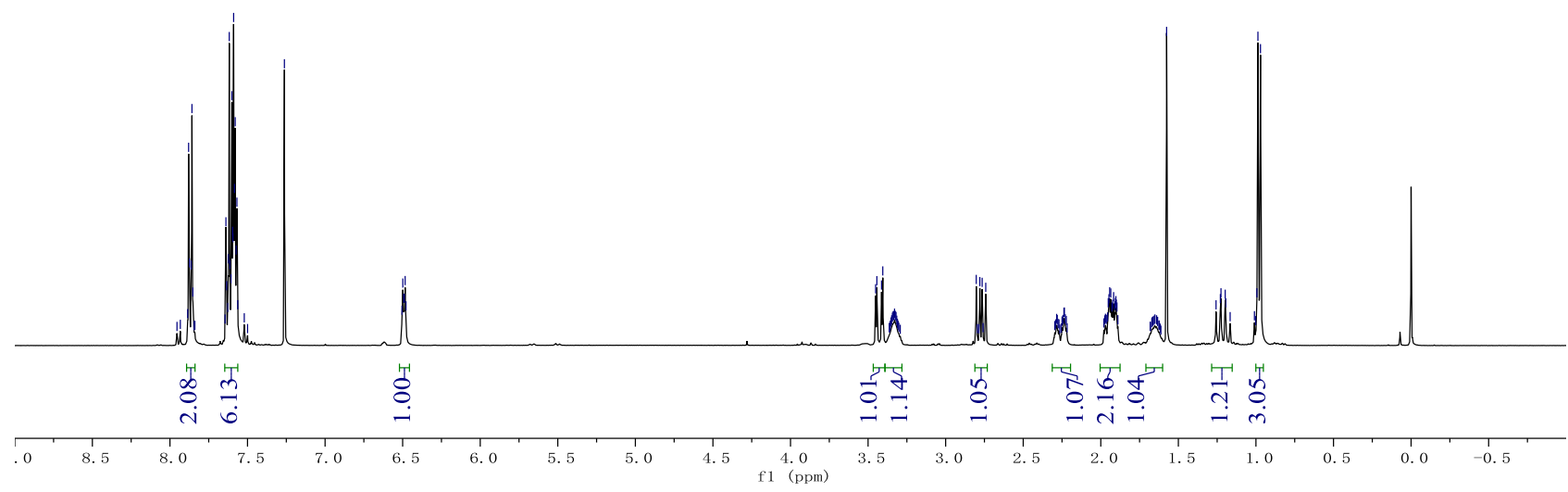

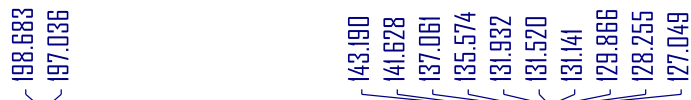<smiles>C[C@H]1CC=C(C(=O)c2ccc(Br)cc2)[C@H](CC(=O)c2ccc(Br)cc2)C1</smiles>

${ }^{13} \mathrm{C} \mathrm{NMR}\left(100 \mathrm{MHz}, \mathrm{CDCl}_{3}\right)$

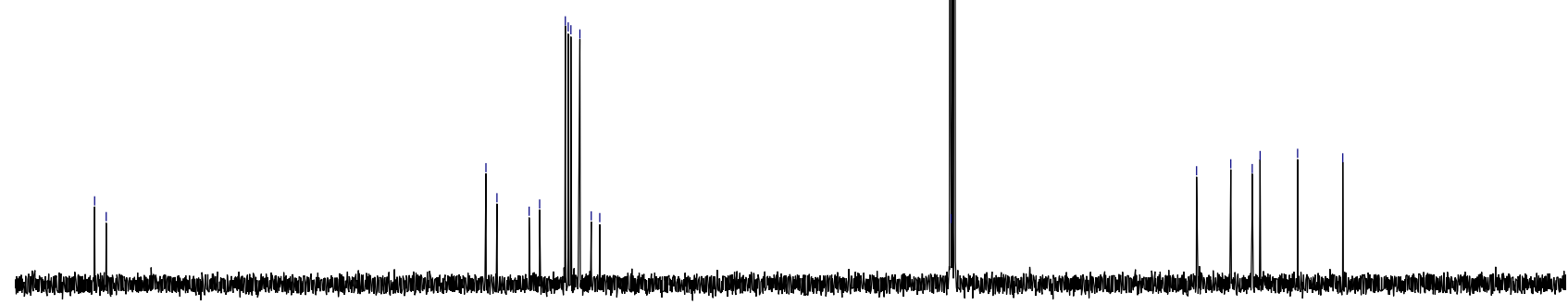




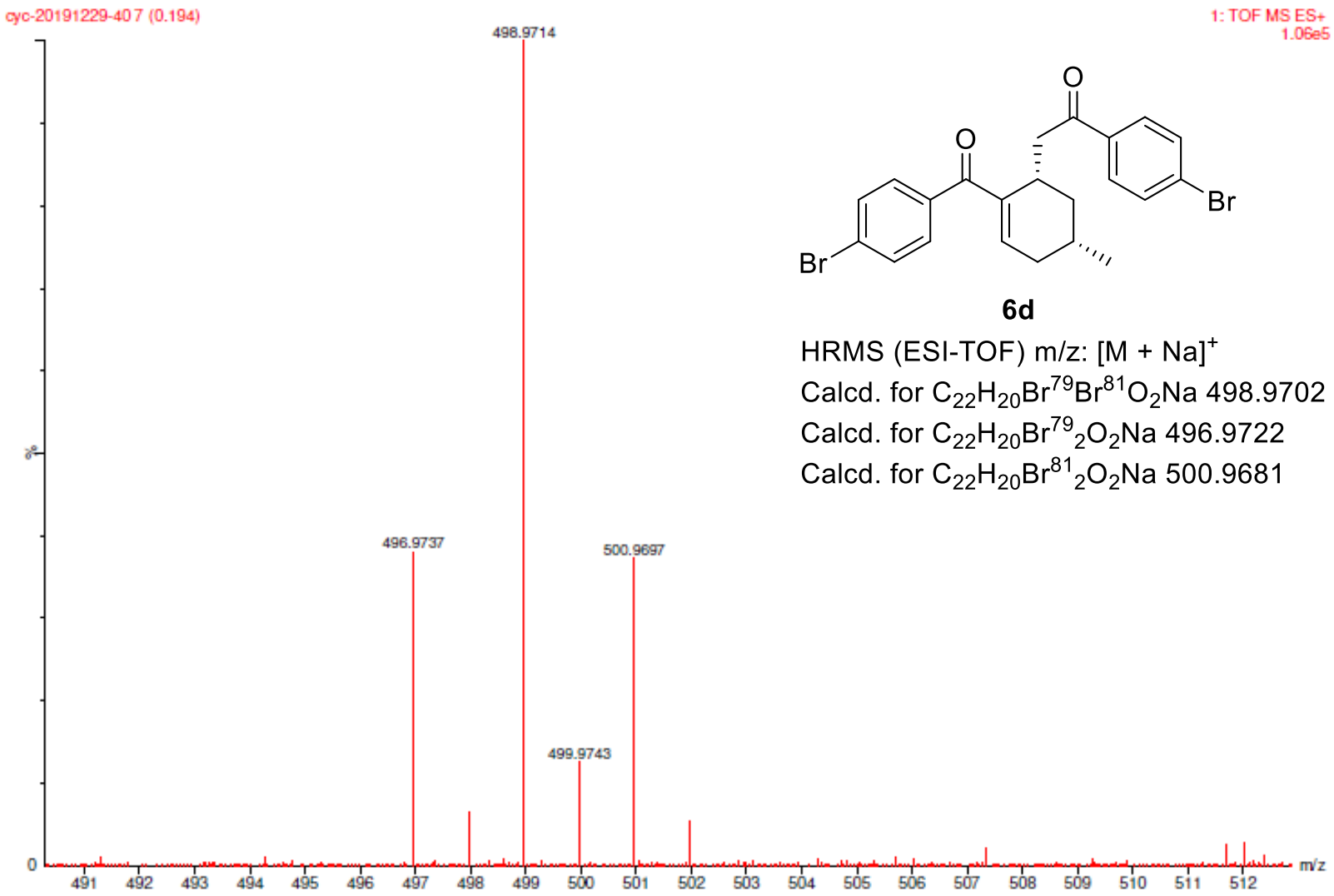




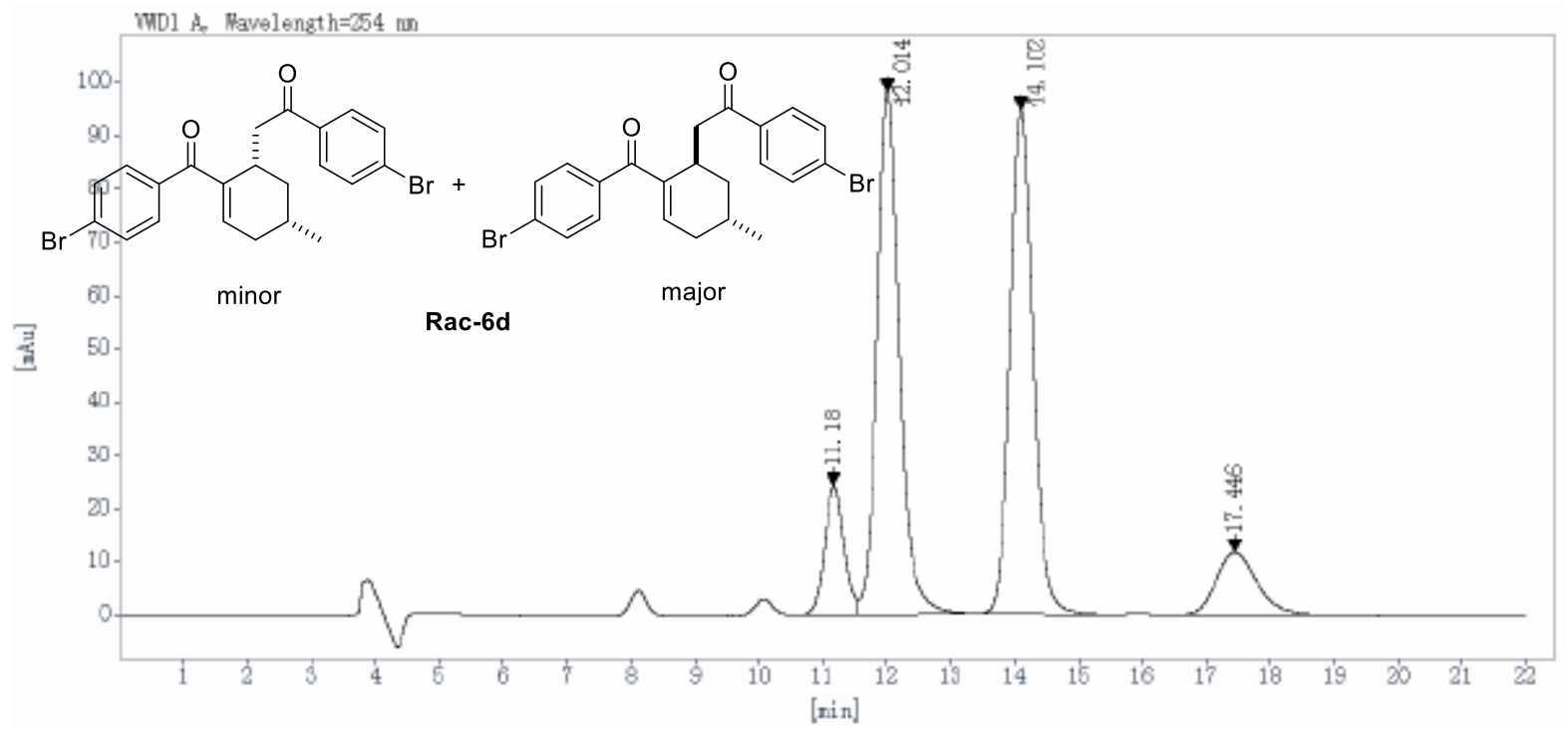

$\begin{array}{cccccc}\begin{array}{c}\text { Ret Time } \\ \text { [min] }\end{array} & \begin{array}{c}\text { Peak } \\ \text { Type }\end{array} & \begin{array}{c}\text { Width } \\ \text { [min] }\end{array} & \begin{array}{c}\text { Height } \\ \text { [mAU] }\end{array} & \begin{array}{c}\text { Area } \\ \text { [mAU*'s] }\end{array} & \begin{array}{c}\text { Area } \\ {[\%]}\end{array} \\ 11.180 & \text { BV } & 0.32 & 24.2016 & 511.6358 & 8.8905 \\ 12.014 & \text { VB } & 0.37 & 98.2640 & 2372.9534 & 41.2338 \\ 14.102 & \text { BB } & 0.38 & 94.5632 & 2349.8860 & 40.8330 \\ 17.446 & \text { BB } & 0.68 & 11.7463 & 520.3931 & 9.0427 \\ & & & \text { Totals: } & 5754.8683 & 100.0000\end{array}$

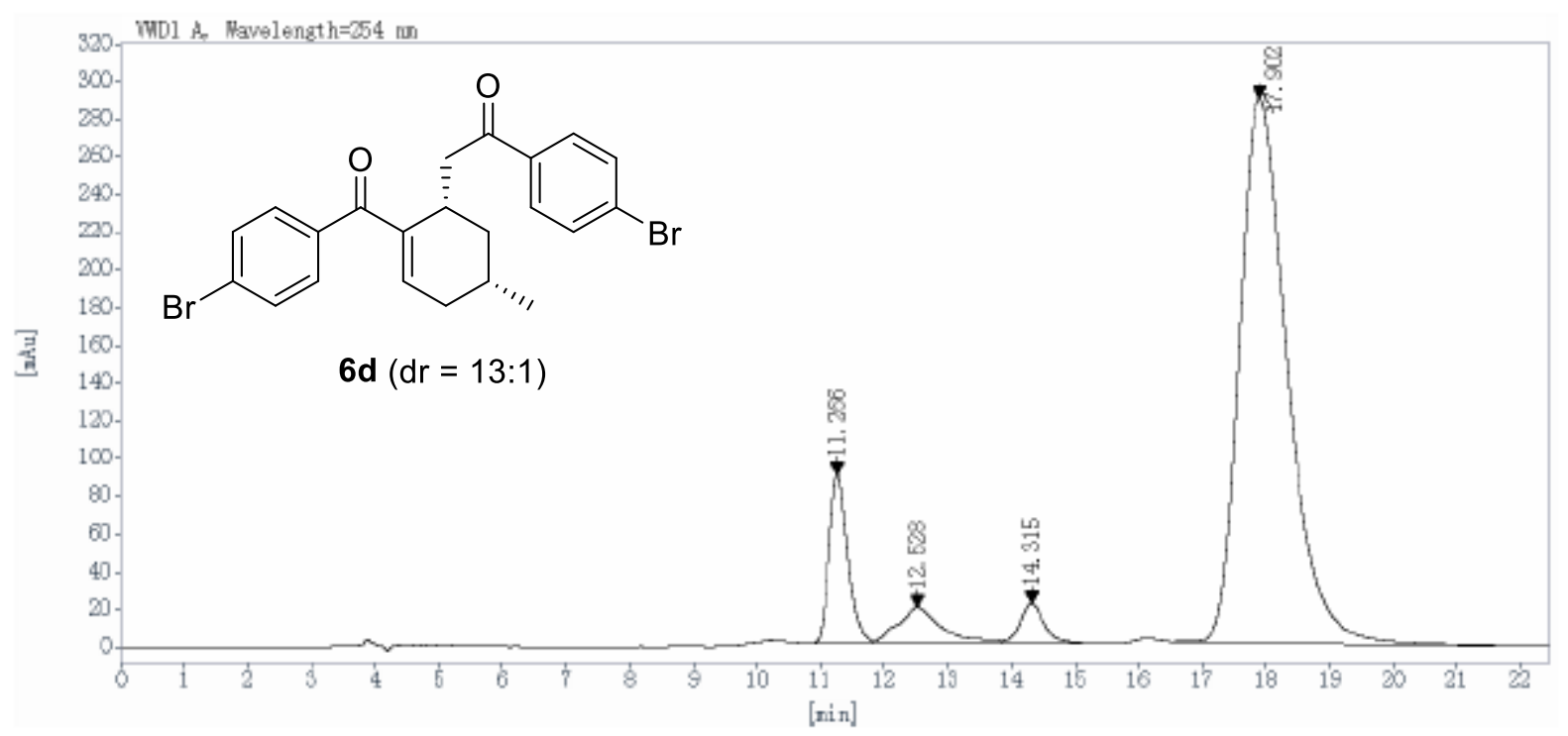

\begin{tabular}{cccccc}
$\begin{array}{c}\text { Ret Time } \\
\text { [min] }\end{array}$ & $\begin{array}{c}\text { Peak } \\
\text { Type }\end{array}$ & $\begin{array}{c}\text { Width } \\
{[\mathrm{min}]}\end{array}$ & $\begin{array}{c}\text { Height } \\
{[\mathrm{mAU}]}\end{array}$ & $\begin{array}{c}\text { Area } \\
{[\mathrm{mAU} * \text { s] }}\end{array}$ & $\begin{array}{c}\text { Area } \\
{[\%]}\end{array}$ \\
\hline 11.266 & BV & 0.31 & 89.8604 & 1824.8861 & 9.9034 \\
12.528 & VV & 0.68 & 19.2539 & 954.9671 & 5.1825 \\
14.315 & VB & 0.41 & 21.4937 & 588.3895 & 3.1931 \\
17.902 & BB & 0.80 & 289.0847 & 15058.6768 & 81.7211 \\
& & & Totals: & 18426.9195 & 100.0000
\end{tabular}




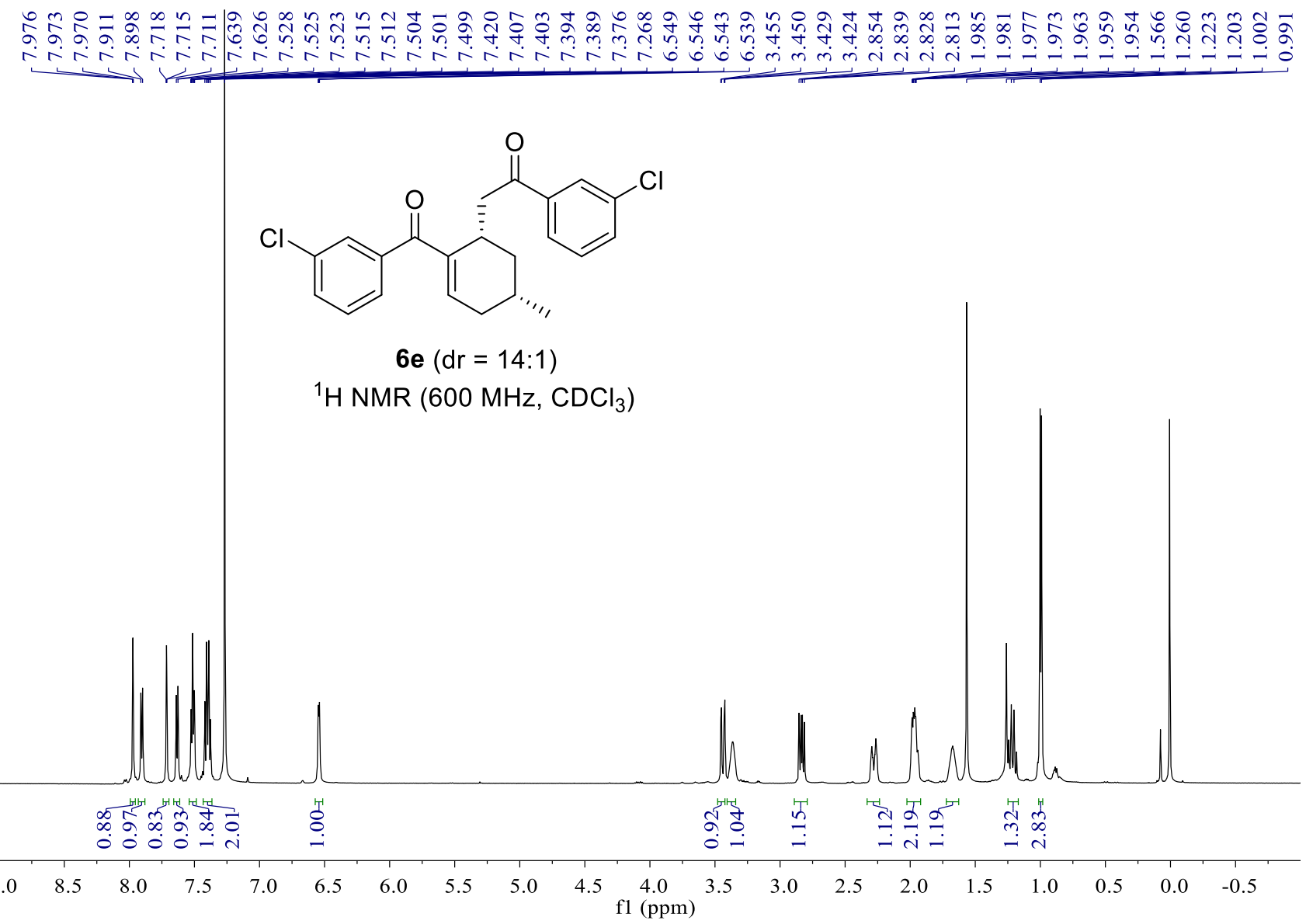<smiles>C[C@H]1CC=C(C(=O)c2cccc(Cl)c2)[C@H](CC(=O)c2cccc(Cl)c2)C1</smiles>

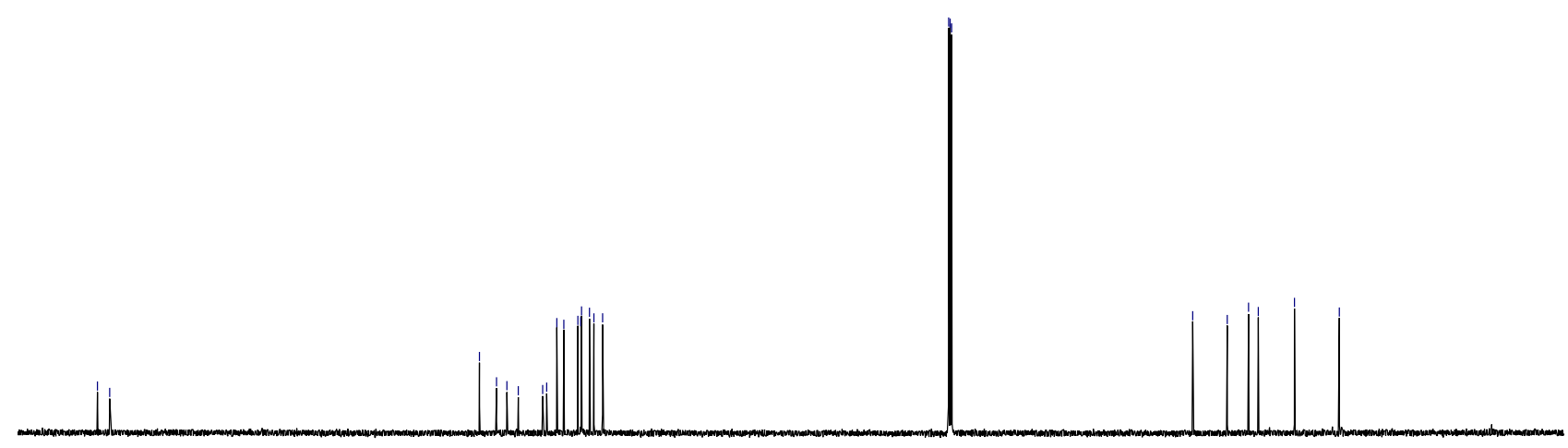

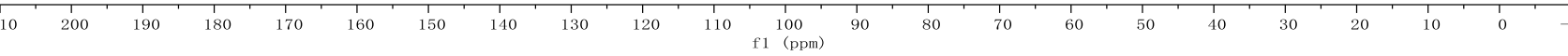




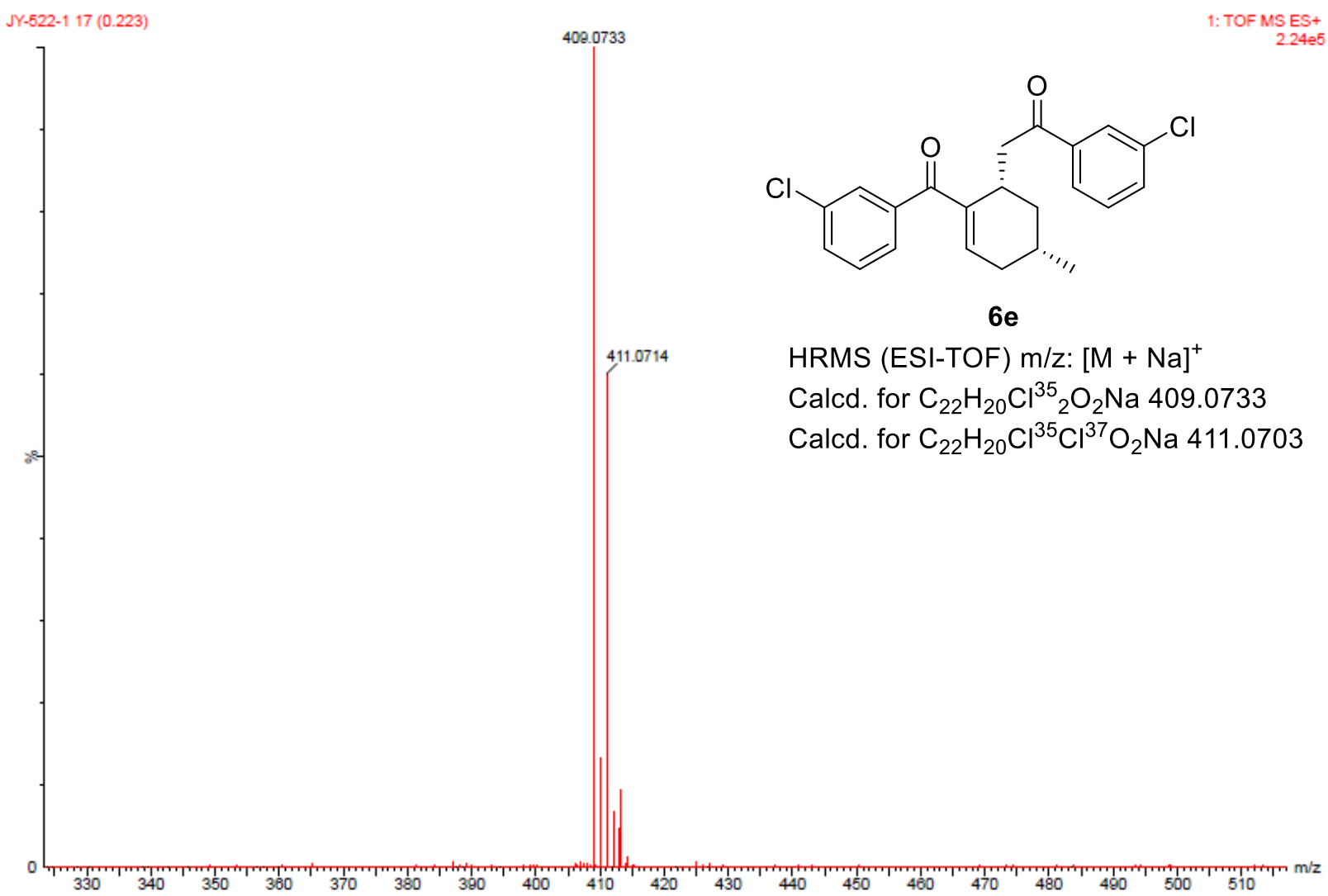


MMDI As Mavelength=254 III

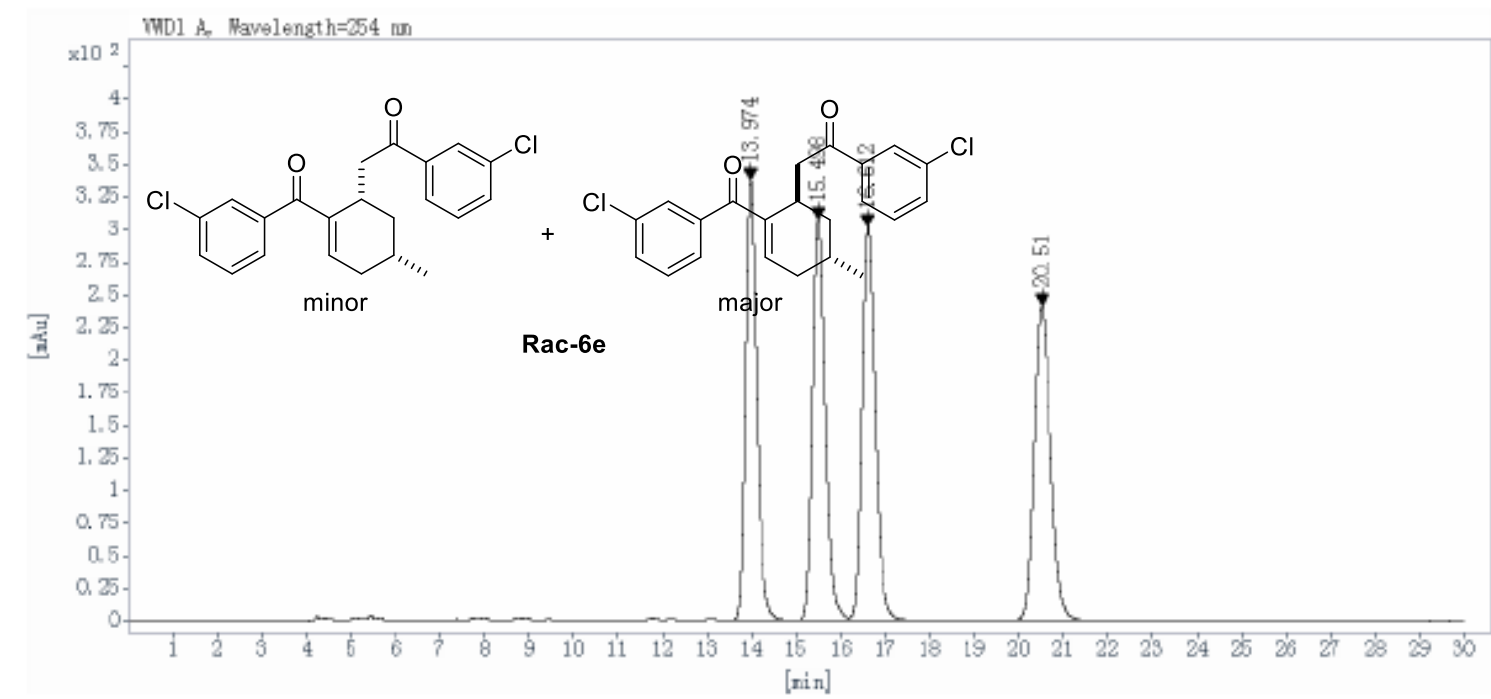

\begin{tabular}{cccccc}
$\begin{array}{c}\text { Ret Time } \\
\text { [min] }\end{array}$ & $\begin{array}{c}\text { Peak } \\
\text { Type }\end{array}$ & $\begin{array}{c}\text { Width } \\
{[\mathrm{min}]}\end{array}$ & $\begin{array}{c}\text { Height } \\
{[\mathrm{mAU}]}\end{array}$ & $\begin{array}{c}\text { Area } \\
{\left[\mathrm{mAU} \mathbf{A}^{*}\right]}\end{array}$ & $\begin{array}{c}\text { Area } \\
{[\%]}\end{array}$ \\
\hline 13.974 & BB & 0.27 & 337.4729 & 5909.9653 & 24.3999 \\
15.498 & BV & 0.30 & 307.5271 & 5955.8574 & 24.5893 \\
16.612 & VB & 0.32 & 302.0124 & 6175.1792 & 25.4948 \\
20.510 & BB & 0.40 & 241.1024 & 6180.3086 & 25.5160 \\
& & & Totals: & 24221.3105 & 100.0000
\end{tabular}

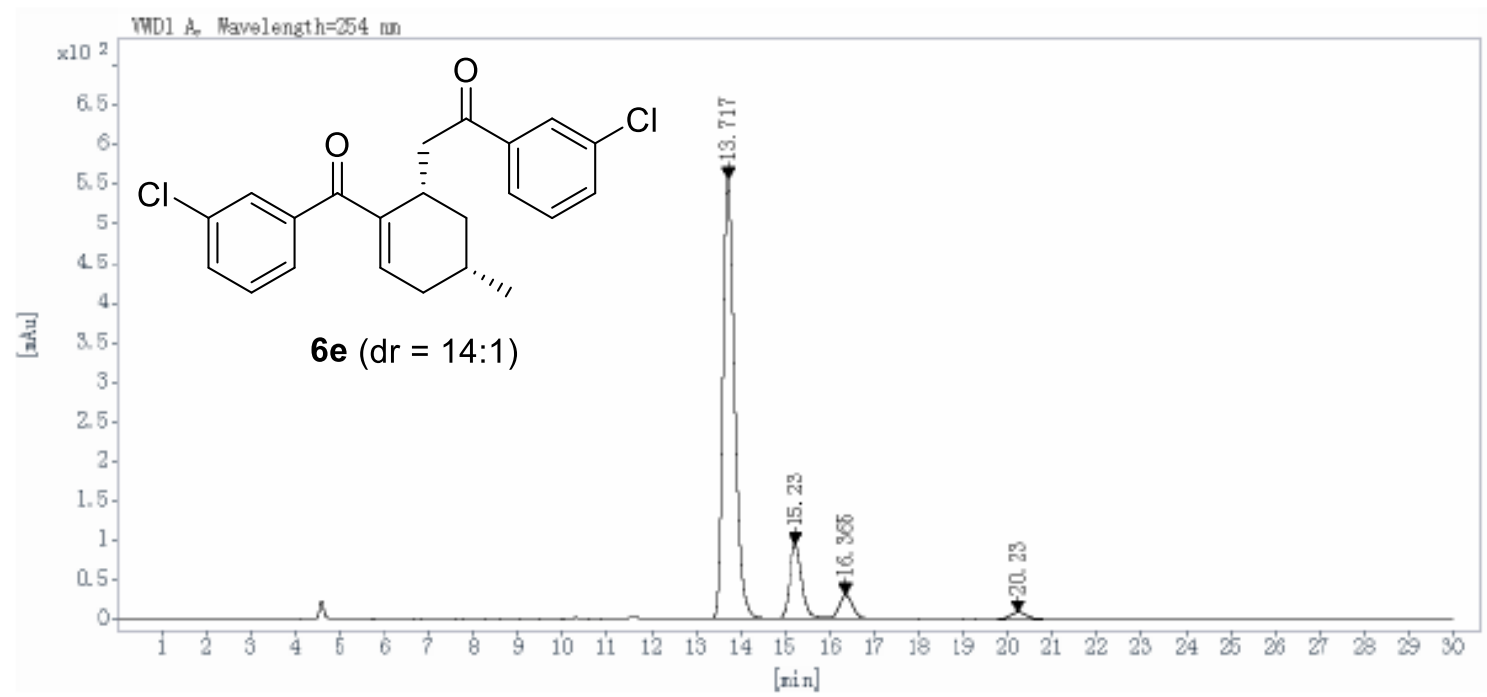

\begin{tabular}{cccccc}
$\begin{array}{c}\text { Ret Time } \\
\text { [min] }\end{array}$ & $\begin{array}{c}\text { Peak } \\
\text { Type }\end{array}$ & $\begin{array}{c}\text { Width } \\
{[\mathrm{min}]}\end{array}$ & $\begin{array}{c}\text { Height } \\
{[\mathrm{mAU}]}\end{array}$ & $\begin{array}{c}\text { Area } \\
{\left[\mathrm{mAU} *_{\text {s }}\right]}\end{array}$ & $\begin{array}{c}\text { Area } \\
{[\%]}\end{array}$ \\
\hline 13.717 & BB & 0.27 & 555.8606 & 9752.9385 & 78.7328 \\
15.230 & BV & 0.29 & 94.4202 & 1811.9177 & 14.6271 \\
16.365 & VB & 0.31 & 30.4685 & 609.2589 & 4.9184 \\
20.230 & BB & 0.39 & 8.5408 & 213.2726 & 1.7217 \\
& & & Totals: & 12387.3877 & 100.0000
\end{tabular}


<smiles>C[C@H]1CC=C(C(=O)c2ccc3ccccc3c2)[C@H](CC(=O)c2ccc3ccccc3c2)C1</smiles>

$6 f$

${ }^{1} \mathrm{H}$ NMR (400 MHz, $\left.\mathrm{CDCl}_{3}\right)$

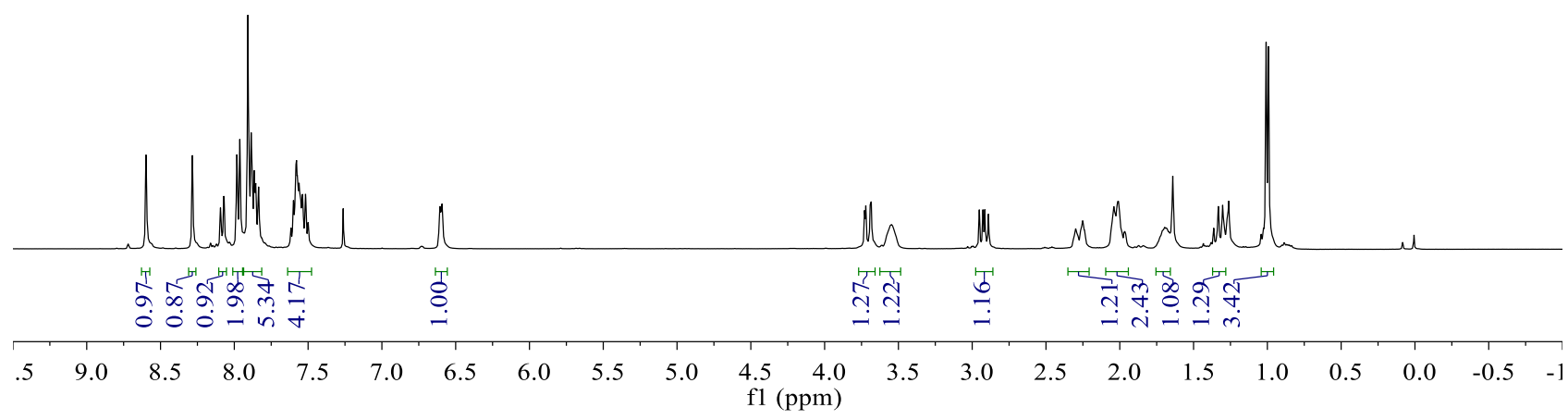<smiles>C[C@H]1CC=C(C(=O)c2ccc3ccccc3c2)[C@H](CC(=O)c2ccc3ccccc3c2)C1</smiles>

$6 f$

${ }^{13} \mathrm{C}$ NMR $\left(100 \mathrm{MHz}, \mathrm{CDCl}_{3}\right)$

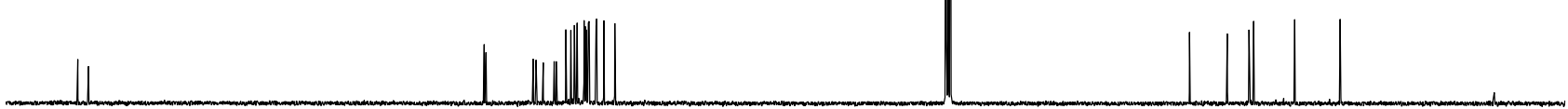

$\begin{array}{llllllllllllllllllllllll}10 & 200 & 190 & 180 & 170 & 160 & 150 & 140 & 130 & 120 & 110 & \begin{array}{c}100 \\ \mathrm{f} 1\end{array}(\mathrm{ppm})\end{array}$ 


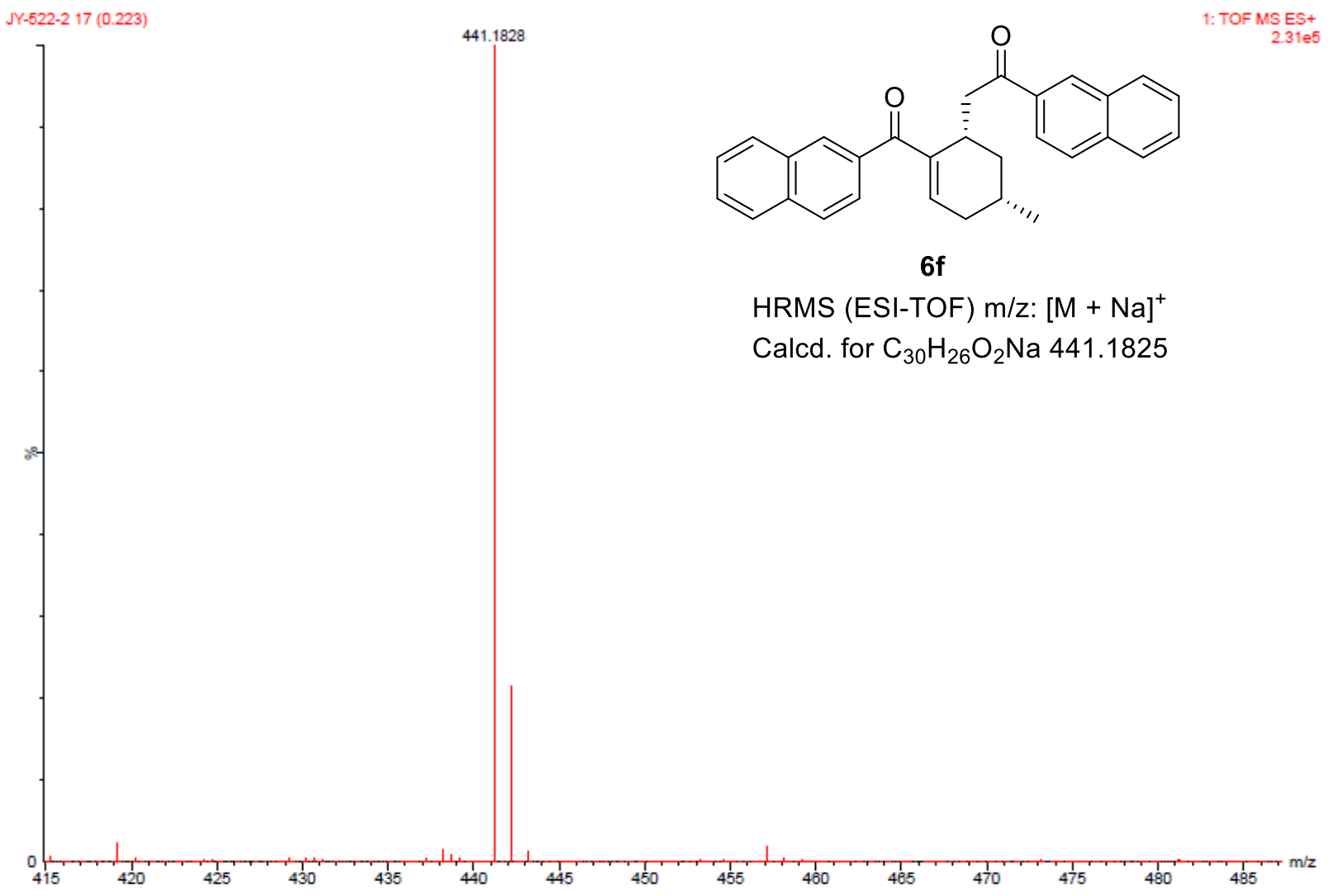




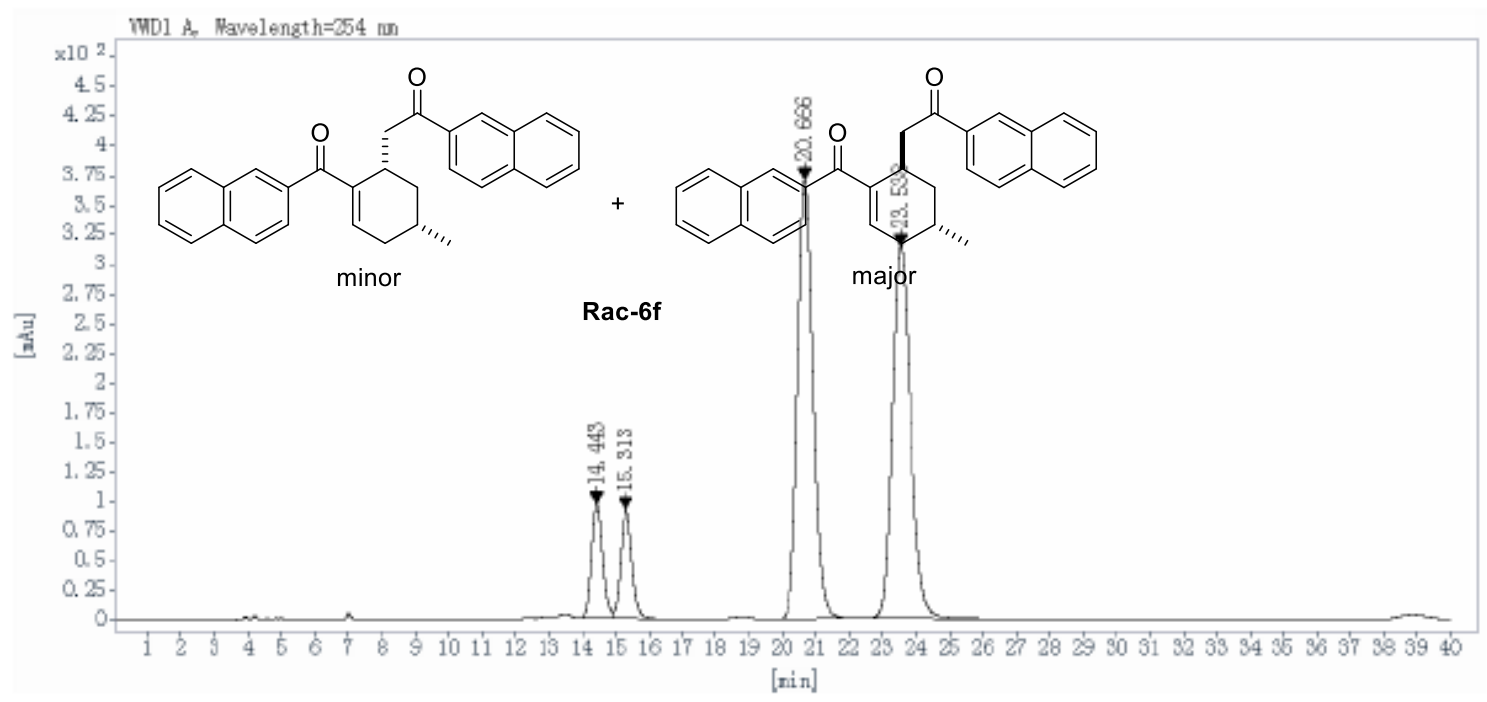

\begin{tabular}{cccccc}
$\begin{array}{c}\text { Ret Time } \\
\text { [min] }\end{array}$ & $\begin{array}{c}\text { Peak } \\
\text { Type }\end{array}$ & $\begin{array}{c}\text { Width } \\
{[\mathrm{min}]}\end{array}$ & $\begin{array}{c}\text { Height } \\
{[\mathrm{mAU}]}\end{array}$ & $\begin{array}{c}\text { Area } \\
{[\mathbf{m A U} * \text { s] }}\end{array}$ & $\begin{array}{c}\text { Area } \\
{[\%]}\end{array}$ \\
\hline 14.443 & BV & 0.35 & 97.0562 & 2168.8181 & 7.7039 \\
15.313 & VB & 0.37 & 92.3319 & 2194.9983 & 7.7969 \\
20.666 & BB & 0.50 & 370.7244 & 11860.6494 & 42.1305 \\
23.538 & BB & 0.59 & 314.5609 & 11927.7031 & 42.3687 \\
& & & Totals: & 28152.1689 & 100.0000
\end{tabular}

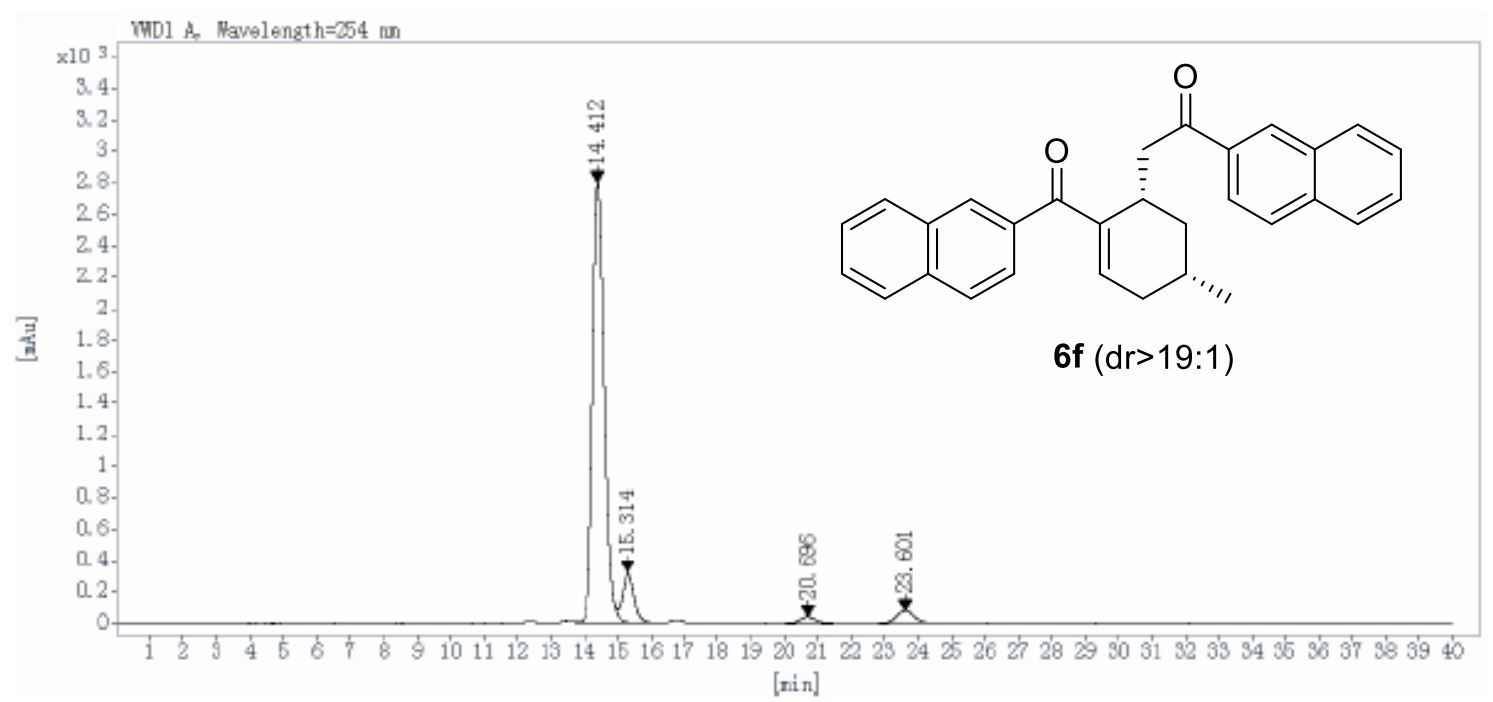

$\begin{array}{cccccc}\begin{array}{c}\text { Ret Time } \\ \text { [min] }\end{array} & \begin{array}{c}\text { Peak } \\ \text { Type }\end{array} & \begin{array}{c}\text { Width } \\ \text { [min] }\end{array} & \begin{array}{c}\text { Height } \\ {[\mathrm{mAU}]}\end{array} & \begin{array}{c}\text { Area } \\ {[\mathrm{mAU} * \text { s] }}\end{array} & \begin{array}{c}\text { Area } \\ {[\%]}\end{array} \\ 14.412 & \text { BV R } & 0.37 & 2792.0183 & 66963.3906 & 84.8917 \\ 15.314 & \text { VB E } & 0.37 & 313.1406 & 7616.4917 & 9.6557 \\ 20.696 & \text { BB } & 0.50 & 39.7952 & 1287.6561 & 1.6324 \\ 23.601 & \text { BB } & 0.58 & 80.2150 & 3013.4182 & 3.8202 \\ & & & \text { Totals: } & 78880.9567 & 100.0000\end{array}$


<smiles>C[C@H]1CC=C(C(=O)c2cccs2)[C@H](CC(=O)c2cccs2)C1</smiles>

$6 \mathrm{~g}$

${ }^{1} \mathrm{H} \mathrm{NMR}\left(400 \mathrm{MHz}, \mathrm{CDCl}_{3}\right)$

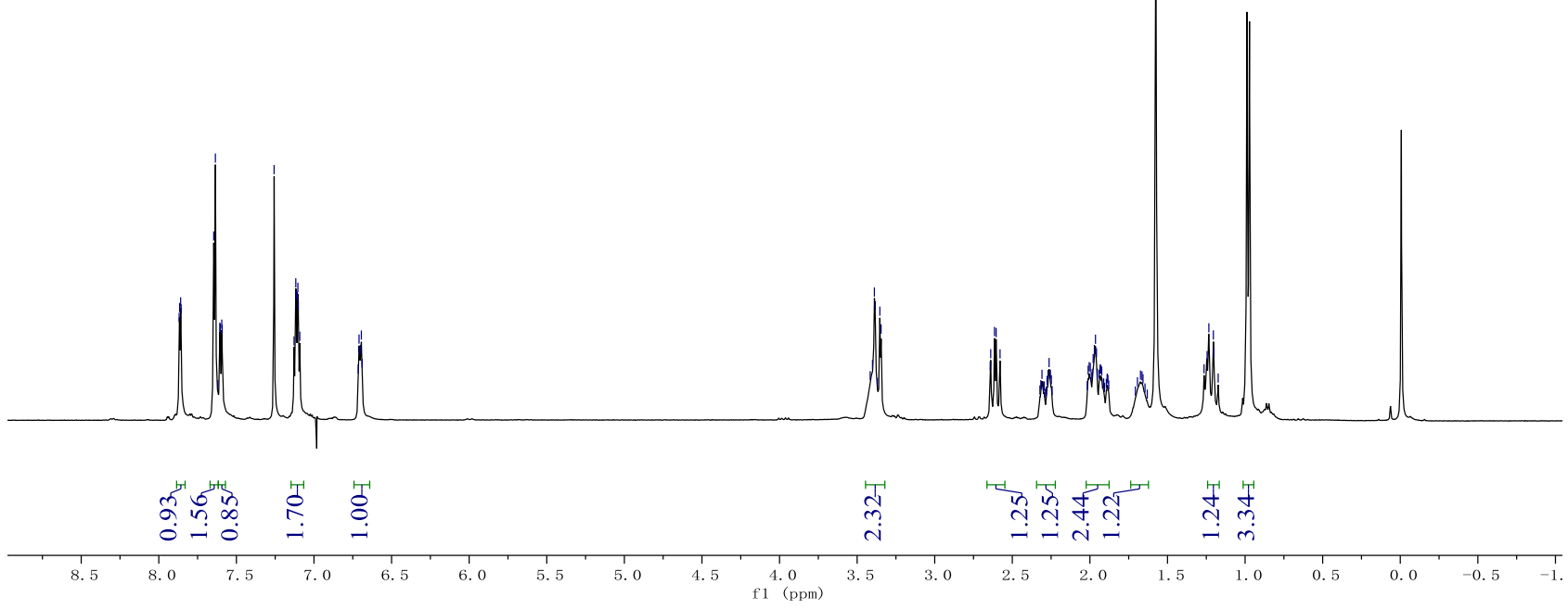

豈点
品

品骂器留嵒嵒品号品器

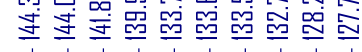<smiles>C[C@H]1CC=C(C(=O)c2cccs2)[C@H](CC(=O)c2cccs2)C1</smiles>

$6 \mathrm{~g}$

${ }^{13} \mathrm{C}$ NMR $\left(100 \mathrm{MHz}, \mathrm{CDCl}_{3}\right)$

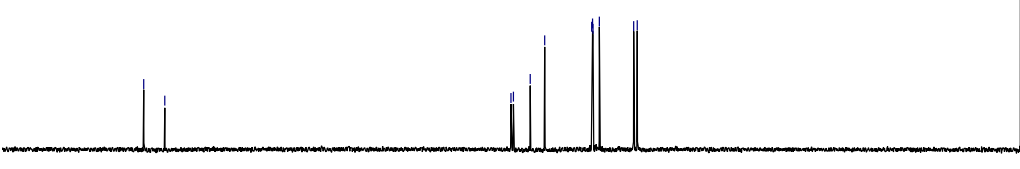

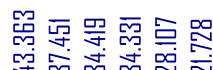

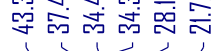




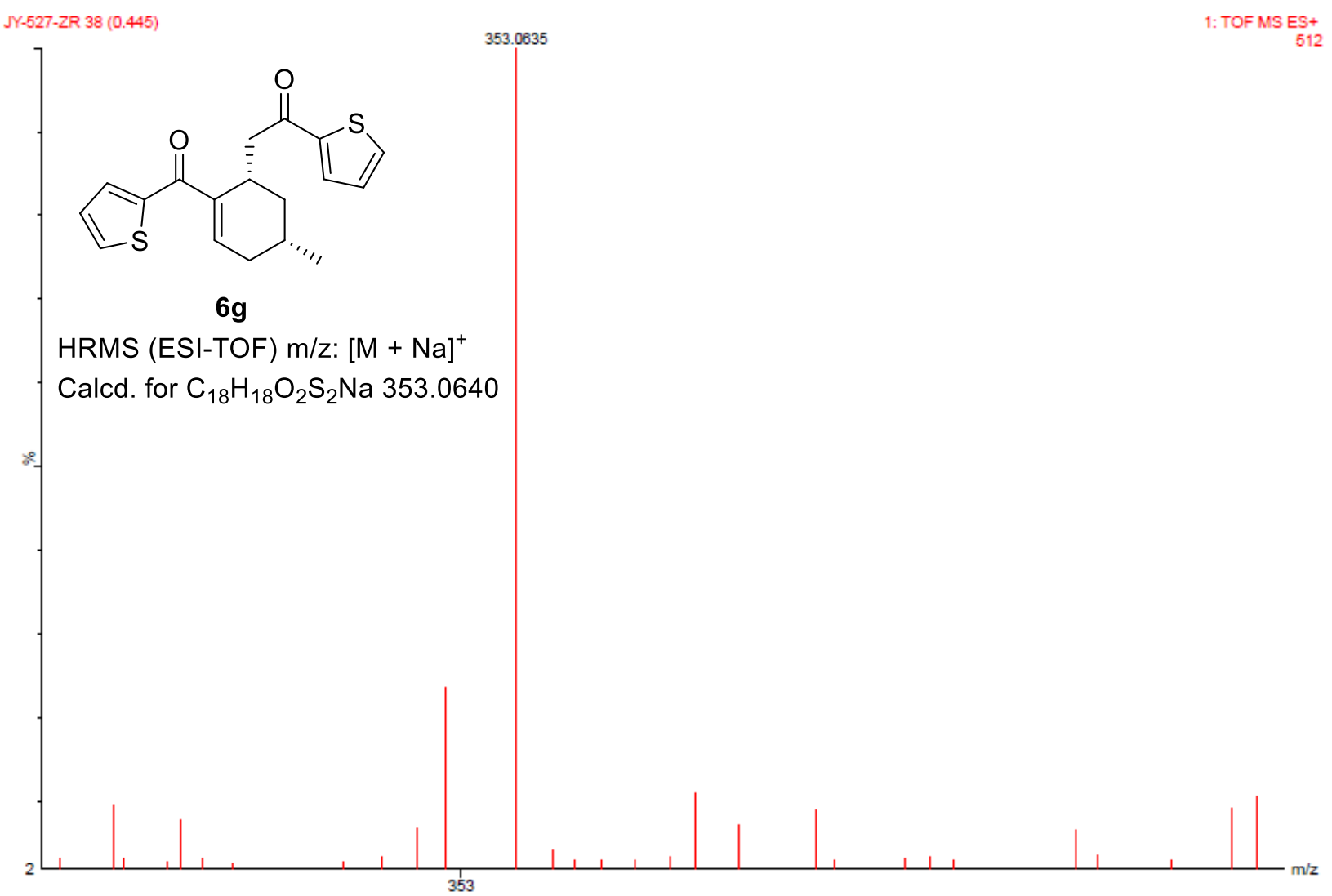




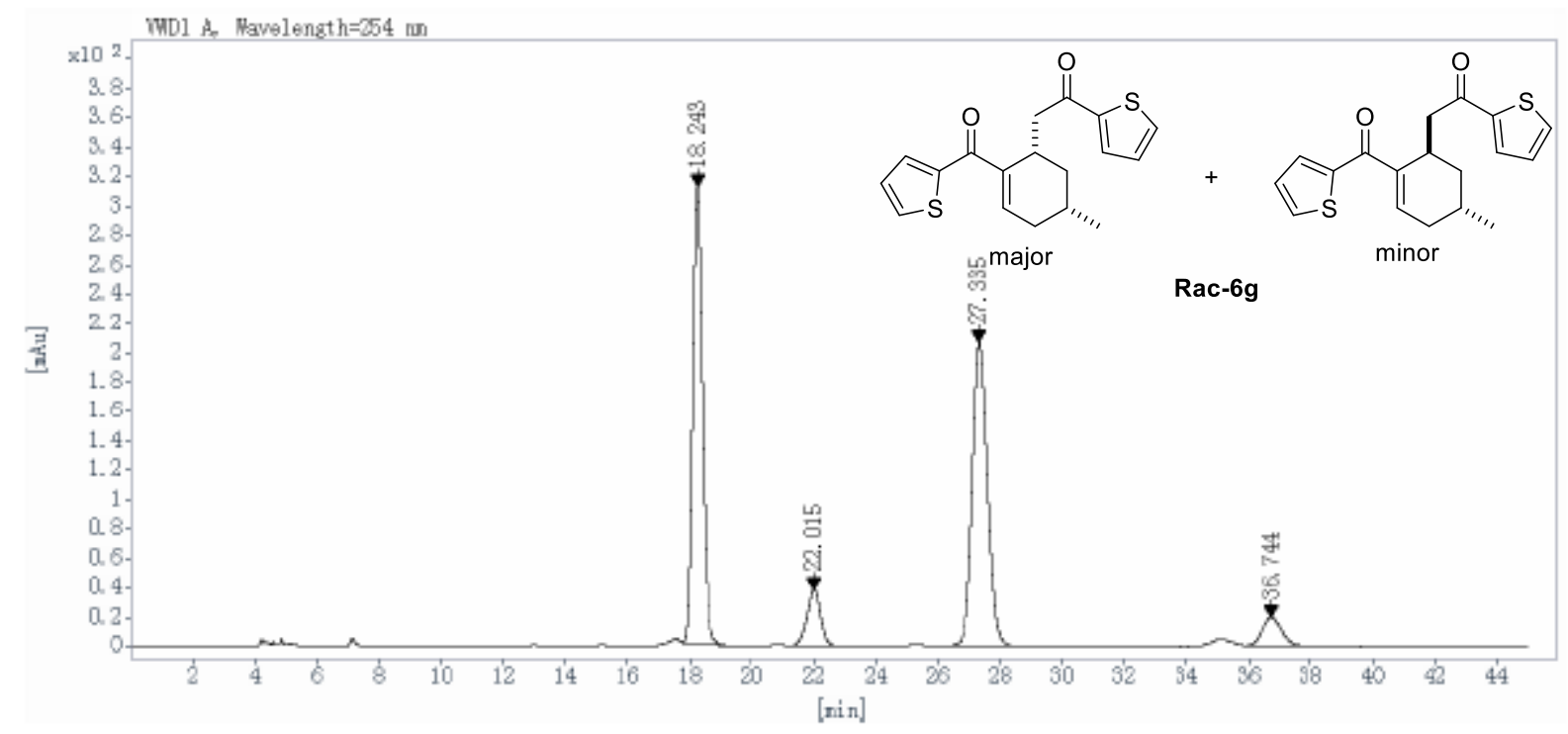

\begin{tabular}{cccccc}
$\begin{array}{c}\text { Ret Time } \\
\text { [min] }\end{array}$ & $\begin{array}{c}\text { Peak } \\
\text { Type }\end{array}$ & $\begin{array}{c}\text { Width } \\
\text { [min] }\end{array}$ & $\begin{array}{c}\text { Height } \\
{[\mathrm{mAU}]}\end{array}$ & $\begin{array}{c}\text { Area } \\
{[\mathrm{mAU} * \text { s] }}\end{array}$ & $\begin{array}{c}\text { Area } \\
{[\%]}\end{array}$ \\
\hline 18.243 & BB & 0.34 & 311.3900 & 6724.5400 & 43.1525 \\
22.015 & BB & 0.45 & 38.6068 & 1144.8969 & 7.3470 \\
27.335 & BBA & 0.52 & 206.2338 & 6875.5356 & 44.1214 \\
36.744 & BB & 0.66 & 19.6501 & 838.2438 & 5.3791 \\
& & & Totals: & 15583.2163 & 100.0000
\end{tabular}

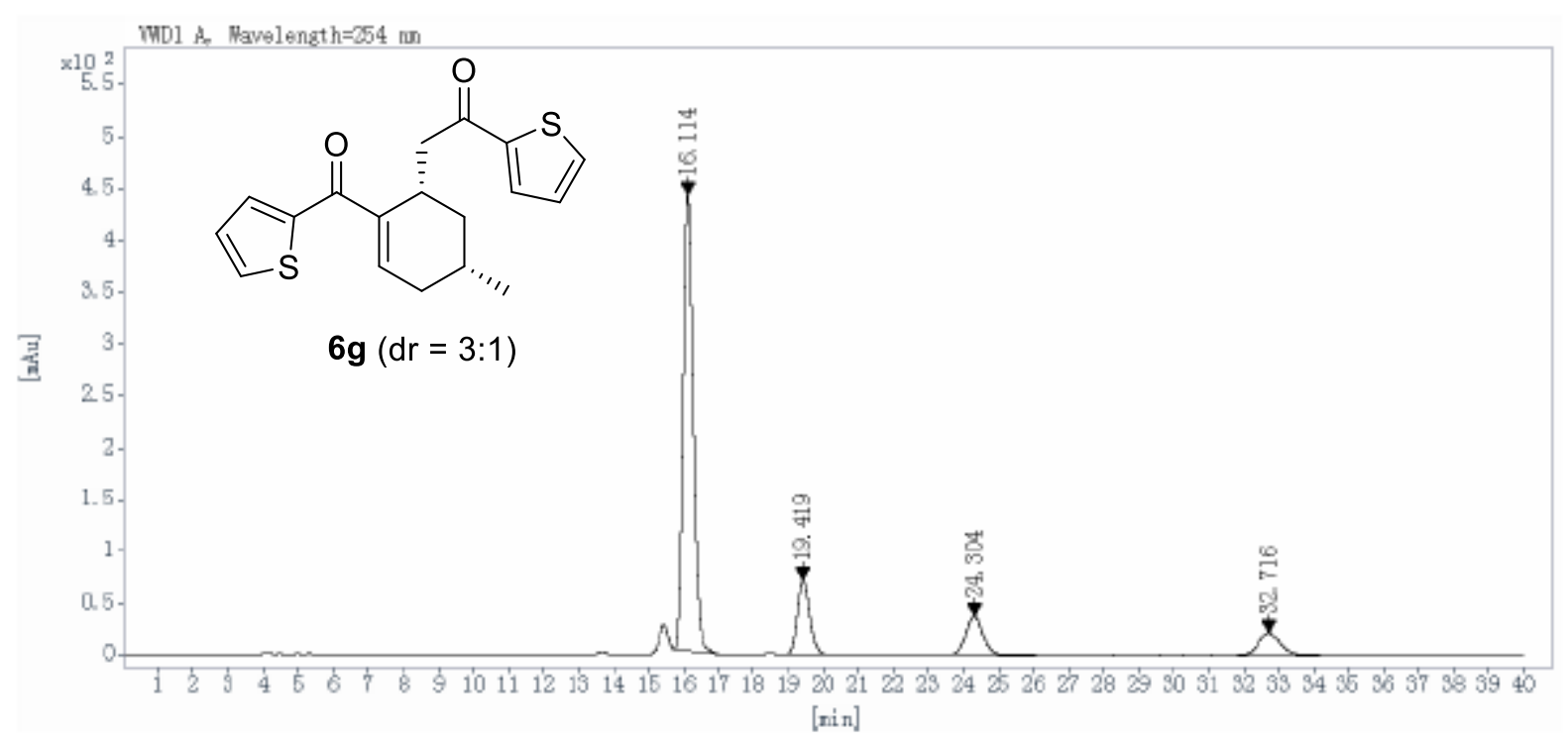

$\begin{array}{cccccc}\begin{array}{c}\text { Ret Time } \\ \text { [min] }\end{array} & \begin{array}{c}\text { Peak } \\ \text { Type }\end{array} & \begin{array}{c}\text { Width } \\ \text { [min] }\end{array} & \begin{array}{c}\text { Height } \\ {[\mathrm{mAU}]}\end{array} & \begin{array}{c}\text { Area } \\ {[\mathrm{mAU} * \text { s] }}\end{array} & \begin{array}{c}\text { Area } \\ {[\%]}\end{array} \\ 16.114 & \text { BB } & 0.30 & 440.0033 & 8593.7461 & 68.7925 \\ 19.419 & \text { BB } & 0.37 & 72.2528 & 1726.1171 & 13.8175 \\ 24.304 & \text { BB } & 0.50 & 37.8416 & 1237.4674 & 9.9059 \\ 32.716 & \text { BB } & 0.65 & 21.9719 & 934.9392 & 7.4841 \\ & & & \text { Totals: } & 12492.2698 & 100.0000\end{array}$


<smiles>O=C(C[C@H]1C[C@H](c2ccccc2)CC=C1C(=O)c1ccccc1)c1ccccc1</smiles>

$6 \mathrm{~h}$

${ }^{1} \mathrm{H}$ NMR $\left(400 \mathrm{MHz}, \mathrm{CDCl}_{3}\right)$

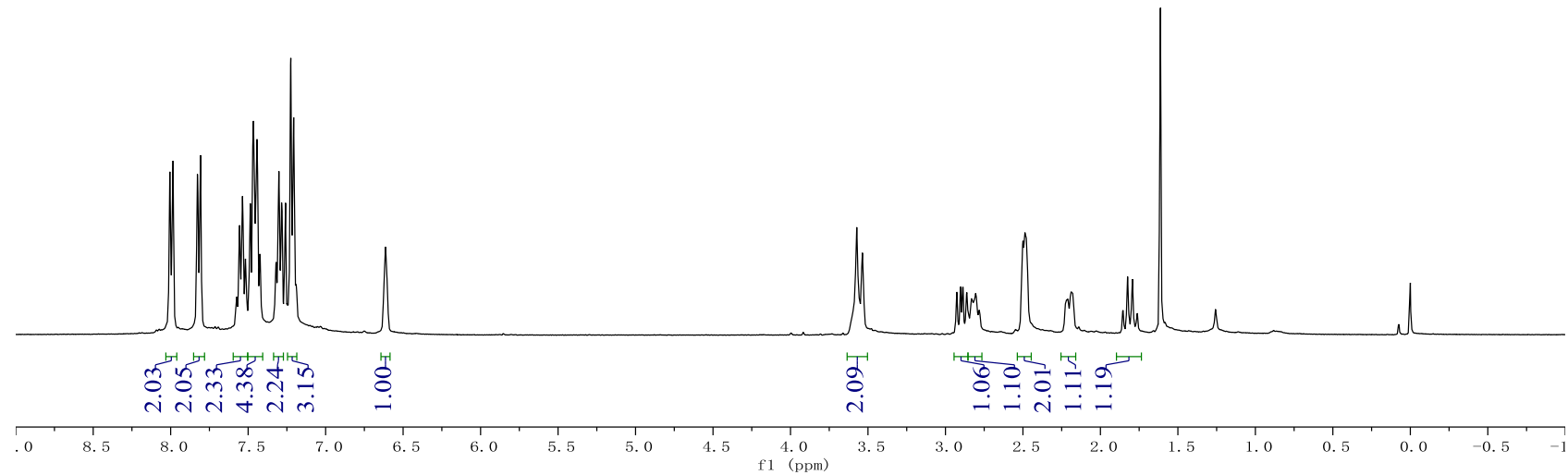

तิ

gे

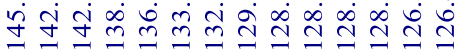<smiles>O=C(C[C@H]1CC(c2ccccc2)CC=C1C(=O)c1ccccc1)c1ccccc1</smiles>

$6 h$

${ }^{13} \mathrm{C}$ NMR $\left(100 \mathrm{MHz}, \mathrm{CDCl}_{3}\right)$

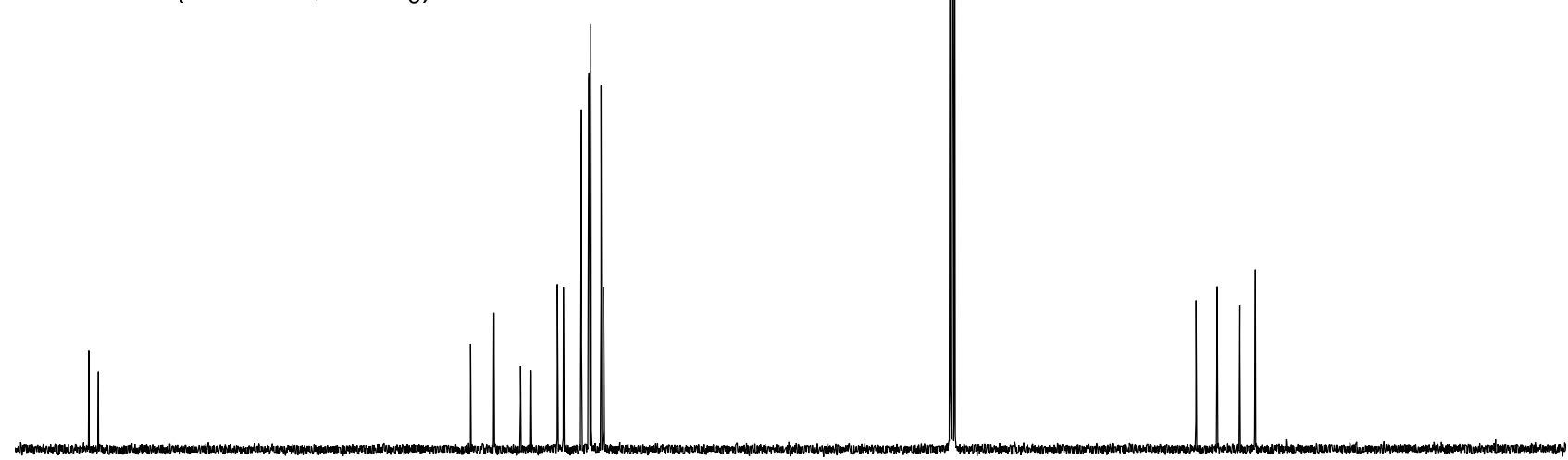




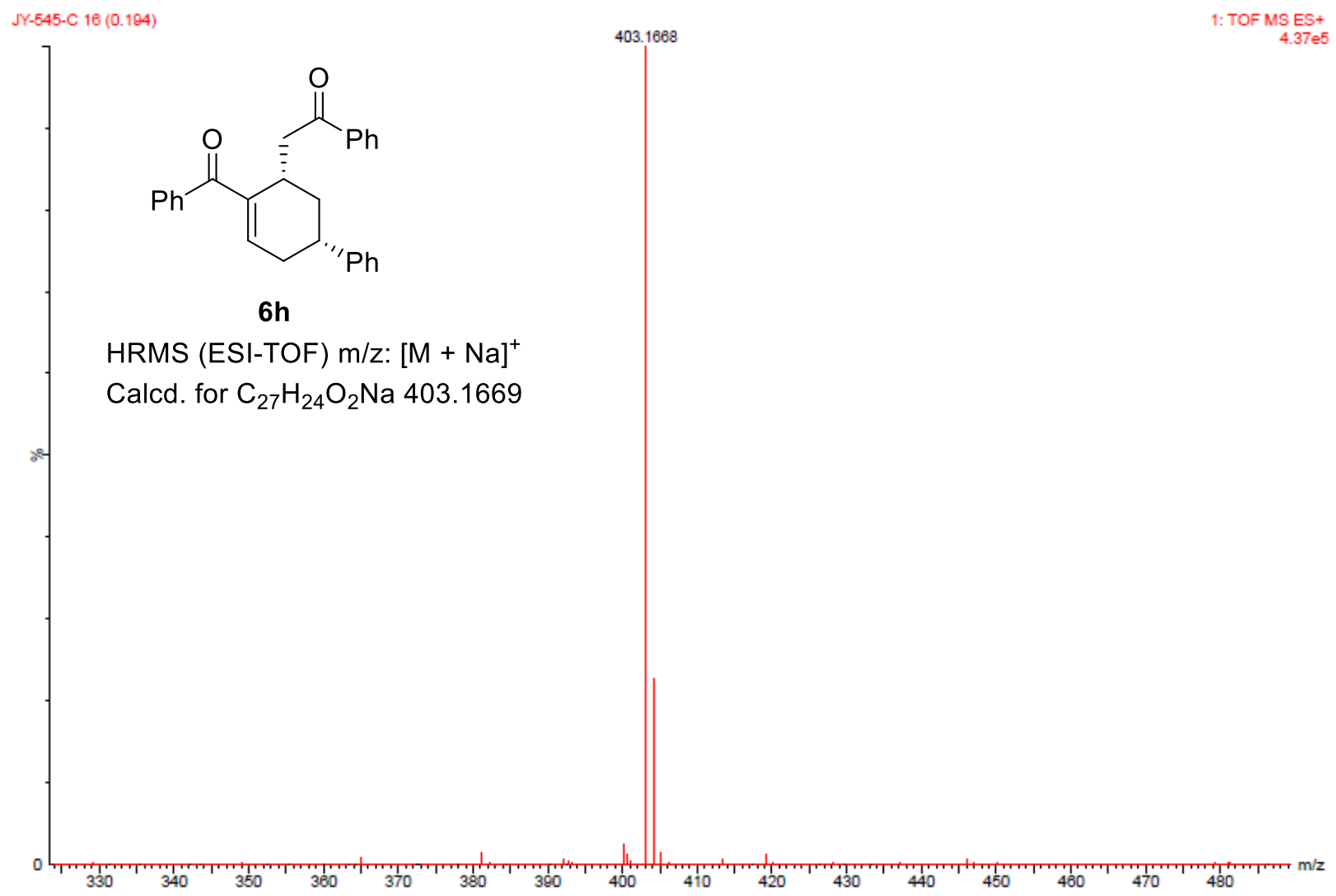




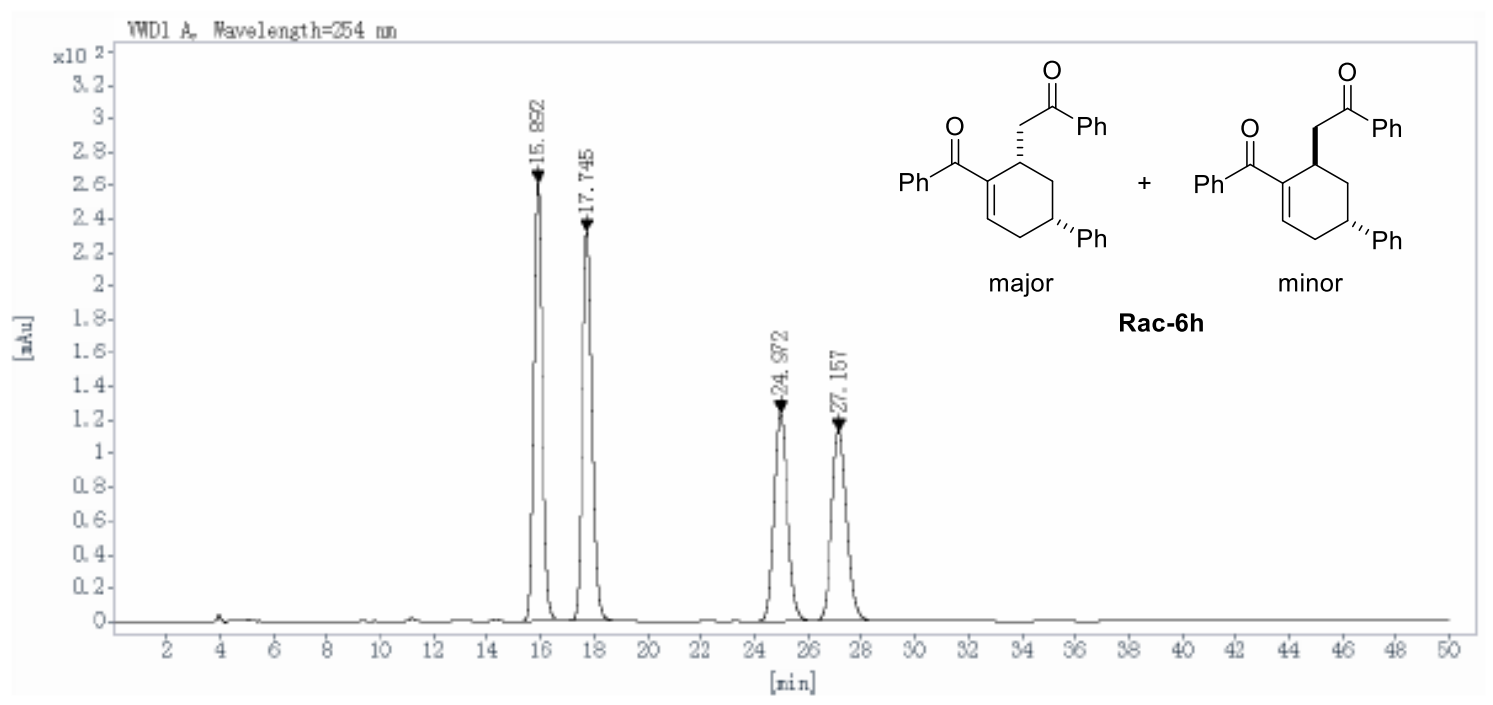

\begin{tabular}{cccccc}
$\begin{array}{c}\text { Ret Time } \\
\text { [min] }\end{array}$ & $\begin{array}{c}\text { Peak } \\
\text { Type }\end{array}$ & $\begin{array}{c}\text { Width } \\
{[\mathrm{min}]}\end{array}$ & $\begin{array}{c}\text { Height } \\
{[\mathrm{mAU}]}\end{array}$ & $\begin{array}{c}\text { Area } \\
{[\mathrm{mAU} \text { *s] }}\end{array}$ & $\begin{array}{c}\text { Area } \\
{[\%]}\end{array}$ \\
\hline 15.892 & BB & 0.35 & 261.3147 & 5905.1514 & 28.5965 \\
17.745 & BB & 0.40 & 232.4285 & 5921.3423 & 28.6749 \\
24.972 & BB & 0.55 & 124.6440 & 4407.6489 & 21.3446 \\
27.157 & BB & 0.60 & 113.7442 & 4415.7637 & 21.3839 \\
& & & Totals: & 20649.9063 & 100.0000
\end{tabular}

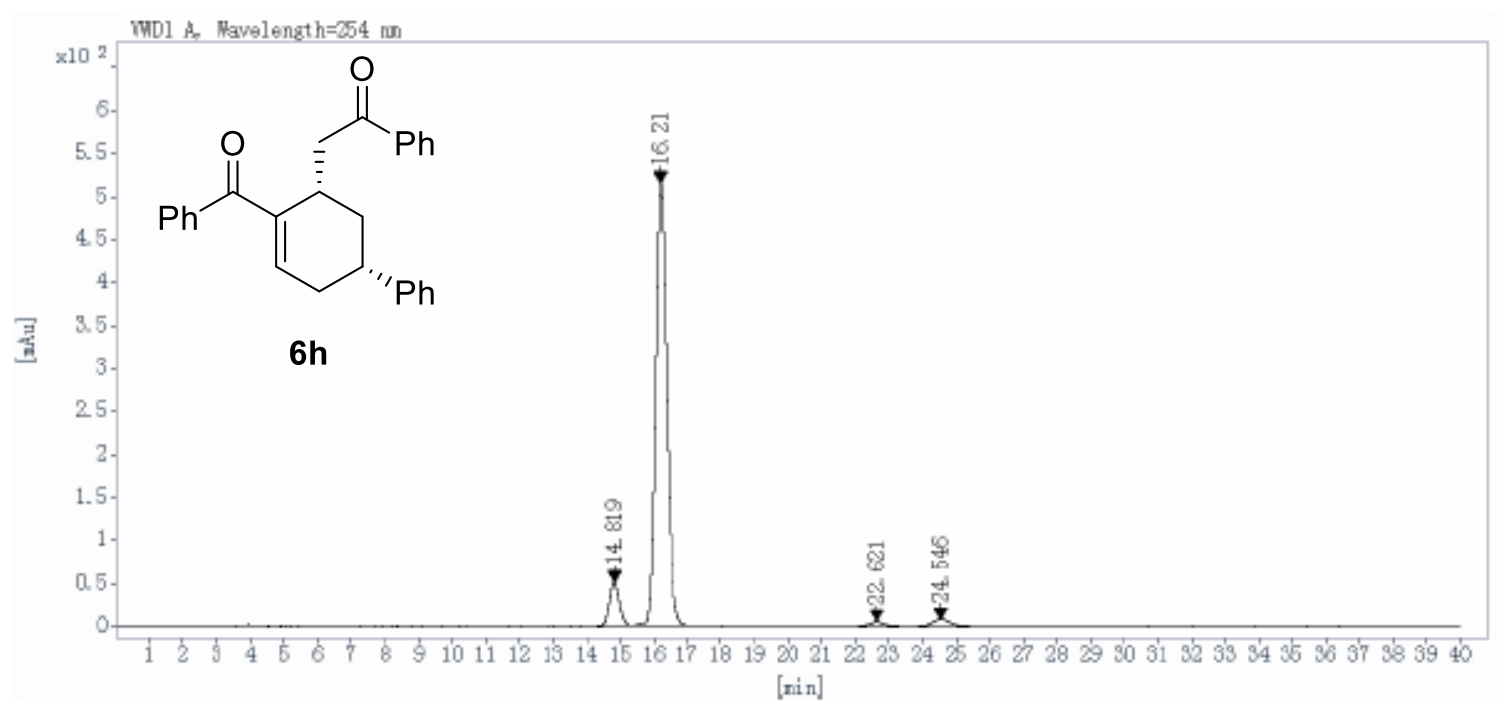

$\begin{array}{cccccc}\begin{array}{c}\text { Ret Time } \\ \text { [min] }\end{array} & \begin{array}{c}\text { Peak } \\ \text { Type }\end{array} & \begin{array}{c}\text { Width } \\ {[\mathrm{min}]}\end{array} & \begin{array}{c}\text { Height } \\ {[\mathrm{mAU}]}\end{array} & \begin{array}{c}\text { Area } \\ {\left[\mathrm{mAU}^{*} \text { s] }\right.}\end{array} & \begin{array}{c}\text { Area } \\ {[\%]}\end{array} \\ 14.819 & \text { BV } & 0.31 & 50.5505 & 1020.7421 & 7.7468 \\ 16.210 & \text { VB } & 0.35 & 515.2905 & 11717.4092 & 88.9280 \\ 22.621 & \text { BB } & 0.48 & 4.9890 & 154.7931 & 1.1748 \\ 24.546 & \text { BB } & 0.52 & 8.3051 & 283.3459 & 2.1504 \\ & & & \text { Totals: } & 13176.2903 & 100.0000\end{array}$


<smiles>O=C(C[C@H]1C[C@H](c2ccc(Cl)cc2)CC=C1C(=O)c1ccccc1)c1ccccc1</smiles>

$6 \mathbf{i}$

${ }^{1} \mathrm{H}$ NMR $\left(400 \mathrm{MHz}, \mathrm{CDCl}_{3}\right)$

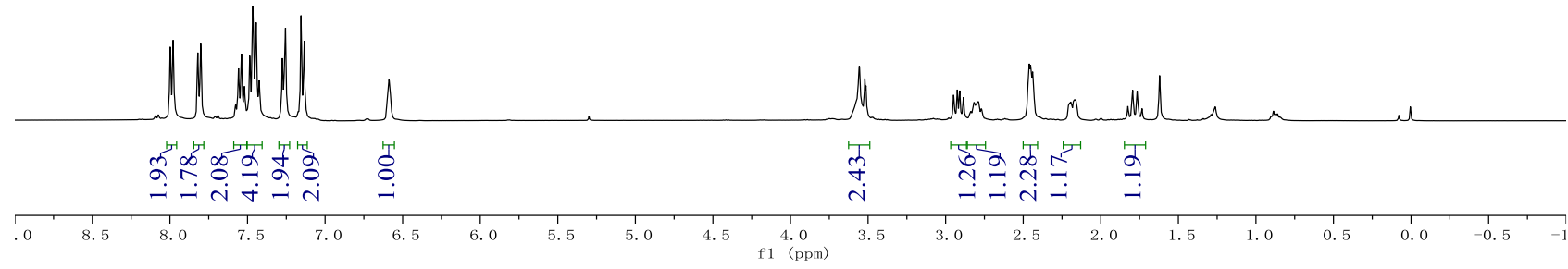

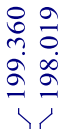

तิ

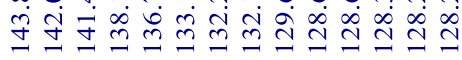

mై

भे<smiles>O=C(C[C@H]1C[C@H](c2ccc(Cl)cc2)CC=C1C(=O)c1ccccc1)c1ccccc1</smiles>

$6 \mathbf{i}$

${ }^{13} \mathrm{C}$ NMR $\left(100 \mathrm{MHz}, \mathrm{CDCl}_{3}\right)$

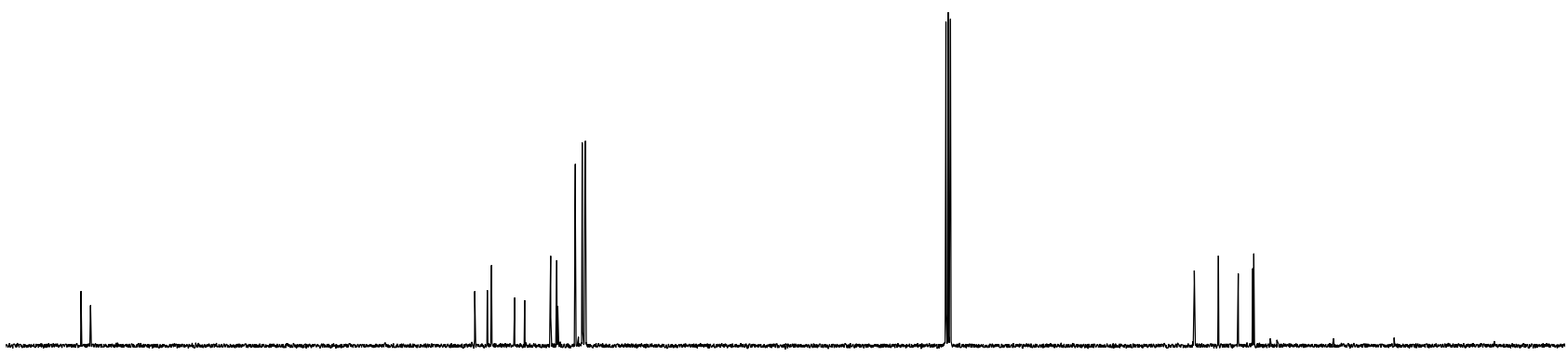



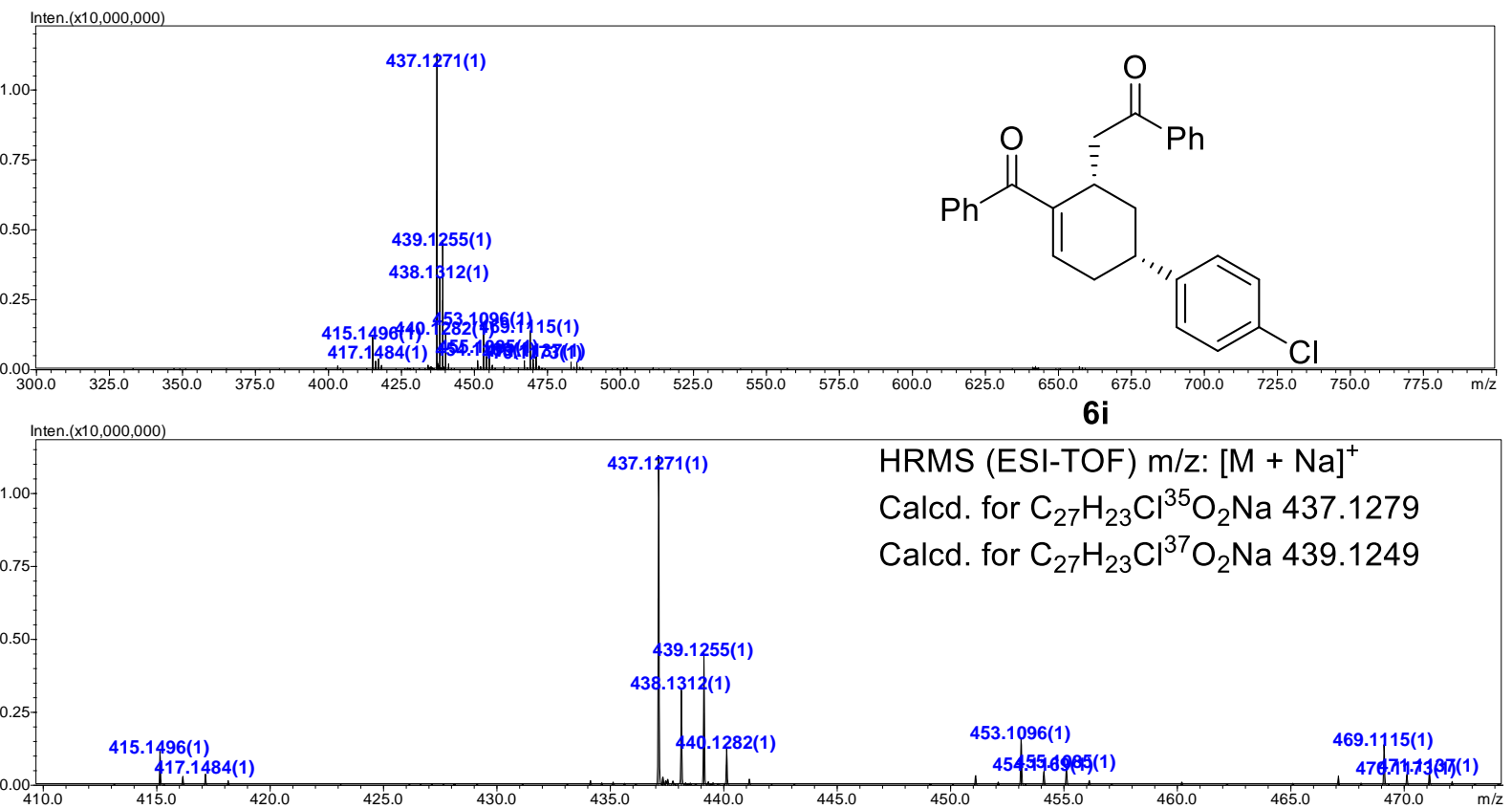
MDI A. Navelength=254 in

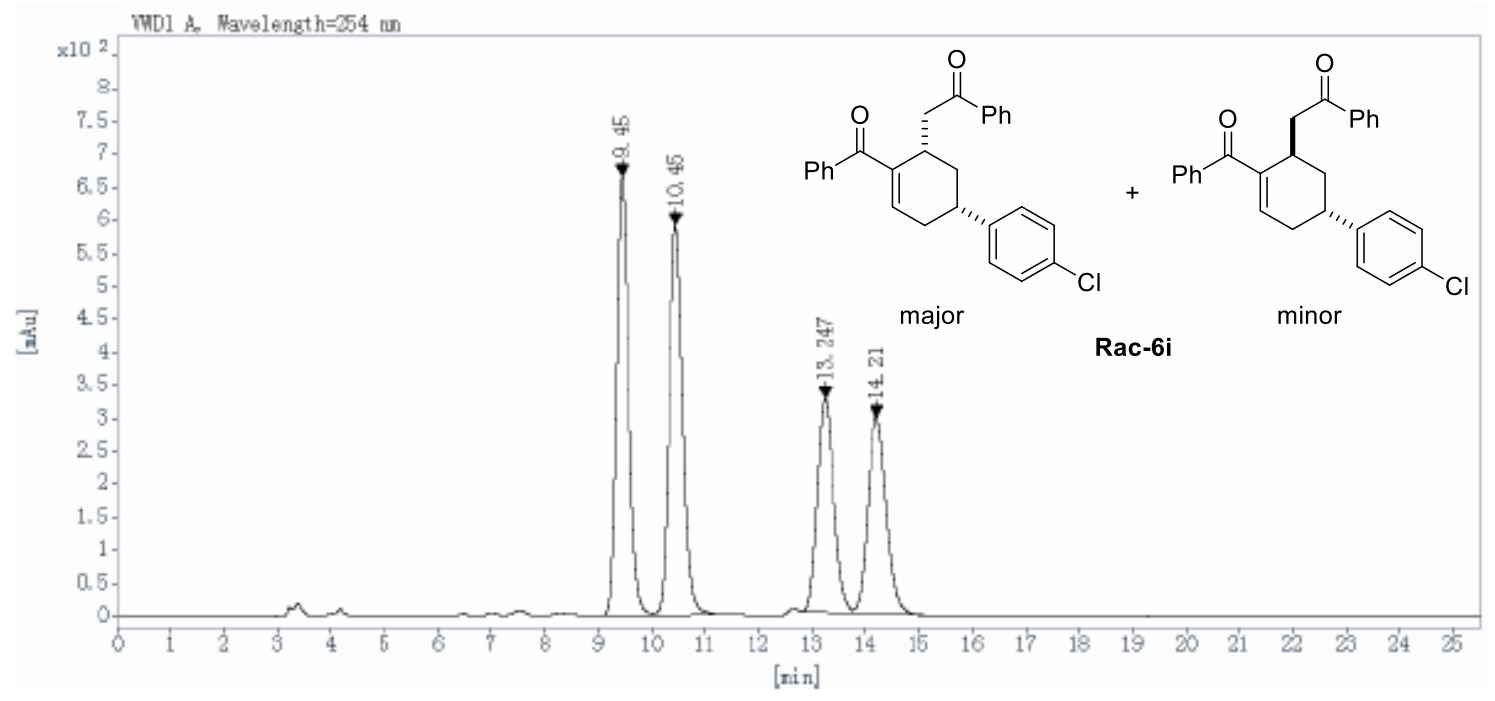

$\begin{array}{cccccc}\begin{array}{c}\text { Ret Time } \\ \text { [min] }\end{array} & \begin{array}{c}\text { Peak } \\ \text { Type }\end{array} & \begin{array}{c}\text { Width } \\ \text { [min] }\end{array} & \begin{array}{c}\text { Height } \\ \text { [mAU] }\end{array} & \begin{array}{c}\text { Area } \\ {\left[\mathrm{mAU} *_{\text {s }}\right]}\end{array} & \begin{array}{c}\text { Area } \\ {[\%]}\end{array} \\ 9.450 & \text { BV } & 0.24 & 666.3967 & 10558.4014 & 29.9803 \\ 10.450 & \text { VB } & 0.27 & 591.4036 & 10440.0273 & 29.6442 \\ 13.247 & \text { BV } & 0.34 & 324.6529 & 7051.0684 & 20.0213 \\ 14.210 & \text { VB } & 0.37 & 299.1977 & 7168.2734 & 20.3541 \\ & & & \text { Totals: } & 35217.7705 & 100.0000\end{array}$

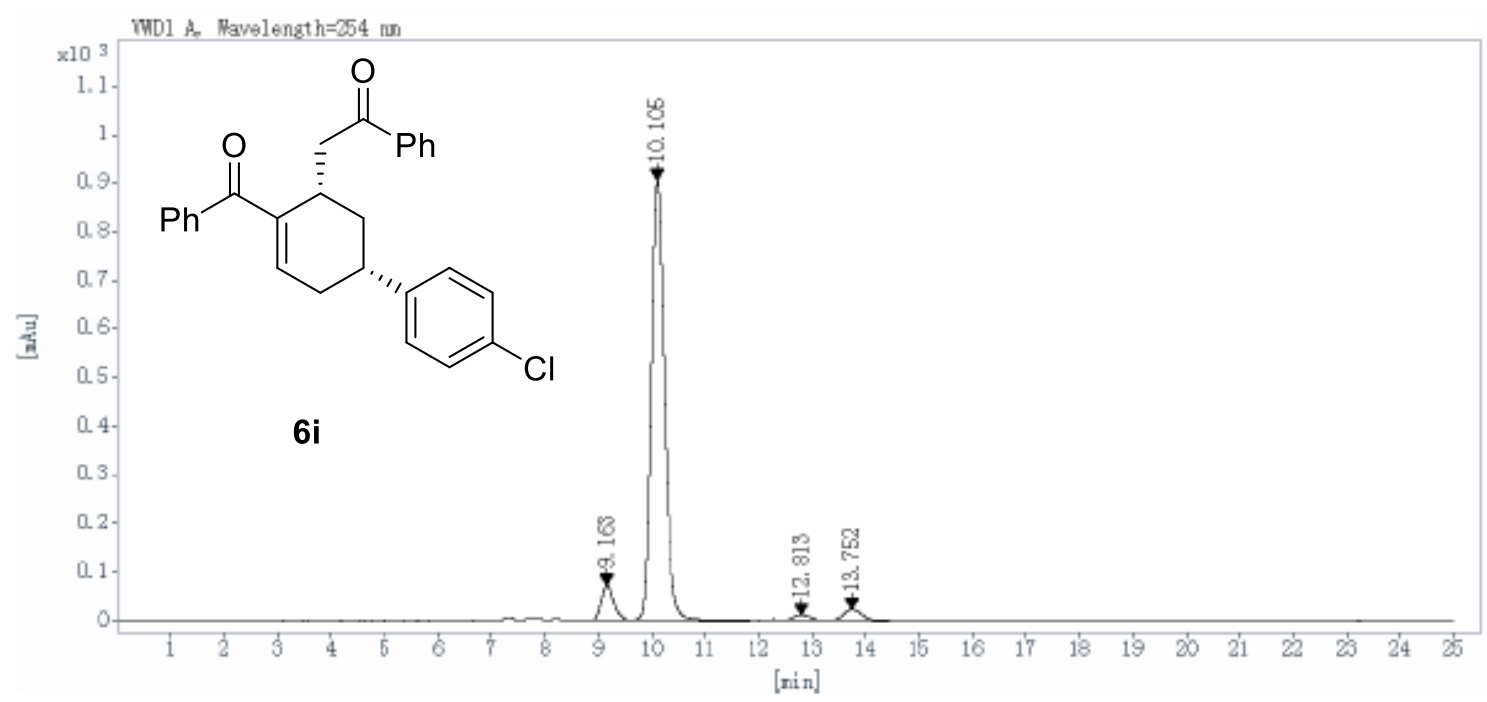

$\begin{array}{cccccc}\begin{array}{c}\text { Ret Time } \\ \text { [min] }\end{array} & \begin{array}{c}\text { Peak } \\ \text { Type }\end{array} & \begin{array}{c}\text { Width } \\ \text { [min] }\end{array} & \begin{array}{c}\text { Height } \\ \text { [mAU] }\end{array} & \begin{array}{c}\text { Area } \\ {\left[\mathrm{mAU} *_{\text {s] }}\right.}\end{array} & \begin{array}{c}\text { Area } \\ {[\%]}\end{array} \\ 9.163 & \text { BV } & 0.24 & 73.4739 & 1171.9808 & 6.7613 \\ 10.105 & \text { VB } & 0.26 & 904.4433 & 15406.2949 & 88.8814 \\ 12.813 & \text { BB } & 0.32 & 11.2489 & 226.4591 & 1.3065 \\ 13.752 & \text { BB } & 0.36 & 22.8725 & 528.8088 & 3.0508 \\ & & & \text { Totals: } & 17333.5437 & 100.0000\end{array}$




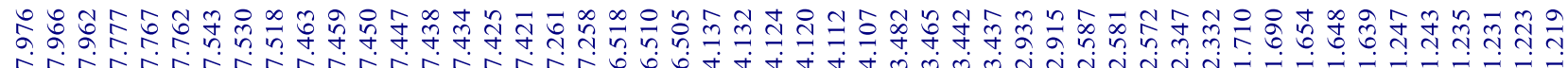<smiles>CCOC(=O)[C@H]1CC=C(C(=O)c2ccccc2)[C@H](CC(=O)c2ccccc2)C1</smiles>

$6 \mathbf{j}$

${ }^{1} \mathrm{H} \mathrm{NMR}\left(600 \mathrm{MHz}, \mathrm{CDCl}_{3}\right)$

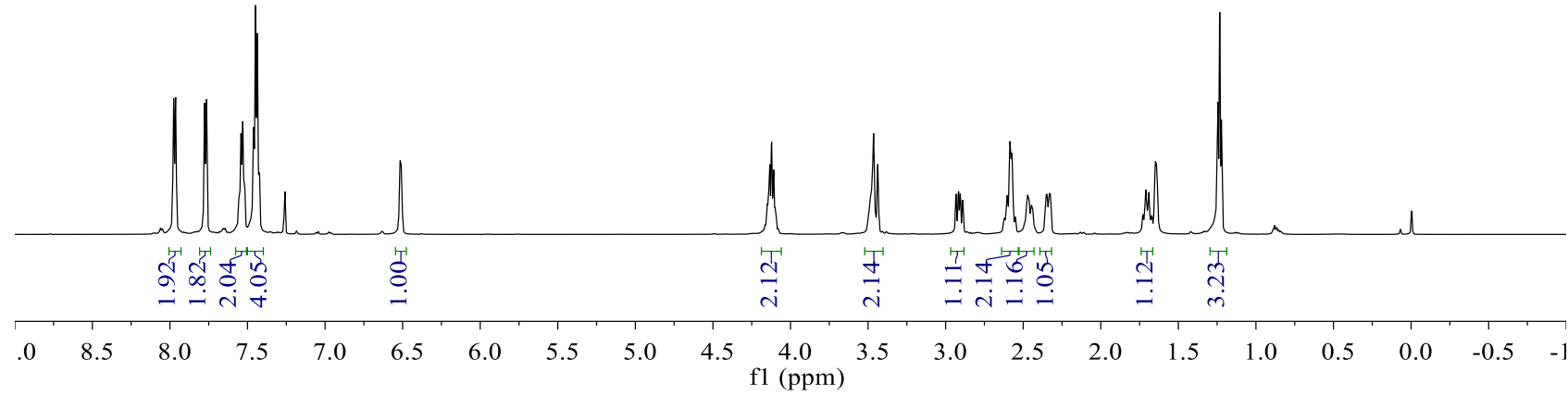<smiles>CCOC(=O)[C@H]1CC=C(C(=O)c2ccccc2)[C@H](CC(=O)c2ccccc2)C1</smiles>

6j

${ }^{13} \mathrm{C} \mathrm{NMR}\left(150 \mathrm{MHz}, \mathrm{CDCl}_{3}\right)$

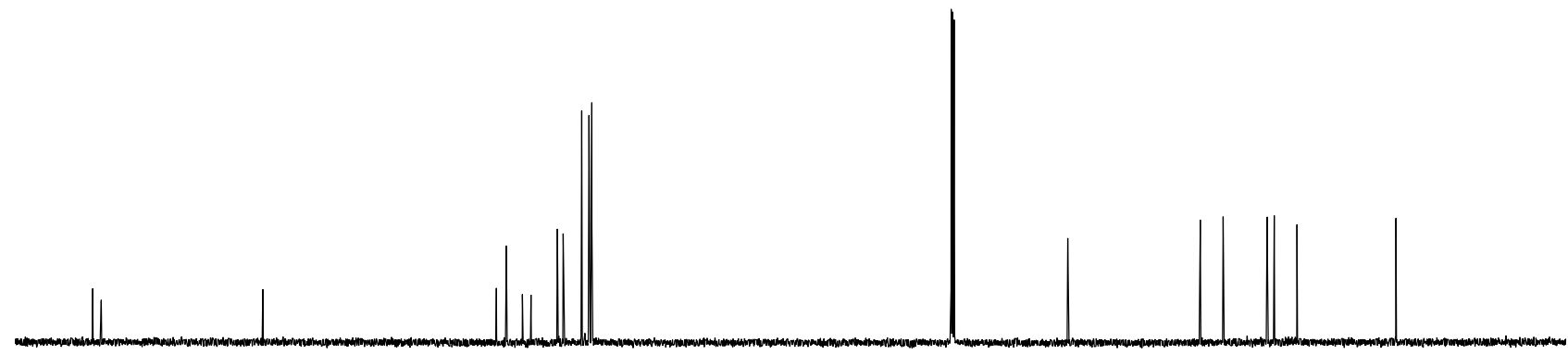

$\begin{array}{llllllllllllllllllllll}200 & 190 & 180 & 170 & 160 & 150 & 140 & 130 & 120 & 110 \begin{array}{c}100 \\ \mathrm{f} 1(\mathrm{ppm})\end{array} & 90 & 80 & 70 & 60 & 50 & 40 & 30 & 20 & 10 & 0 & -\end{array}$ 


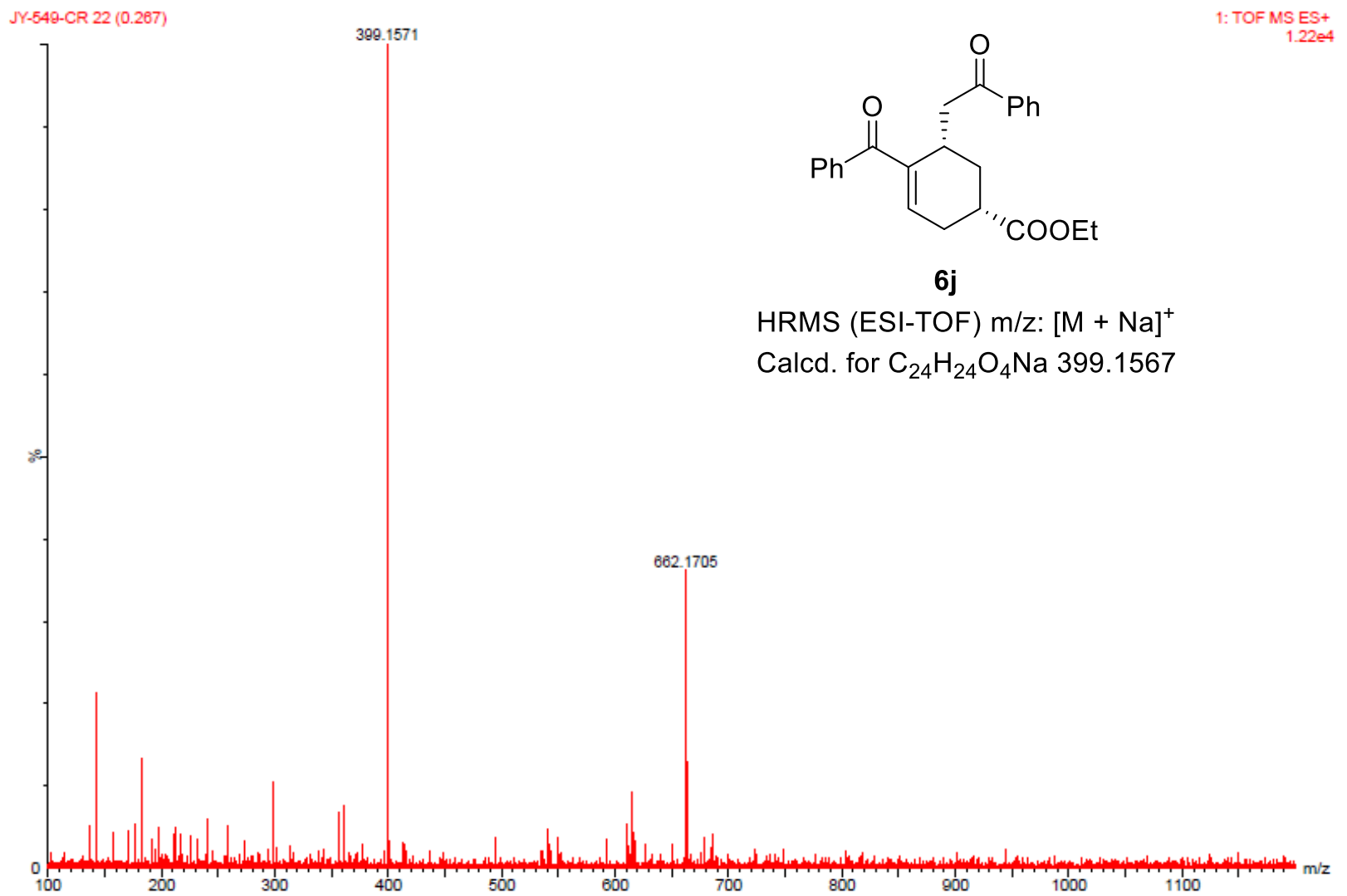


wD1 A Havelength $=254$ III

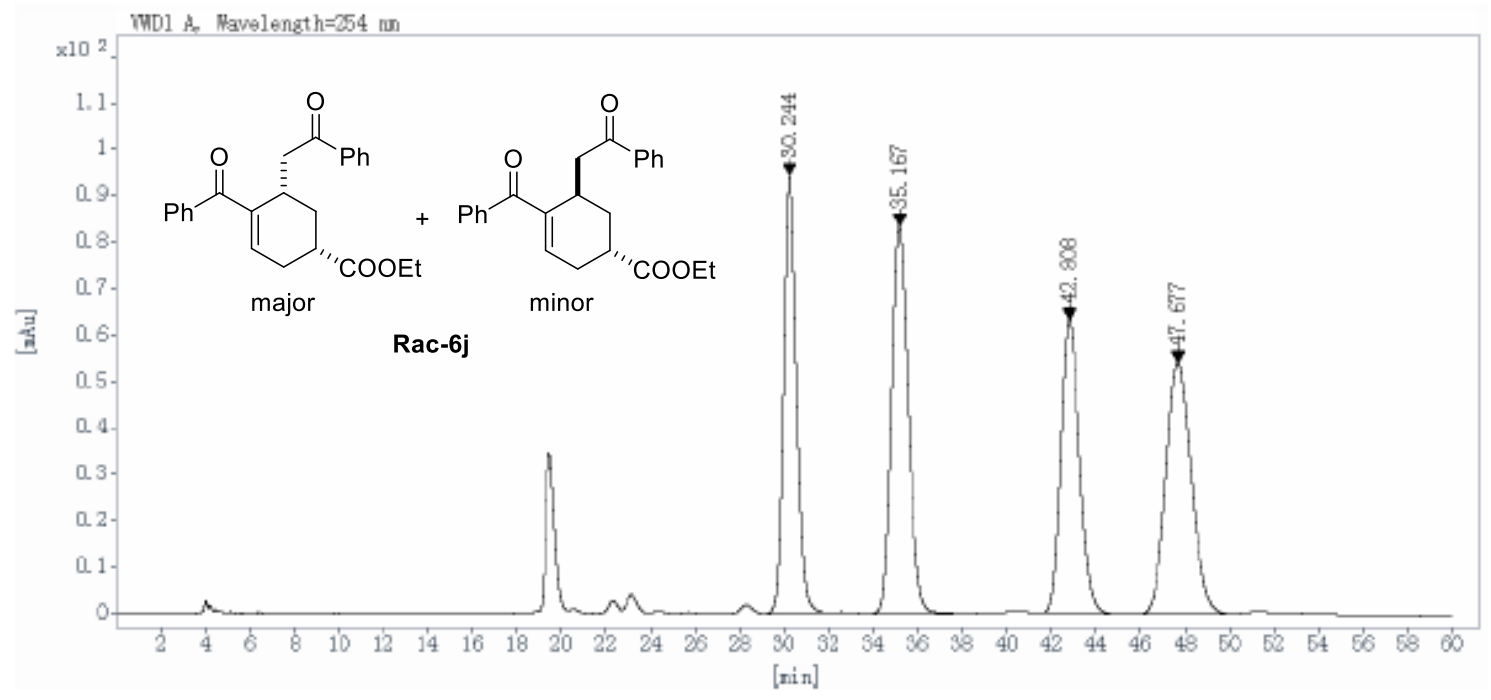

$\begin{array}{cccccc}\begin{array}{c}\text { Ret Time } \\ \text { [min] }\end{array} & \begin{array}{c}\text { Peak } \\ \text { Type }\end{array} & \begin{array}{c}\text { Width } \\ \text { [min] }\end{array} & \begin{array}{c}\text { Height } \\ \text { [mAU] }\end{array} & \begin{array}{c}\text { Area } \\ \text { [mAU*'s] }\end{array} & \begin{array}{c}\text { Area } \\ {[\%]}\end{array} \\ 30.244 & \text { BB } & 0.63 & 94.3248 & 3875.2522 & 23.9167 \\ 35.167 & \text { BB } & 0.80 & 83.5544 & 4339.4180 & 26.7813 \\ 42.808 & \text { BB } & 0.89 & 63.3727 & 3696.8967 & 22.8159 \\ 47.677 & \text { BB } & 1.20 & 54.1909 & 4291.5679 & 26.4860 \\ & & & \text { Totals: } & 16203.1348 & 100.0000\end{array}$

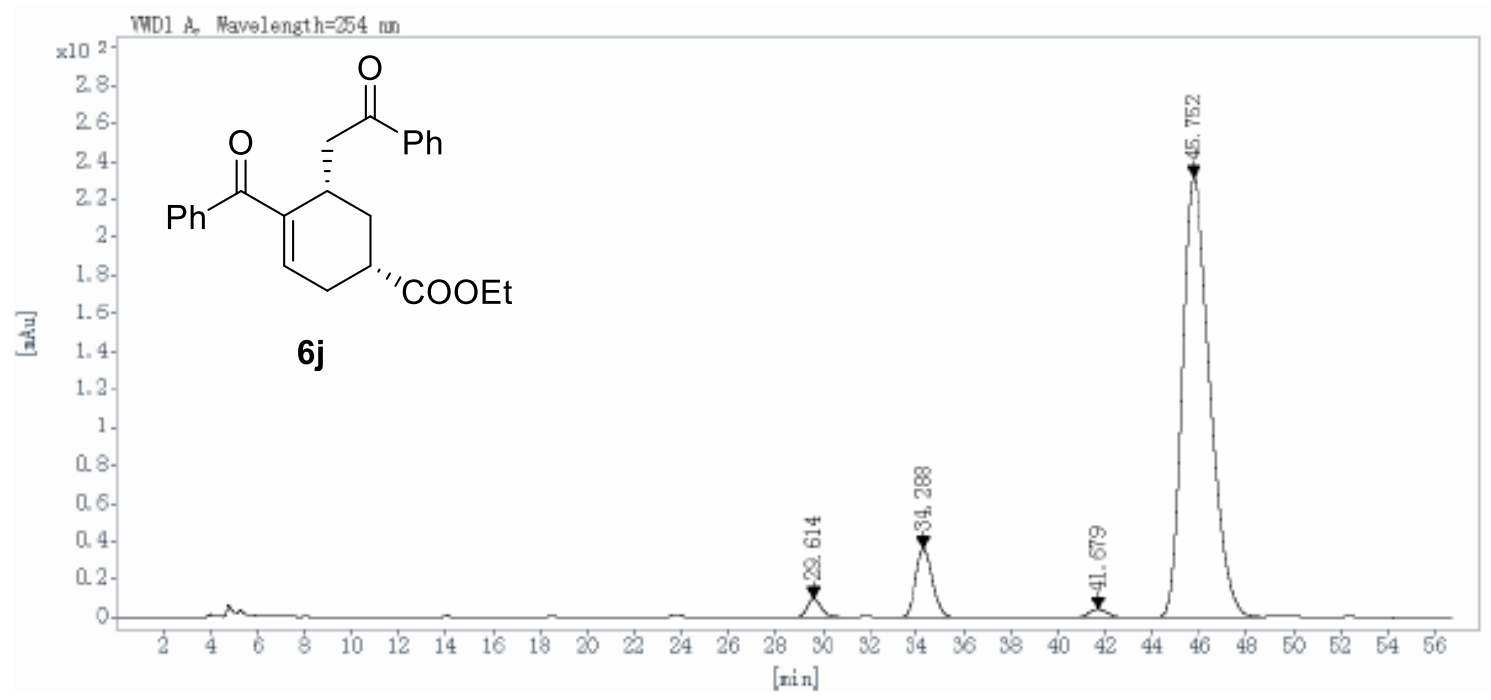

\begin{tabular}{cccccc}
$\begin{array}{c}\text { Ret Time } \\
\text { [min] }\end{array}$ & $\begin{array}{c}\text { Peak } \\
\text { Type }\end{array}$ & $\begin{array}{c}\text { Width } \\
\text { [min] }\end{array}$ & $\begin{array}{c}\text { Height } \\
\text { [mAU] }\end{array}$ & $\begin{array}{c}\text { Area } \\
{\left[\mathrm{mAU} *_{\text {s] }}\right.}\end{array}$ & $\begin{array}{c}\text { Area } \\
{[\%]}\end{array}$ \\
\hline 29.614 & BB & 0.62 & 10.0783 & 414.3276 & 1.9538 \\
34.288 & BB & 0.77 & 36.5745 & 1812.5671 & 8.5472 \\
41.679 & BB & 0.78 & 4.4221 & 245.2777 & 1.1566 \\
45.752 & BB & 1.22 & 231.4505 & 18734.2871 & 88.3424 \\
& & & Totals: & 21206.4596 & 100.0000
\end{tabular}


<smiles>CCCCO[C@H]1CC=C(C(=O)c2ccccc2)[C@H](CC(=O)c2ccccc2)C1</smiles>

6k $(\mathrm{dr}=10: 1)$

${ }^{1} \mathrm{H}$ NMR $\left(400 \mathrm{MHz}, \mathrm{CDCl}_{3}\right)$

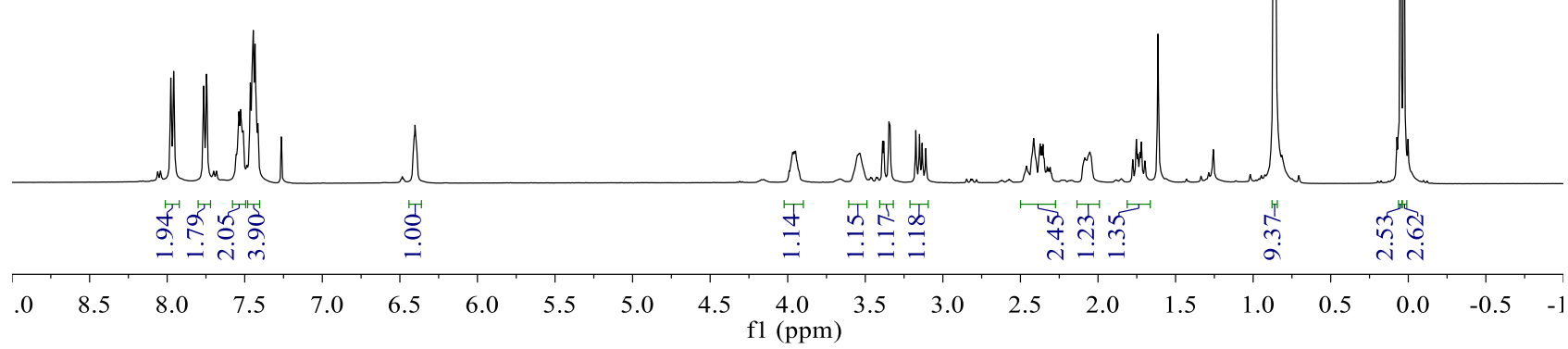

‡i

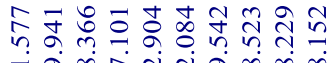

完

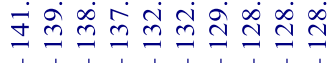

midi

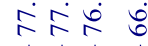

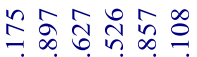

नुलिलिं

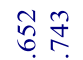

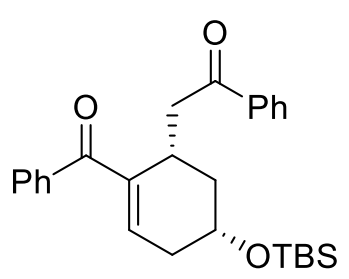

6k $(\mathrm{dr}=10: 1)$

${ }^{13} \mathrm{C}$ NMR (100 MHz, $\left.\mathrm{CDCl}_{3}\right)$

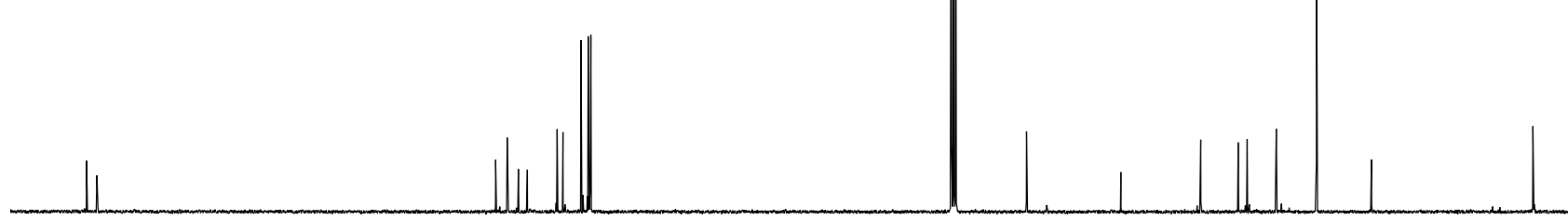

$\begin{array}{lllllllllllllllllllllll}10 & 200 & 190 & 180 & 170 & 160 & 150 & 140 & 130 & 120 & 110 & \begin{array}{c}100 \\ \mathrm{f} 1\end{array}(\mathrm{ppm}) & 90 & 80 & 70 & 60 & 50 & 40 & 30 & 20 & 10 & 0 & -\end{array}$ 


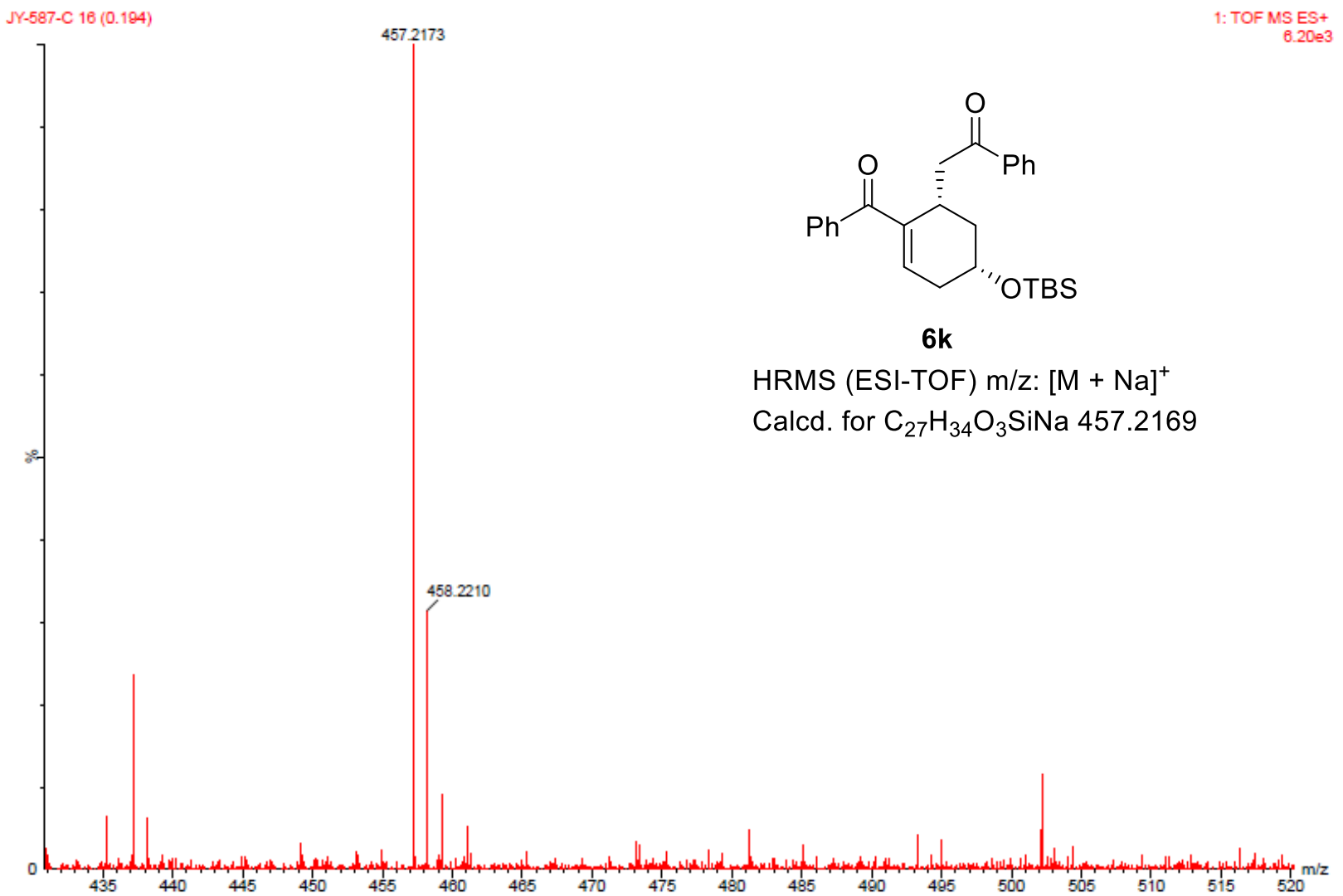




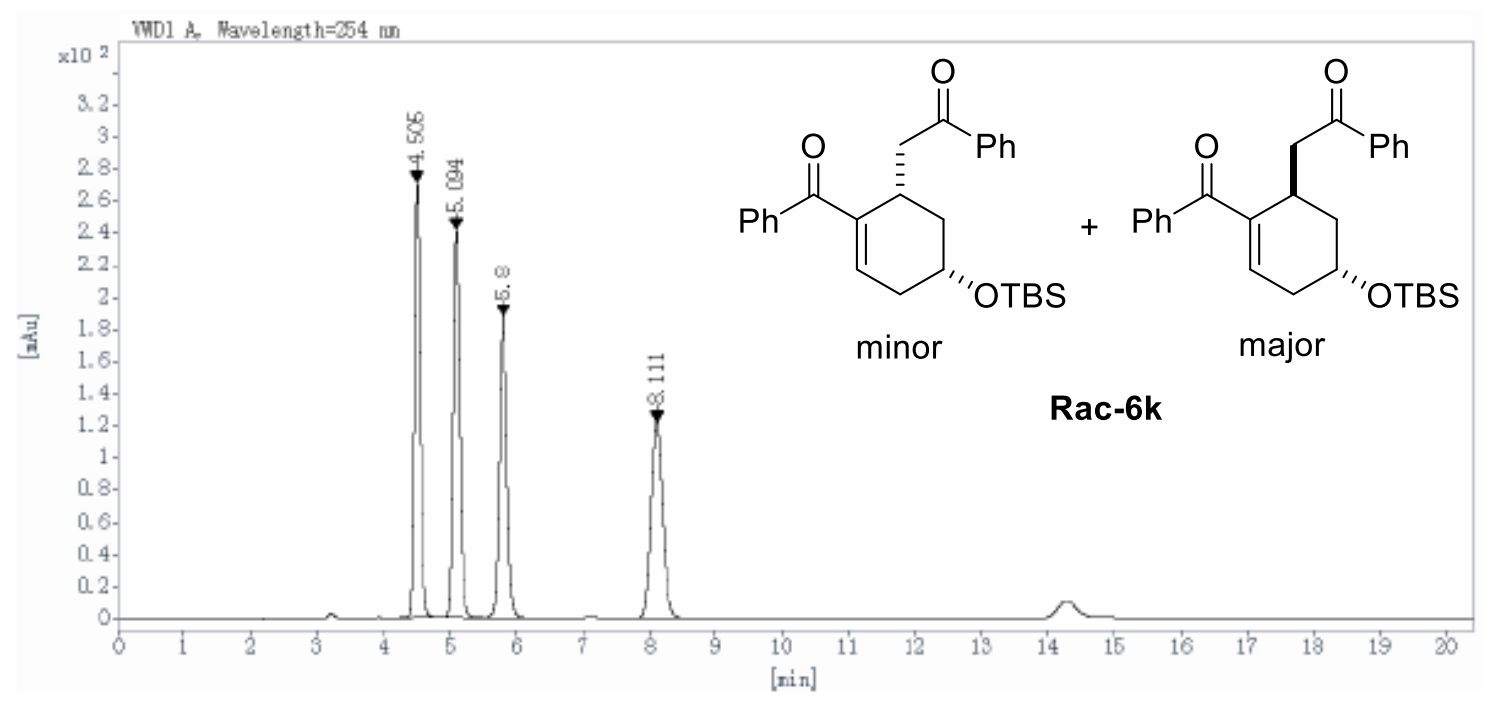

\begin{tabular}{cccccc}
$\begin{array}{c}\text { Ret Time } \\
\text { [min] }\end{array}$ & $\begin{array}{c}\text { Peak } \\
\text { Type }\end{array}$ & $\begin{array}{c}\text { Width } \\
{[\mathrm{min}]}\end{array}$ & $\begin{array}{c}\text { Height } \\
{[\mathrm{mAU}]}\end{array}$ & $\begin{array}{c}\text { Area } \\
{[\mathrm{mAU} * \text { s] }}\end{array}$ & $\begin{array}{c}\text { Area } \\
{[\%]}\end{array}$ \\
\hline 4.505 & VB R & 0.09 & 271.0721 & 1659.3181 & 26.2557 \\
5.094 & VV R & 0.11 & 241.3182 & 1664.2924 & 26.3344 \\
5.800 & BB & 0.12 & 187.5638 & 1511.5376 & 23.9174 \\
8.111 & BB & 0.19 & 122.4220 & 1484.6818 & 23.4924 \\
& & & Totals: & 6319.8298 & 100.0000
\end{tabular}

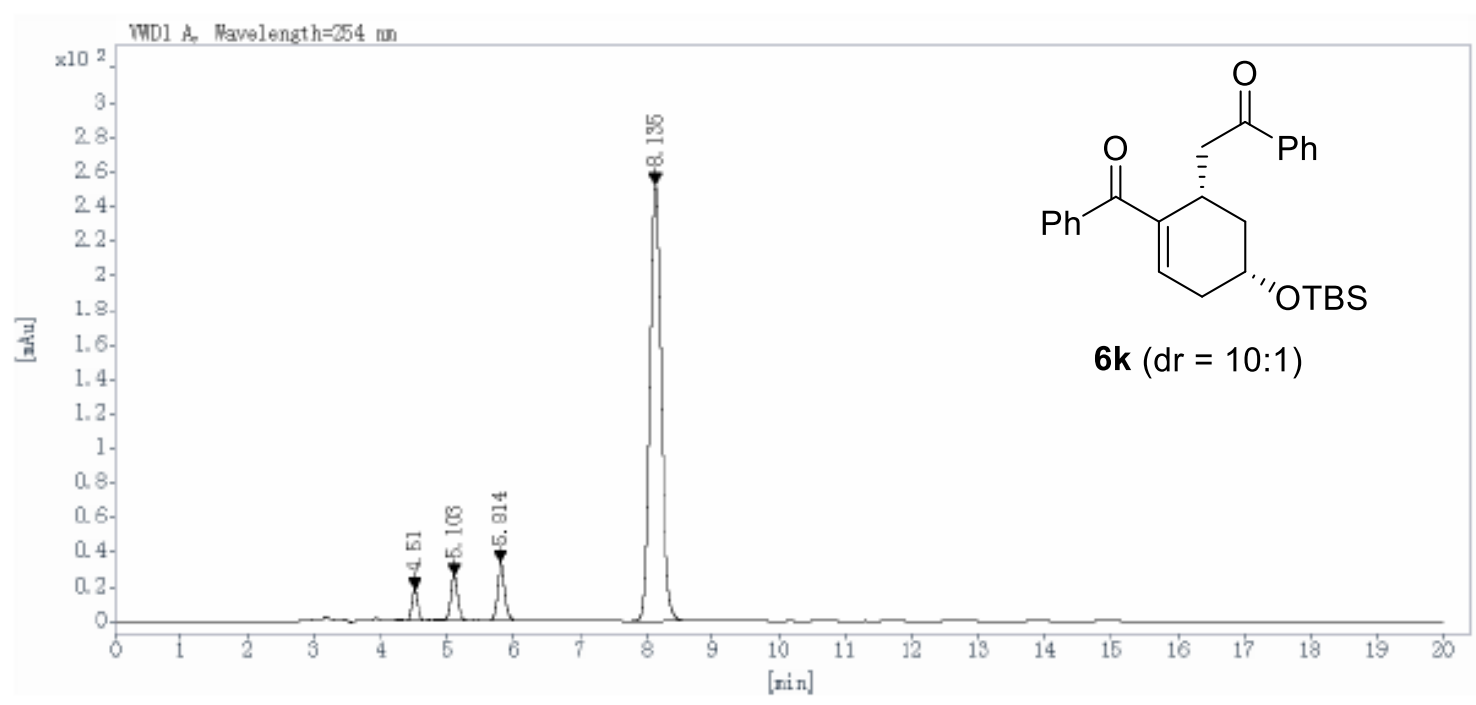

\begin{tabular}{cccccc}
$\begin{array}{c}\text { Ret Time } \\
{[\mathrm{min}]}\end{array}$ & $\begin{array}{c}\text { Peak } \\
\text { Type }\end{array}$ & $\begin{array}{c}\text { Width } \\
{[\mathrm{min}]}\end{array}$ & $\begin{array}{c}\text { Height } \\
{[\mathrm{mAU}]}\end{array}$ & $\begin{array}{c}\text { Area } \\
{\left[\mathrm{mAU}^{*} \text { s] }\right.}\end{array}$ & $\begin{array}{c}\text { Area } \\
{[\%]}\end{array}$ \\
\hline 4.510 & VB R & 0.10 & 17.5995 & 111.3081 & 3.1239 \\
5.103 & VB R & 0.11 & 25.9792 & 188.9893 & 5.3040 \\
5.814 & BB & 0.12 & 33.8162 & 273.0497 & 7.6631 \\
8.135 & BB & 0.18 & 252.5123 & 2989.8132 & 83.9090 \\
& & & Totals: & 3563.1604 & 100.0000
\end{tabular}


<smiles>CC(C)C[C@@H]1CC=C(C(=O)c2ccccc2)[C@H](CC(=O)c2ccccc2)C1</smiles>

6I

${ }^{1} \mathrm{H}$ NMR $\left(400 \mathrm{MHz}, \mathrm{CDCl}_{3}\right)$

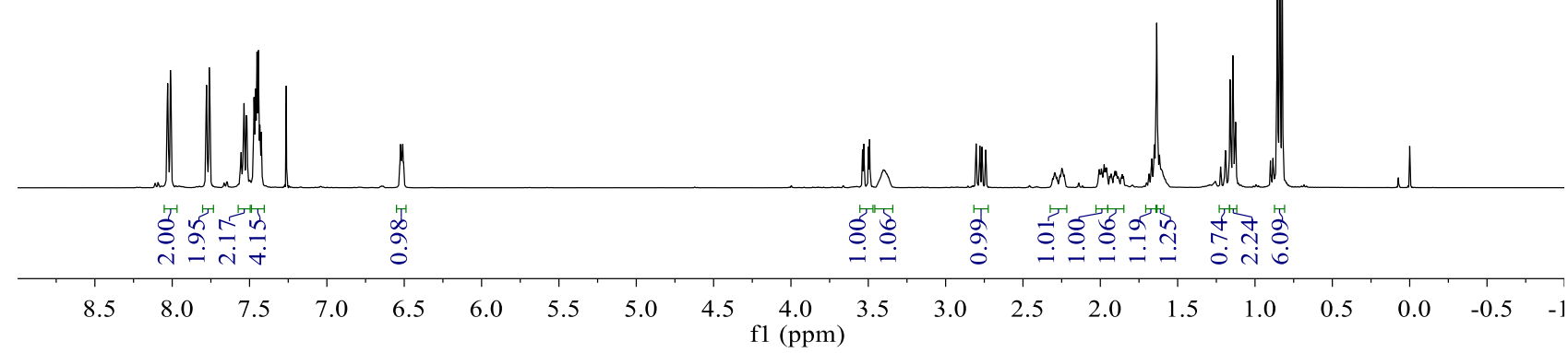

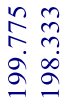

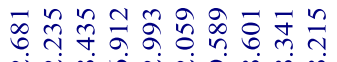

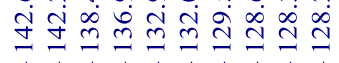

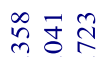

$\therefore$

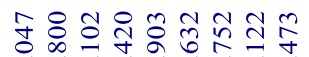

में<smiles>CC(C)C[C@@H]1CC=C(C(=O)c2ccccc2)[C@H](CC(=O)c2ccccc2)C1</smiles>

6I

${ }^{13} \mathrm{C} \mathrm{NMR}\left(100 \mathrm{MHz}, \mathrm{CDCl}_{3}\right)$

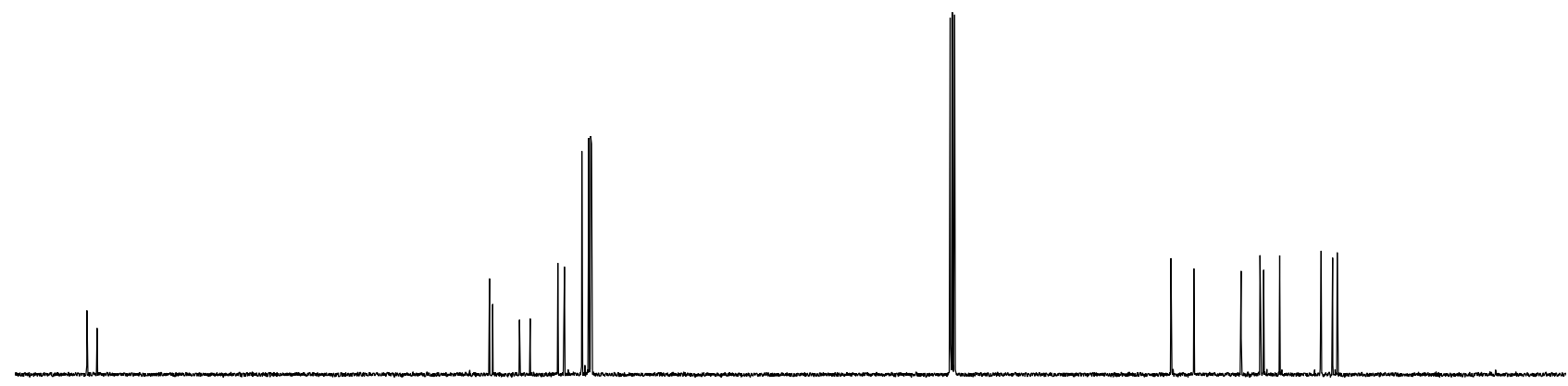

$\begin{array}{lllllllllllllllllllllll}10 & 200 & 190 & 180 & 170 & 160 & 150 & 140 & 130 & 120 & 110 & \begin{array}{c}100 \\ \mathrm{f} 1(\mathrm{ppm})\end{array} & 90 & 80 & 70 & 60 & 50 & 40 & 30 & 20 & 10 & 0 & -\end{array}$ 


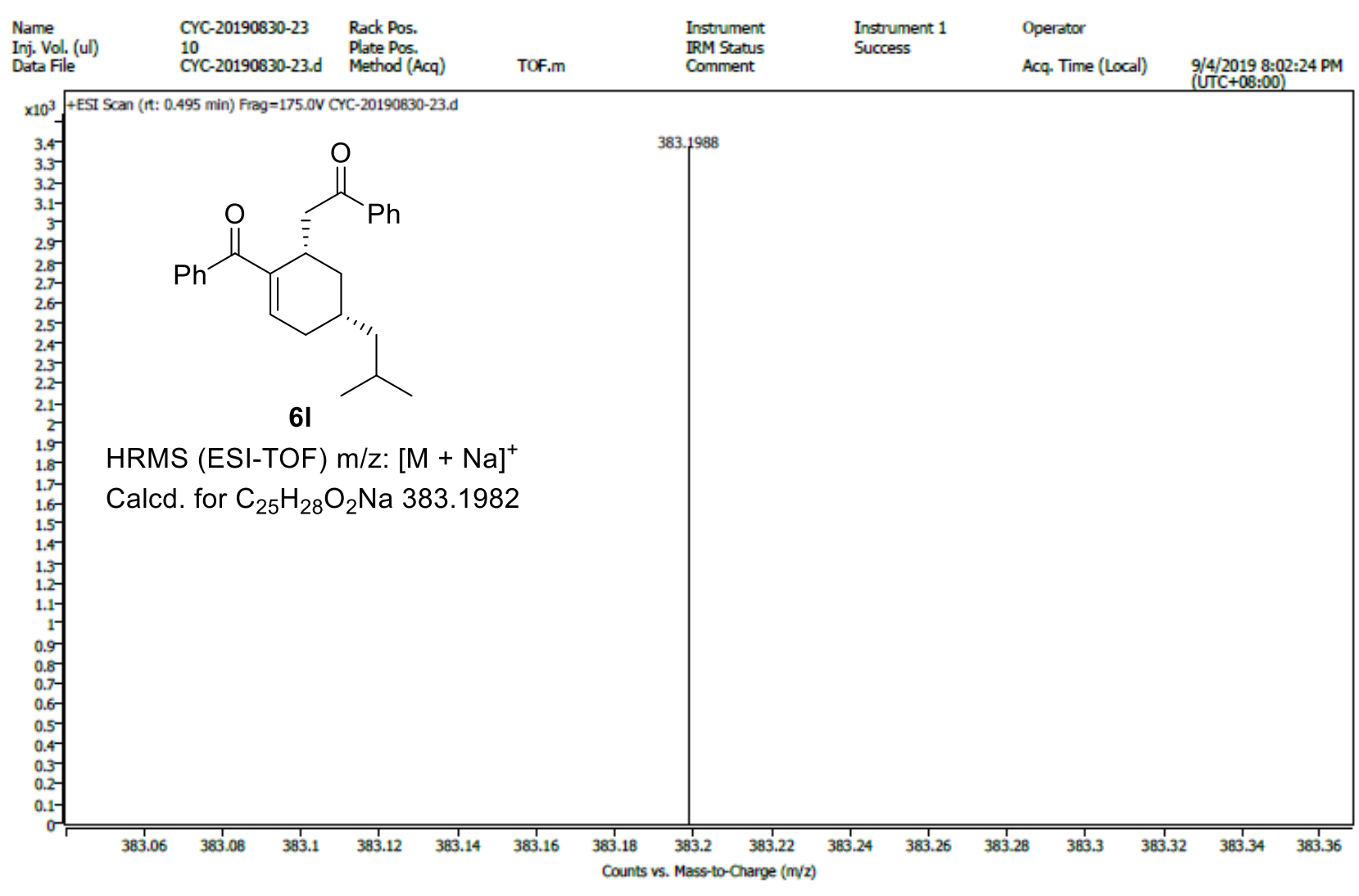




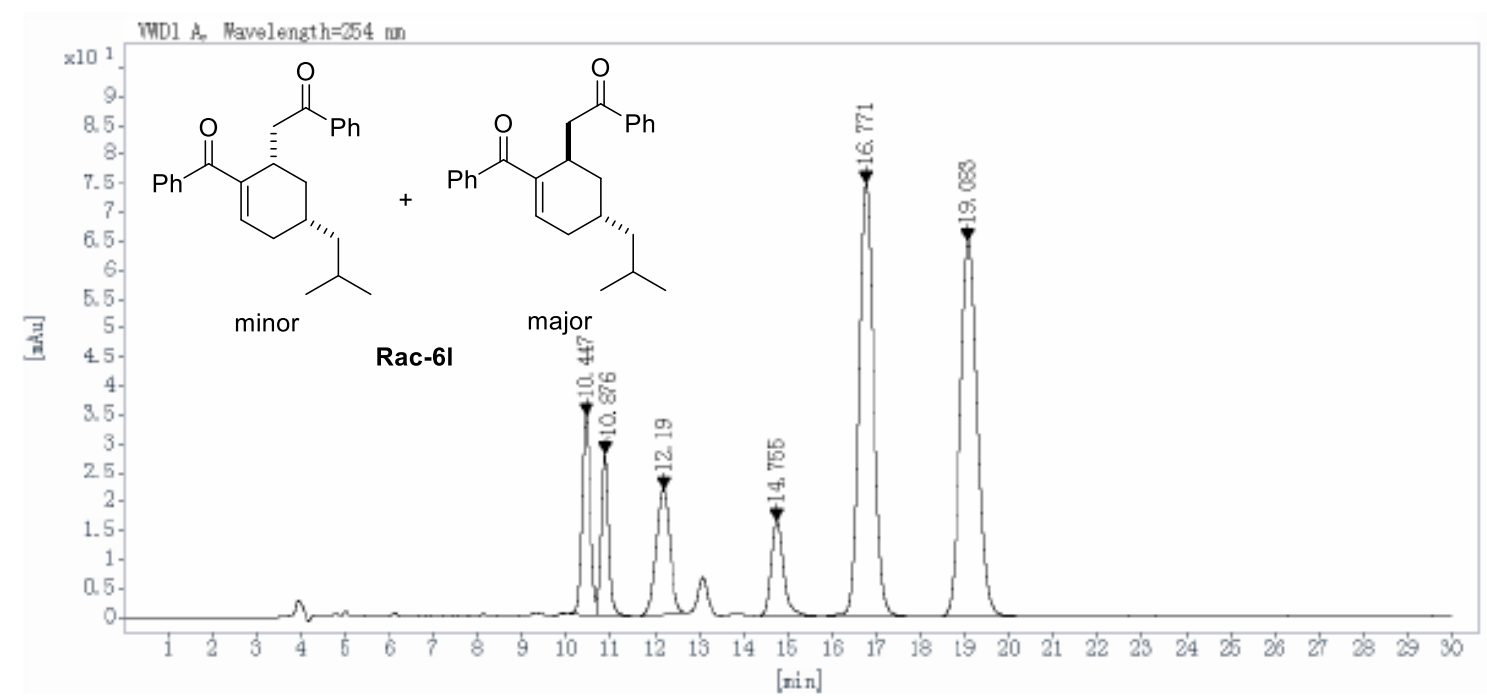

\begin{tabular}{cccccc}
$\begin{array}{c}\text { Ret Time } \\
\text { [min] }\end{array}$ & $\begin{array}{c}\text { Peak } \\
\text { Type }\end{array}$ & $\begin{array}{c}\text { Width } \\
{[\mathrm{min}]}\end{array}$ & $\begin{array}{c}\text { Height } \\
{[\mathrm{mAU}]}\end{array}$ & $\begin{array}{c}\text { Area } \\
{\left[\mathrm{mAU} \dot{*}^{\text {s] }}\right]}\end{array}$ & $\begin{array}{c}\text { Area } \\
{[\%]}\end{array}$ \\
\hline 10.447 & VV R & 0.21 & 34.5315 & 461.5835 & 9.2381 \\
10.876 & VB & 0.18 & 27.8887 & 319.3142 & 6.3907 \\
12.190 & BB & 0.33 & 21.6324 & 465.8922 & 9.3243 \\
14.755 & BB & 0.29 & 16.5441 & 314.7847 & 6.3001 \\
16.771 & BB & 0.36 & 74.8658 & 1722.6799 & 34.4775 \\
19.083 & BB & 0.41 & 64.8297 & 1712.2845 & 34.2694 \\
& & & Totals: & 4996.5390 & 100.0000
\end{tabular}

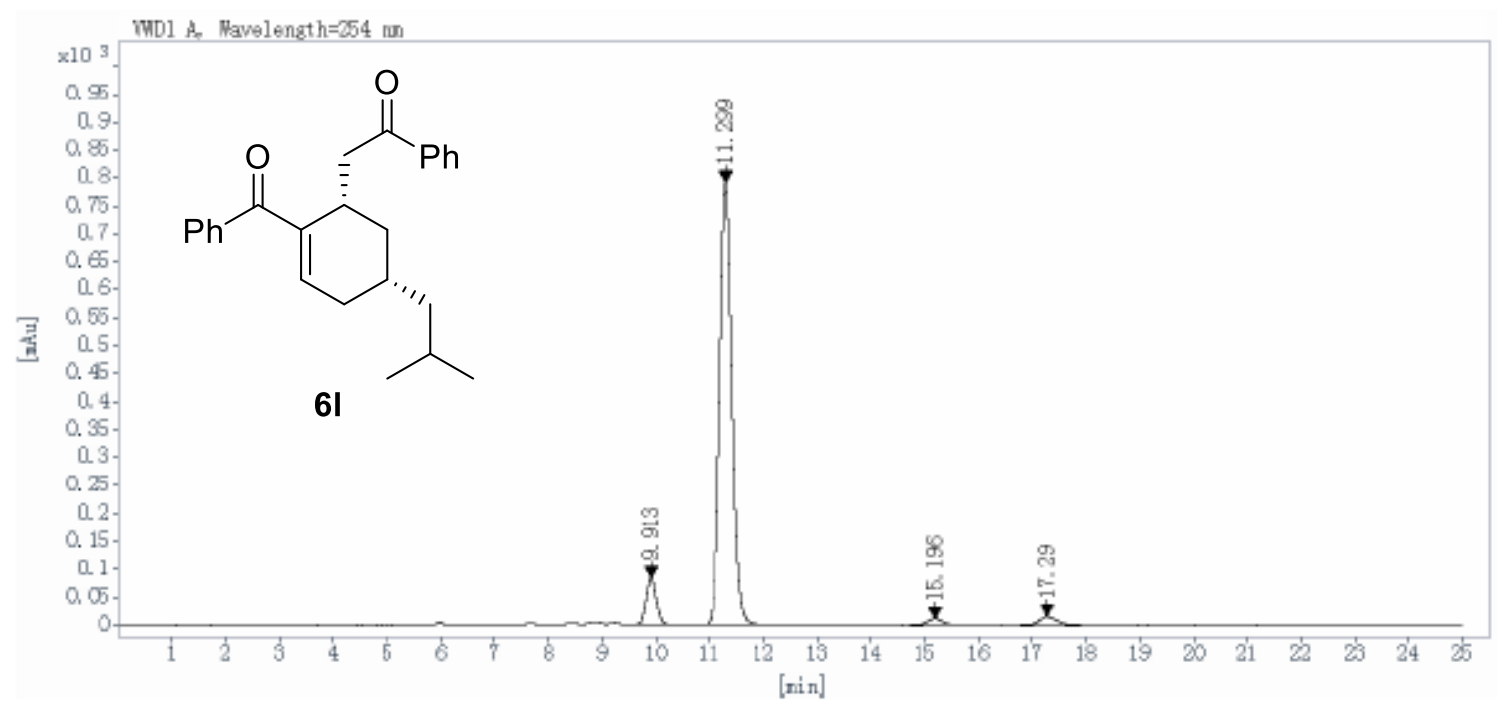

$\begin{array}{cccccc}\begin{array}{c}\text { Ret Time } \\ \text { [min] }\end{array} & \begin{array}{c}\text { Peak } \\ \text { Type }\end{array} & \begin{array}{c}\text { Width } \\ {[\mathbf{m i n}]}\end{array} & \begin{array}{c}\text { Height } \\ {[\mathrm{mAU}]}\end{array} & \begin{array}{c}\text { Area } \\ {[\mathbf{m A U} * \text { s] }}\end{array} & \begin{array}{c}\text { Area } \\ {[\%]}\end{array} \\ 9.913 & \text { BB } & 0.20 & 84.3493 & 1091.5378 & 7.7810 \\ 11.299 & \text { VB } & 0.24 & 790.8223 & 12355.9473 & 88.0794 \\ 15.196 & \text { BB } & 0.31 & 11.2611 & 228.0092 & 1.6254 \\ 17.290 & \text { BB } & 0.36 & 15.2646 & 352.6949 & 2.5142 \\ & & & \text { Totals: } & 14028.1892 & 100.0000\end{array}$


<smiles>O=C(C[C@@H]1CC=C([C@H]2CC=CCC2)[C@H](c2ccc(Cl)cc2)C1)c1ccc2ccccc2c1</smiles>

${ }^{1} \mathrm{H} \mathrm{NMR}\left(400 \mathrm{MHz}, \mathrm{CDCl}_{3}\right)$

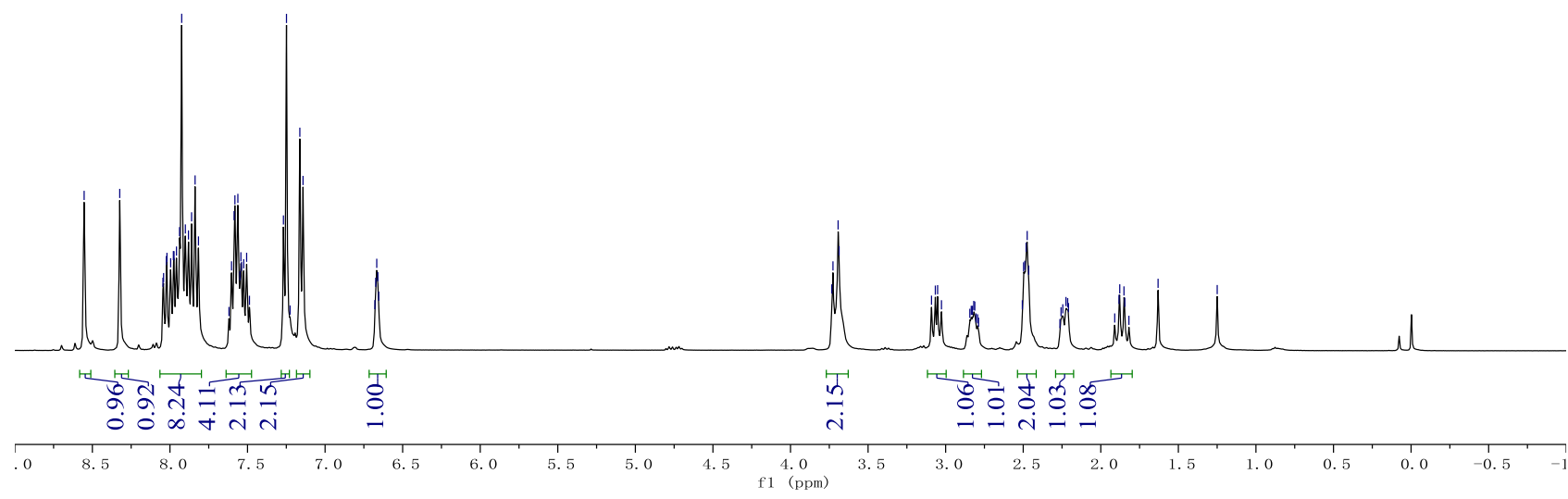

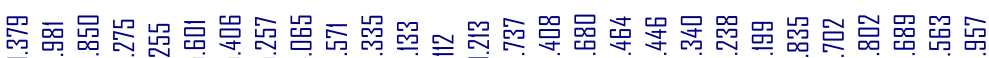

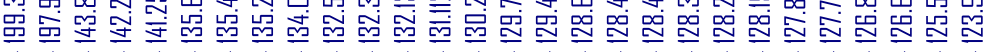

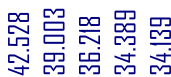<smiles>O=C(C[C@@H]1CC=C(C(=O)c2ccc3ccccc3c2)[C@H](c2ccc(Cl)cc2)C1)c1ccc2ccccc2c1</smiles>

$6 m$

${ }^{13} \mathrm{C}$ NMR $\left(100 \mathrm{MHz}, \mathrm{CDCl}_{3}\right)$

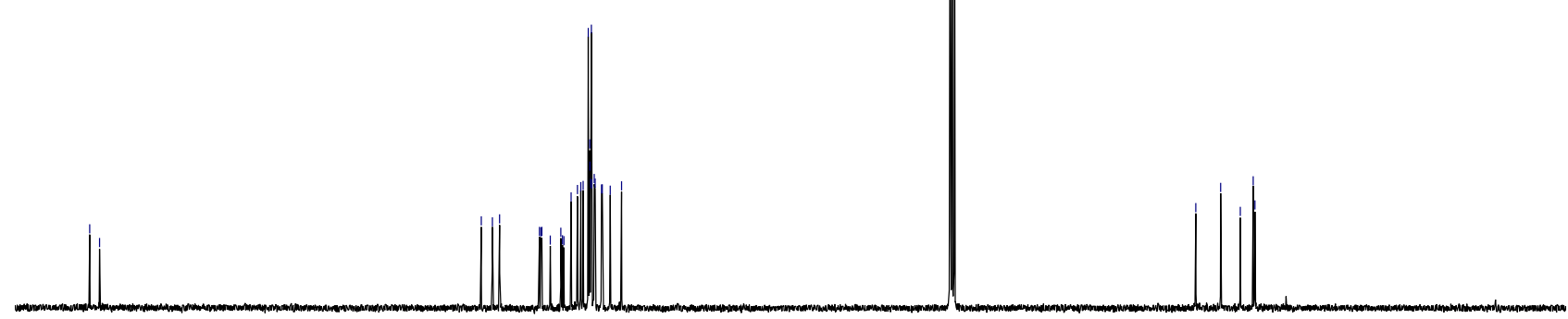



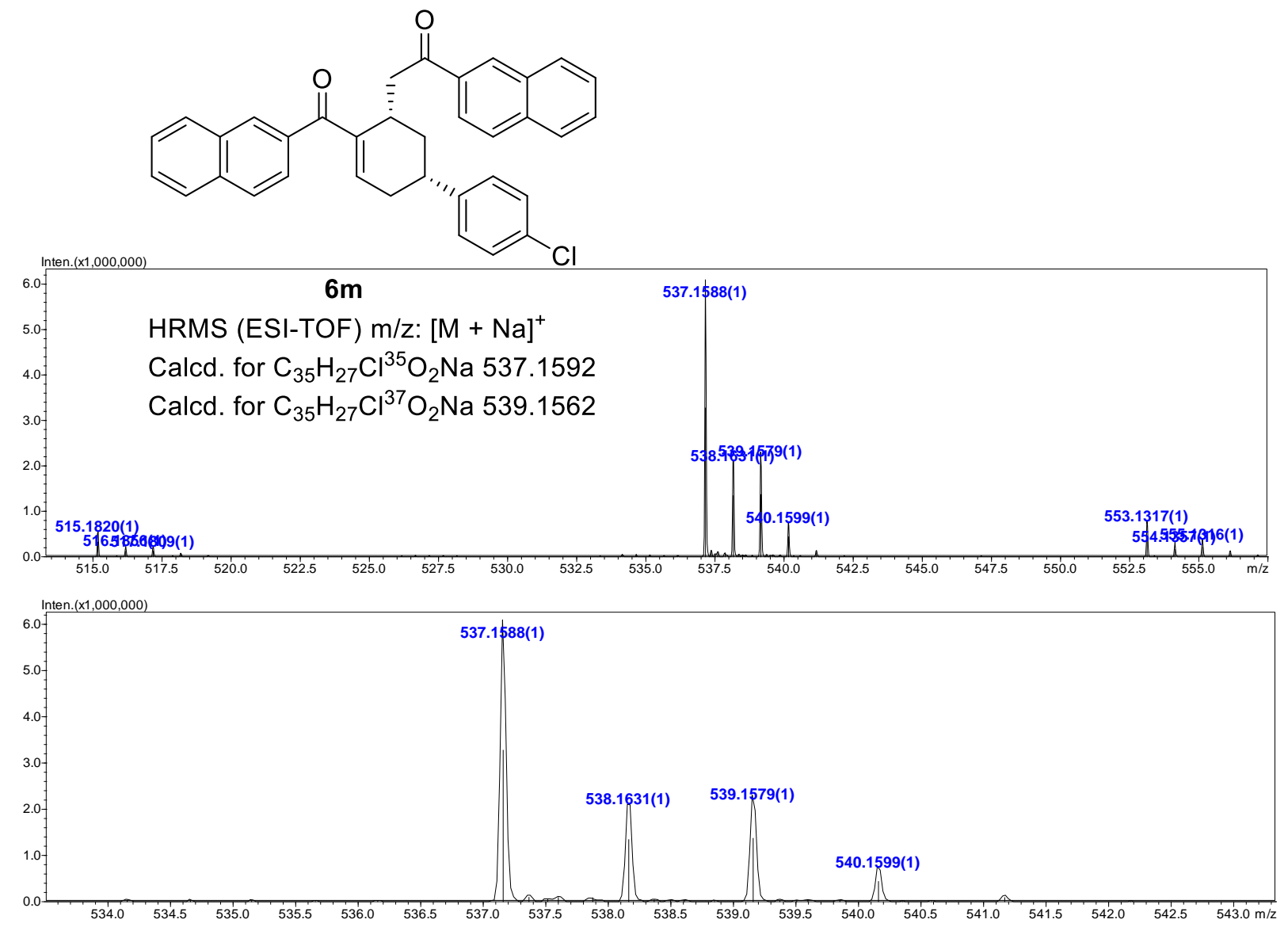


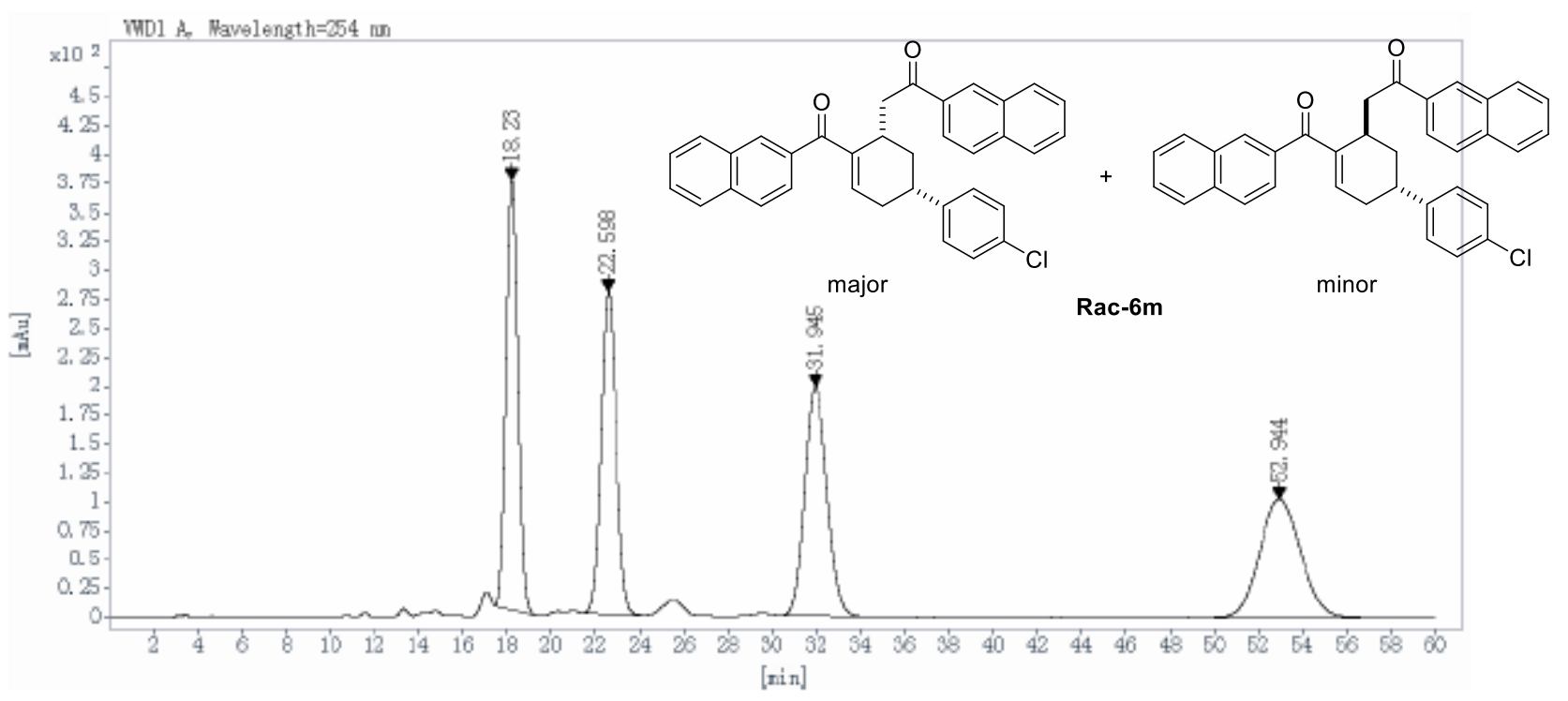

$\begin{array}{cccccc}\begin{array}{c}\text { Ret Time } \\ \text { [min] }\end{array} & \begin{array}{c}\text { Peak } \\ \text { Type }\end{array} & \begin{array}{c}\text { Width } \\ {[\mathrm{min}]}\end{array} & \begin{array}{c}\text { Height } \\ {[\mathrm{mAU}]}\end{array} & \begin{array}{c}\text { Area } \\ {[\mathrm{mAU} * \mathrm{~s}]}\end{array} & \begin{array}{c}\text { Area } \\ {[\%]}\end{array} \\ 18.230 & \text { BB } & 0.58 & 371.7292 & 13644.1963 & 25.3179 \\ 22.598 & \text { BB } & 0.73 & 279.2758 & 13143.5674 & 24.3890 \\ 31.945 & \text { BB } & 1.10 & 198.3383 & 13953.7695 & 25.8924 \\ 52.944 & \text { BB } & 1.98 & 102.6664 & 13149.8750 & 24.4007 \\ & & & \text { Totals: } & 53891.4082 & 100.0000\end{array}$

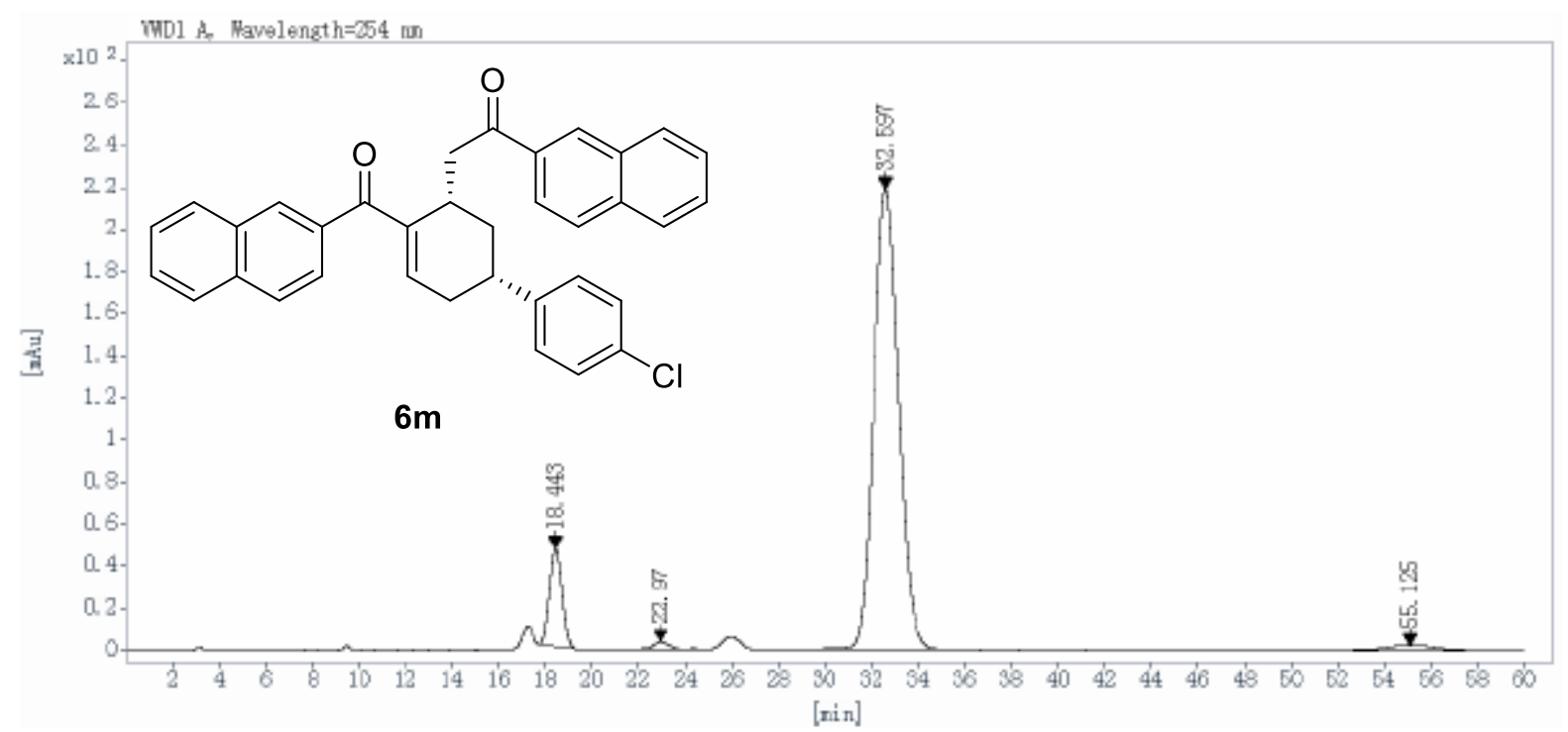

$\begin{array}{cccccc}\begin{array}{c}\text { Ret Time } \\ \text { [min] }\end{array} & \begin{array}{c}\text { Peak } \\ \text { Type }\end{array} & \begin{array}{c}\text { Width } \\ {[\mathrm{min}]}\end{array} & \begin{array}{c}\text { Height } \\ {[\mathrm{mAU}]}\end{array} & \begin{array}{c}\text { Area } \\ {[\mathrm{mAU} \text { *s] }}\end{array} & \begin{array}{c}\text { Area } \\ {[\%]}\end{array} \\ 18.443 & \text { BB } & 0.58 & 46.7062 & 1716.3251 & 9.2832 \\ 22.970 & \text { BV R } & 0.82 & 3.8235 & 213.9149 & 1.1570 \\ 32.597 & \text { VB R } & 1.16 & 218.6123 & 16239.7969 & 87.8376 \\ 55.125 & \text { BB } & 1.56 & 2.4144 & 318.4016 & 1.7222 \\ & & & \text { Totals: } & 18488.4385 & 100.0000\end{array}$


<smiles>CCOC(=O)C1(C#N)CC=C(C(=O)c2ccccc2)C(CC(=O)c2ccccc2)C1</smiles>

8

${ }^{1} \mathrm{H} \mathrm{NMR}\left(400 \mathrm{MHz}, \mathrm{CDCl}_{3}\right)$

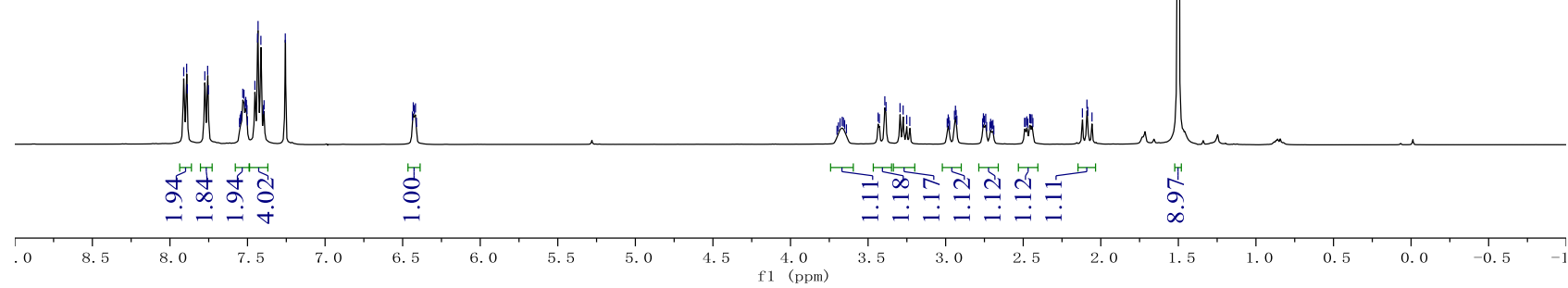

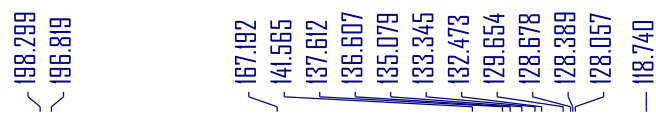

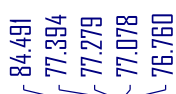

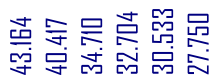<smiles>CCOC(=O)[C@]1(C#N)CC=C(C(=O)c2ccccc2)[C@H](CC(=O)c2ccccc2)C1</smiles>

8

${ }^{13} \mathrm{C}$ NMR $\left(400 \mathrm{MHz}, \mathrm{CDCl}_{3}\right)$
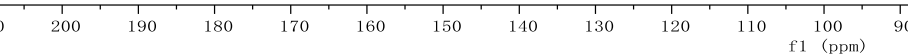


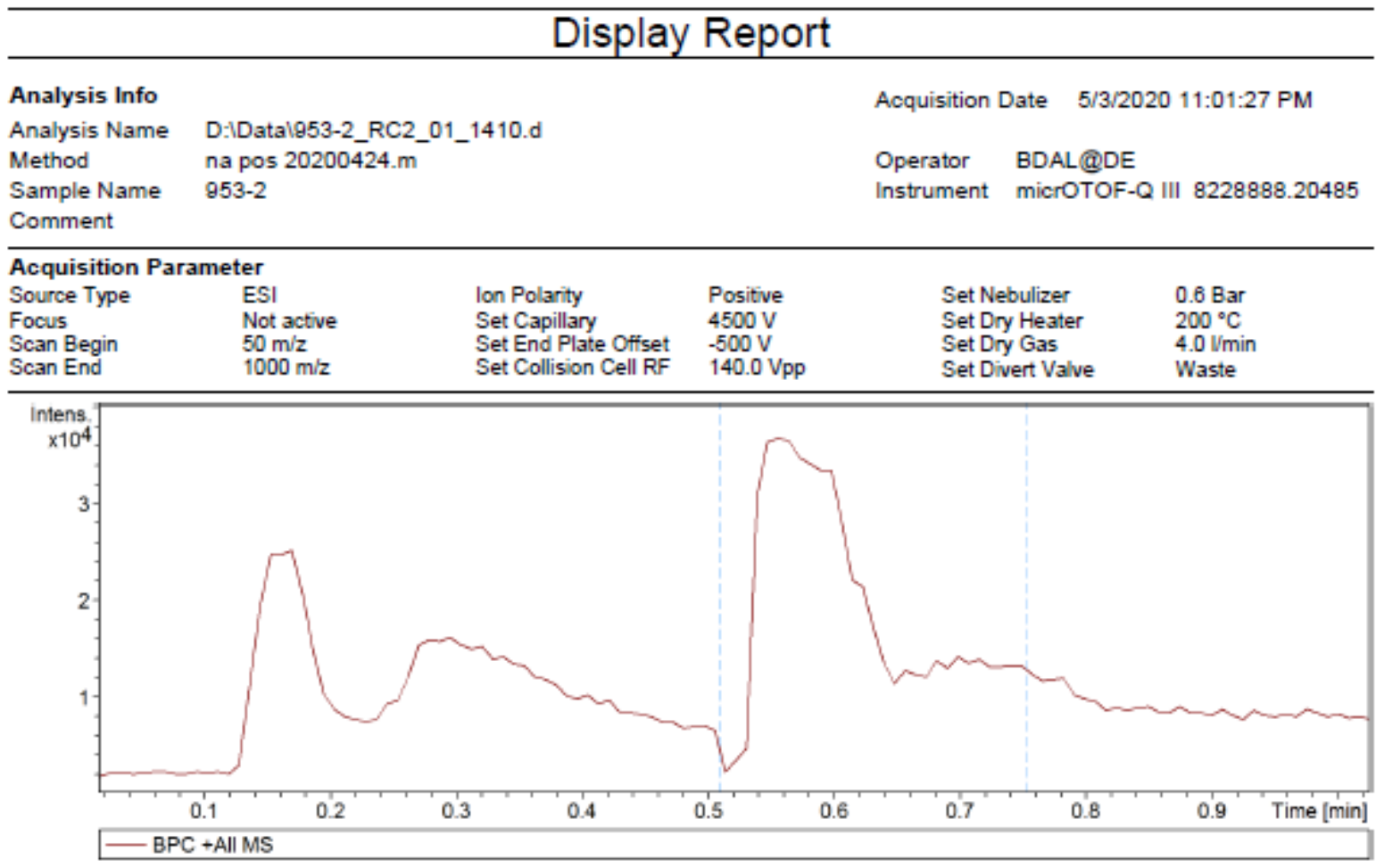
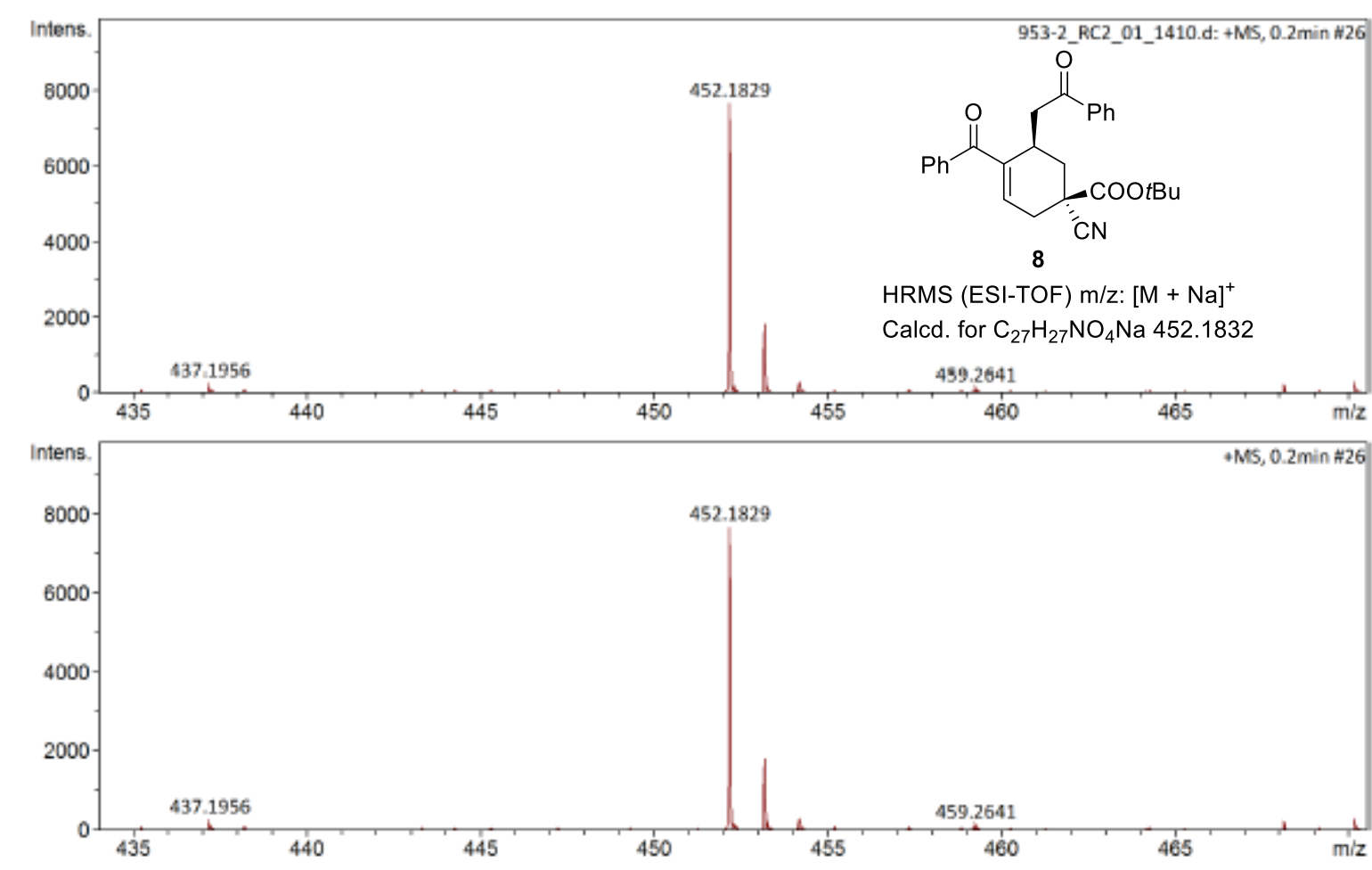


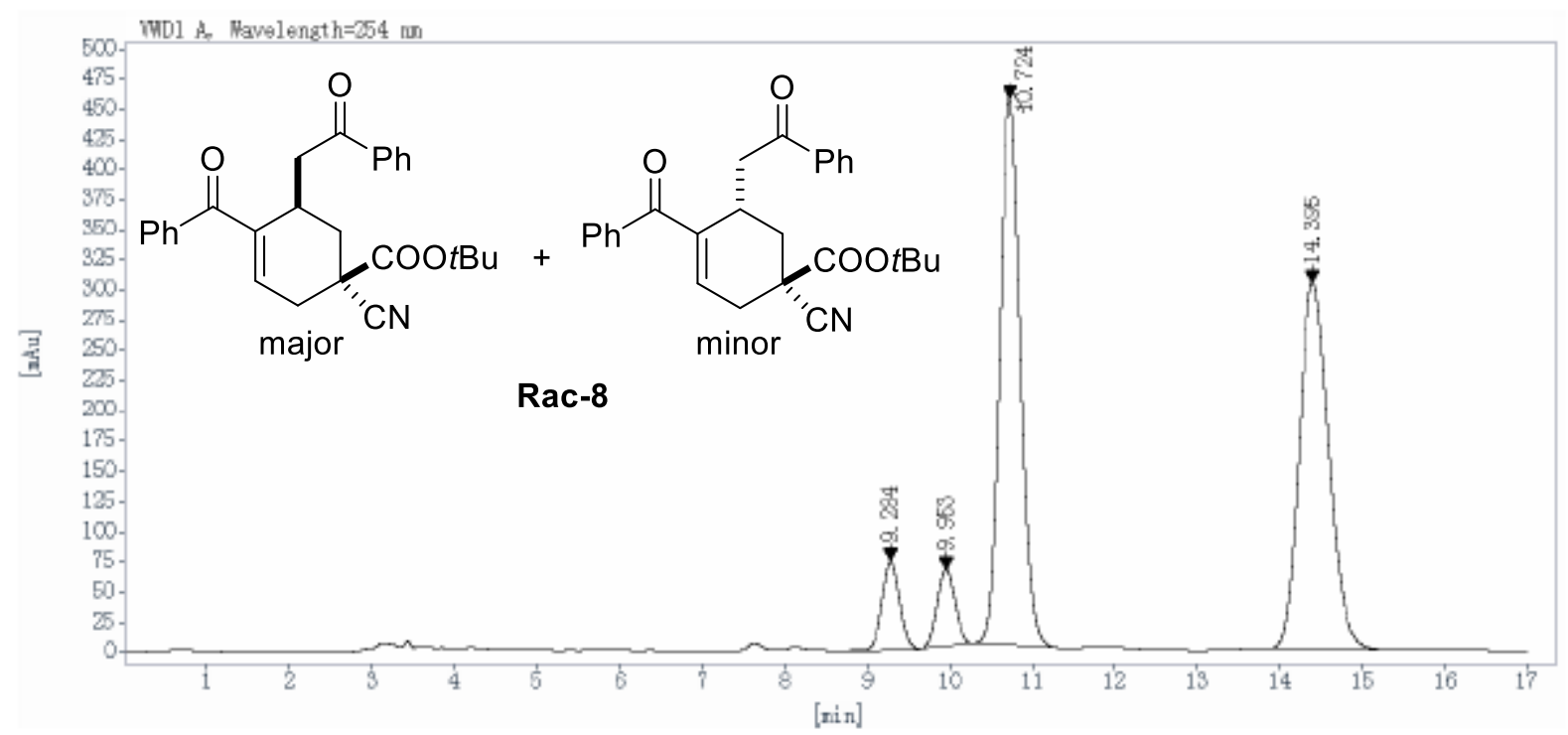

$\begin{array}{cccccc}\begin{array}{c}\text { Ret Time } \\ \text { [min] }\end{array} & \begin{array}{c}\text { Peak } \\ \text { Type }\end{array} & \begin{array}{c}\text { Width } \\ \text { [min] }\end{array} & \begin{array}{c}\text { Height } \\ {[\mathrm{mAU}]}\end{array} & \begin{array}{c}\text { Area } \\ {\left[\mathrm{mAU} \mathbf{U}^{*} \text { s] }\right.}\end{array} & \begin{array}{c}\text { Area } \\ {[\%]}\end{array} \\ 9.284 & \text { BB } & 0.23 & 73.5074 & 1071.5033 & 6.1247 \\ 9.953 & \text { BBA } & 0.23 & 64.1928 & 955.6726 & 5.4626 \\ 10.724 & \text { BBA } & 0.27 & 454.2599 & 7866.8188 & 44.9665 \\ 14.395 & \text { BB } & 0.39 & 304.8673 & 7600.8564 & 43.4462 \\ & & & \text { Totals: } & 17494.8512 & 100.0000\end{array}$

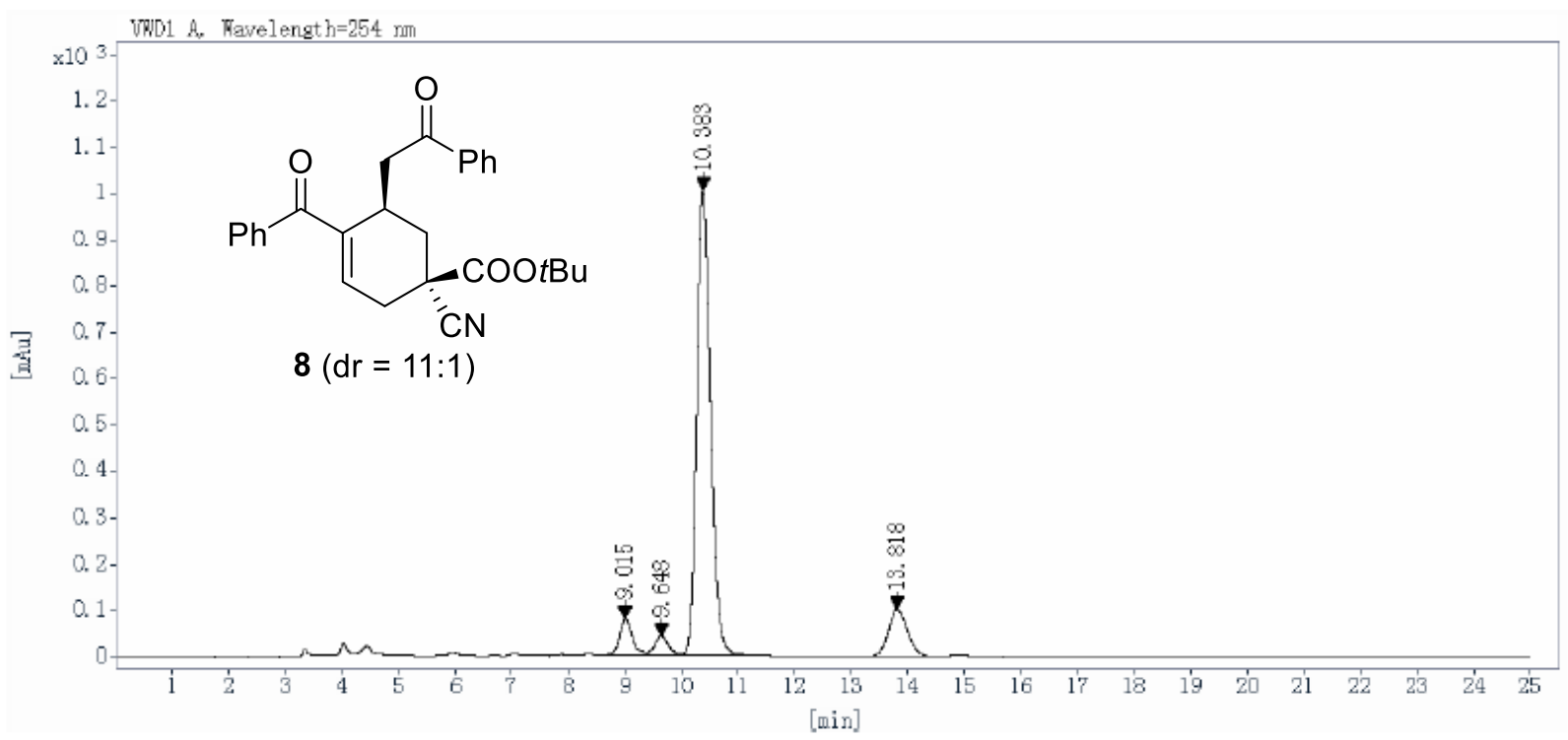

$\begin{array}{cccccc}\begin{array}{c}\text { Ret Time } \\ \text { [min] }\end{array} & \begin{array}{c}\text { Peak } \\ \text { Type }\end{array} & \begin{array}{c}\text { Width } \\ \text { [min] }\end{array} & \begin{array}{c}\text { Height } \\ {[\mathrm{mAU}]}\end{array} & \begin{array}{c}\text { Area } \\ {\left[\mathrm{mAU} \mathbf{U}^{*} \text { ] }\right]}\end{array} & \begin{array}{c}\text { Area } \\ {[\%]}\end{array} \\ 9.015 & \text { BV E } & 0.23 & 78.0662 & 1176.2234 & 5.5193 \\ 9.648 & \text { VV E } & 0.25 & 41.8186 & 691.4473 & 3.2445 \\ 10.383 & \text { VB R } & 0.26 & 1003.8346 & 17039.9883 & 79.9579 \\ 13.818 & \text { BB } & 0.36 & \begin{array}{c}102.0132 \\ \text { Totals: }\end{array} & 2403.5505 & 11.2783 \\ & & & & & \end{array}$


<smiles>O=C(C[C@H]1C[C@H]2CC[C@@H]1C=C2c1ccccc1)c1ccccc1</smiles>

10

${ }^{1} \mathrm{H}$ NMR $\left(400 \mathrm{MHz}, \mathrm{CDCl}_{3}\right)$

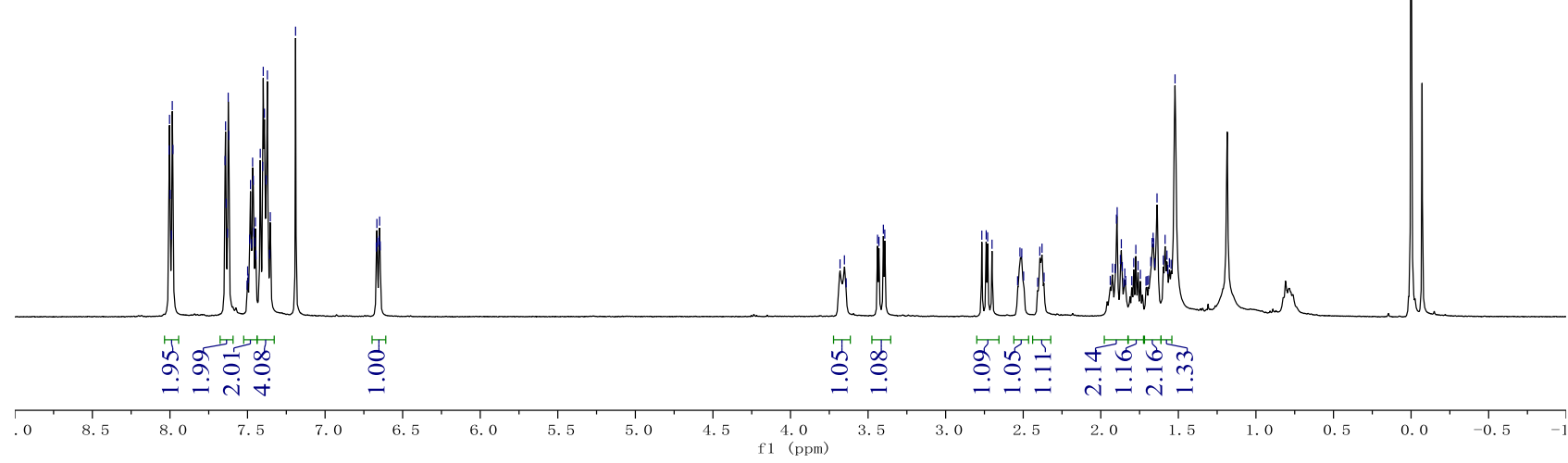

㗊萜

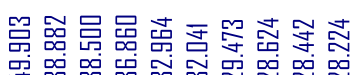

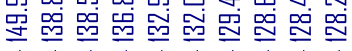

然

$1,1,-1$<smiles>O=C(C[C@H]1C[C@H]2CC[C@@H]1C=C2c1ccccc1)c1ccccc1</smiles>

10

${ }^{13} \mathrm{C}$ NMR $\left(100 \mathrm{MHz}, \mathrm{CDCl}_{3}\right)$

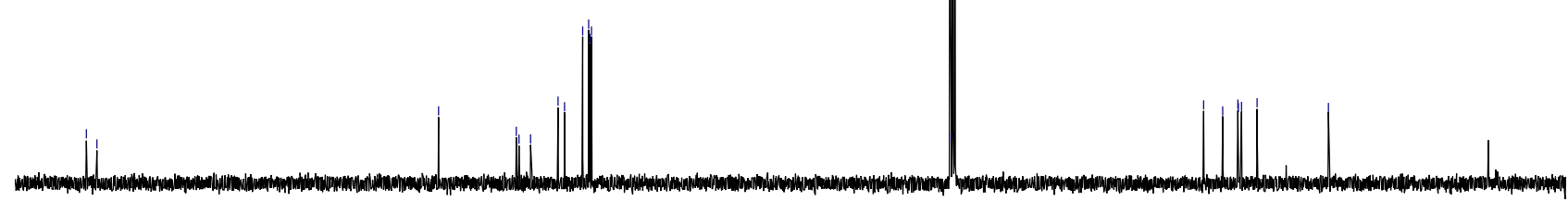

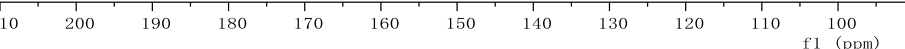




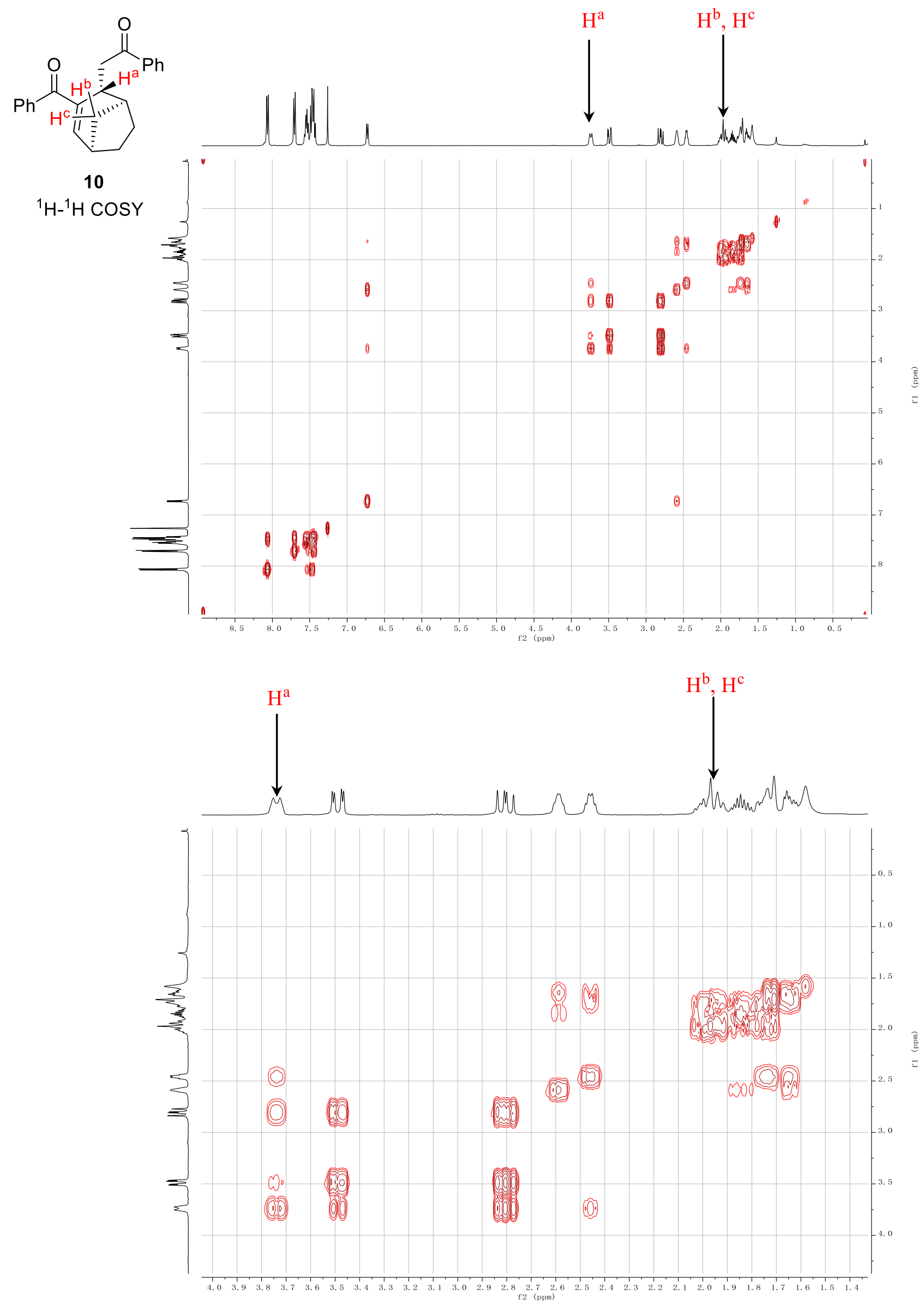


<smiles>O=C(C[C@@H]1C[C@@H]2CC[C@H]1C[C@@H]2C(=O)c1ccccc1)c1ccccc1</smiles>

10, NOEDS, $3.73 \mathrm{ppm}$ NOEDS NMR $\left(600 \mathrm{MHz}, \mathrm{CDCl}_{3}\right)$ irradiation $\mathrm{H}^{\mathrm{a}}$ at $3.73 \mathrm{ppm}$ no $\mathrm{H}^{\mathrm{b}}, \mathrm{H}^{\mathrm{c}}$ signal at $1.97 \mathrm{ppm}$

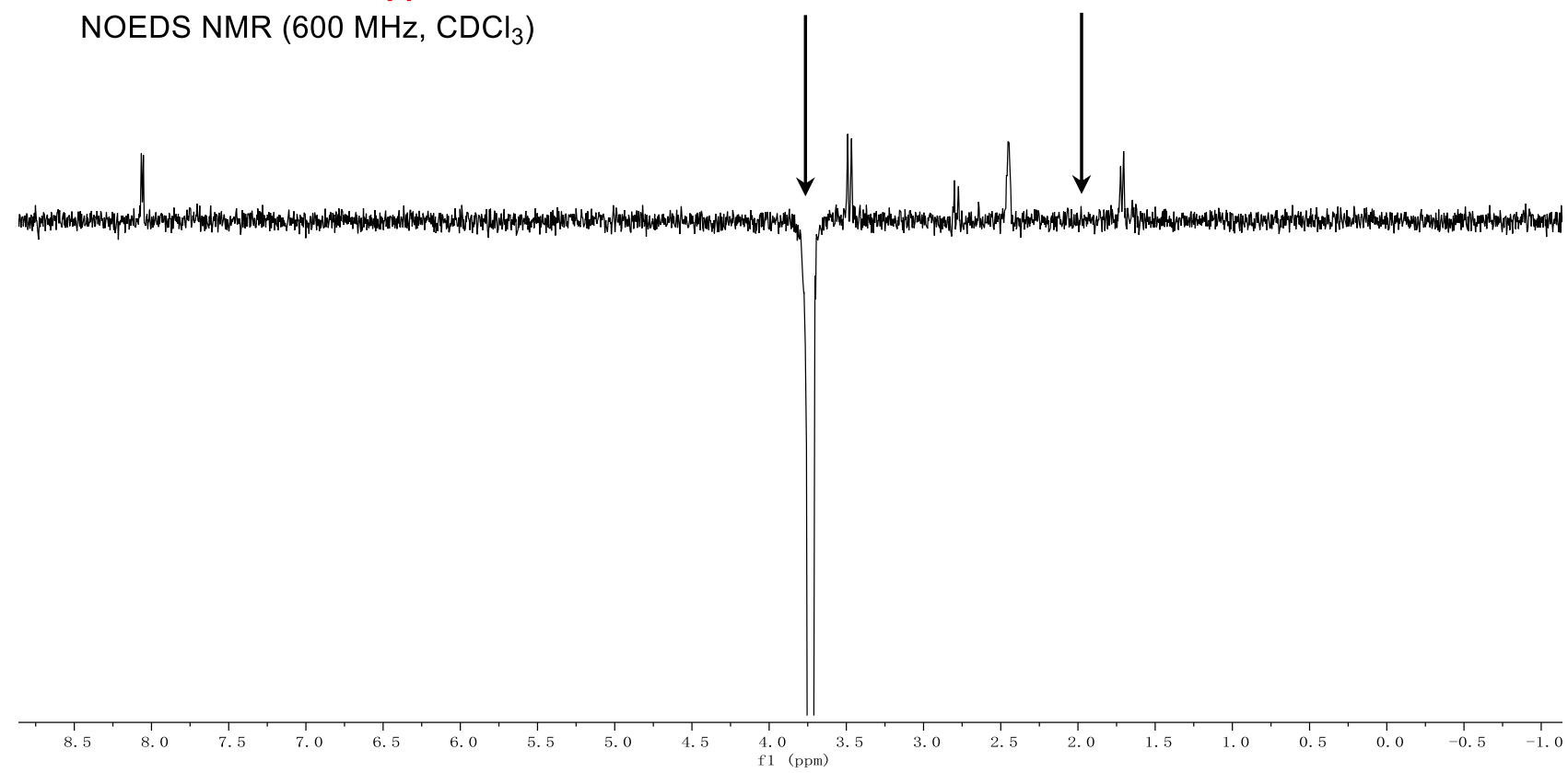

JY-359-a 14 (0.177)<smiles>O=C(CC1CC=CC1=C(C(=O)c1ccccc1)C1CC2CCC1CC2)c1ccccc1</smiles>

10

HRMS (ESI-TOF) m/z: [M + Na ${ }^{+}$

Calcd. for $\mathrm{C}_{23} \mathrm{H}_{22} \mathrm{O}_{2} \mathrm{Na} 353.1512$

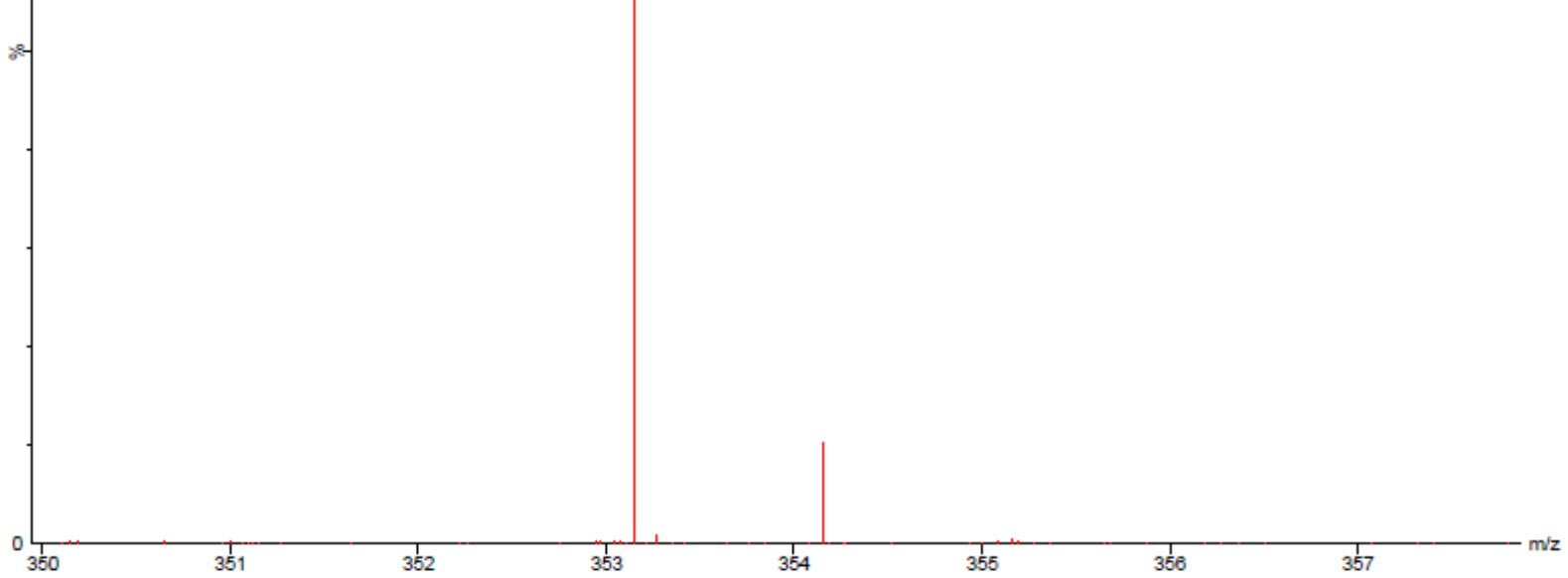




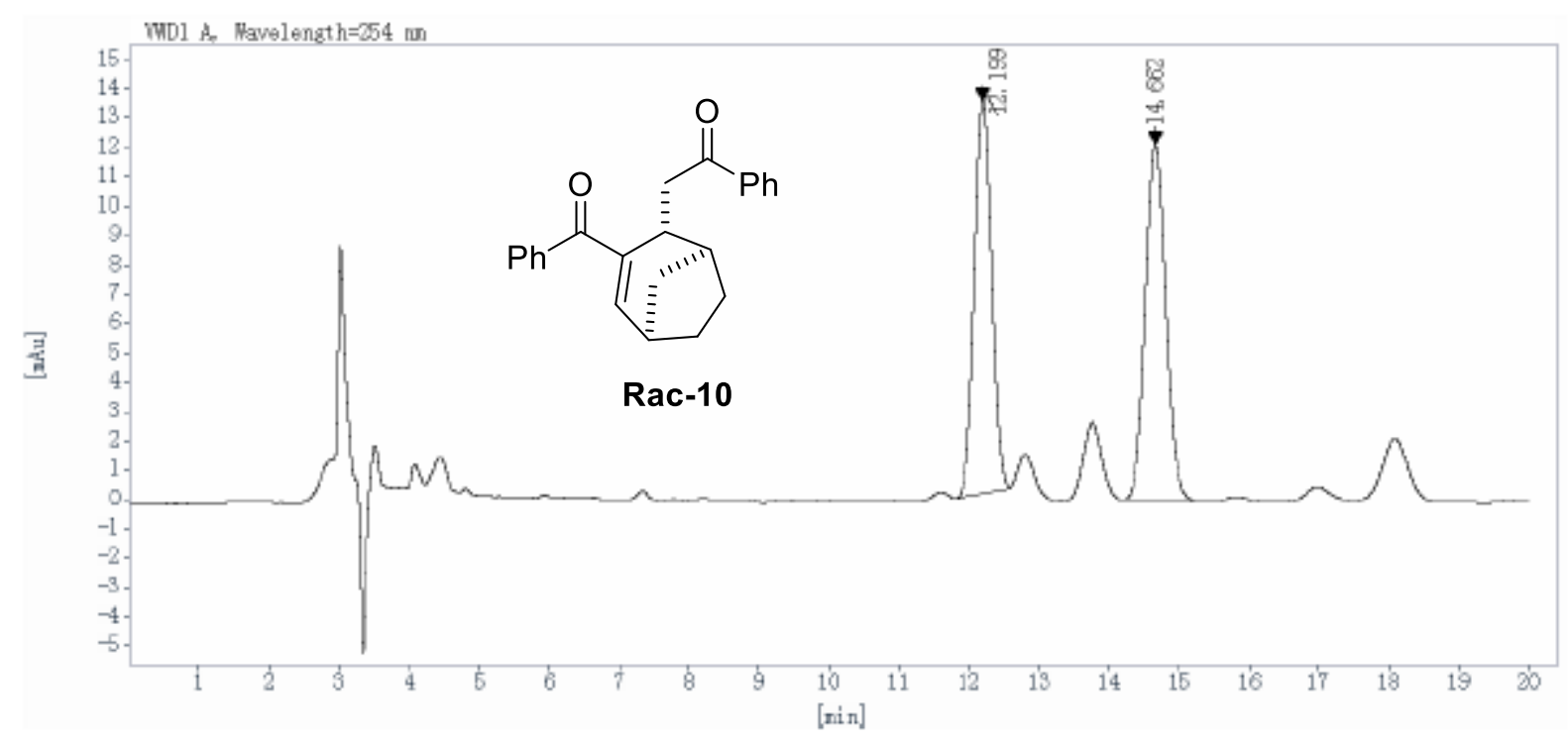

\begin{tabular}{|c|c|c|c|c|c|}
\hline $\begin{array}{l}\text { Ret Time } \\
\text { [min] }\end{array}$ & $\begin{array}{l}\text { Peak } \\
\text { Type }\end{array}$ & $\begin{array}{l}\text { Width } \\
\text { [min] }\end{array}$ & $\begin{array}{l}\text { Height } \\
{[\mathrm{mAU}]}\end{array}$ & $\begin{array}{c}\text { Area } \\
{\left[\mathrm{mAU}^{*} *_{s}\right]}\end{array}$ & $\begin{array}{l}\text { Area } \\
{[\%]}\end{array}$ \\
\hline 12.199 & BB & 0.26 & 13.4219 & 227.3423 & 48.1783 \\
\hline 14.662 & BB & 0.31 & 12.1400 & 244.5347 & 51.8217 \\
\hline & & & Totals: & 471.8770 & 100.0000 \\
\hline
\end{tabular}

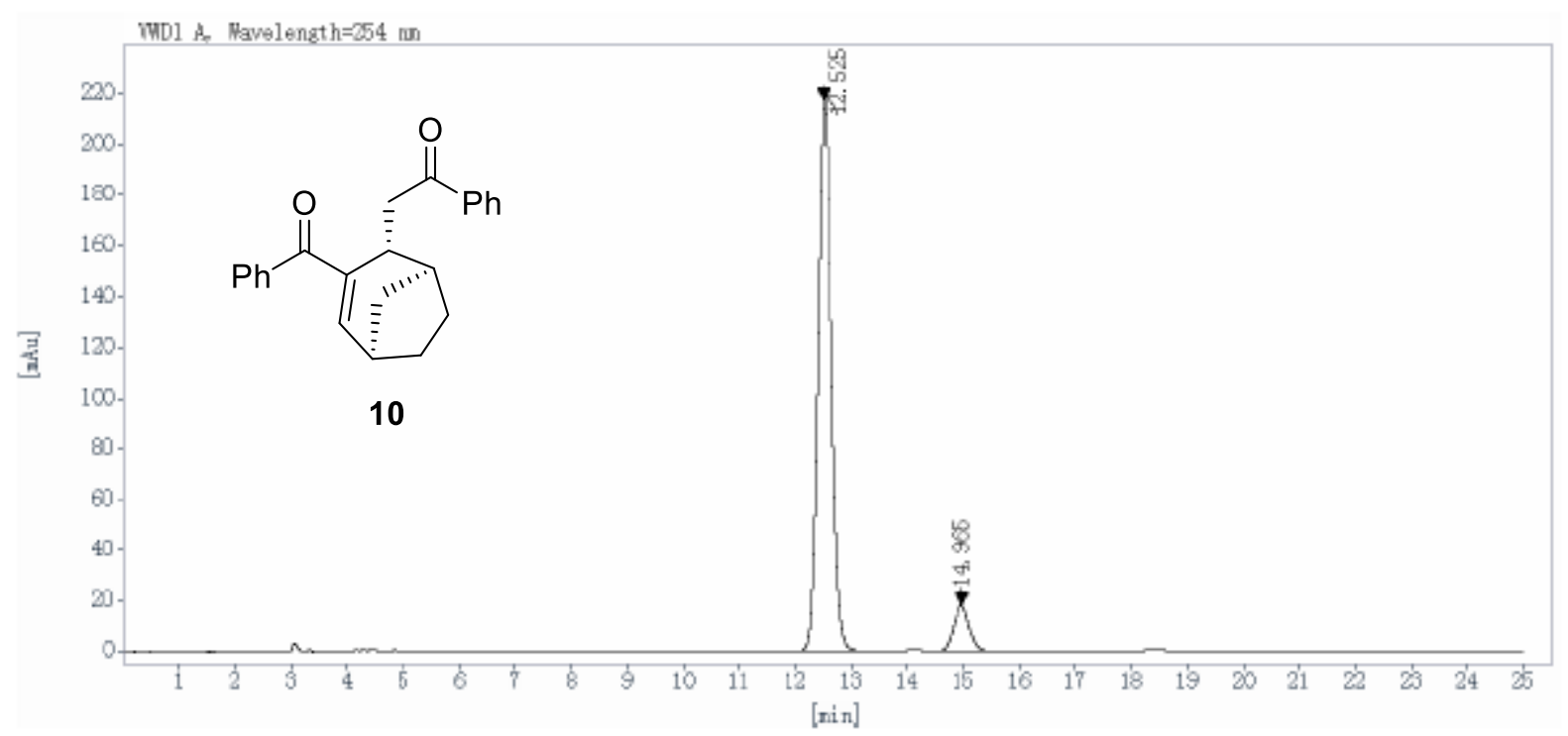

\begin{tabular}{cccccc}
$\begin{array}{c}\text { Ret Time } \\
\text { [min] }\end{array}$ & $\begin{array}{c}\text { Peak } \\
\text { Type }\end{array}$ & $\begin{array}{c}\text { Width } \\
{[\mathrm{min}]}\end{array}$ & $\begin{array}{c}\text { Height } \\
{[\mathrm{mAU}]}\end{array}$ & $\begin{array}{c}\text { Area } \\
{\left[\mathrm{mAU} \mathbf{*}^{*}\right]}\end{array}$ & $\begin{array}{c}\text { Area } \\
{[\%]}\end{array}$ \\
\hline 12.525 & BB & 0.26 & 217.7137 & 3639.0811 & 90.9940 \\
14.965 & BB & 0.30 & 18.3207 & 360.1709 & 9.0060 \\
& & & Totals: & 3999.2520 & 100.0000 \\
& & & & &
\end{tabular}




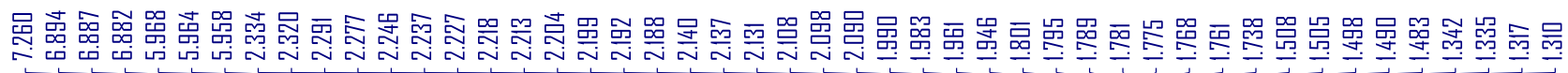<smiles>CC1=CC(=O)C2=CCCC[C@H]2C1</smiles>

11

${ }^{1} \mathrm{H}$ NMR $(400 \mathrm{MHz}, \mathrm{CDCl} 3)$

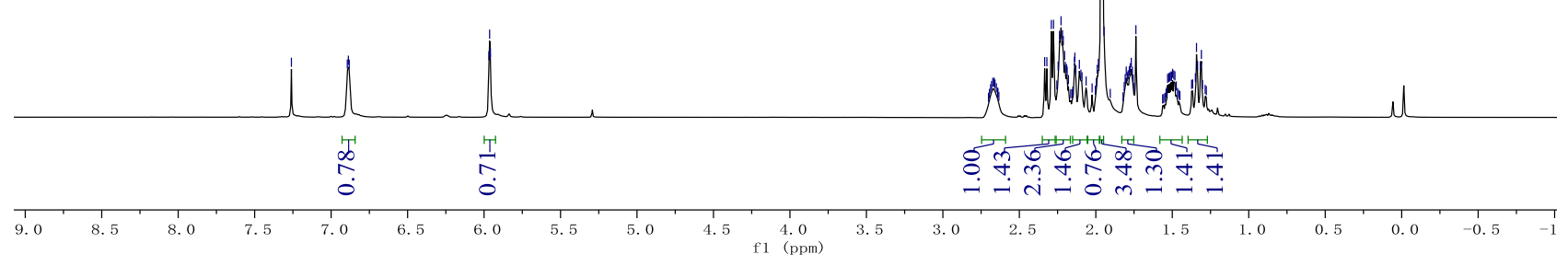

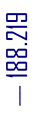

$\frac{\text { 罯 }}{\text { । }}$

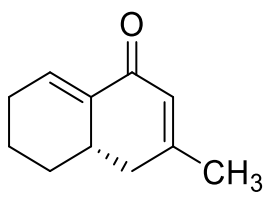

11

${ }^{13} \mathrm{C}$ NMR $(100 \mathrm{MHz}, \mathrm{CDCl} 3)$
量㽞哭

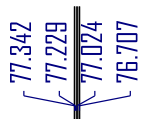

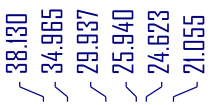
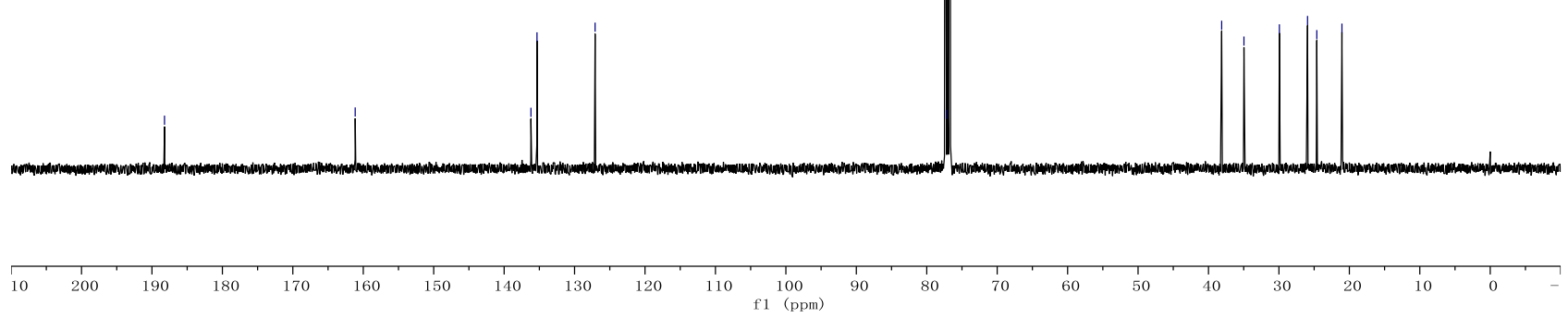

S146 


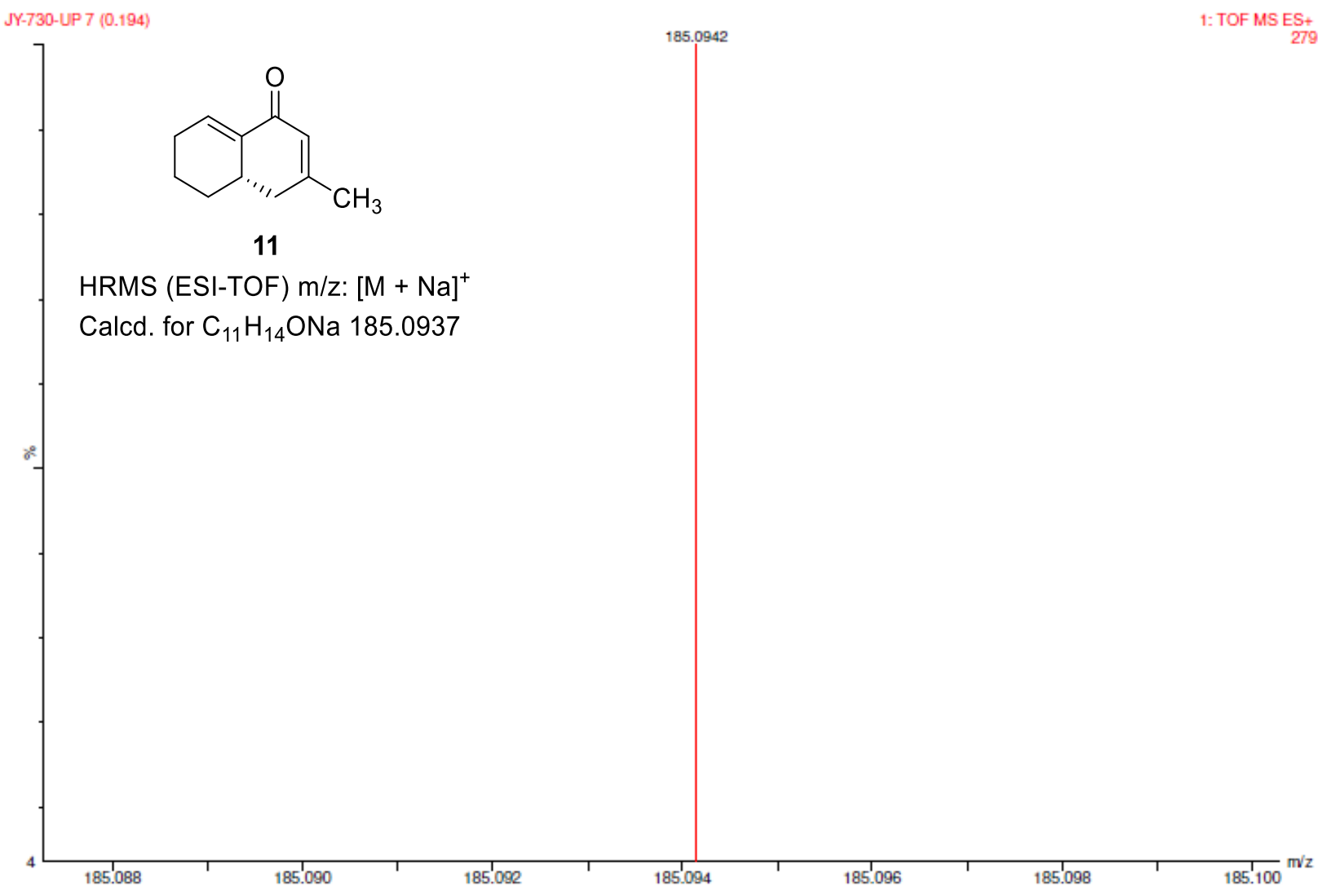




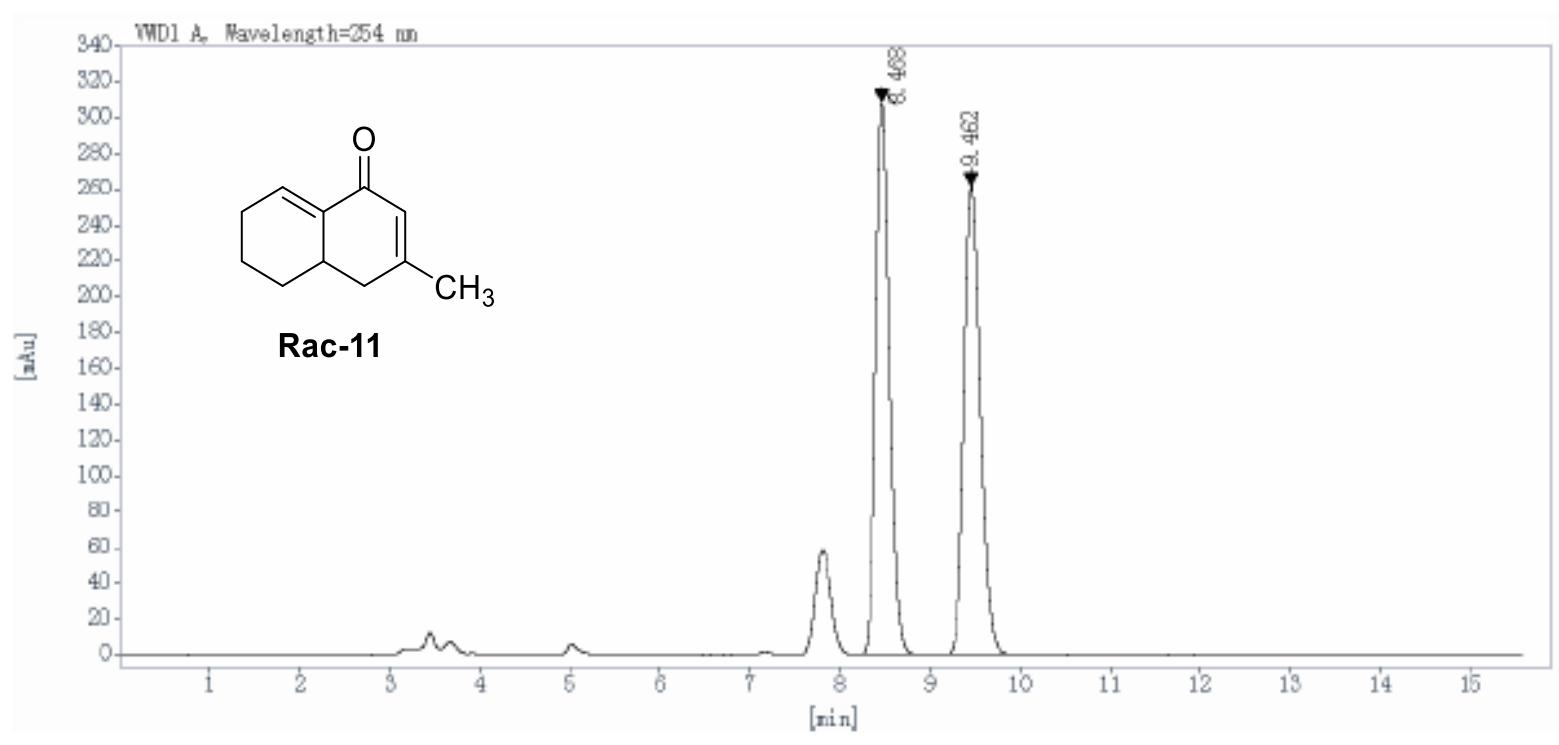

\begin{tabular}{cccccc}
$\begin{array}{c}\text { Ret Time } \\
\text { [min] }\end{array}$ & $\begin{array}{c}\text { Peak } \\
\text { Type }\end{array}$ & $\begin{array}{c}\text { Width } \\
{[\mathrm{min}]}\end{array}$ & $\begin{array}{c}\text { Height } \\
{[\mathrm{mAU}]}\end{array}$ & $\begin{array}{c}\text { Area } \\
{\left[\mathrm{mAU} \mathbf{A}^{*} \text { ] }\right]}\end{array}$ & $\begin{array}{c}\text { Area } \\
{[\%]}\end{array}$ \\
\hline 8.468 & BB & 0.16 & 309.2486 & 3261.6016 & 50.0074 \\
9.462 & BB & 0.19 & $\begin{array}{c}262.0694 \\
\text { Totals: }\end{array}$ & 3260.6313 & 49.9926 \\
& & & 652.2329 & 100.0000
\end{tabular}

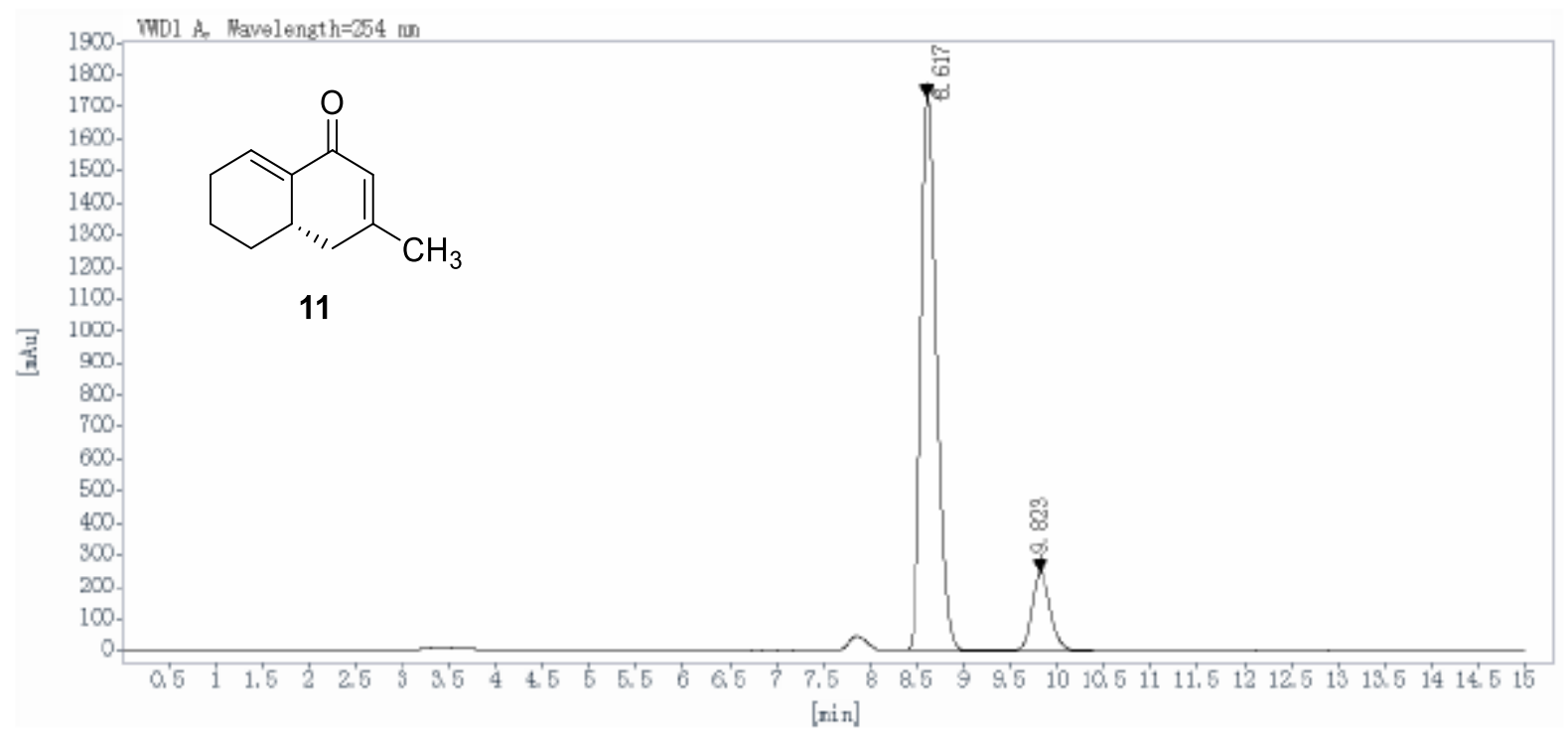

\begin{tabular}{cccccc}
$\begin{array}{c}\text { Ret Time } \\
\text { [min] }\end{array}$ & $\begin{array}{c}\text { Peak } \\
\text { Type }\end{array}$ & $\begin{array}{c}\text { Width } \\
{[\mathrm{min}]}\end{array}$ & $\begin{array}{c}\text { Height } \\
{[\mathrm{mAU}]}\end{array}$ & $\begin{array}{c}\text { Area } \\
{[\mathrm{mAU} \text { *s] }}\end{array}$ & $\begin{array}{c}\text { Area } \\
{[\%]}\end{array}$ \\
\hline 8.617 & BV & 0.18 & 1734.3817 & 20103.1699 & 85.5947 \\
9.823 & VB & 0.21 & $\begin{array}{c}246.2197 \\
\text { Totals: }\end{array}$ & 23383.3008 & 14.4053 \\
& & & 2348.4707 & 100.0000
\end{tabular}


<smiles>CCOC(=O)CC1Oc2ccccc2C=C1[N+](=O)[O-]</smiles>

\section{$13 a$}

${ }^{1} \mathrm{H}$ NMR $\left(400 \mathrm{MHz}, \mathrm{CDCl}_{3}\right)$

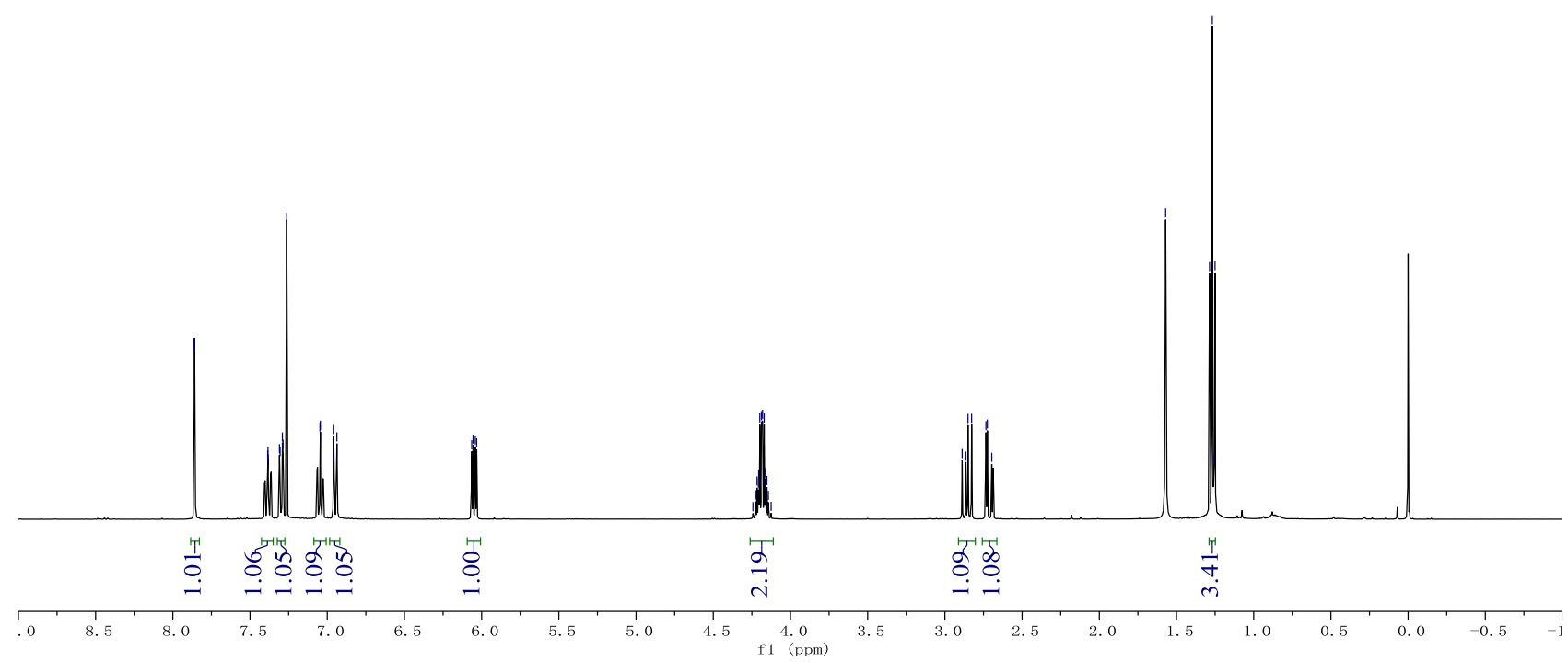

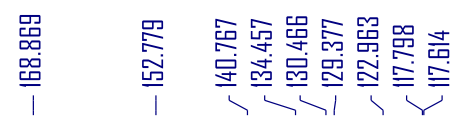

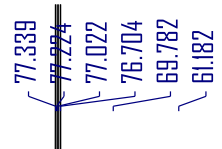

品
品<smiles>CCOC(=O)CC1Oc2ccccc2C=C1[N+](=O)[O-]</smiles>

$13 a$

${ }^{13} \mathrm{C}$ NMR $\left(100 \mathrm{MHz}, \mathrm{CDCl}_{3}\right)$

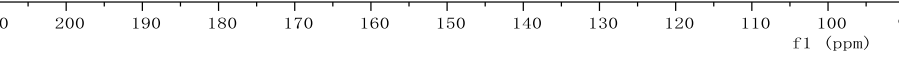




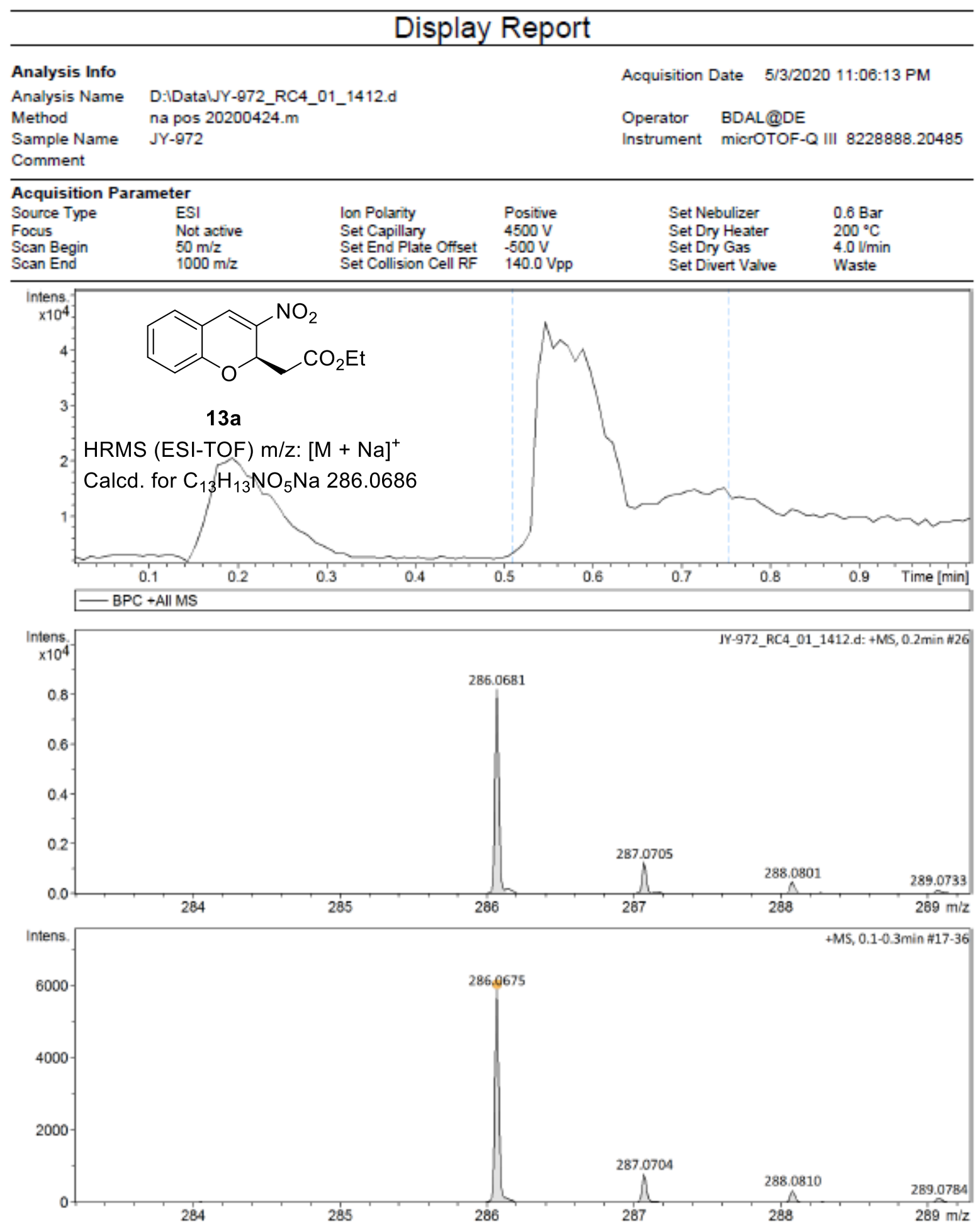




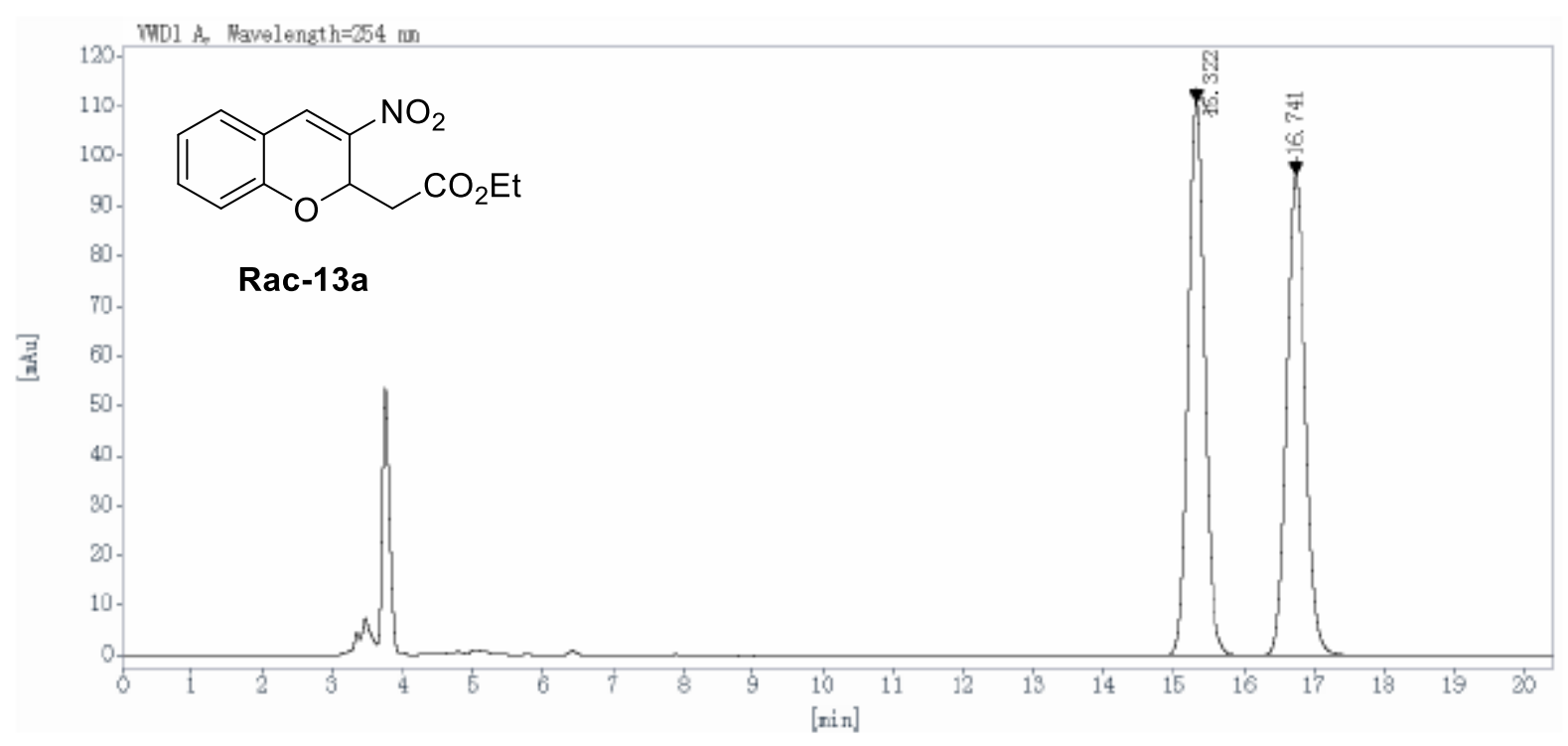

$\begin{array}{cccccc}\begin{array}{c}\text { Ret Time } \\ \text { [min] }\end{array} & \begin{array}{c}\text { Peak } \\ \text { Type }\end{array} & \begin{array}{c}\text { Width } \\ \text { [min] }\end{array} & \begin{array}{c}\text { Height } \\ \text { [mAU] }\end{array} & \begin{array}{c}\text { Area } \\ {[\mathrm{mAU} * \text { s] }}\end{array} & \begin{array}{c}\text { Area } \\ {[\%]}\end{array} \\ 15.322 & \text { BB } & 0.25 & 111.1238 & 1791.2875 & 49.9798 \\ 16.741 & \text { BB } & 0.29 & 96.4081 & 1792.7361 & 50.0202 \\ & & & \text { Totals: } & 3584.0236 & 100.0000\end{array}$

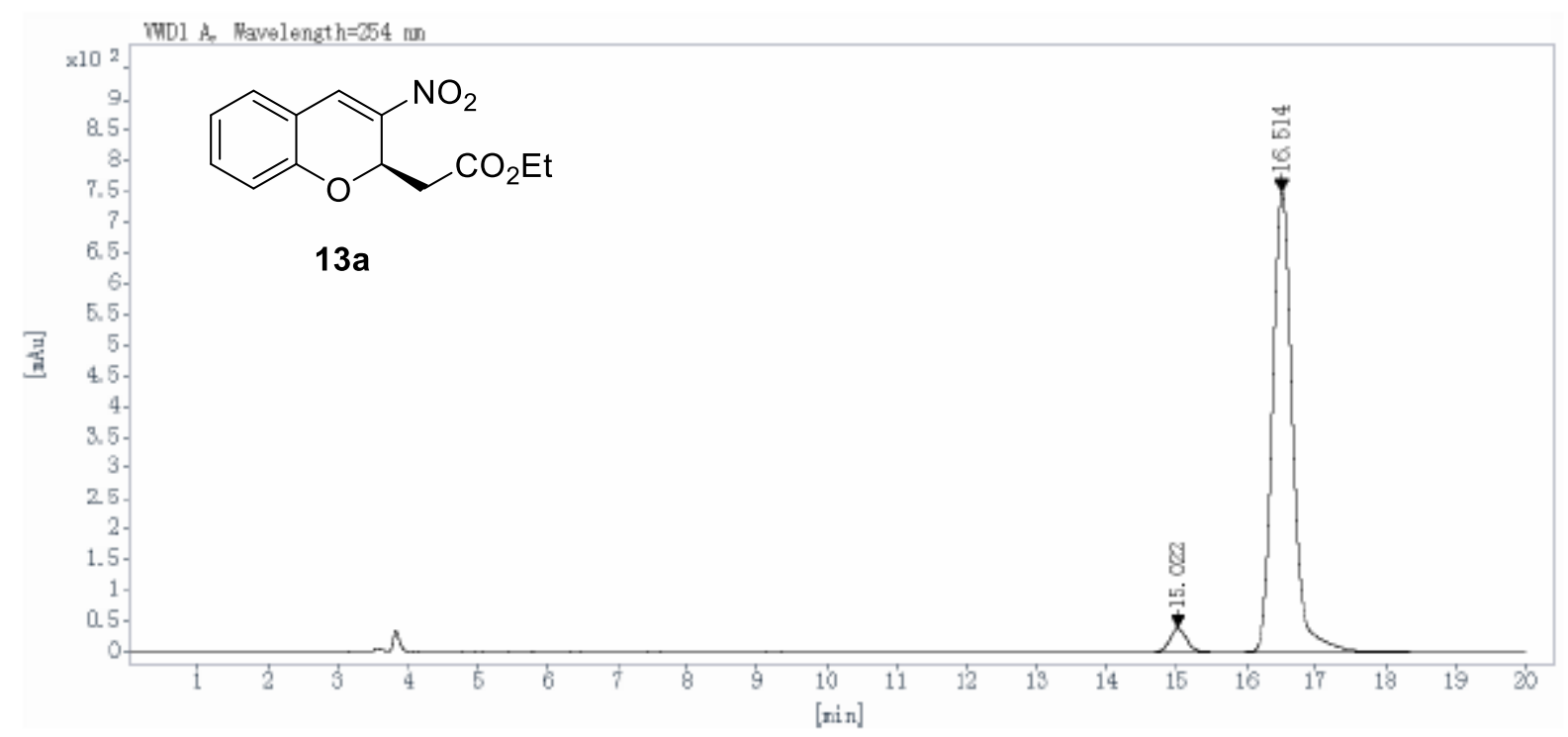

$\begin{array}{cccccc}\begin{array}{c}\text { Ret Time } \\ \text { [min] }\end{array} & \begin{array}{c}\text { Peak } \\ \text { Type }\end{array} & \begin{array}{c}\text { Width } \\ {[\mathrm{min}]}\end{array} & \begin{array}{c}\text { Height } \\ {[\mathrm{mAU}]}\end{array} & \begin{array}{c}\text { Area } \\ {[\mathrm{mAU} * \text { s] }}\end{array} & \begin{array}{c}\text { Area } \\ {[\%]}\end{array} \\ 15.022 & \text { BB } & 0.26 & 39.6913 & 656.5145 & 4.2864 \\ 16.514 & \text { BB } & 0.30 & 749.6428 & 14659.6035 & 95.7136 \\ & & & \text { Totals: } & 15316.1180 & 100.0000\end{array}$


<smiles>CC(=O)CC1Oc2ccccc2C=C1[N+](=O)[O-]</smiles>

13b

${ }^{1} \mathrm{H}$ NMR $\left(400 \mathrm{MHz}, \mathrm{CDCl}_{3}\right)$

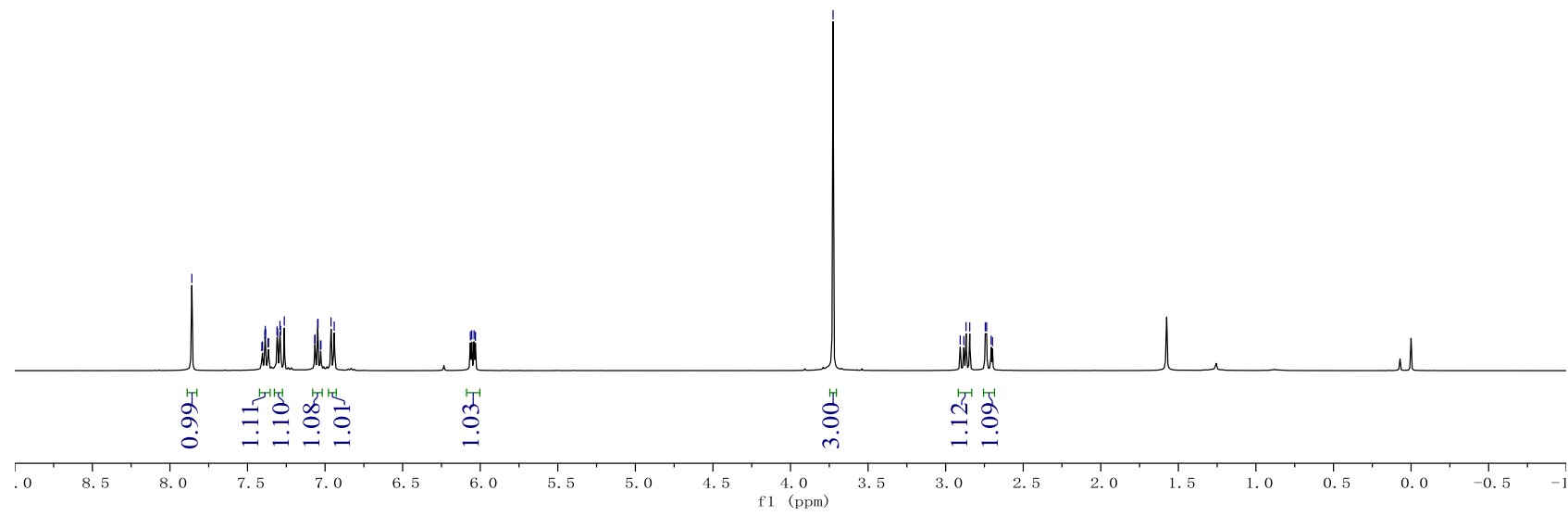

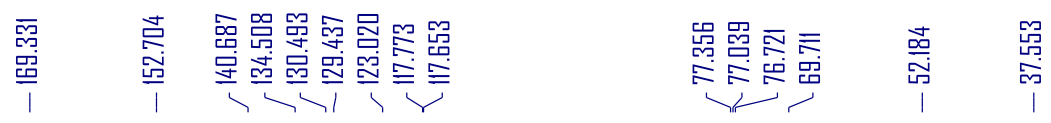<smiles>CC(=O)C[C@H]1Oc2ccccc2C=C1[N+](=O)[O-]</smiles>

13b

${ }^{13} \mathrm{C}$ NMR $\left(100 \mathrm{MHz}, \mathrm{CDCl}_{3}\right)$

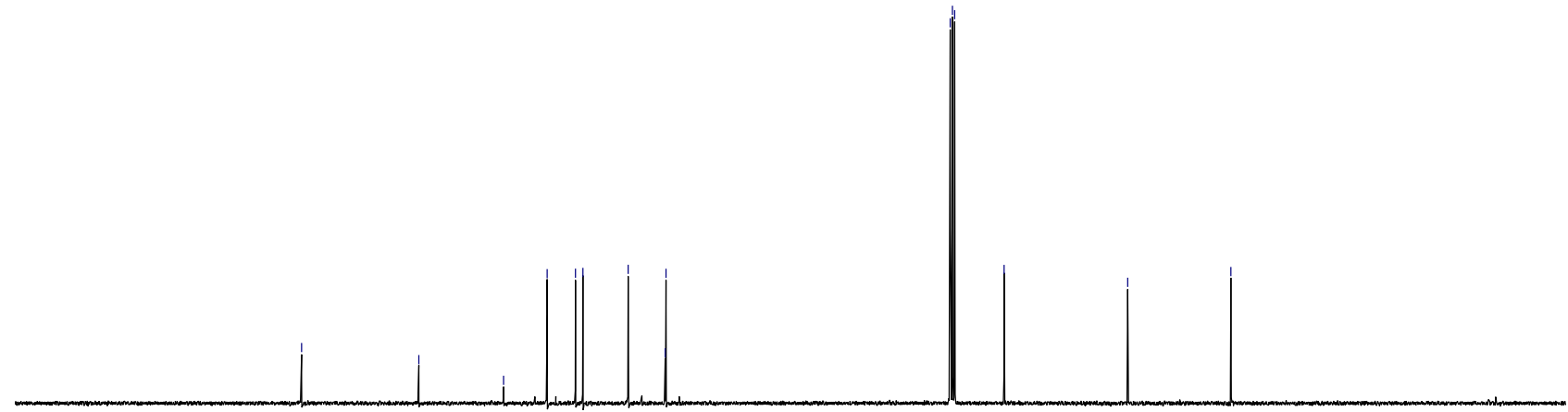

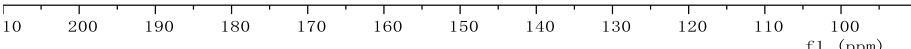



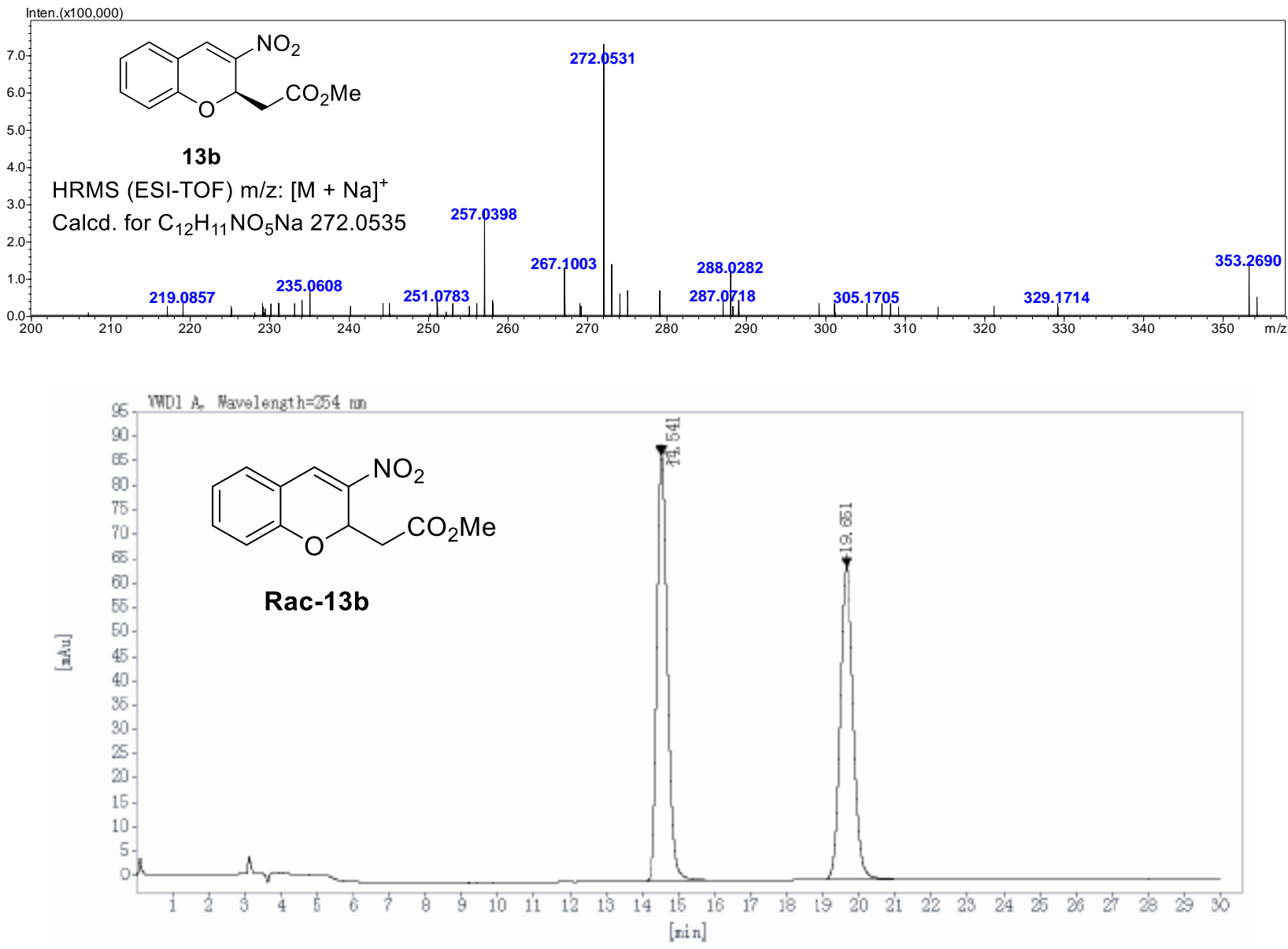

$\begin{array}{cccccc}\begin{array}{c}\text { Ret Time } \\ \text { [min] }\end{array} & \begin{array}{c}\text { Peak } \\ \text { Type }\end{array} & \begin{array}{c}\text { Width } \\ {[\mathrm{min}]}\end{array} & \begin{array}{c}\text { Height } \\ {[\mathrm{mAU}]}\end{array} & \begin{array}{c}\text { Area } \\ {\left[\mathbf{m A U} \dot{*}_{\text {s] }}\right.}\end{array} & \begin{array}{c}\text { Area } \\ {[\%]}\end{array} \\ 14.541 & \text { BB } & 0.30 & 87.3731 & 1719.8230 & 51.2896 \\ 19.651 & \text { BB } & 0.40 & 64.0335 & 1633.3365 & 48.7104 \\ & & & \text { Totals: } & 3353.1595 & 100.0000\end{array}$

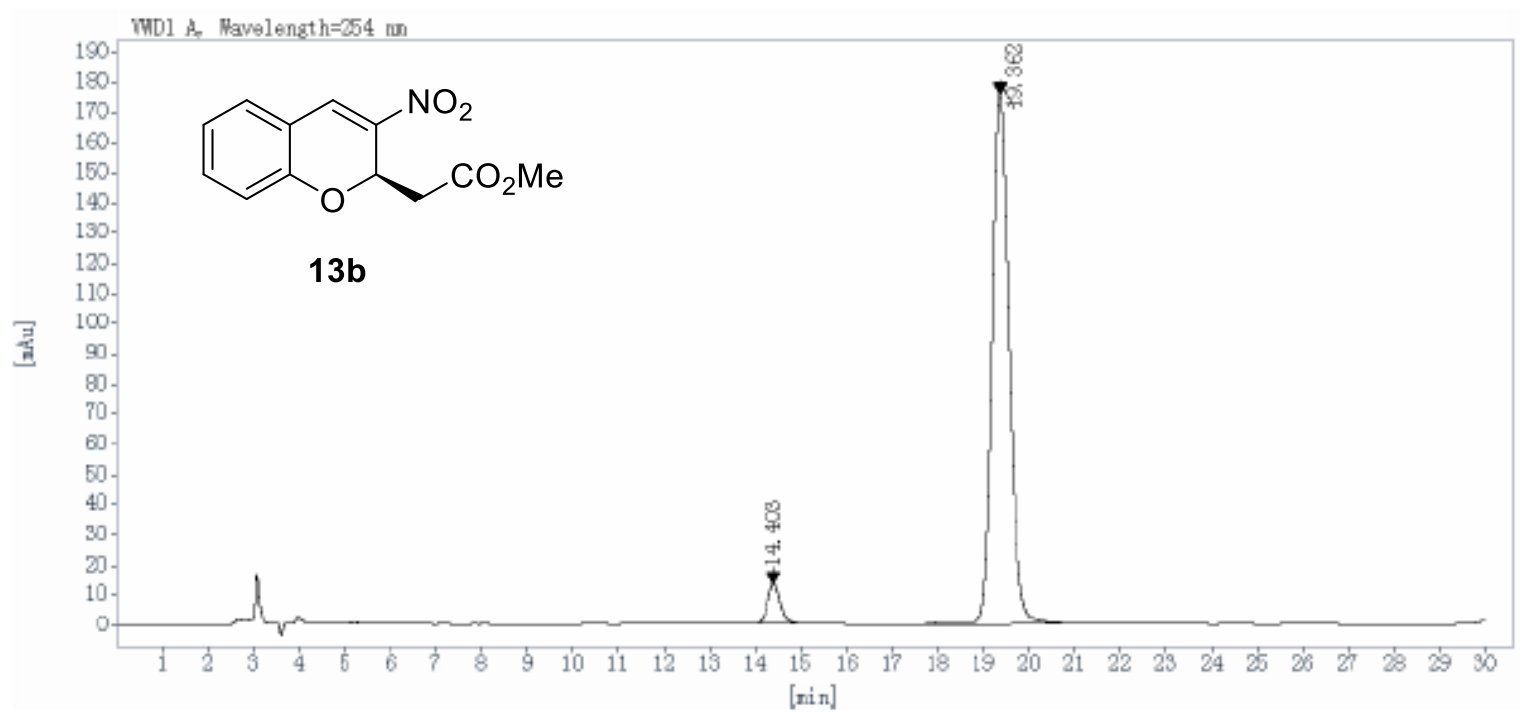

\begin{tabular}{cccccc}
$\begin{array}{c}\text { Ret Time } \\
\text { [min] }\end{array}$ & $\begin{array}{c}\text { Peak } \\
\text { Type }\end{array}$ & $\begin{array}{c}\text { Width } \\
{[\mathrm{min}]}\end{array}$ & $\begin{array}{c}\text { Height } \\
{[\mathrm{mAU}]}\end{array}$ & $\begin{array}{c}\text { Area } \\
{\left[\mathrm{mAU} \dot{*}_{\text {s }}\right]}\end{array}$ & $\begin{array}{c}\text { Area } \\
{[\%]}\end{array}$ \\
\hline 14.403 & BB & 0.30 & 13.1247 & 257.4270 & 5.3676 \\
19.362 & BB & 0.40 & $\begin{array}{c}175.6490 \\
\text { Totals: }\end{array}$ & 4538.4756 & 94.6324 \\
& & & 479.9026 & 100.0000
\end{tabular}


<smiles>COc1cccc(C(=O)C[C@H]2CCC[C@H]3c4ccc(OC)cc4C(=O)[C@H]23)c1</smiles>

${ }^{1} \mathrm{H}$ NMR $\left(400 \mathrm{MHz}, \mathrm{CDCl}_{3}\right)$

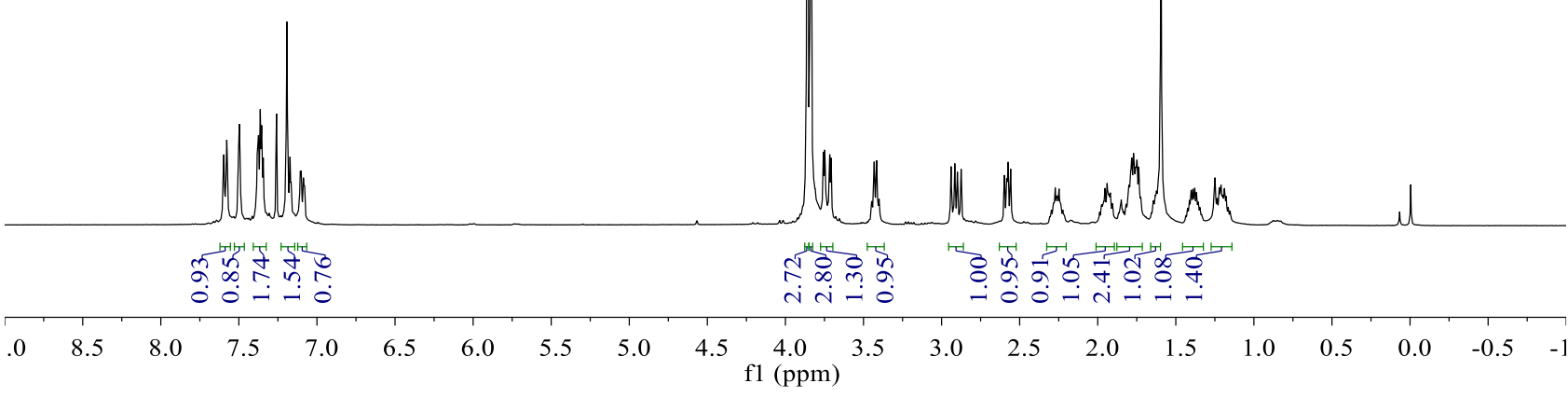

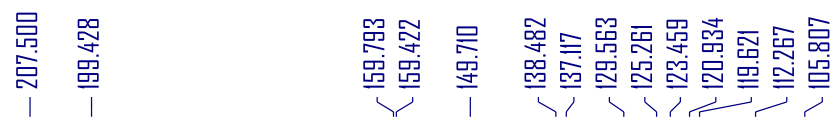<smiles>COc1cccc(C(=O)C[C@H]2CCC[C@H]3c4ccc(OC)cc4C(=O)[C@H]23)c1</smiles>

${ }^{13} \mathrm{C}$ NMR $\left(100 \mathrm{MHz}, \mathrm{CDCl}_{3}\right)$

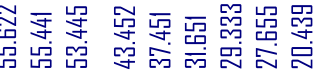

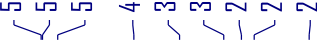

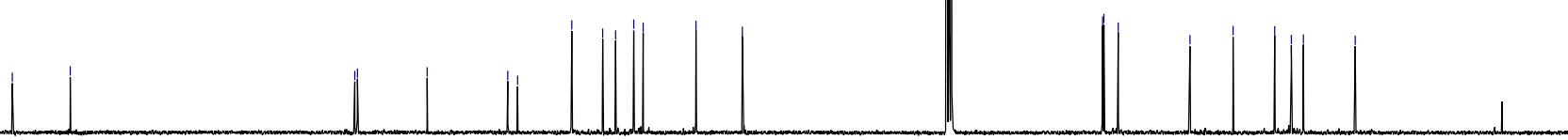

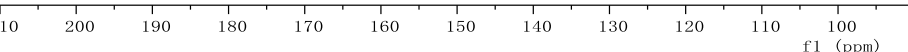



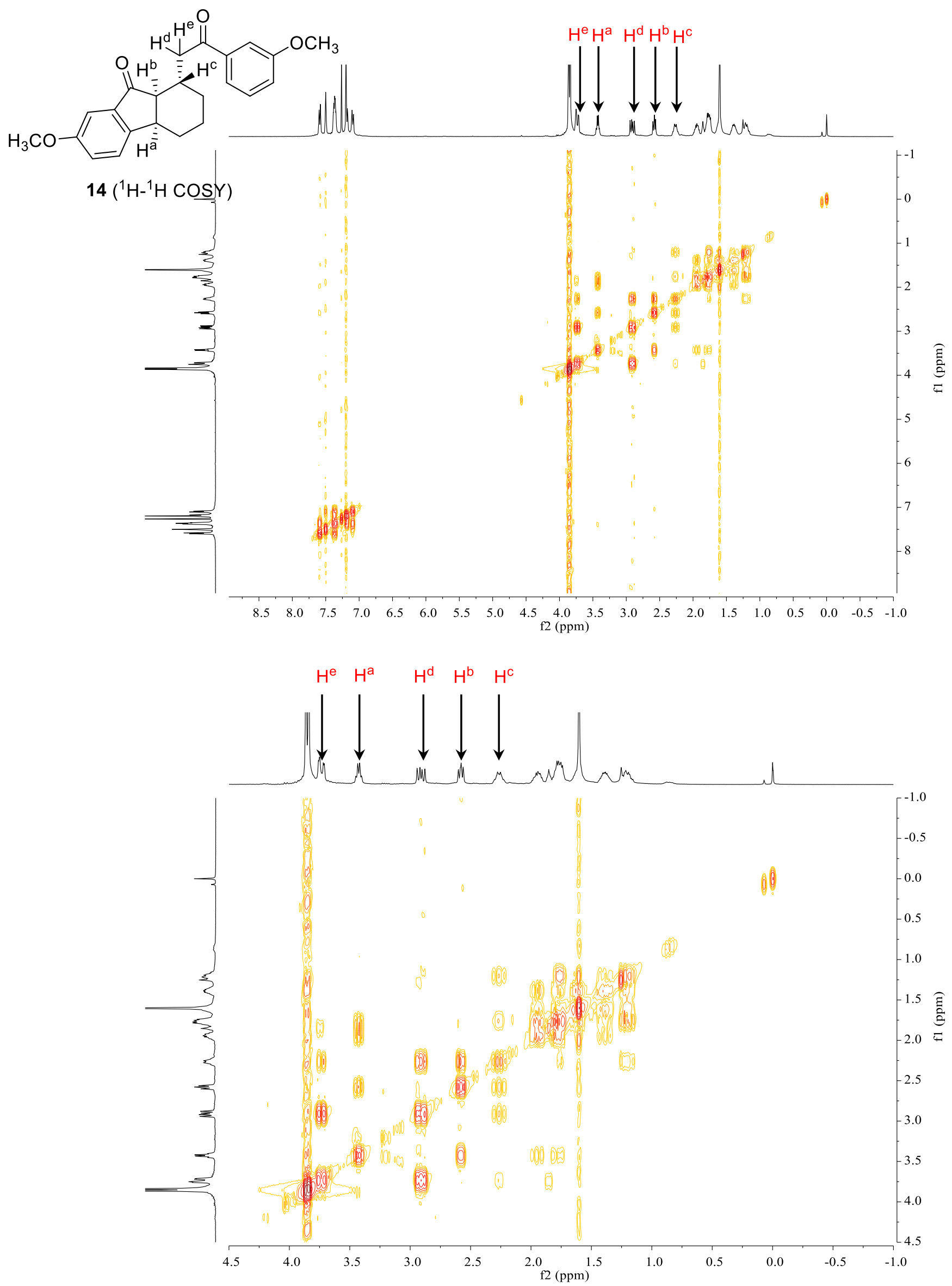
<smiles>[2H][C@@]1(CC(=O)c2cccc(OC)c2)CCC[C@H]2[C]1C(=O)c1cc(OC)ccc12</smiles>

14, NOEDS, 2.26 ppm

NOEDS NMR $\left(600 \mathrm{MHz}, \mathrm{CDCl}_{3}\right)$

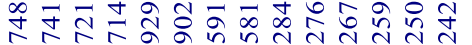

mंm

$\rightarrow$ s

no $\mathrm{H}^{\mathrm{a}}$ signal at $3.42 \mathrm{ppm}$ irradiation $\mathrm{H}^{\mathrm{c}}$ at $2.26 \mathrm{ppm}$

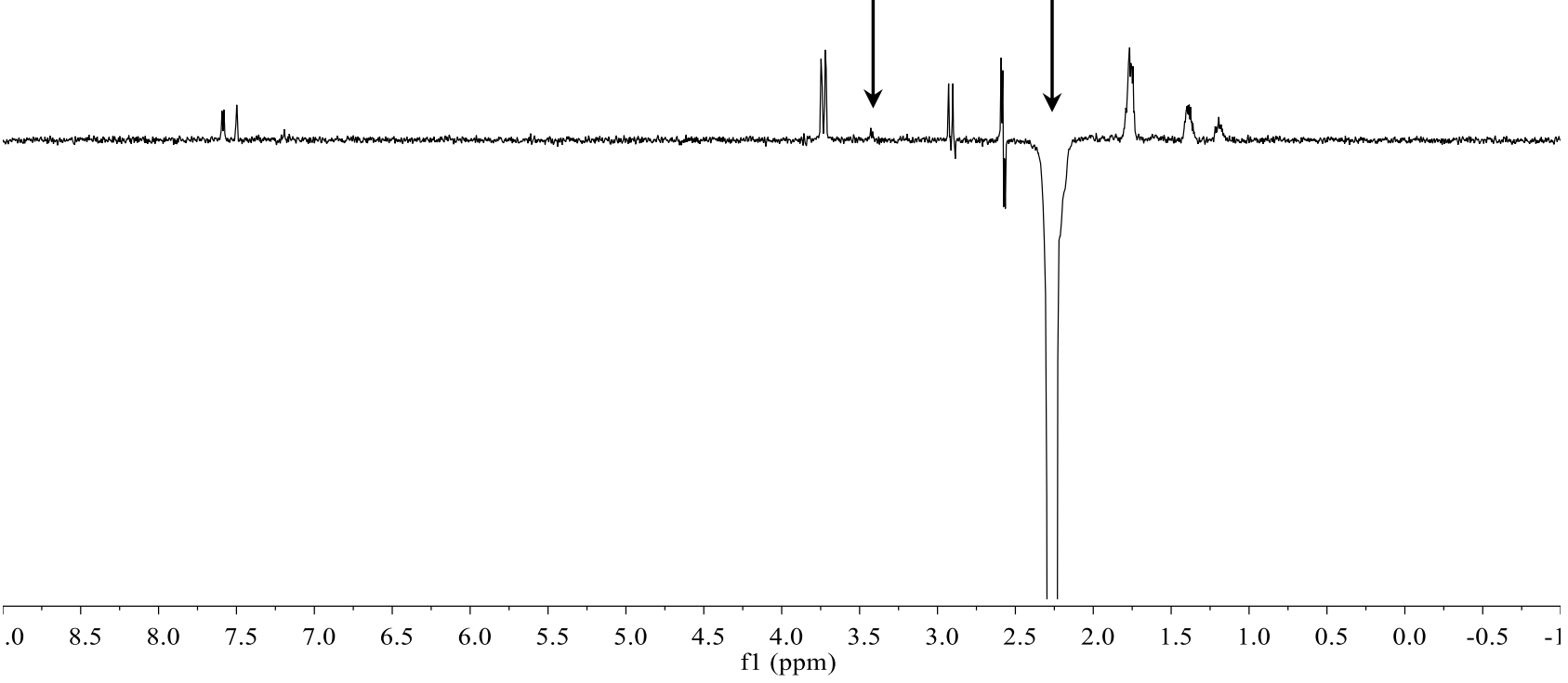<smiles>COc1cccc(C(=O)C[C@H]2CCC[C@@H]3c4ccc(OC)cc4C(=O)[C@@H]32)c1</smiles>

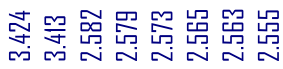

$\mathrm{H}^{\mathrm{a}}$ signal at $3.42 \mathrm{ppm} \quad$ irradiation $\mathrm{H}^{\mathrm{b}}$ at $2.58 \mathrm{ppm}$

14, NOEDS, 2.58 ppm

NOEDS NMR $\left(600 \mathrm{MHz}, \mathrm{CDCl}_{3}\right)$
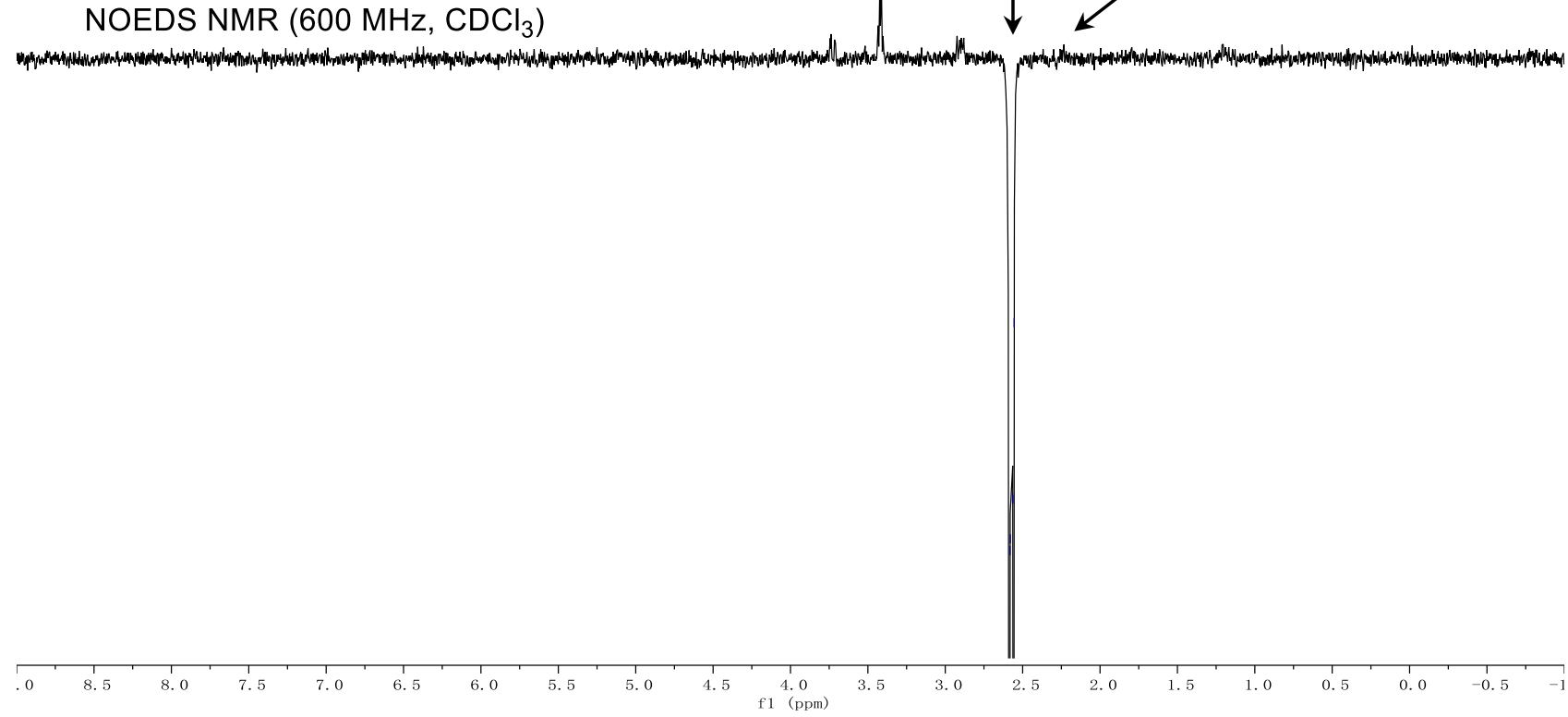


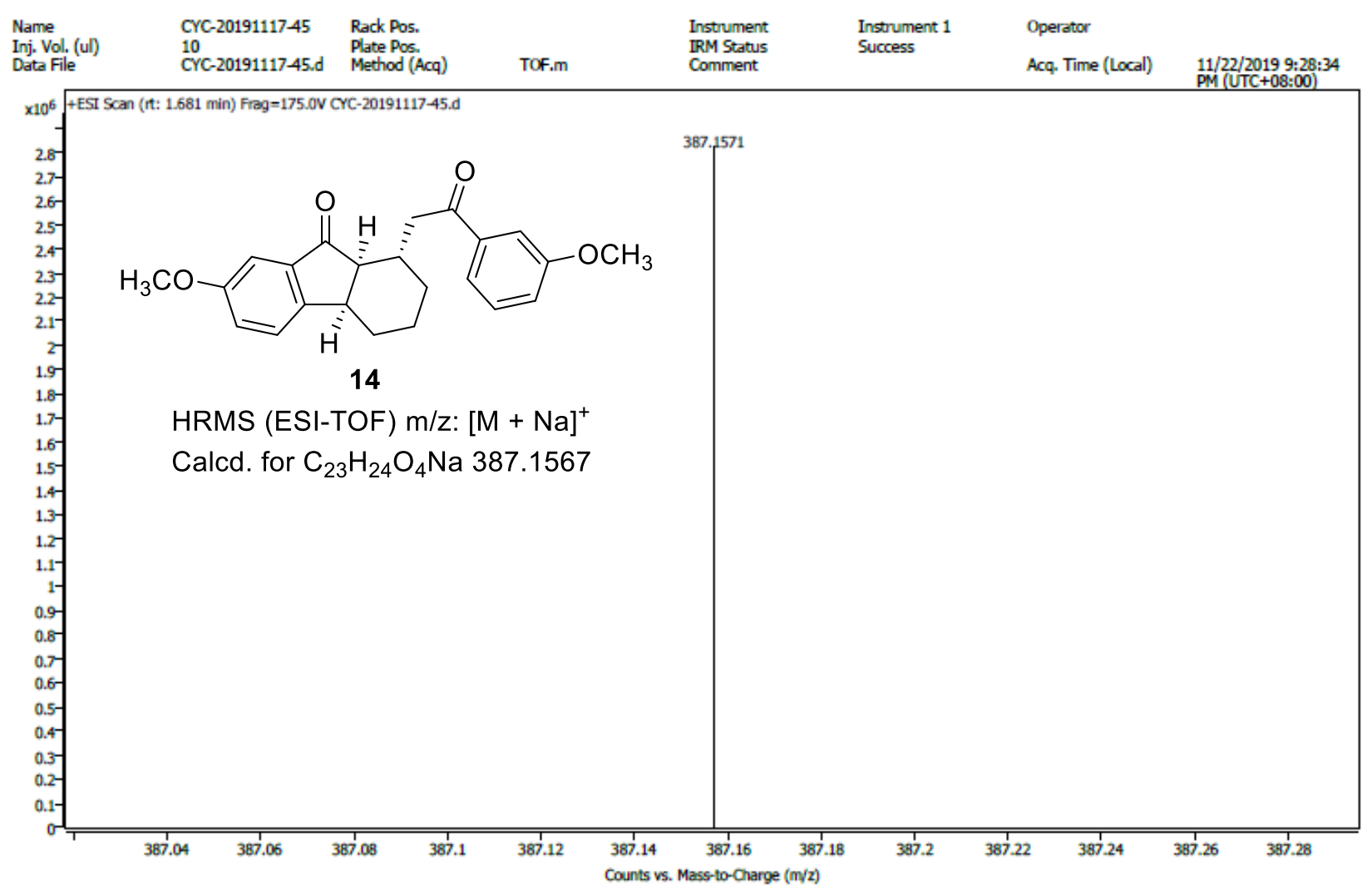




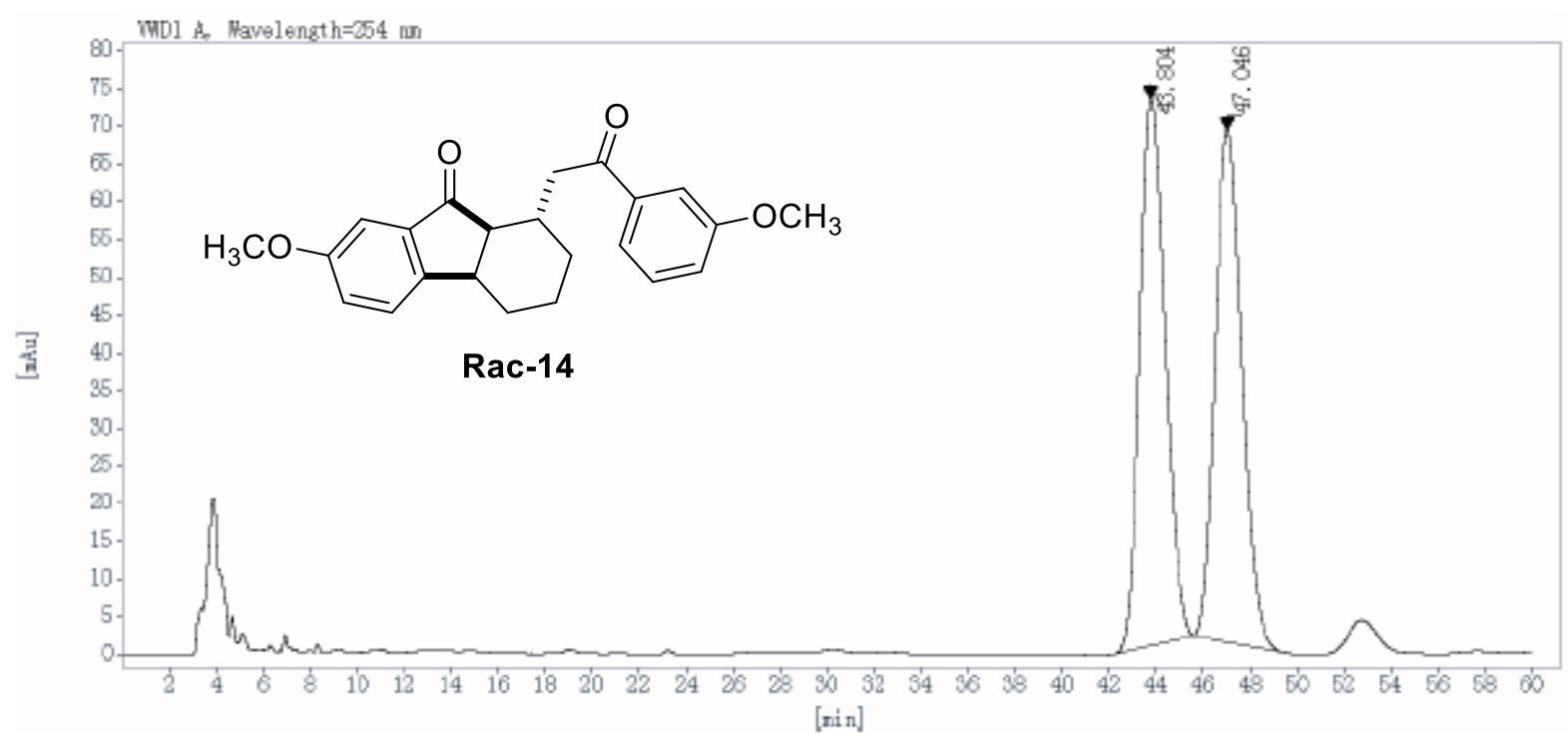

\begin{tabular}{cccccc}
$\begin{array}{c}\text { Ret Time } \\
\text { [min] }\end{array}$ & $\begin{array}{c}\text { Peak } \\
\text { Type }\end{array}$ & $\begin{array}{c}\text { Width } \\
\text { [min] }\end{array}$ & $\begin{array}{c}\text { Height } \\
{[\mathrm{mAU} \text { ] }}\end{array}$ & $\begin{array}{c}\text { Area } \\
{[\mathrm{mAU} \text { *s] }}\end{array}$ & $\begin{array}{c}\text { Area } \\
{[\%]}\end{array}$ \\
\hline 43.804 & BB & 1.16 & 72.5445 & 5438.1040 & 50.1553 \\
47.046 & BB & 1.23 & 67.9043 & 5404.4326 & 49.8447 \\
& & & Totals: & 10842.5366 & 100.0000
\end{tabular}

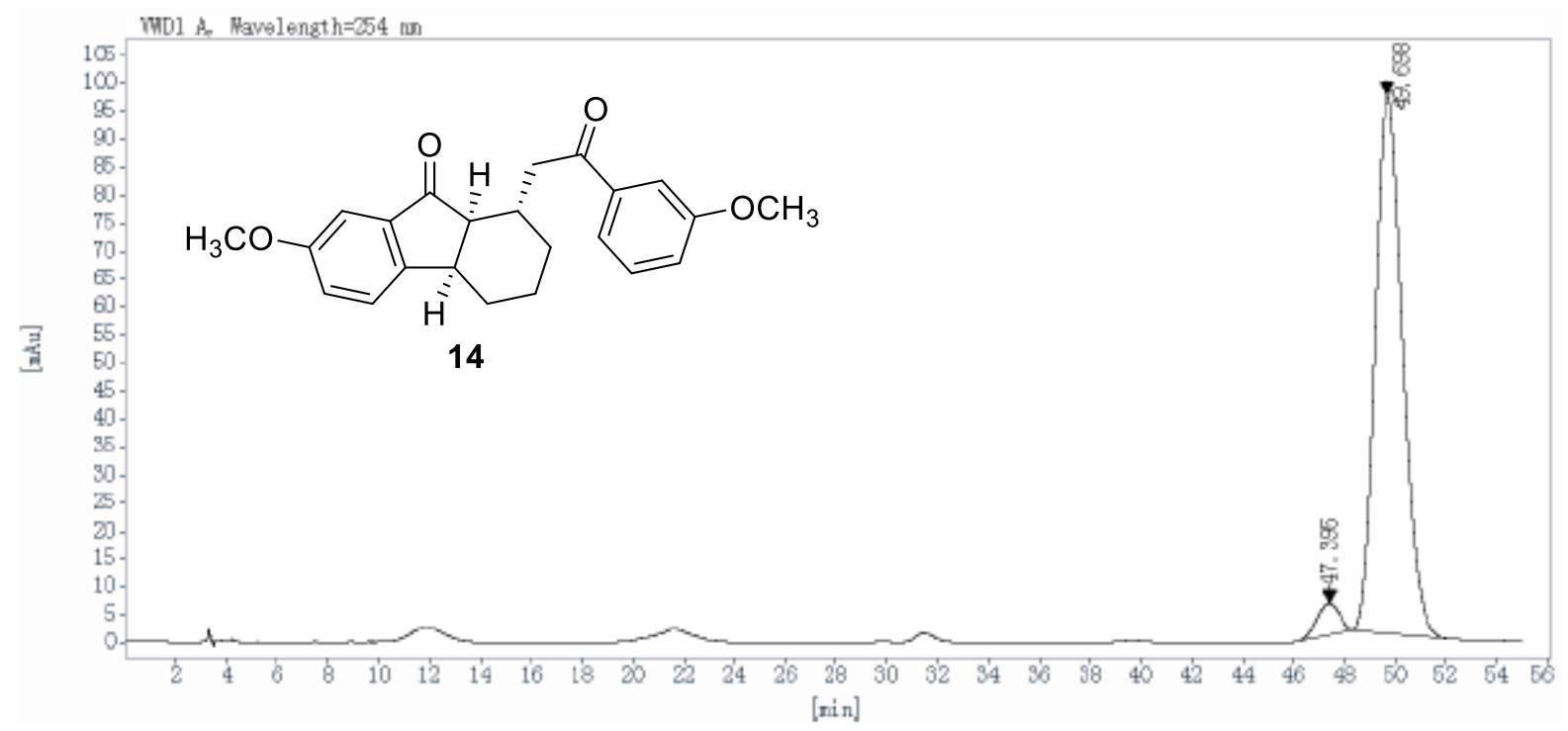

\begin{tabular}{cccccc}
$\begin{array}{c}\text { Ret Time } \\
\text { [min] }\end{array}$ & $\begin{array}{c}\text { Peak } \\
\text { Type }\end{array}$ & $\begin{array}{c}\text { Width } \\
\text { [min] }\end{array}$ & $\begin{array}{c}\text { Height } \\
{[\mathrm{mAU}]}\end{array}$ & $\begin{array}{c}\text { Area } \\
{\left[\mathrm{mAU} \mathbf{A U}^{*} \text { ] }\right.}\end{array}$ & $\begin{array}{c}\text { Area } \\
{[\%]}\end{array}$ \\
\hline 47.395 & BB & 0.92 & 5.6402 & 339.7094 & 4.4690 \\
49.698 & BBA & 1.17 & 96.3931 & 7261.8213 & 95.5310 \\
& & & Totals: & 7601.5306 & 100.0000
\end{tabular}


<smiles>O=C(C[C@@H]1C[C@@H](c2ccc(Cl)cc2)C[C@H]2c3c(ccc4ccccc34)C(=O)[C@H]12)c1ccc2ccccc2c1</smiles>

15

${ }^{1} \mathrm{H}$ NMR $\left(400 \mathrm{MHz}, \mathrm{CDCl}_{3}\right)$

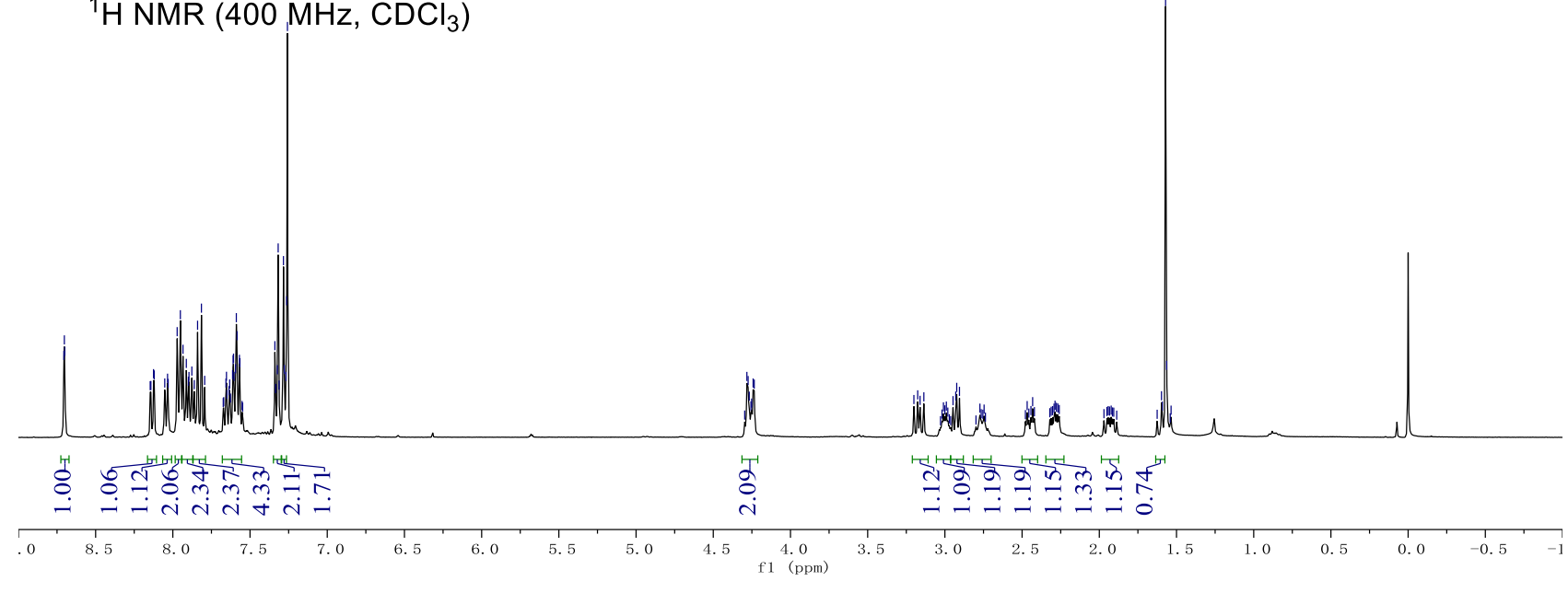

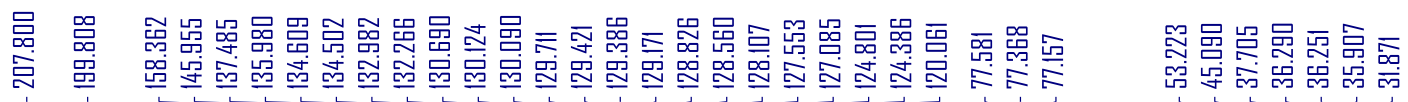<smiles>O=C(C[C@@H]1C[C@@H](c2ccc(Cl)cc2)C[C@H]2c3c(ccc4ccccc34)C(=O)[C@H]12)c1ccc2ccccc2c1</smiles>

15

${ }^{13} \mathrm{C}$ NMR $\left(150 \mathrm{MHz}, \mathrm{CDCl}_{3}\right)$

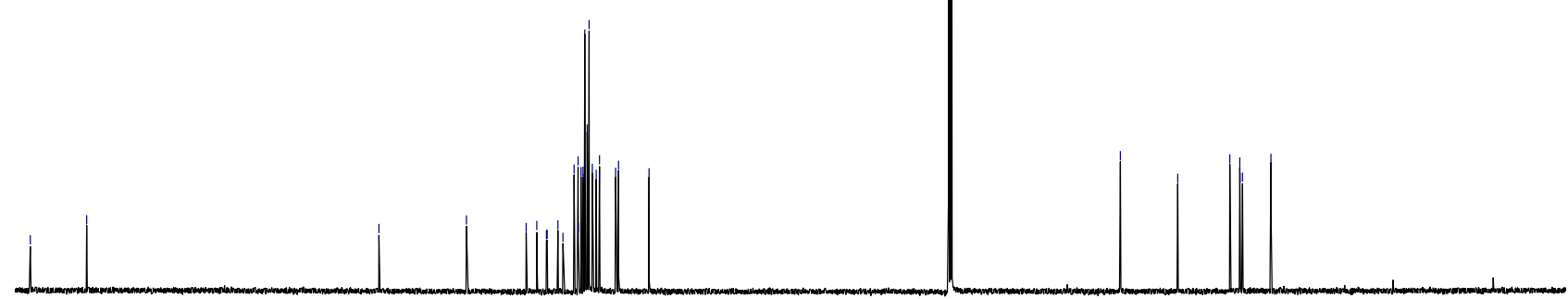




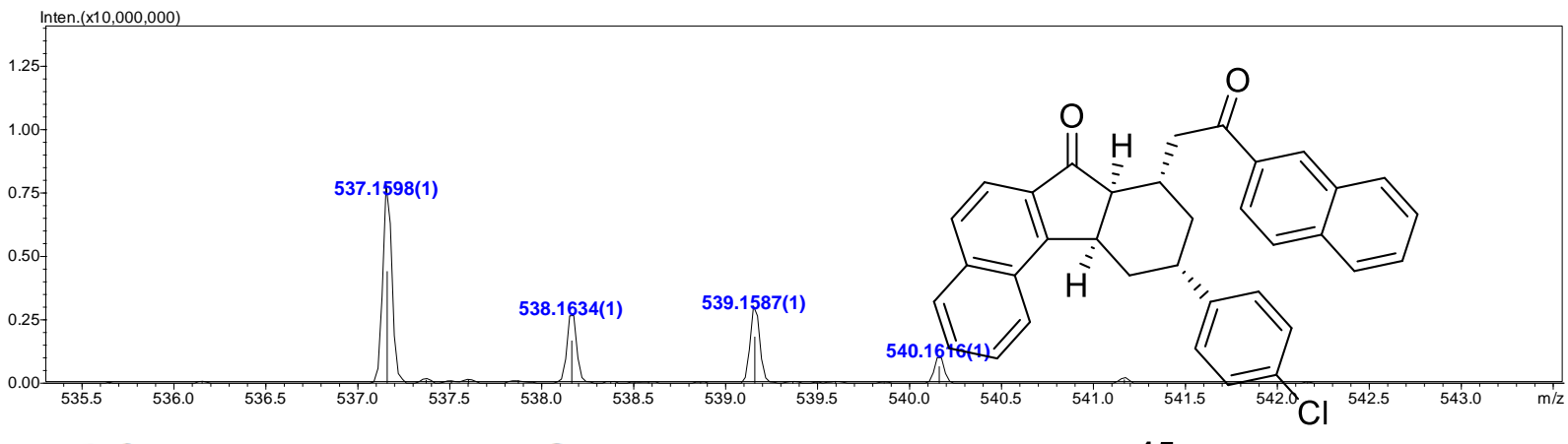

15

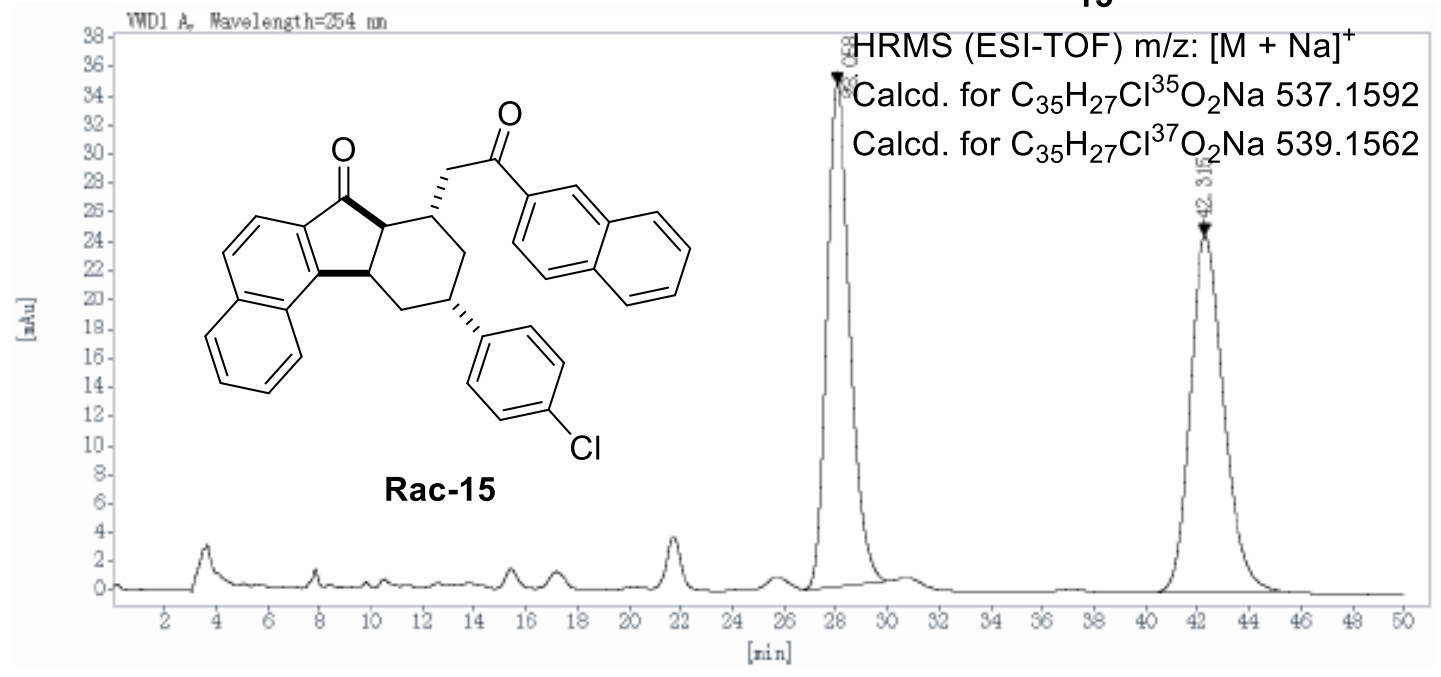

$\begin{array}{cccccc}\begin{array}{c}\text { Ret Time } \\ \text { [min] }\end{array} & \begin{array}{c}\text { Peak } \\ \text { Type }\end{array} & \begin{array}{c}\text { Width } \\ {[\mathrm{min}]}\end{array} & \begin{array}{c}\text { Height } \\ {[\mathrm{mAU}]}\end{array} & \begin{array}{c}\text { Area } \\ {\left[\mathrm{mAU} \mathbf{*}_{\text {s] }}\right]}\end{array} & \begin{array}{c}\text { Area } \\ {[\%]}\end{array} \\ 28.058 & \text { BB } & 0.89 & 34.5757 & 2159.5308 & 49.4736 \\ 42.315 & \text { BBA } & 1.36 & 24.5106 & 2205.4873 & 50.5264 \\ & & & \text { Totals: } & 4365.0181 & 100.0000\end{array}$

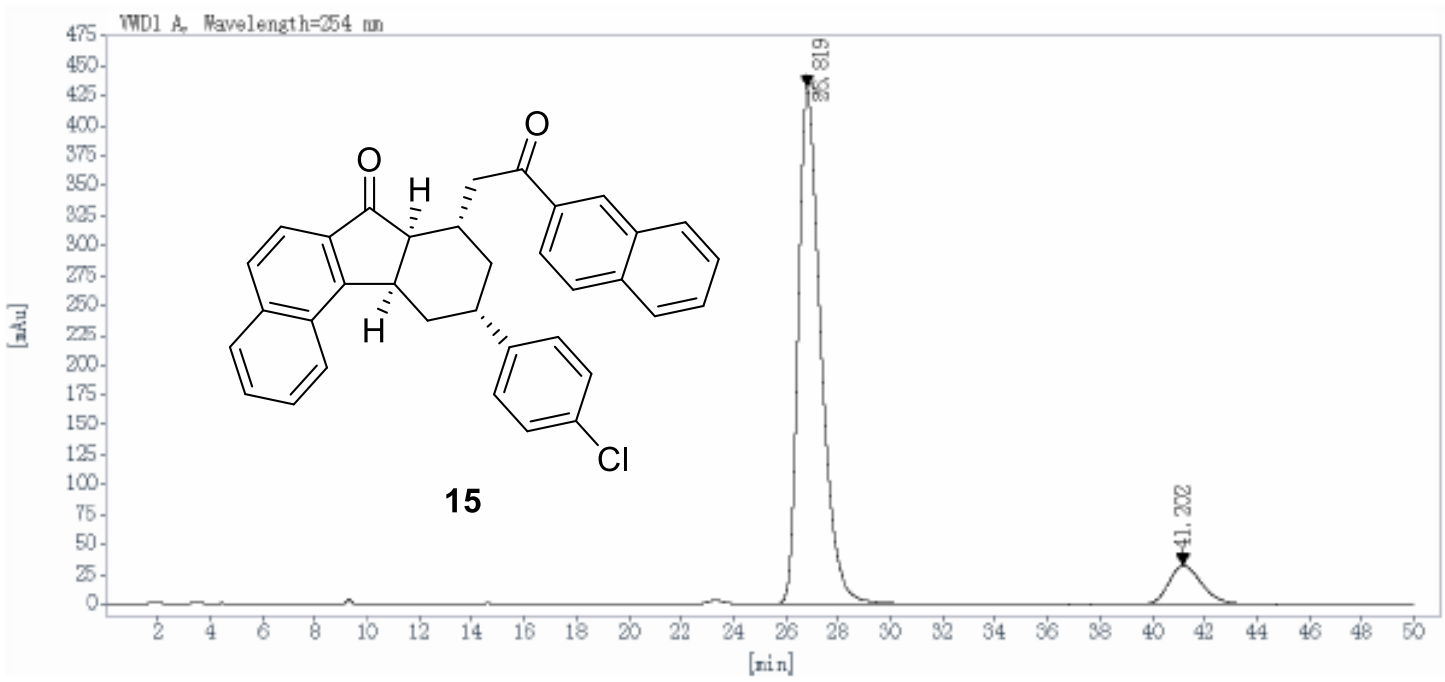

$\begin{array}{cccccc}\begin{array}{c}\text { Ret Time } \\ \text { [min] }\end{array} & \begin{array}{c}\text { Peak } \\ \text { Type }\end{array} & \begin{array}{c}\text { Width } \\ {[\mathrm{min}]}\end{array} & \begin{array}{c}\text { Height } \\ {[\mathrm{mAU}]}\end{array} & \begin{array}{c}\text { Area } \\ {[\mathrm{mAU} * \mathrm{~s}]}\end{array} & \begin{array}{c}\text { Area } \\ {[\%]}\end{array} \\ 26.819 & \text { BBA } & 0.93 & 432.5074 & 26309.1484 & 90.4041 \\ 41.202 & \text { BBA } & 1.30 & 32.4633 & 2792.5610 & 9.5959 \\ & & & \text { Totals: } & 29101.7095 & 100.0000\end{array}$

